LA W RENCE LIVERMORE N A TIONAL LABORATORY
Specular Reflectivity and Hot-Electron Generation in High-Contrast Relativistic Laser-Plasma Interactions

G. E. Kemp

July 9, 2013 
This document was prepared as an account of work sponsored by an agency of the United States government. Neither the United States government nor Lawrence Livermore National Security, LLC, nor any of their employees makes any warranty, expressed or implied, or assumes any legal liability or responsibility for the accuracy, completeness, or usefulness of any information, apparatus, product, or process disclosed, or represents that its use would not infringe privately owned rights. Reference herein to any specific commercial product, process, or service by trade name, trademark, manufacturer, or otherwise does not necessarily constitute or imply its endorsement, recommendation, or favoring by the United States government or Lawrence Livermore National Security, LLC. The views and opinions of authors expressed herein do not necessarily state or reflect those of the United States government or Lawrence Livermore National Security, LLC, and shall not be used for advertising or product endorsement purposes.

This work performed under the auspices of the U.S. Department of Energy by Lawrence Livermore National Laboratory under Contract DE-AC52-07NA27344. 


\title{
SPECULAR REFLECTIVITY AND Hot-Electron Generation in High-Contrast Relativistic LASER-PLASMA INTERACTIONS
}

\author{
DISSERTATION
}

Presented in Partial Fulfillment of the Requirements for the Degree Doctor of Philosophy in the Graduate School of The Ohio State University

By

Gregory Elijah Kemp, B.S., M.S.

Graduate Program in Physics

The Ohio State University

2013

Dissertation Committee:

Distinguished Professor Richard R. Freeman, Advisor

Associate Provost \& Director, Professor Linn D. Van Woerkom

Professor Richard Furnstahl

Professor Richard Hughes 
(C) Copyright by

Gregory Elijah Kemp

2013 


\section{ABSTRACT}

Ultra-intense laser $\left(>10^{18} \mathrm{~W} / \mathrm{cm}^{2}\right)$ interactions with matter are capable of producing relativistic electrons which have a variety of applications in state-of-the-art scientific and medical research conducted at universities and national laboratories across the world. Control of various aspects of these hot-electron distributions is highly desired to optimize a particular outcome. Hot-electron generation in low-contrast interactions, where significant amounts of under-dense pre-plasma are present, can be plagued by highly non-linear relativistic laser-plasma instabilities and quasi-static magnetic field generation, often resulting in less than desirable and predictable electron source characteristics. High-contrast interactions offer more controlled interactions but often at the cost of overall lower coupling and increased sensitivity to initial target conditions. An experiment studying the differences in hot-electron generation between high and low-contrast pulse interactions with solid density targets was performed on the Titan laser platform at the Jupiter Laser Facility at Lawrence Livermore National Laboratory in Livermore, CA. To date, these hot-electrons generated in the laboratory are not directly observable at the source of the interaction. Instead, indirect studies are performed using state-of-the-art simulations, constrained by the various experimental measurements. These measurements, more-often-than-not, rely on secondary processes generated by the transport of these electrons through the solid density materials which can susceptible to a variety instabilities and target material/geometry effects. Although often neglected in these types of studies, the specularly reflected light can provide invaluable insight as it is directly influenced by the interaction.

In this thesis, I address the use of (personally obtained) experimental specular reflectivity measurements to indirectly study hot-electron generation in the context of high-contrast, 
relativistic laser-plasma interactions. Spatial, temporal and spectral properties of the incident and specular pulses, both near and far away from the interaction region where experimental measurements are obtained, are used to benchmark simulations designed to infer dominant hot-electron acceleration mechanisms and their corresponding energy/angular distributions. To handle this highly coupled interaction, I employed particle-in-cell modeling using a wide variety of algorithms (verified to be numerically stable and consistent with analytic expressions) and physical models (validated by experimental results) to reasonably model the interaction's sweeping range of plasma densities, temporal and spatial scales, electromagnetic wave propagation and its interaction with solid density matter. Due to the fluctuations in the experimental conditions and limited computational resources, only a limited number of full-scale simulations were performed under typical experimental conditions to infer the relevant physical phenomena in the interactions. I show the usefulness of the often overlooked specular reflectivity measurements in constraining both high and low-contrast simulations, as well as limitations of their experimental interpretations. Using these experimental measurements to reasonably constrain the simulation results, I discuss the sensitivity of relativistic electron generation in ultra-intense laser plasma interactions to initial target conditions and the dynamic evolution of the interaction region.

This work was performed under DOE contract DE-AC52-07NA27344 with support from the Lawrence Scholar Program, OFES-NNSA Joint Program in High-Energy-Density Laboratory Plasmas and an allocation of computing time from the LLNL Grand Challenge and the Ohio Supercomputing Center. 
To my amazing family, beautiful wife Dana Marie and rosy-cheeked, milk-fattened baby girl Bonnie Anne. 


\section{ACKNOWLEDGMENTS}

First and foremost, I would like to express my deepest gratitude to my amazing wife Dana Marie and beautiful newborn daughter Bonnie Anne for enduring all the late nights, hard deadlines, extended experiments, travel away from home and the perpetual science-mania associated with being a physics graduate student. I would have surely gone mad by now without their unrelenting support, encouragement, and understanding nature.

Growing up in a family with 7 siblings wasn't always easy, especially being one of the older ones who are inevitably held responsible for the younger sibling's often less than desirable actions. Although you might expect less attention from the parents in such a large family, Mom and Dad have always taken the time to teach each of us about the important things in life and encourage us to actively pursue our interests (and still do, even if it means living across the country). Family has always been of upmost importance to me and correspondingly I have always been blessed with much needed advice and support from my siblings, grandparents, aunts and uncles, cousins and friends. If it wasn't for my father and grandfather bestowing their wonder of science and the self-rewarding nature of 'hands-on' projects to me, I wouldn't be where I am today.

I have been fortunate enough to have participated in many programs that have provided invaluable contacts along the way and helped me get to where I am today. With the postsecondary option at Ohio University Eastern, I was able to get an invaluable full 2-year head start on college courses while still in high school. While an undergraduate at Purdue, The Society of Physics Students introduced the means for me to express some of my engineering tendencies where I helped motorize a Duck ${ }^{\circledR}$-tape covered love-seat, affectionately dubbed 'MoJo,' which received national recognition on ABC's Good Morning America. Seriously 
though, my undergraduate research at Purdue with in-space propulsion (thanks to Professor Ivana Hrbud) opened the opportunity of a summer internship in NASA's Undergraduate Student Research Program at the Marshall Space Flight Center in Huntsville, AL where I had the pleasure of working with John Dankanich, Gordon Woodcock and Kurt Polzin.

Upon entering graduate school, I was introduced to this research group, lead by Distinguished Professor Richard R. Freeman, by Linn D. VanWoerkom. To them, I am eternally grateful for introducing me to the fascinating (although often aggravating) topic of relativistic laser-plasma interactions and giving me the opportunity to travel across the country for experimental campaigns at some of the worlds most impressive lasers facilities. Douglass W. Schumacher, although not an official advisor on paper, has always provided invaluable support and a unique perspective when direly needed; his attention to detail and ability to concisely communicate ideas still amazes me to this day. My thanks goes out to everyone I have worked with Ohio State both past and present: Kramer Akli, Enam Chowdhury, John Marketon, Mike Storm, Vladimir Ovchinnikov, John Morrison, Andy Krygier, Rebecca Daskalova, Chris Willis, Sheng Jiang, Douglass Wertepney, Frank King, Jim King, Chris Orban, Patrick Poole, Dustin Offerman, Chris Murphy and all the undergraduates whose names I have inadvertently forgotten. Above all, I thank you all for making Columbus, $\mathrm{OH}$ feel like a home-away-from-home.

Participating in the Lawrence Scholar Program (LSP) at the Lawrence Livermore National Laboratory for the past 2 years has provided some great opportunities with it's unparalleled computational and experimental facilities and in-house expertise (with a special thanks to program leaders Anna Wright and Christine Zachow). I am grateful to my LSP advisors Pavesh K. Patel and Harry McLean for their continuing advise and innate ability to help me 'not loose the forest through the trees.' An additional thanks goes out to the tremendous support from LLNL coworkers Matthew Levy, Drew Higginson, Alessio Morace, Claudio Bellei, Frédéric Pérez, Cliff Chen, Tammy Ma, Frederico Fiúza, Andreas Kemp, Andy Mackinnon, Andrew MacPhee, Hiroshi Sawada, Scott Wilks, Hui Chen and Andy Hazi. I also would like the Jupiter Laser Facility staff for all their support of our seemingly random, last minute requests, in particular Jim Bonlie, Rich Combs, Rob Costa, 
Gary Freeze, Beth Marioitti, Bob Cauble, and Steven Maricle.

A special thanks goes out to Yuan Ping of LLNL who not only inspired most of my experimental research, but also continues to advise and provide exciting new experimental opportunities. Without Tony Link (OSU/LLNL), most of this work would have fell apart at the seams. His unmatched ability to simultaneously run both the experiment and provide simulation support has proven to be an invaluable to my research, which almost makes up for having spent more time traveling to 'exotic' places and sharing a hotel room with him than my wife!

Last, but not least, are all the experimental collaborators without which much of this work would not be possible. From the University of Alberta, a special thanks to Professor Robert Fedosejevs, Henry Tiedje, Ying Tsui, Shaun Kerr, Hal Friesen and Mianzhen Mo. From UCSD, Professor Farhat Beg, Charlie Jarrott, Anna Sorokovikova, Bob Madden, Joohwan Kim, Sugreev Chawla, Brad Westover, Toshi Yabuuchi and Chris McGuffey. From the Trident Laser Facility at Los Alamos National Laboratory, Donald 'Cort' Gautier and Randy Johnson. From General Atomics, Mingsheng Wei and Rich Stephens.

This work was performed under DOE contract DE-AC52-07NA27344 with support from the Lawrence Scholar Program, OFES-NNSA Joint Program in High-Energy-Density Laboratory Plasmas and an allocation of computing time from the LLNL Grand Challenge and the Ohio Supercomputing Center. 


\section{VITA}

April $1986 \ldots \ldots \ldots \ldots \ldots \ldots \ldots \ldots \ldots \ldots$ Born, Wheeling, WV

June 2002 - June 2004 .................. Post-Secondary Enrollment Options Program, Ohio University Eastern, St. Clairsville, $\mathrm{OH}$

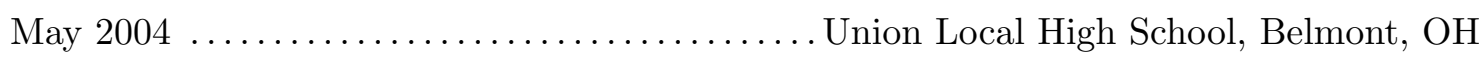

July $2006 \ldots \ldots \ldots \ldots \ldots \ldots \ldots \ldots \ldots \ldots \ldots \ldots \ldots \ldots$ NASA Undergraduate Student Research Program, Marshall Space Flight Center, Huntsville, AL

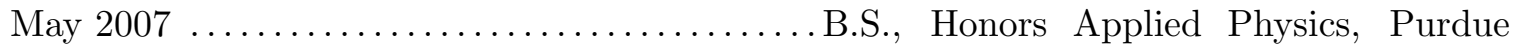
University, West Lafayette, IN

September 2007- March 2008 ............. GTA, The Ohio State University, Columbus, $\mathrm{OH}$

March 2008 - December 2012 ............. GRA, The Ohio State University, Columbus, $\mathrm{OH}$

August 2009 ............................. Physics, The Ohio State University, Columbus, $\mathrm{OH}$

June 2011 - June 2013 ................... Lawrence Scholar, Lawrence Livermore National Laboratory, Livermore, CA

\section{Experiments}

June $2008-8$ weeks $\ldots \ldots \ldots \ldots \ldots \ldots \ldots \ldots$ LLNL, Jupiter Laser Facility, Titan experiment, Principle Investigator P. Patel (LLNL)

August $2009-5$ weeks $\ldots . \ldots \ldots \ldots \ldots \ldots \ldots$. LLNL, Jupiter Laser Facility, Titan experiment, Principle Investigator Y. Ping (LLNL)

March $2010-5$ weeks $\ldots \ldots \ldots \ldots \ldots \ldots \ldots \ldots$.............. Jupiter Laser Facility, Titan experiment, Principle Investigator Y. Ping (LLNL) 
August $2010-5$ weeks LLNL, Jupiter Laser Facility, Titan experiment Principle Investigator R. Fedosejevs (University of Alberta)

February $2011-4$ weeks $\ldots \ldots \ldots \ldots \ldots \ldots$. LLNL, Jupiter Laser Facility, Titan experiment, Principle InvestigatorI M. Wei (General Atomics)

May $2011-3$ weeks ....................LLL, Jupiter Laser Facility, Titan experiment, Principle Investigator A. Link (LLNL)

August $2011-4$ weeks $\quad \ldots \ldots \ldots \ldots \ldots \ldots \ldots$. LLNL, Jupiter Laser Facility, Titan experiment Principle Investigator R. Fedosejevs (University of Alberta)

November $2011-1$ week $\ldots . \ldots \ldots \ldots \ldots \ldots$........... Jupiter Laser Facility, Titan experiment, Principle Investigator Hui Chen (LLNL)

March 2012 - 5 weeks $\ldots \ldots \ldots \ldots \ldots \ldots \ldots$. LANL, Trident experiment, Principle Investigators C. McGuffey (UCSD) and A. Link (LLNL)

June $2013-1$ week $\ldots \ldots \ldots \ldots \ldots \ldots \ldots$. University of Rochester, LLE, MTW SpecFROG setup, Principle Investigators Y. Ping (LLNL) and A. Link (LLNL)

\section{Publications}

\section{Papers in Print}

G. E. Kemp, A. Link, Y. Ping, D. W. Schumacher, R. R. Freeman and P. K. Patel, Coupling of laser energy into hot-electrons in high-contrast relativistic laser-plasma interactions, Phys. Plasmas 20, 033104 (2013); doi: 10.1063/1.4794961

Y. Ping, et al, Dynamics of Relativistic Laser-Plasma Interaction on Solid Targets, PRL 109, 145006 (2012); doi:10.1103/PhysRevLett.109.145006

D. W. Schumacher, G. E. Kemp, A. Link, R. R. Freeman and L. D. Van Woerkom, The shaped critical surface in high intensity laser plasma interactions, Phys. Plasmas 18, 013102 (2011); doi:0.1063/1.3532958

V. M. Ovchinnikov, G. E. Kemp, D. W. Schumacher, R. R. Freeman and L. D. Van Woerkom, How well do time-integrated $K_{\alpha}$ images represent hot electron spatial distributions?, Phys. Plasmas 18, 072704 (2011); doi:10.1063/1.3609819 
V. M. Ovchinnikov, D. W. Schumacher, G. E. Kemp, A. G. Krygier, L. D. Van Woerkom, K. U. Akli, R. R. Freeman, R. B. Stephens and A. Link, Using time-integrated Ka images to study refluxing and the extent of pre-plasmas in intense laser-plasma experiment, Phys. Plasmas 18, 112702 (2011); doi:10.1063/1.3656957

F. Perez, et al, Single-shot divergence measurements of a laser-generated relativistic electron beam, Phys. Plasmas 17, 113106 (2010); doi:10.1063/1.3514595

K. U. Akli, et al, A dual channel X-ray spectrometer for fast ignition research, JINST 5 P07008 (2010) doi:10.1088/1748-0221/5/07/P07008

\section{Conference Publications}

M. S. Wei, et al, Study of Fast Electron Generation and Transport for Fast Ignition, International Atomic Energy Agency 24th Fusion Energy Conference (2013); IFE/P6-06

R. B. Stephens, et al, Divergence of laser-generated hot electrons produced in a cone geometry, J. Phys: Conf. Series 244, 022064 (2010); doi:10.1088/1742-6596/244/2/022064

K. U. Akli, et al, Hot electron generation and transport using Ka emission, J. Phys: Conf. Series 244, 022026 (2010); doi:10.1088/1742-6596/244/2/022026

I. Hrbud, G. E. Kemp, A. H. Yan and J. G. Gedrimas, Review of RF Plasma Thruster Development, 30th International Electric Propulsion Conference (2007), IEPC-2007-309

G. E. Kemp, J. W. Dankanich, G. R. Woodcock and D. R. Wingo, Solar Electric Propulsion Upper-Stage for Launch Vehicle Capability Enhancement, 54th Joint Army-NavyNASA-Air Force (JANNAF) Propulsion Meeting (2007), JSC CD-49

\section{Conference Talks}

G. E. Kemp, et al, Specular reflectivity and absorption in high contrast relativistic laserplasma interactions, 12th International Workshop on Fast Ignition of Fusion Targets (2012)

G. E. Kemp, et al, Specular reflectivity in high contrast relativistic laser-plasma interactions, 54th Annual Meeting of the APS Division of Plasma Physics, Volume 57, Number 12 (2012); Abstract J05.00003

G. E. Kemp, et al, Experimental and LSP modeling of pre-pulse effects on the laser-plasma interaction by a $527 \mathrm{~nm}$ laser pulse, 53rd Annual Meeting of the APS Division of Plasma Physics, Volume 56, Number 16 (2011); Abstract: BAPS.2011.DPP.TO6.12

G. E. Kemp, D. W. Schumacher, A. Link, R. R. Freeman and L. D. Van Woerkom, The Shaped Critical Surface in High Intensity Laser-Plasma Interactions, 52nd Annual 
Meeting of the APS Division of Plasma Physics, Volume 55, Number 15 (2010); Abstract: BAPS.2010.DPP.PO7.6

G. E. Kemp, J. W. Dankanich, G. R. Woodcock and D. R. Wingo, Solar Electric Propulsion Upper-Stage for Launch Vehicle Capability Enhancement, 54th Joint Army-NavyNASA-Air Force (JANNAF) Propulsion Meeting (2007), JSC CD-49

\section{Fields of Study}

Major Field: Physics 


\section{Table of Contents}

Page

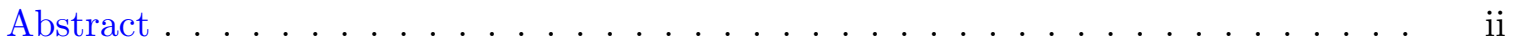

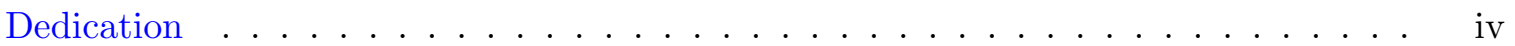

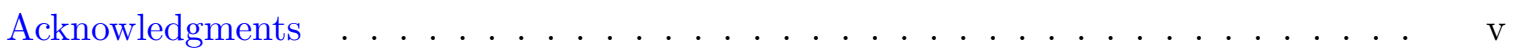

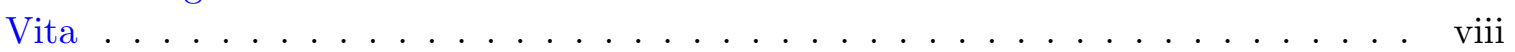

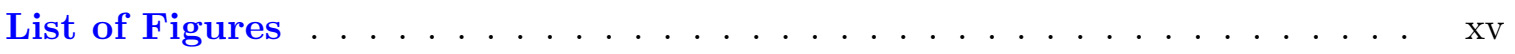

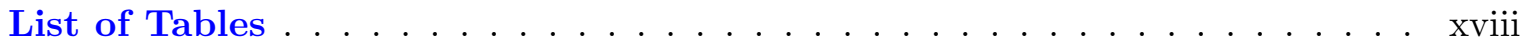

\section{Chapters}

1 Introduction to ultra-intense laser-plasma interactions . . . . . . . . 1

1.1 Motivation . . . . . . . . . . . . . . . . 1

1.2 Issues typical of ultra-intense laser-plasma interactions . . . . . . . . . . 6

1.3 Principle objectives and outline . . . . . . . . . . . . . . 9

1.4 Units . . . . . . . . . . . . . . . . . . . . . . . 12

1.5 Physics fundamentals . . . . . . . . . . . . . . . . . . 12

1.5.1 Single electron interactions with electromagnetic fields . . . . . . . . 12

1.5.2 Plasma fundamentals . . . . . . . . . . . . . . . . 18

1.5.3 Electromagnetic waves in plasma . . . . . . . . . . . . . 21

1.5.4 Non-linear and relativistic laser-plasma phenomena . . . . . . . . . . 22

1.5.5 Laser-driven quasi-static magnetic field generation . . . . . . . . . . 27

1.5.6 Over-dense interactions . . . . . . . . . . . . . . 30

1.6 Simulation fundamentals . . . . . . . . . . . . . . . . . . . 32

1.6.1 Introduction . . . . . . . . . . . . . . . . . 32

1.6.2 Direct implicit algorithm . . . . . . . . . . . . . . . . . 33

1.6.3 Implementation . . . . . . . . . . . . . . . 36

2 Low and high-contrast relativistic laser-plasma interactions experiment 38

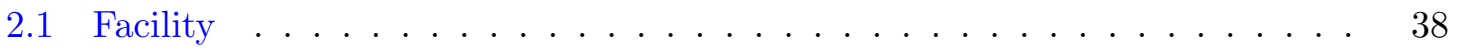

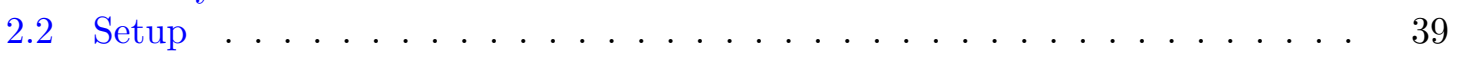

2.3 Typical pulse characteristics . . . . . . . . . . . . . . . . 44

2.4 Initial target conditions . . . . . . . . . . . . . . . . 50

2.4 .1 Low-contrast . . . . . . . . . . . . . . . 50

2.4 .2 High-contrast . . . . . . . . . . . . . . . . . . . . . . . 52

2.5 Specular pulse properties . . . . . . . . . . . . . 53

2.5.1 Integrated reflectivity and spectrum $\ldots \ldots \ldots 53$ 
2.5.2 Specular FROG measurements . . . . . . . . . . . . . . . . 61

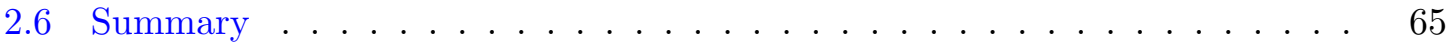

3 The shaped critical surface in low-contrast high-intensity laser plasma

interactions ......................... 69

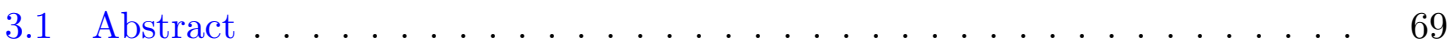

3.2 Introduction . . . . . . . . . . . . . . . . . 70

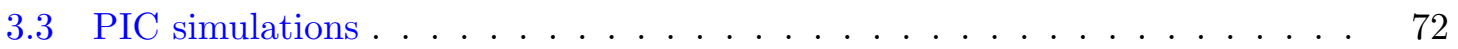

3.4 The shaped critical surface and a simple model . . . . . . . . . . . . 72

3.5 Results and discussion . . . . . . . . . . . . . . . . 76

3.6 A new diagnostic and summary . . . . . . . . . . . . . . . 79

4 Dynamic ionization in low-contrast relativistic laser-plasma interactions 81

4.1 PIC simulations . . . . . . . . . . . . . . . . . 82

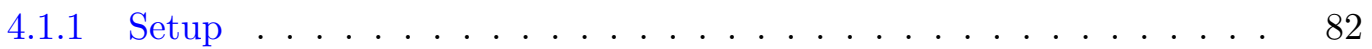

4.1.2 Interaction region evolution . . . . . . . . . . . . . 84

4.2 Near-field physics and far-field specular reflectivity measurements . . . . . . 88

$4.2 .1 \quad$ Near-field physics . . . . . . . . . . . . . . . . 88

4.2.2 Analytic pulse propagation techniques . . . . . . . . . . . 91

$4.2 .3 \quad$ Far-field specular reflectivity . . . . . . . . . . . . . . 95

4.3 Hot-electron properties . . . . . . . . . . . . . . . . . . . 102

4.4 Summary . . . . . . . . . . . . . . . . . . . 107

5 Coupling of laser energy into hot-electrons in high-contrast relativistic laser-plasma interactions . . . . . . . . . . . . . . . 108

5.1 Abstract . . . . . . . . . . . . . . . . . . . 109

5.2 Introduction . . . . . . . . . . . . . . . . . . . . 109

5.3 Simulation setup . . . . . . . . . . . . . . . . . . . . . 112

5.4 Perfectly flat interface . . . . . . . . . . . . . . . . . . 115

5.5 Surface perturbations . . . . . . . . . . . . . . . . 120

5.5.1 Enhanced coupling from Weibel-like fields . . . . . . . . . . . 120

5.5.2 Under-dense acceleration . . . . . . . . . . . . . . . . . 124

5.5.3 Quasi-static fields vs. wave-front perturbations . . . . . . . . . . 127

5.6 Summary . . . . . . . . . . . . . . . . . . . . . 129

6 Specular reflectivity and absorption in high-contrast relativistic laser-

plasma interactions . . . . . . . . . . . . . . . . . 132

6.1 PIC simulations . . . . . . . . . . . . . . . . . . . . . . 134

6.1 .1 Setup . . . . . . . . . . . . . . . . . . 134

6.1 .2 Interaction region evolution . . . . . . . . . . . . . . 137

6.2 Specular reflectivity . . . . . . . . . . . . . . . . . . . . 139

6.2 .1 Near-field . . . . . . . . . . . . . . . . . . . . . . . . 139

6.2 .2 Far-field . . . . . . . . . . . . . . . . . . . . . . 142

6.3 Hot-electron sensitivity to surface features . . . . . . . . . . . . . . . . 149

6.3.1 Short pulse parameter scan . . . . . . . . . . . . . . . 149

6.3 .2 Long pulse differences . . . . . . . . . . . . . . . . . . . . . 150

6.4 Summary . . . . . . . . . . . . . . . . . . 153 


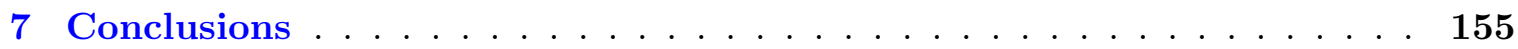

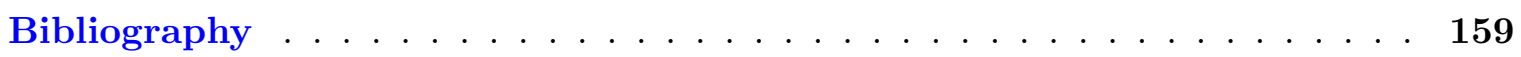

\section{Appendices}

A Laser Diagnostic Table Layout . . . . . . . . . . . . . . . 180

B Pre-pulse Monitor . . . . . . . . . . . . . . . . . . . 181

C Short pulse measurement: Frequency Resolved Optical Gating . . . . 182

C.1 Single-shot, polarization-gated FROG . . . . . . . . . . . . . . . 184

C.2 Pulse retrieval . . . . . . . . . . . . . . . . . . . . . . . . 187

C.3 Preparing the data for retrieval . . . . . . . . . . . . . . . . . 190

C.4 Error analysis . . . . . . . . . . . . . . . . . . . . . . . 191

D SpecFROG measurements on OMEGA EP . . . . . . . . . . 193

D.1 Principal objectives of the proposed research . . . . . . . . . . . 193

D.2 Background information . . . . . . . . . . . . . . . . . . 194

D.3 Experimental design and approach . . . . . . . . . . . . . . 195

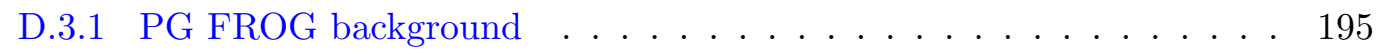

D.3.2 Requirements . . . . . . . . . . . . . . . . . . 197

D.3.3 Design . . . . . . . . . . . . . . . . . . . . . . . . . . . . . 198

D.3.4 Campaign, support and simulations _. . . . . . . . . . 200

D.4 Expected results; significance and/or application of expected results _ . . . 201

D.5 Relevance of proposed research to facilitys programs and goals . . . . . . 201

D.6 Aspects of the facility which provide unique opportunities for conducting the proposed research . . . . . . . . . . . . . . . . . 202 


\section{List of Figures}

Figure

Page

1.1 Astrophysical regimes of warm-dense matter . . . . . . . . . . . . . . 2

1.2 Approaches to inertial confinement fusion . . . . . . . . . . . . . . 4

1.3 Illustration of target normal sheath acceleration . . . . . . . . . . 5

1.4 Calculations of energy-angle phase-space of electrons accelerated by a planewave . . . . . . . . . . . . . . . . . . . . 15

1.5 Schematic of over-the-barrier field ionization . . . . . . . . . . 17

1.6 Ionization potentials of common materials . . . . . . . . . . . . 18

1.7 Illustration of plasma density oscillations . . . . . . . . . . . . . . 20

1.8 Spectral modifications to a laser pulse propagating through plasma . . . . . 24

1.9 Temporal and spatial envelope modification of a relativistic laser pulse in under-dense plasma . . . . . . . . . . . . . . 26

1.10 Gyroradius of electrons in a uniform external magnetic field . . . . . . . . 28

1.11 Sources of quasi-static magnetic field generation in relativistic LPI . . . . . 29

1.12 Traditional sharp, supra-critical plasma acceleration schemes . . . . . . . 31

1.13 Outline of direct-implicit algorithm in LsP . . . . . . . . . . . . . 34

2.1 Experiment: goals . . . . . . . . . . . . . . . . . . . 40

2.2 Experiment: target geometry . . . . . . . . . . . . . . . 40

2.3 Experiment: picture of Titan target area . . . . . . . . . . . . . . 42

2.4 Experiment: setup . . . . . . . . . . . . . . . . . 42

2.5 Experiment: typical pre-pusle measurement . . . . . . . . . . . . . . 44

2.6 Experiment: typical laser FROG trace best . . . . . . . . . . . . . . 46

2.7 Experiment: typical laser FROG trace ave . . . . . . . . . . . . . . 47

2.8 Experiment: estimated laser focal spot . . . . . . . . . . . . . . . 49

2.9 Experiment: modeled pre-plasma from MULTI2D . . . . . . . . . . . 51

2.10 Experiment: target surface roughness f . . . . . . . . . . . 54

2.11 Experiment: Lambertian scatterer . . . . . . . . . . . . . . 55

2.12 Experiment: Spectralon ${ }^{\circledR}$ filters and calibration . . . . . . . . . . 56

2.13 Experiment: comparison of spectra . . . . . . . . . . . . . 57

2.14 Experiment: low-contrast specular reflectivity data . . . . . . . . . . 59

2.15 Experiment: high-contrast specular reflectivity data . . . . . . . . . . 60

2.16 Experiment: reflectivity from grating-like targets . . . . . . . . . . . 61 
2.17 Experiment: low-contrast specular FROG data best . . . . . . . . . . 63

2.18 Experiment: low-contrast specular FROG data ave . . . . . . . . . . . . . . 64

2.19 Experiment: high-contrast specular FROG data best . . . . . . . . . . 65

2.20 Experiment: high-contrast specular FROG data ave . . . . . . . . . 66

3.3 Cartoon depicting shaped critical surface and simple reflectivity model . . . 75

3.4 Spatial intensity distribution of specularly reflected pulse $4 \mathrm{~mm}$ away from

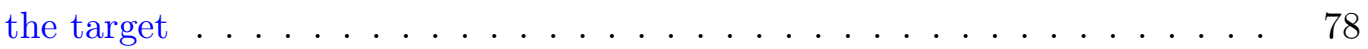

3.5 Sensitivity of reflectivity to propagation through under-dense plasma . . . . 79

4.1 Low-contrast specular reflectivity and absorption simulation setup . . . . . 83

4.2 Simulated evolution of electron density, ion charge state and laser intensity for the low-contrast interaction with fixed charge state . . . . . . . . 86

4.3 Simulated evolution of electron density, ion charge state and laser intensity for the low contrast interaction with dynamic ionization . . . . . . . . .

4.4 Differences in near-field estimates of spectral shifting for the low-contrast simulations between fixed and field ionization . . . . . . . . . . . 90

4.5 Spatial spectral intensity distribution of a specularly reflected pulse . . . . 95

4.6 Phase-front distortions in the specularly reflected pulse due to surface features 96

4.7 Integrated specular reflectivity measurements of the fixed and dynamic ionization simulations with Multi2D pre-plasma . . . . . . . . . . . . 98

4.8 Spatially and temporally resolved intensity and spectral shifts of the lowcontrast specularly reflected pulse . . . . . . . . . . . . . . 100

4.9 Intensity weighted near-field and far-field instantaneous spectral shifts of the low-contrast specularly reflected pulse . . . . . . . . . . . . . 101

4.10 Integrated electron energy spectra for low-contrast interaction . . . . . . . . 103

4.11 Integrated hot-electron energy-anler distributions for low-contrast interactions 104

4.12 Quasi-static magnetic fields for fixed and field ionized pre-plasmas . . . . . 106

5.1 PIC simulation geometry for high-contrast LPI with targets with sinusoidal surface features . . . . . . . . . . . . . . . . . . . . . 113

5.2 Temporal evolution of perfectly flat target interaction . . . . . . . . . . 116

5.3 Calculations of hot-electron energy-angle phase-space created by standing wave accelerations . . . . . . . . . . . . . . . . . 118

5.4 Temporal evolution of optically flat target interaction . . . . . . . . . . 121

5.5 Typical electron trajectory characteristics of perfectly and optically flat interfaces .......................... 123

5.6 Hot-electron energy distributions of various sinusoidal surface perturbations

5.7 Temporal evolution of interaction with sinusoidal surface perturbations where critical surface motion smooths over features . . . . . . . . . . . . . 126

5.8 Calculations of hot-electron energy-angle phase-space created by travelingwave accelerations . . . . . . . . . . . . . . .

5.9 Temporal evolution of interaction with sinusoidal surface perturbations where critical surface motion does not smooths over features . . . . . . . . . 129

6.1 High-contrast specular reflectivity and absorption simulation setup . . . . . 135

6.2 Variety of reconstructed surface perturbations considered for the simulations 136 
6.3 Simulated evolution of electron density, ion charge state and laser intensity for the high-contrast interaction with dynamic ionization . . . . . . . . 138

6.4 Estimated spectral shifting of the high-contrast specularly reflected pulse . 140

6.5 Comparison of near and far-field instantaneous specular pulse properties, resolved in space and time . . . . . . . . . . . . . . . . . . 143

6.6 Comparison of near and far-field instantaneous specular pulse properties, resolved in time . . . . . . . . . . . . . . . . . . . . . 144

6.7 Comparison of short and long pulse spectra and instantaneous wavelength shift145

6.8 Comparison of short and long pulse, time-integrated, spatial intensity profiles of the specularly reflected pulse in both the near and far-field . . . . . . . . 147

6.9 Influence of large and small scale features in far-field reflectivity for the high contrast $100 \mathrm{fs}$ pulse . . . . . . . . . . . . . . . . . . 148

6.10 Hot-electron spectra generated from various experimentally inspired surfaces 151

6.11 Angular distribution of hot-electrons for various experimentally inspired surface perturbations . . . . . . . . . . . . . . . 152

6.12 Long pulse high-contrast electron source characteristics . . . . . . . . . 153

7.1 Comparison of low and high-contrast hot-electron normalized angular distributions ........................... 156

7.2 Comparison of low and high-contrast hot-electron energy spectra . . . . . . 158

A.1 Experiment: laser diagnostic table layout . . . . . . . . . . . . . . . 180

B.1 Experiment: pre-pulse monitor . . . . . . . . . . . . . . . . 181

C.1 FROG: single-shot PG configuration . . . . . . . . . . . . . . . . . 184

C.2 FROG: Asymmetric mixing . . . . . . . . . . . . . . . . . . 185

C.3 FROG: PCGP pulse retrieval algorithm . . . . . . . . . . . . 188

D.1 SpecFROG: experimental trace from Titan . . . . . . . . . . . . 196

D.2 SpecFROG: schematic of transport optics . . . . . . . . . . . . . 199

D.3 SpecFROG: sketch of proposed FROG . . . . . . . . . . . . . . . . 199 


\section{List of Tables}

Table

Page

2.1 Typical Titan pulse characteristics. . . . . . . . . . . . . . . . . 39

2.2 Experiment: summary of MULTI2D modeling of pre-plasma environment . 51

2.3 Experiment: summary of specular diagnostic observables . . . . . . . . . 66

5.1 Conversion efficiencies of various sinusoidal surface perturbations . . . . . . 113

5.2 Composite conversion efficiencies of various sinusoidal surface perturbations 125

6.1 Hot-electron conversion efficiency for the various experimentally realistic surface perturbations . . . . . . . . . . . . . . . . 149

C.1 FROG: Popular geometries _. . . . . . . . . . . . . . 183 


\section{Chapter 1}

\section{INTRODUCTION TO ULTRA-INTENSE LASER-PLASMA INTERACTIONS}

\subsection{Motivation}

Ultra-intense laser $\left(>10^{18} \mathrm{~W} / \mathrm{cm}^{2}\right)$ interactions with matter are capable of producing relativistic electrons which have a variety of applications ranging from generating extreme states of matter [1,2], creating short duration, directional x-ray and $\gamma$-ray sources [3], creating high density electron-positron plasmas [4] and hadron cancer treatment research [5]. Each have very stringent requirements on the energy spectrum, angular distribution and conversion efficiency where control of various aspects of these relativistic electron distributions is essential to their effectiveness. Some of the more relevant applications for the work in this thesis include warm-dense matter generation, the cone guided fast ignitor (FI) approach to inertial confinement fusion (ICF), and ion acceleration.

\section{Warm-dense matter generation}

Warm-dense matter (WDM) is the region of phase-space between condensed matter and plasma where systems have energy densities of $10^{11} \mathrm{~J} / \mathrm{m}^{3}$. Understanding how materials behave in this regime is essential to studying the evolution of many astrophysical phenomena $[6,7]$. The equation of state (i.e. the correlation between density, temperature and pressure) of hydrogen, for example, at extreme densities and pressures like those present at the center of giant gas planets like Jupiter (Figure 1.1(a)) determines whether the core is liquid or solid and how the planet's magnetic field is produced. Stellar phenomena at these extremes, such 
as supernova (Figure 1.1)(b), astrophysical jets and conditions present in the Sun (Figure 1.1(c)), are also driving development and improvement of x-ray opacity, radiation energy transport and atomic physics models used to describe equilibrium processes and the long term evolution of the system. This research is also fundamental to understanding how matter behaves at the early stages of fusion [8] and designing controlled reactions within a laboratory environment such as the National Ignition Facility (NIF) [9].

(a) Jupiter

(b) G299.2-2.9 supernova remnants (c) The Sun

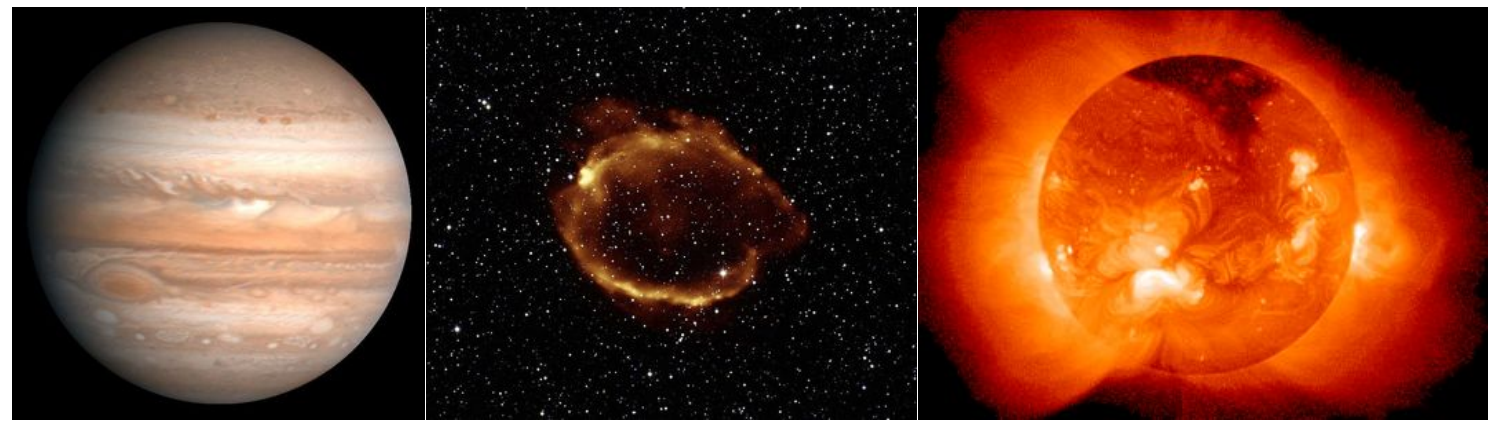

Figure 1.1: Astrophysical regimes of interest to warm-dense matter generation: (a) equation-of-state models for hydrogen at high density and temperature, such as at the core of giant gas planets like Jupiter, evolution of radiation driven systems like (b) supernova and $(\mathrm{c})$ the sun.

Until recently, the experimental conditions required to study these phenomena and benchmark the models have not been readily accessible anywhere other than at enormous facilities, like NIF, the Sandia Z-machine [10], and LCLS [11]. With the advent of new excitation techniques, hot-electrons generated in 'university-scale' intense LPI experiments have been used to generate these high energy density (HED) conditions by isochorically heating reduced mass targets to tens of $k e V$ at near solid densities [2, 12, 13].

\section{Fast ignitor approach to inertial confinement fusion}

Ever since its inception in the early 70 's $[14,15]$, laser-induced thermonuclear fusion has been extensively studied for it's extremely appealing prospect of a clean energy source. 
Alternatively to magnetic confinement [16], which has been around for more than 50 years, this approach relies on the fuels own inertia to confine itself for the duration of the fusion burn ( 10s of picoseconds), dubbed inertial confinement fusion (ICF). In thermonuclear fusion, the energy to overcome the Coulomb force comes from heating the fuel to high temperatures, so the ideal fuel for the reaction would be deuterium-tritium (D-T, $D+T \rightarrow$ $\left.{ }_{2}^{4} \mathrm{He}(3.6 \mathrm{MeV})+n(14.1 \mathrm{MeV})\right)$ for its high nuclear cross section at 'moderate' temperatures (tens of $\mathrm{keV}$ ) and relative abundance in the oceans. High fuel density and temperatures, $\sim 1000 \times \operatorname{STP}$ (standard temperature and pressure) conditions at $\sim 10 \mathrm{keV}$, ensure an efficient and containable burn in a laboratory setting, but nearly isentropic compression of cryogenic, fermi-degenerate fuel $(\sim 11$ Kelvin $)$ is required to minimize compression driver energy [17].

Once compressed, the energy required to heat a solid sphere of this compressed fuel to $\sim 10 \mathrm{keV}$ to initiate the fusion burn, known as volume ignition, is not feasible for a self-sustaining reactor design [18]. The conventional isobaric approach, known as hot-spot ignition [19], has a low density D-T gas at the center of a hollow spherical shell of cryogenic fuel. The mechanical $P d V$ work done on the low density gas during the compression stage simultaneously heats the hot-spot to ignition conditions, generating energetic $\alpha$-particles which then heat the surrounding compressed D-T fuel and propagate the burn from the inside-out until the fuel is consumed [8]. The whole process is proposed to work in four stages [17], depicted in Figure 1.2(a). (i) In the first stage, either direct laser [20, 21] or indirect x-ray radiation [8] ablates away the outer portion of the cryogenic fuel shell. (ii) The heated coronal plasma expands and, by momentum conservation, simultaneously compresses the cryogenic shell and the low density gas at the core. (iii) The hot-spot forms from the compressed gas core, ignites and (iv) the burn propagates outward, consuming the cold fuel and eventually exploding.

Hot-spot ignition, however, is plagued by hydrodynamic instabilities [22], driver asymmetries [23] and species separation [24], all of which lead to less than ideal thermonuclear yields. The electron fast ignition approach [1], alternatively, suggests that a short pulse, PetaWatt class laser $(10 s p s, 10 s k J)$ could be used to generate a supra-thermal electron 
(a) Hot spot ignition, inertial confinement fusion

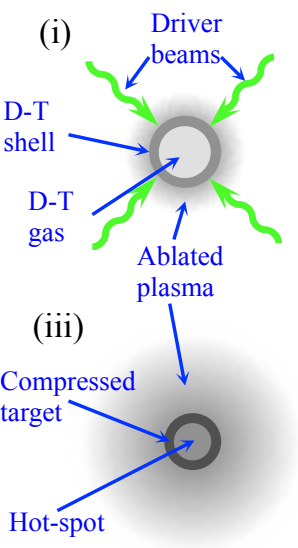

(ii)

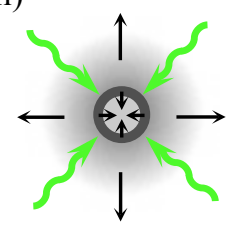

(iv)

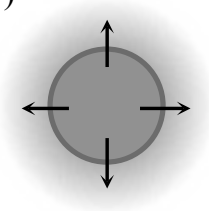

(b) Cone guided fast ignition

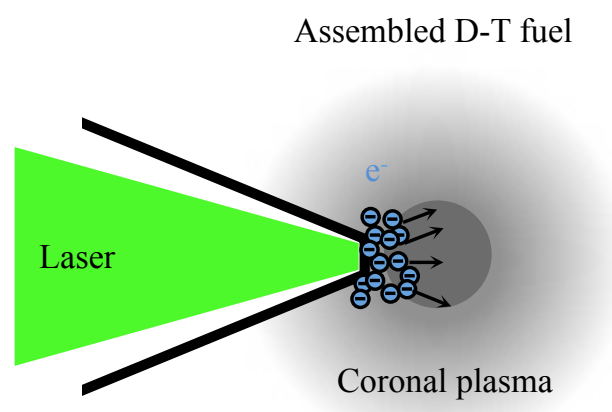

Figure 1.2: (a) Four stages of hot-spot ignition inertial confinement fusion, adapted from [17]: (i) driver energy vaporizes ablative layer, (ii) plasma expands and spherical implosion compresses cold fuel along with generating a hot spot, (iii) hot spot forms and ignites the fuel and (iv) burn propagates outward consuming the cold fuel and eventually exploding. (b) Cone guided fast ignitor concept.

beam that would rapidly (i.e. isochorically) heat the compressed target. In theory, FI would simultaneously provide higher fusion gains and lessen drive symmetry requirements and hydrodynamic instabilities that plague traditional hot-spot ICF. The coronal plasma created by the compression stage of the target, however, can extend over milimeters and would push critical electron density, the point at which electromagnetic waves can no longer propagate in plasma $\left(\approx 10^{21} e^{-} / \mathrm{cm}^{3}\right)$, several $100 \mu \mathrm{m}$ from the compressed core and with it the hot-electron source. Given the offset distance of the hot-electron source from the compressed fuel core, this electron beam would ideally need to be be nearly collimated and mono-energetic (1-3 $\mathrm{MeV})$ such that a significant fraction of the electron energy would deposited into the compressed fuel. Unfortunately, in reality, this assumption departs quite drastically from both recent experiment [25-28] and simulation [29-33] work.

It was originally suggested the laser-hole boring [34], the process by which radiation pressure steepens the electron density profile, would push the hot-electron source closer to the compressed fuel and minimize the offset distance but recent measurements have suggested that this motion may be limited by heated plasma expansion [35](In an upcoming 
LBS experiment on OMEGA EP at the Laboratory for Laser Energetics (FY13), we will repeat this measurement at FI relevant conditions. Some of these details are discussed in Appendix D). In cone guided fast ignition, depicted in Figure 1.2(b), a cone is used to provide an evacuated path, free of the coronal plasma from the compression stage, for the laser to propagate, placing the hot-electron source closer to the compressed core.

\section{Ion acceleration with target normal sheath acceleration}

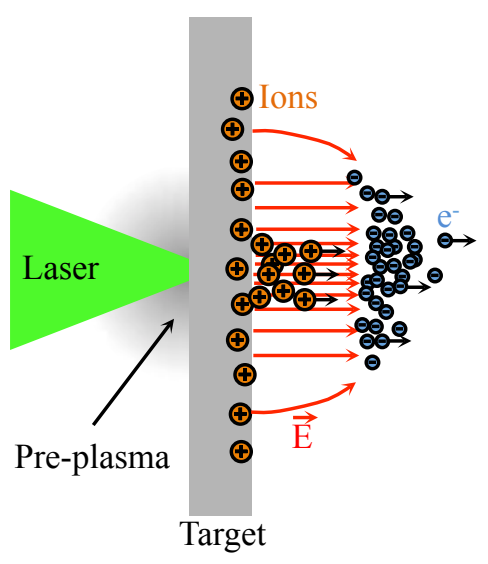

Figure 1.3: Illustration of target normal sheath acceleration. High energy electrons generated by the intense LPI transverse and escape the target, setting up $M V / \mu m$ electrostatic sheath fields near the surface which then accelerate protons and heavier ions to multi-MeV energies by this field. Figure adapted from [36].

Directed energetic protons and ions, because of their highly localized energy deposition near the end of their travel and nearly ballistic trajectories, have also been suggested as an alternative to electrons for WDM creation [2] and fast-ignition [37, 38]. Theoretically, these high energy hadrons also have medical applications a superior form of cancer treatment compared to traditional photon based approaches [5]. Alternatively to the massive cyclotron particle accelerators, ions can be accelerated using relativistic electrons generated in intense laser-plasma interactions (LPI) via a process known as target normal sheath acceleration 
(TNSA) [39, 40], illustrated in Figure 1.3. The most energetic electrons escape the laser interaction region, traverse the target and escape into vacuum, charging the target and creating $\sim M V / \mu m$ electrostatic sheath fields normal to the surface. These fields then ionize the target and the resulting ions are accelerated to multi $-\mathrm{MeV}$ energies, predominantly coupling into hydrocarbon contaminants on the rear surface of the target [41].

\subsection{Issues typical of ultra-intense laser-plasma interactions}

\section{Laser pulse contrast}

Unless painstaking measures are taken, these ultra-intense short-pulse lasers are typically preceded by millijoules (if not more) of laser energy nanoseconds before the main pulse arrives at the target, dubbed 'pre-pulse,' often with sufficient intensity to create $10 \mathrm{~s}$ of microns of under-dense plasma in front of the target before the short pulse of interest arrives. The pulse then must propagate through this 'pre-plasma' before being reflected at the critical density interface, becoming subject to non-linear instabilities that result in spectral [42], temporal [43] and spatial [44] distortions, creating significantly different pulse characteristics and driving additional (often less predictable) shot-to-shot variations. The presence of pre-plasma also introduces additional magnetic field generation mechanisms [4446], resulting in quasi-static hundreds of MegaGauss fields that can extend over microns and persist throughout the interaction which can perturb, or even trap, relativistic electron trajectories.

High-contrast lasers minimize the laser energy before the main pulse, minimizing the often detrimental effects of pre-plasma on the propagating laser pulse and hot-electron generation and transport. Several facilities, like LULI at École Polytechnique (France) [47], Trident at Los Alamos National Laboratory (NM) [48], HERCULES at the University of Michigan (MI) [49], Titan at Lawrence Livermore National Laboratory (CA) [50], OMEGA EP at the Laboratory for Laser Energetics (NY) [51], Orion at the Atomic Weapons Establishment (UK) [52] and, of course, Scarlet at The Ohio State University (OH) [53] already have this pulse cleaning capability typically obtained through nonlinear optical processes, 
such as harmonic generation and third order cross-polarized wave generation [54], or with plasma mirrors $[55,56]$.

Correspondingly, experiments and simulations have seen marked improvements in performance by increasing the laser pulse contrast. In WDM generation, it was found that a significant fraction of the hot-electrons generated by the laser was lost to heating the pre-plasma (due to a strongly magnetized under-dense plasma) and that switching to highcontrast pulses improved coupling to the bulk, resulting in increased target temperatures [13]. Recent experiments and laser-plasma simulations studying electrons generated with the cone-guided fast ignitor configuration have seen decreased angular spread $[57,58]$ and increased coupling of laser energy into electrons of interest to FI [59,60] with increasing contrast. In TNSA, sharp target interfaces are important for creating large sheath fields [39] and thiner targets allow for more escaping electrons with higher mean and maximum energy [61], but significant pre-pulse can create microns of under-dense pre-plasma or even destroy too thin of a target. The advent of high-contrast pulses has opened up the possibility of shooting sub-micron thick targets, resulting in increased maximum ion energies [62].

The advent of laser systems with intense, but extremely clean, laser pulses has ushered in a new and exciting regime of experiments. However, the absence of pre-plasma tends to reduce the overall laser-coupling efficiency and, in general, the coupling mechanisms are not well understood. Firstly, the coupling mechanisms are different with high-contrast pulses since any acceleration mechanisms must now be able to move electrons from high density regions into the vacuum laser fields before they can receive a relativistic kick. Under these conditions, the interaction region also more closely resembles the initial target interface thus making initial surface conditions important [63]. Additionally, accurate modeling the interaction at the sharp, supra-critical density interface is required to interpret any experimental results. 


\section{Experimental technique limitations}

To date, experiments are incapable of directly measuring the hot-electrons generated in intense laser-plasma interactions at the source, but many indirect measurements exist that can constrain the simulations used to infer the hot-electron properties. Transport diagnostics typically study the electrons as they propagate through the target by measuring bremsstrahlung emission [27, 28, 64], $K_{\alpha}$ emission from tracer fluors $[25,65]$ or wires $[59,66]$ generated when hot-electrons knock out k-shell electrons, and transition radiation at an interface [67] while other measurements directly measure electrons that escape the target using magnetic spectrometers [68, 69]. Electron transport (and measurements), however, can be quite sensitive to target geometry and refluxing [70], target heating [71], current [72] and resistivity gradient [73] driven magnetic fields inside the target, target charging effects [74] and filamentation instabilities [75]. Additionally, comparison of these simulations to experimental data are often limited by incomplete equations of state (EOS), emission models and opacities of these extreme states of matter, the difficulty of self-consistently modeling all essential aspects of the LPI and electron/photon transport in a single simulation [70], and computational limitations associated with 2D and 3D simulations for absolute comparison with experimental measurables [76].

Although often neglected in these experiments, the unabsorbed light (from both the specularly reflected pulse and harmonics generated near the interaction interface [77]) can provide a direct measurement of the laser-plasma interaction and previous work has suggested that it can be quite informative of the pre-plasma environment. ${ }^{1}$ The change in divergence between the incident and specularly reflected pulse, due to the shape of the relativistic critical surface, has been shown to be a strong indicator of pre-plasma scale length near critical density [78]. Instantaneous spectral shifting and broadening due to motion of the critical surface [35] and relativistic effects [42] have been observed to be quite sensitive to pre-plasma environment, as well as temporal pulse front steepening due to group velocity dispersion [43]. Spatial, spectral and polarimetry measurements of harmonics generated

${ }^{1}$ Albeit, a significant amount of additional work and equipment is needed since both the incident and specular pulses must be simultaneously characterized in the same manner. 
near the critical surface have been found to sensitive to pulse contrast [79] and target surface roughness $[80,81]$ as well as indicative of magnetic fields in the under-dense pre-plasma environment $[82,83]$.

\subsection{Principle objectives and outline}

The principle objective of this work is to qualitatively and quantitatively address how practical and/or effective experimentally obtained specular reflectivity measurements are at constraining simulations used to indirectly study relativistic electron generation in both low and high-contrast relativistic laser-plasma interactions. Experimental reflectivity measurements are, however, exclusively obtained in the 'far-field' where diffraction effects can potential to convolve the interpretation of the 'near-field' physics to the point of being useless. Note: in contrast with traditional definitions [84], 'near-field' in this thesis refers to the fields near the interaction region and 'far-field' refers to the fields far away from the interaction region, where diffraction effects have fully manifested themselves. For these farfield measurements to be useful, diffraction effects must be either able to be de-convolved or the far-field measuremetns must be indicative of the near-field interaction physics: to accomplish the principle objective of this work, the simulation work must be able to accommodate a comparison between the near-field physics and far-field measurements. The simulations, once reasonably constrained by the experimental reflectivity measurements, will be ultimately used to infer properties of the relativistic electron sources born in both low and high-contrast interactions. In the remainder of this chapter (Chapter 1.5), I discuss some background physics fundamental to understanding the material in this thesis.

State-of-the-art simulations used to study these laser-plasma interactions require a variety of algorithms to handle the sweeping range of plasma densities (from vacuum to solid density), electromagnetic wave propagation and interaction with matter, transport and generation of radiation and charged particles in solid density plasmas, equation-of-state of warm-dense matter, as well as vast computational resources to tie it all together. In Chapter 1.6, I briefly address some fundamental algorithms and difficulties associated with modeling 
this scale of laser-plasma interaction and assumptions made for computational convenience.

In the next chapter, I discuss specular reflectivity measurements from the experimental work that inspired this research. I discuss the experiment in Chapter 2, which compared high and low-contrast pulse interactions with solid density, planar aluminum targets. Spatial, temporal and spectral properties of both the incident and specularly reflected pulse are simultaneously obtained on each-and-every shot, a critical distinction from other similar work due to the shot-to-shot fluctuations in the incident laser properties. Significant differences in specular properties are observed between low and high-contrast laser conditions, with high-contrast conditions showing an additional sensitivity to different initial target surface perturbations.

For the low-contrast interactions, I continue the discussion with the influence of preplasma on the specularly reflected pulse using simulations with simplified pulses and fully ionized, exponential pre-plasma electron density profiles in Chapter 3. The shape of the relativistic critical surface plays a significant role in the far-field intensity distribution, at a distance where diffraction has fully manifested itself, which is observed to be indicative of the pre-plasma scale length. In Chapter 4, I discuss how laser-plasma instabilities associated with laser and target conditions more characteristic of the experiment can influence farfield specular reflectivity measurements and how quasi-static magnetic fields generated in the interaction can influence hot-electron generation.

For the high-contrast interactions, I begin in Chapter 5 with a discussion of hot-electrons acceleration mechanisms typical of these clean, but rough, interfaces using highly simplified high-contrast pulses and sinusoidal surface perturbations. The scale of the perturbations determine the evolution of the interaction interface, under-dense plasma expansion and ensuing hot-electron conversion efficiency, energy and angular distributions. I find that electron accelerations are dominated by the standing-wave that forms near the interface from the high reflectivity of the sharp interface. Following up this discussion, I next discuss realistic surface perturbations, using experimental measurements to constrain the specular reflected light properties and infer some properties of the resulting hot-electron source in Chapter 6. 
Finally, in Chapter 7, I conclude with a summary of the more relevant experimental and simulation findings. 


\subsection{Units}

All units in this thesis are Gaussian units (i.e. cgs) where length is measured in centimeters $[\mathrm{cm}]$, mass in grams $[\mathrm{g}]$ and time in seconds $[\mathrm{s}]$ unless otherwise stated. Some of the most useful quantities are

$\begin{array}{lll}\text { Quantity } & \text { Symbol } & \text { Value/Units } \\ \text { Electron charge } & -e & -4.80320428 \times 10^{-10} \mathrm{esu} \\ \text { Electron mass } & m_{e} & 9.10938215 \times 10^{28} \mathrm{~g} \\ \text { Speed of light, vacuum } & c & 2.99792458 \times 10^{10} \mathrm{~cm} / \mathrm{s} \\ \text { Boltzmann constant } & k_{B} & 1.380648813 \times 10^{-16} \mathrm{erg} / \mathrm{K} \\ \text { Electric field } & \vec{E} & \text { statV } / \mathrm{cm} \\ \text { Magnetic field } & \vec{B} & G\end{array}$

\subsection{Physics fundamentals}

In this section, we briefly discuss a few aspects of laser-plasma interactions that are fundamental to understanding the material in this thesis. First, we start with single electron interactions with relativistic electromagnetic plane-wave. This is followed by discussion of the collective behavior of plasmas and its influence on electromagnetic radiation. How the laser interacts with this plasma at sub-critical density is discussed next, along with several important non-linear phenomena and magnetic field generation mechanisms which can affect hot-electron generation and transport. Finally, several traditional high-contrast acceleration mechanisms are discussed.

\subsubsection{Single electron interactions with electromagnetic fields}

It is often useful to characterize an electromagnetic field using a vector potential $\vec{A}$, where the electric field $\vec{E}=-c^{-1} \partial \vec{A} / \partial t$ and magnetic field $\vec{B}=\nabla \times \vec{A}$. A plane wave traveling along the $\mathrm{x}$-axis $(\hat{x})$ with arbitrary polarization can be described by $\vec{A}=A_{o}\left[\delta \cos \phi \hat{y}+\sqrt{1-\delta^{2}} \sin \phi \hat{z}\right]$ where the phase $\phi=\omega_{L} t-k_{L} x$ and $\delta=0, \pm 1$ for 
linearly polarized light and $\delta= \pm 1 / \sqrt{2}$ for circularly polarized light, with the angular frequency of the light $\omega_{L}=c k_{L}=2 \pi c / \lambda_{L}$ and $c=2.998 \times 10^{10} \mathrm{~cm} / \mathrm{s}$ is the vacuum speed of light.

Following (but correcting) Gibbon [85], the solution for the motion of a single electron in this field start's with the Lorentz force

$$
\frac{d \vec{p}}{d t}=-e\left[\vec{E}+\frac{\vec{v}}{c} \times \vec{B}\right]
$$

where $\vec{p}=\gamma m_{e} \vec{v}$ is the electron's momentum with relativistic Lorentz factor $\gamma=\sqrt{1+p^{2} / m_{e}^{2} c^{2}},-e=-4.8 \times 10^{-10}$ esu and $m_{e}=9.11 \times 10^{-28} \mathrm{~g}$ are the electron charge and rest mass respectively. When combined with the work-energy theorem for an electron in an external EM field $\left(d\left(\gamma m_{e} c^{2}\right) / d t=-e \overrightarrow{v_{e}} \cdot \vec{E}\right)$, the vector potential and normalized units $\vec{a}=e \vec{A} / m_{e} c^{2}, \tau=\omega_{L} t, \chi=k_{L} x, \vec{\varrho}=\vec{p} / m_{e} c, \vec{\nu}=\vec{v} / c$, the equations of motion reduce to

$$
\begin{aligned}
\frac{d \varrho_{x}}{d \tau}-\frac{d \gamma}{d \tau} & =-\nu_{y}\left[\frac{\partial a_{y}}{\partial \tau}+\frac{\partial a_{y}}{\partial \chi}\right]-\nu_{x}\left[\frac{\partial a_{z}}{\partial \tau}+\frac{\partial a_{z}}{\partial \chi}\right] \\
\frac{d \varrho_{\perp}}{d \tau} & =\frac{\partial \vec{a}}{\partial \tau}+\nu_{x} \frac{\partial \vec{a}}{\partial \chi}
\end{aligned}
$$

where $\vec{\varrho}_{\perp}$ is the normalized momentum in the plane perpendicular to the laser propagation direction (i.e. $\left.\left(\varrho_{y}, \varrho_{z}\right)\right)$. The right hand side (RHS) of Equation 1.2 is identically equal to zero (since $\phi=\tau-\chi$ ) and the RHS of Equation 1.3 simply reduces to $d \vec{a}_{\perp} / d \tau$. Recalling that $\gamma^{2}=1+\varrho_{x}^{2}+\varrho_{\perp}^{2}$, the resulting electron momentum equations reduce to

$$
\begin{aligned}
& \varrho_{x}=\gamma-\alpha=\frac{1-\alpha^{2}+\varrho_{\perp}^{2}}{2 \alpha} \\
& \vec{\varrho}_{\perp}=\vec{a}+\vec{\varrho}_{\perp_{o}}
\end{aligned}
$$

where $\alpha$ is a constant of motion and $\vec{\varrho}_{\perp_{o}}$ an integration constant representing the initial transverse momentum. For an electron that starts from rest (i.e. $\varrho_{x_{o}}=\varrho_{\perp_{o}}=0$ at $\tau=\chi=0$ ), the momentum relations reduce to the classical ejection angle

$$
\tan \theta_{c}=\frac{\varrho_{\perp}}{\varrho_{x}}= \pm \sqrt{\frac{\gamma-1}{\gamma+1}}
$$


which is the angle the electron is traveling with respect to the laser propagation direction where, interestingly enough, this is only a function of the electron energy. Integration of the momentum equations results in the electron's position as a function of time which, in the center-of-rest frame, returns the characteristic 'figure-of-eight' motion.

More generalized expressions of the electron energy-angle relationship for arbitrary initial conditions can be obtained using Equations 1.4 and 1.5. For an electron injected into the field at $\tau=\chi=0$ with initial momentum $\vec{\varrho}_{o}=\left(\varrho_{x_{o}}, \varrho_{y_{o}}, \varrho_{z_{o}}\right)$, we find $\left.\vec{\varrho}_{\perp_{o}}=\left(\varrho_{y_{o}}-\delta a_{o}, \varrho_{z_{o}}\right)\right)$, $\alpha=\gamma_{o}-\varrho_{x_{o}}$ with corresponding momenta

$$
\begin{aligned}
\varrho_{x} & =\frac{1-\left(\gamma_{o}-\varrho_{x_{o}}\right)^{2}+\varrho_{y}^{2}+\varrho_{z}^{2}}{2\left(\gamma_{o}-\varrho_{x_{o}}\right)} \\
\varrho_{y} & =\varrho_{y_{o}}+\delta a_{o}(\cos (\phi)-1) \\
\varrho_{z} & =\varrho_{z_{o}}+\sqrt{1+\delta^{2}} a_{o} \sin (\phi)
\end{aligned}
$$

where $\gamma_{o}=\sqrt{1+\varrho_{o}^{2}}$. Shown in Figure 1.4 is the possible energy-angle phase space an electron point source can fill for a linearly polarized plane-wave with $a_{o}=3$ with wavelength $\lambda_{L}=527 \mathrm{~nm}$ and corresponding intensity of $4.4 \times 10^{19} \mathrm{~W} / \mathrm{cm}^{2}$ for electrons with various initial energies (colorscale) and orientation where electrons are injected at the (a) peak and (b) node electric field locations. ${ }^{2}$ The distributions are clearly 'bifurcated' with the separate 'fingers' always broadening with increasing initial electron energy with respect to the classical ejection angle (white curves).

The peak energy an electron, starting from rest, can gain in the oscillating electric field alone is given by the quiver energy $U_{p}=\left(\sqrt{1+a_{o}^{2}}-1\right) m_{e} c^{2}$ which for this field corresponds to $1.1 \mathrm{MeV}$; clearly the additional push from the $\vec{v} / c \times \vec{B}$ term with these relativistic conditions (i.e. $a_{o} \gtrsim 1$ ) is creating electrons with energy greater than the quiver energy. This occurs because electrons are very quickly accelerated to relativistic speeds along the laser propagation axis, allowing them to 'surf' with the field where they can gain more energy by staying 'in-phase' with the accelerating field for longer before being decelerated by the alternating field sign. Although the trajectories share a self-similar shape,

${ }^{2}$ These are conditions characteristic of the experiment discussed in Chapter 2. 


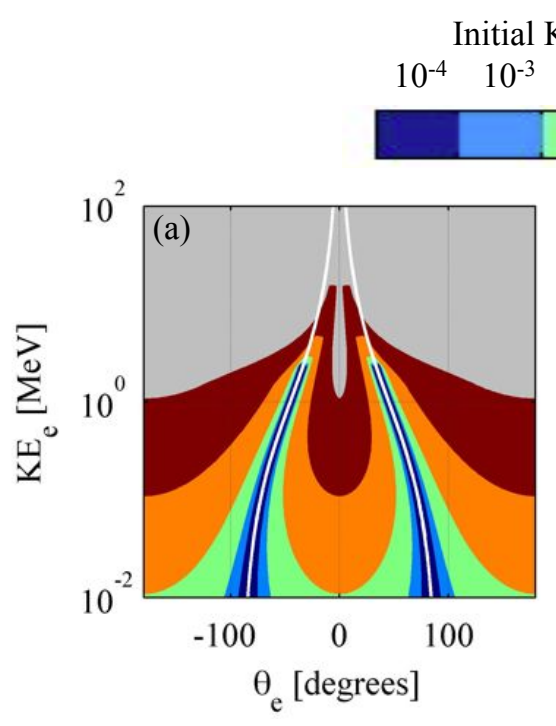

nitial $\mathrm{KE}_{\mathrm{e}}[\mathrm{MeV}]$

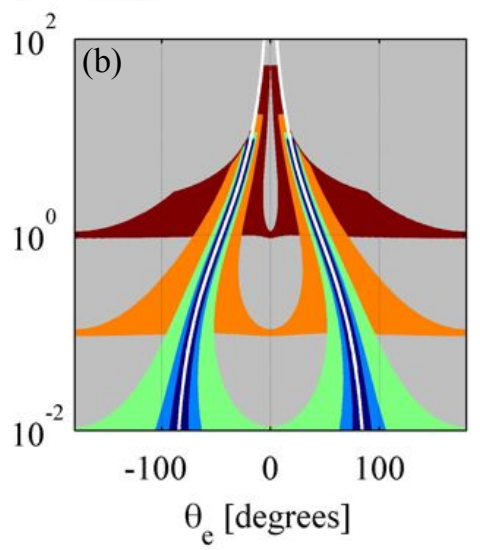

Figure 1.4: (Color) Hot-electron phase space calculations of test particles accelerated by plane-wave EM fields as a function of kinetic energy and angle (colored by initial kinetic energy). Electrons are injected at the (a) peak and (b) node locations of the electric field. The white curve indicates the classical ejection angle.

the maximum/minimum energy of the electron at any given time is strongly dependent on the phase at which it enters the field. From this simple calculation, electrons injected at the peak (Figure 1.4(a)) have the possibility of losing energy but electrons that are injected into the field at a node (Figure 1.4(b)) never have an instantaneous energy below that at which they were injected. ${ }^{3}$

In an experimental setting, real laser pulses, unlike plane-waves, have finite temporal and spatial profiles. Perhaps one of the most renowned examples of finite pulse phenomena is the ponderomotive force, which describes the tendency of a finite spatial laser profile to eject electrons from regions of high intensity to low. Starting from the Lorentz force equation (Equation 1.1) and substituting in the vector potential (i.e. $\vec{E}=-c^{-1} \partial \vec{A} / \partial t$ and $\vec{B}=\nabla \times \vec{A})$, we get

$$
\frac{\partial \vec{p}}{\partial t}+(\vec{v} \cdot \nabla) \vec{p}=\frac{e}{c} \frac{\partial \vec{A}}{\partial t}-\frac{\vec{v}}{c} \times(\nabla \times \vec{A})
$$

\footnotetext{
${ }^{3}$ While the phase electrons at which get injected into the fields isn't entirely controllable, conditions where free electrons are born into a field from field ionization, near the peak of the electric field strength, should be characteristic of Figure 1.4(a)
} 
and introducing the same normalizations of $\vec{\varrho}=\vec{p} / m_{e} c$ and $\vec{a}=e \vec{A} / m_{e} c^{2}$, this reduces to

$$
\frac{\partial \vec{\varrho}}{\partial t}+(\vec{v} \cdot \nabla) \vec{\varrho}=\frac{\partial \vec{a}}{\partial t}-\vec{v} \times(\nabla \times \vec{a})
$$

Using the vector identity $\nabla(\vec{\Lambda} \cdot \vec{\Gamma})=(\vec{\Lambda} \cdot \nabla) \vec{\Gamma}+(\vec{\Gamma} \cdot \nabla) \vec{\Lambda}+\vec{\Lambda} \times(\nabla \times \vec{\Gamma})+\vec{\Gamma} \times(\nabla \times \vec{\Lambda})$, this reduces to

$$
\begin{aligned}
\frac{\partial \underline{\varrho}}{\partial t}+\frac{c}{2 \gamma} \nabla \varrho^{2}-\vec{v} \times(\nabla \times \vec{\varrho}) & =\frac{\partial \vec{a}}{\partial t}-\vec{v} \times(\nabla \times \vec{a}) \\
\frac{\partial}{\partial t}(\varrho \vec{\varrho}-\vec{a}) & =-\frac{c}{2 \gamma} \nabla \varrho^{2}+\vec{v} \times(\nabla \times(\vec{\varrho}-\vec{a})) .
\end{aligned}
$$

For an electron starting with normalized momentum $\vec{\varrho}_{o}=\left(\varrho_{x_{o}}, \varrho_{y_{o}}, \varrho_{z_{o}}\right)$, we find from Equations 1.4 and 1.5 that $\vec{\varrho}-\vec{a} \propto \varrho_{\perp}^{2}(x) \hat{x}+\vec{\varrho}_{\perp_{o}}$ which is curl-less. Plugging this back into Equation 1.13 we find that the force acting on a electron will be

$$
\frac{\partial \vec{\varrho}}{\partial t}=\underbrace{\frac{\partial \vec{a}}{\partial t}}_{\text {fast }}-\underbrace{c \nabla \gamma}_{\text {slow }}
$$

where $\gamma=\sqrt{1+\varrho^{2}}$. The equation has two components: a fast oscillating component that varies like the fields vector potential, driving electron motion transverse to the laser propagation direction and a slow component that varies with spatial gradients in $\gamma$ (and hence intensity). This slow component expels electrons away from regions of high intensity and is often called the ponderomotive force.

Depending on how these electrons are injected into and de-phase from the plane-wave completely determines their final energy and trajectory characteristics after the interaction: control of both these aspects is critical to applications of interest in this thesis. With the exception of a few vacuum acceleration schemes [85], electrons in these experiments are typically born in and de-phased from the EM field by interacting with matter.

Most materials begin to ionize at laser intensities of $\sim 10^{11} \mathrm{~W} / \mathrm{cm}^{2}$ via multi-photon ionization, but over-the-barrier (OTB) ionization will quickly take over as soon as the field becomes strong enough to significantly perturb the Coulomb potential of the atom [85]. A schematic of OTB is shown in Figure 1.5, where the Coulomb potential $U_{C}$ of the nucleus (blue) is perturbed by a strong, homogeneous, external electric field $U_{E}$ (red), resulting in a 
barrier suppression $U_{B S}$ (black). Only an electric field $E$ with sufficient strength can perturb the potential well enough such that the atom can be ionized, i.e. where $U_{O T B}=U_{\text {ion }}$. The minimal barrier potential is found by solving $d U_{B S} / d x=0$, resulting in $x_{\text {OTB }}=\sqrt{Z e / E}$. For a given ionization potential $U_{i o n}$, the electric field potential must be $E=U_{\text {ion }}^{2} /\left(4 Z e^{3}\right)$, or more conveniently in terms of intensity

$$
I_{O T B}=\frac{c}{8 \pi} E^{2}=\frac{c U_{i o n}^{4}}{128 \pi Z^{2} e^{6}}
$$

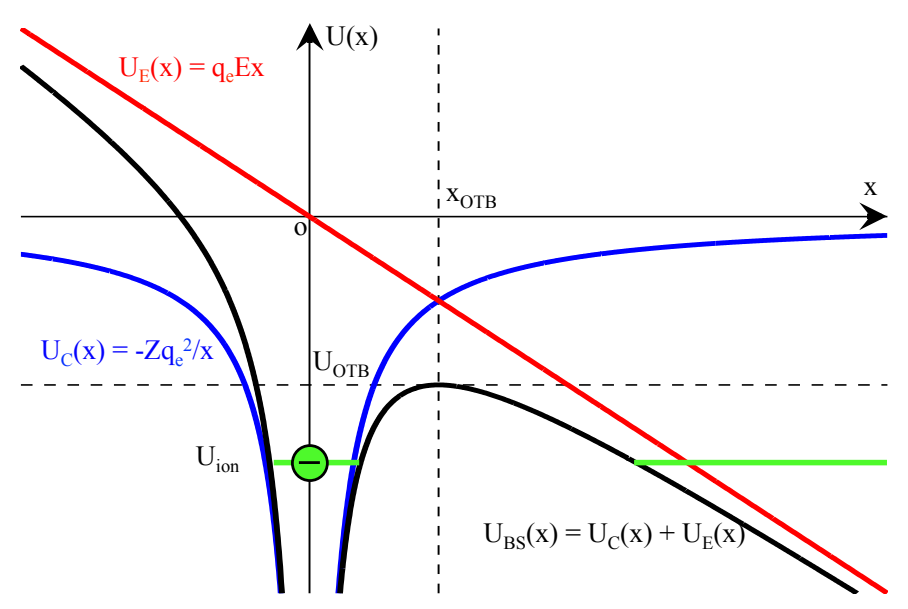

Figure 1.5: (Color) Schematic of over-the-barrier (OTB) field ionization. Energy of an electron in the Coulomb potential energy of the nucleus of an atom (blue) is perturbed by a strong, homogeneous external electric field (red), resulting in a barrier suppression (black).

Shown in Figure 1.6 are (a) the ionization potentials [86] of aluminum, copper and gold (some common materials to these types of experiments) along with (b) the corresponding laser intensities required to field ionize by sequential over-the-barrier (OTB) ionization. Tunneling ionization, when the electron wave-function can quantum mechanically 'leak' through the potential barrier can also occur at these intensities [85]; these rates were investigated by Ammosov, Delone and Krainov [87]. Depending on the rising edge of the 
laser pulse (particularly pre-pulse), drastically different pre-plasma environments can occur for different materials. This plasma formation not only provides a source of electrons for the laser to accelerate but also a means for them to de-phase from the alternating acceleration/deceleration cycles of the laser so a few fundamental laser-plasma phenomena are essential to understanding work in this thesis.
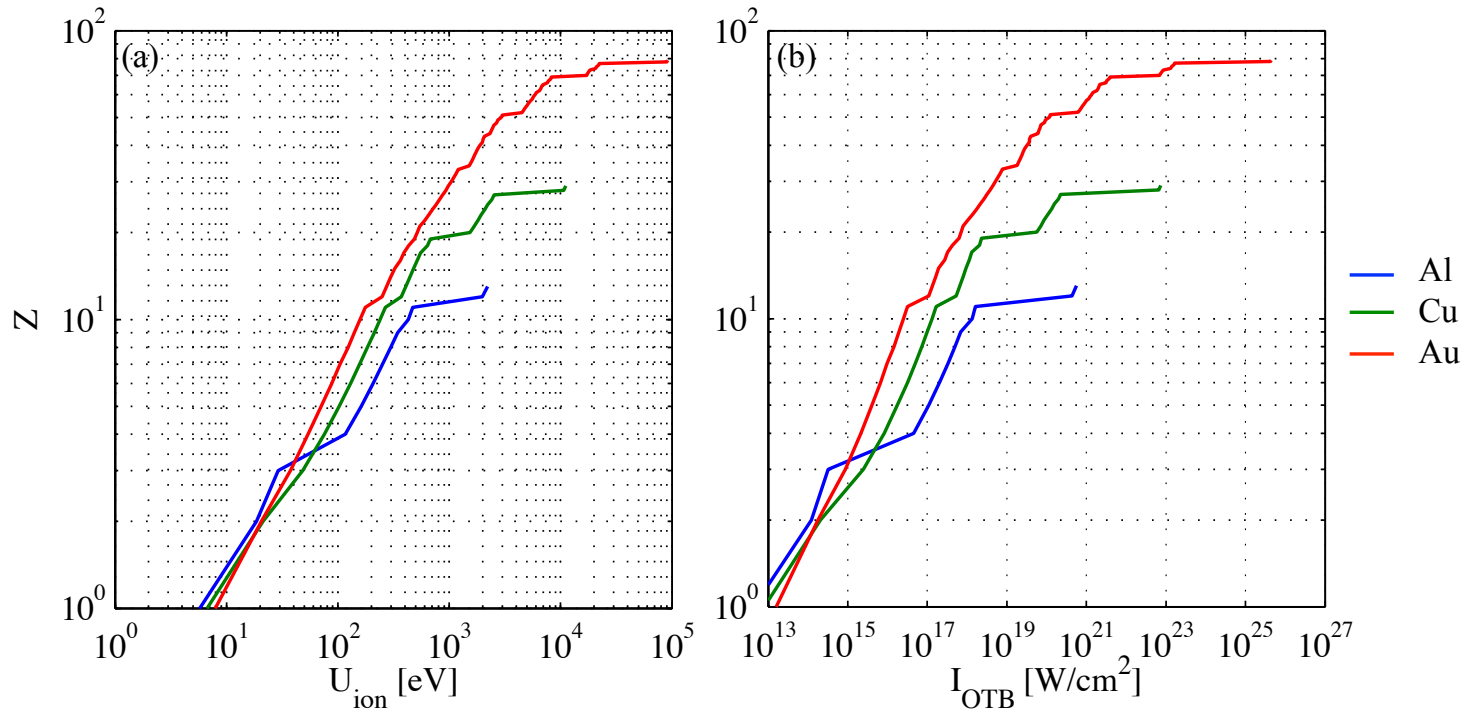

Figure 1.6: (Color) (a) Ionization potentials [86] and (b) corresponding field intensity for over-the-barrier ionization of aluminum ( $\mathrm{Al}$, blue), copper $(\mathrm{Cu}$, green) and gold $(\mathrm{Au}$, red) [85].

\subsubsection{Plasma fundamentals}

Plasma, in the most general sense, is an assembly of charged particles. The mobility of the particles results in a strong sensitivity to both internal fields generated from charges and currents, as well as external electric and magnetic fields. Electric and magnetic fields in 
this system obey Maxwell's equations, which in $c g s$ units are given by

$$
\begin{aligned}
\nabla \cdot \vec{E} & =4 \pi \rho \\
\nabla \cdot \vec{B} & =0 \\
\nabla \times \vec{E} & =-\frac{1}{c} \frac{\partial \vec{B}}{\partial t} \\
\nabla \times \vec{B} & =\frac{4 \pi}{c} \vec{j}+\frac{1}{c} \frac{\partial \vec{E}}{\partial t}
\end{aligned}
$$

where $\rho=n_{e}+\sum_{\alpha} Z_{\alpha} n_{\alpha}$ is the charge density $\left(\mathrm{cm}^{-3}\right)$ and $\vec{j}=e\left(-n_{e} \overrightarrow{v_{e}}+\sum_{\alpha} Z_{\alpha} \overrightarrow{v_{\alpha}}\right)$ is the current density (statampere $/ \mathrm{cm}^{2}$ ), both given as a sum on $\alpha$ over all ion species with charge $Z_{\alpha}$ and velocity $\vec{v}_{\alpha}$.

The attractive nature between charges of opposite sign often leads to macroscopically quasi-neutral plasmas (i.e. $\rho=0$ ), but thermal motion limits charge shielding on a microscopic level. The characteristic distance over which charges are screened in traditional plasmas is known as the Debye length $\lambda_{D}$, given by Equation 1.20 , where $k_{B}$ is the Boltzmann constant and $T_{e / \alpha}$ the electron/ion temperature [7].

$$
\lambda_{D}^{-2}=4 \pi e^{2}\left(\frac{n_{e}}{k_{B} T_{e}}+\sum_{\alpha} \frac{n_{\alpha} Z_{\alpha}^{2}}{k_{B} T_{\alpha}}\right)
$$

When ion motion is negligible, this equation reduces to the electron Debye length $\lambda_{D}=$ $\sqrt{k_{B} T_{e} /\left(4 \pi n_{e} e^{2}\right)}$. For a $1 \mathrm{keV}$, solid density, fully stripped aluminum plasma (typical of the reduced mass targets in these laser-plasma experiments), the Debye length is approximately $0.26 \mathrm{~nm}$ (nearly $2000 \times$ smaller than the laser wavelength at $527 \mathrm{~nm}$ ).

Plasma is also known to exhibit collective behaviors, such as the renowned electrostatic density oscillations. Consider an initially uniform quasi-neutral sheet of plasma which has been perturbed in such a manner that all the electrons $\left(n_{e} \approx Z n_{i}\right)$ have been displaced from their quasi-neutral positions a small distance $\delta x$ along the $x$-axis (fixed ions), illustrated in Figure 1.7. From Guass' law (Equation 1.16), we find the resulting electric field due to this charge separation to be

$$
\nabla \cdot \vec{E}=4 \pi Z n_{i} e \rightarrow \vec{E} \cdot \hat{x}=4 \pi n_{e} e \delta x
$$




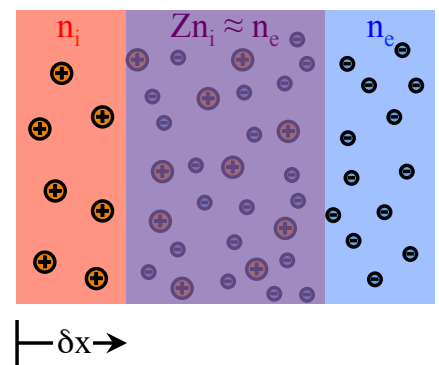

Figure 1.7: (Color) Illustration of plasma density oscillations. Electrons (blue) are displaced a distance $\delta x$ from the fixed ions (red), from the quasi-neutral (purple) region. The restoring electric field to neutralize the charge imbalance results in harmonic motion of electrons, oscillating at the plasma frequency $\omega_{p, e}$.

and the equation of motion for an electron in this field, due to this restoring field, is

$$
m_{e} \ddot{\delta_{x}}=-e E \rightarrow m_{e} \ddot{\delta x}+4 \pi n_{e} e^{2} \delta x=0
$$

which is simple harmonic motion where the angular frequency of the motion, commonly referred to as the electron plasma frequency, is given by

$$
\omega_{p, e}=\sqrt{\frac{4 \pi n_{e} e^{2}}{m_{e}}} .
$$

Since the plasma frequency is independent of wave-number $k=2 \pi / \lambda$ (i.e. dispersionless), the group velocity $v_{g}=\partial \omega_{p, e} / \partial k=0$ so plasma waves do not propagate energy. For our solid density, fully ionized aluminum plasma example above, the electron plasma frequency would be nearly $50 \mathrm{rad} / \mathrm{fs}$ (approximately $14 \times$ higher than the laser angular frequency at $3.57 \mathrm{rad} / \mathrm{fs})$.

While electron-electron and electron-ion collisions can lead to damping of plasma oscillations [88] (among other phenomena), the interaction region is rapidly heated to nearly collisionless conditions by the lasers of interest [89, 90]. Since collisions in the interaction region are minimal (the region of interest to this study), we will limit ourselves to only collisionless phenomena. 


\subsubsection{Electromagnetic waves in plasma}

Charged particles in plasma respond to external electromagnetic fields, but because of the mobility of electrons and their shielding tendencies, the fields begins to deviate from their vacuum form. For a non-relativistic linearly polarized plane-wave traveling along the $x$-axis (which for convenience we switch to the complex notation $\vec{E}=E_{o} e^{i\left(\omega_{L} t-k x\right)} \hat{y}$ ) traveling through a quasi-neutral, collisionless plasma, the electron motion is purely oscillatory in the electric field having quiver velocity $\overrightarrow{v_{e}}=i e /\left(m_{e} \omega_{L}\right) \vec{E} \hat{x}$ with corresponding current density $\vec{j}=-e n_{e} \overrightarrow{v_{e}}$. Taking the curl of Ampère's Law (Equation 1.24), we apply the vector identity $\nabla \times(\nabla \times \vec{B})=\nabla(\nabla \cdot \vec{B})-\nabla^{2} \vec{B}$ (where $\nabla \cdot \vec{B}=0$ ) and insert the oscillatory form of the electric field and current density to obtain Equations 1.25 and 1.26. Applying Faraday's Law (Equation 1.18), the equations reduce down to only a function of $\vec{B}$ as in Equation 1.27 .

$$
\begin{aligned}
\nabla \times(\nabla \times \vec{B}) & =\nabla \times\left(\frac{4 \pi}{c} \vec{j}+\frac{1}{c} \frac{\partial \vec{E}}{\partial t}\right) \\
-\nabla^{2} \vec{B} & =\nabla \times\left(-\frac{i 4 \pi n_{e} e^{2}}{m_{e} \omega_{L} c} \vec{E}+\frac{i \omega_{L}}{c} \vec{E}\right) \\
& =\frac{i \omega_{L}}{c}\left(-\frac{\omega_{p, e}^{2}}{\omega_{L}^{2}}+1\right) \nabla \times \vec{E} \\
\rightarrow-\nabla^{2} \vec{B} & =\frac{i \omega_{L}}{c^{2}}\left(\frac{\omega_{p, e}^{2}}{\omega_{L}^{2}}-1\right) \frac{\partial \vec{B}}{\partial t}
\end{aligned}
$$

Since $\vec{B}$ is also periodic in $x$ and $t$, we simply Fourier transform (i.e. where $\nabla \rightarrow-i k$ and $\left.\partial / \partial t \rightarrow i \omega_{L}\right)$ and find that the expression reduces to the plasma dispersion relation (Equation 1.28) [91].

$$
c^{2} k^{2}=\omega_{L}^{2}-\omega_{p, e}^{2}
$$

From the dispersion relation, we find that electromagnetic waves with $\omega_{L}<\omega_{p, e}, k$ becomes imaginary and the fields exponentially decays with distance. The electron density at which $\omega_{L}=\omega_{p, e}$ is called the critical density $n_{c}=m_{e} \omega_{L}^{2} /\left(4 \pi n_{e} e^{2}\right)$ as it is the highest density plasma which can support propagation of the electromagnetic fields. Beyond this density, electrons effectively shield the plane-wave's electric field since $\omega_{p, e}>\omega_{L}$. For the 
$527 \mathrm{~nm}$ laser used in this experiment, this corresponds to an electron density of $4.03 \times$ $10^{21} \mathrm{~cm}^{-3}$, nearly $45 \times$ lower than a cold, solid density aluminum target.

The index of refraction of the plasma is given by $\eta=\sqrt{1-\omega_{p, e}^{2} / \omega_{L}^{2}}$ which is always less than or equal to unity. The phase velocity of the wave, $v_{\phi}=\omega_{L} / k=c / \eta$, blows up to infinity while the group velocity, $v_{g}=\partial \omega_{L} / \partial k=c \eta$ goes to zero at the critical density since $\eta=0$. At densities above the critical density, the field strength decays exponentially, falling off by an e-folding with with a characteristic skin depth $l_{s}=k^{-1}$ or

$$
l_{s}=\frac{c}{\omega_{L} \sqrt{1-\frac{\omega_{p, e}^{2}}{\omega_{L}^{2}}}} .
$$

Since this evanescent wave doesn't propagate any energy, the electromagnetic energy that is not absorbed by the target is reflected. Electrons, uninhibited by this critical density electromagnetic cutoff, can now dephase from the oscillating accelerations and decelerations of the electromagnetic field, entering the supra-critical density target with net momentum and a trajectory characteristic of its acceleration process.

\subsubsection{Non-linear and relativistic laser-plasma phenomena}

All of the work in Chapter 1.5.3 assumed non-relativistic field, but the pulses of interest are clearly relativistic with $a_{o}>1$. Electrons in these relativistic fields begin to quiver in the electric field with velocities approaching the speed of light. These relativistic electrons effectively have their mass increased by the cycle averaged Lorentz factor $\gamma=\sqrt{1+a_{o}^{2} / 2}$ (i.e. $\left.m_{e} \rightarrow \gamma m_{e}\right)$. Along with the finite temporal and spatial envelopes of a real pulse, relativistic effects can significantly alter the spectral, temporal and spatial distributions of the incident pulse in physically intriguing, although often devastating, ways as the pulse propagates through under-dense plasma. Generally speaking, any phenomena that can modify the local plasma frequency, either in space or time, can induce pulse altering phenomena and instabilities. This can only be accomplished in two ways: (1) modify the electron density or (2) relativistic effects. 


\section{Spectral modification}

Apart from spectral dispersion, ${ }^{4}$ a changing electron density introduces an additional phase accumulation. The amount of phase $\phi$ accumulated by the pulse by propagating a distance $L$ through a spatially and temporally varying electron density (with index of refraction $\eta$ ) is given by Equation 1.30 [92].

$$
\phi=\frac{\omega_{L}}{c} \int_{x_{o}}^{x_{o}+L} \eta\left(x^{\prime}, t\right) d x^{\prime}
$$

The corresponding change to the instantaneous wavelength is then simply $\omega_{\text {inst }}=\omega_{L}-$ $\partial \phi / \partial t$. Modifications to the local electron density can come about by either ionization $\left(\dot{n}>0 \rightarrow \dot{\eta}<0 \rightarrow \dot{\phi}<0 \rightarrow \omega_{\text {inst }}>\omega_{L}\right.$, blueshift $)$ or recombination $(\dot{n}<0 \rightarrow \dot{\eta}>0 \rightarrow \dot{\phi}>$ $0 \rightarrow \omega_{\text {inst }}<\omega_{L}$, redshift). Considering only OTB field ionization, ${ }^{5}$ the charge state of the aluminum targets shot in the experiment (peak intensities of $\sim 4 \times 10^{19} \mathrm{~W} / \mathrm{cm}^{2}$ ) saturates at $Z=11$ which can be reached at $\gtrsim 1.6 \times 10^{18} \mathrm{~W} / \mathrm{cm}^{2}$. Given the temporal profile of the pulse (and lack of recombination), field ionization should only produce a blueshift in the first several $10 s$ of femtoseconds of the rising edge of the pulse for our aluminum targets. While ionization will saturate for this pulse with aluminum, other materials, like gold for example, will continue to be influenced by ionization effects up to the peak of the pulse. For a plasma that starts with a single charge state (i.e $\mathrm{Z}$ starts the same everywhere), one might also expect this blueshift to come in discrete intervals because of the discreteness of the ionization intensity curve (Figure 1.6), although they are not likely to be observed with the limited temporal resolution of current measurement techniques. This trend of field ionization induced spectral blueshifting $\left(\Delta \omega / \omega_{L}=\left(\omega_{\text {inst }}-\omega_{L}\right) / \omega_{L}\right.$, green $)$ is illustrated in Figure 1.8 along with the incident pulse intensity (black).

The laser pulse can also gain spectral shifts from the motion of the reflection surface

${ }^{4}$ Although not addressed in this study, dispersion can be an issue for extremely short pulses with a 'broad' spectrum.

${ }^{5}$ Although not addressed in this study, other means for changes in electron density than field ionization can come from collisional ionization/recombination [93]. 


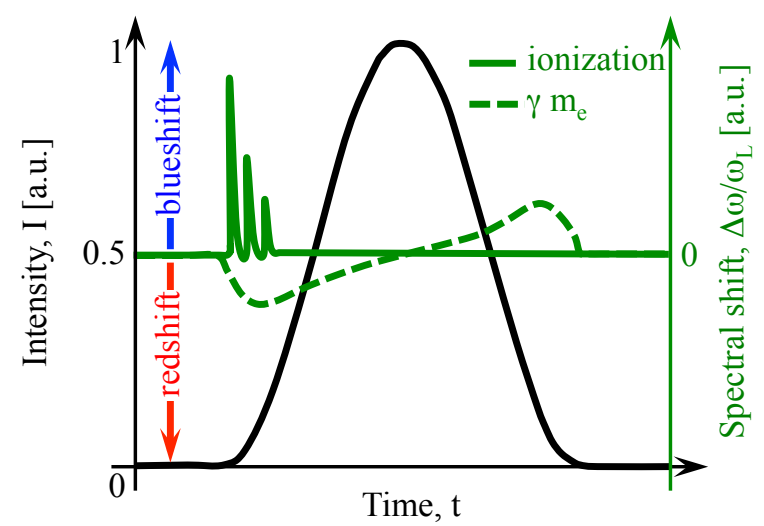

Figure 1.8: (Color) Spectral modifications to electromagnetic pulses (intensity shown in black) propagating through under-dense plasma due to ionization (solid green) and relativistic mass effects (dashed green). Ionization phenomena induce discrete blueshifts, saturating when the ions are fully stripped, while relativistic effects create alternating early red then late blueshifts about the peak of the pulse.

(i.e. the location of the critical density). The Doppler shift of the reflected light is given by

$$
\begin{aligned}
& \frac{\Delta \omega}{\omega_{L}}=-\frac{2 \beta_{c}}{1+\beta_{c}} \\
& \frac{\Delta \lambda}{\lambda_{L}}=\frac{2 \beta_{c}}{1-\beta_{c}}
\end{aligned}
$$

where $\beta_{c}=v_{c} / c$ is the motion of the reflecting surface described by the relativistic critical density contour (normalized to the speed of light). A receding surface $\left(v_{c}>0\right)$ results in a redshift and an expanding surface $\left(v_{c}<0\right)$ a blueshift. Since field ionization can only increase the local electron density, the critical surface expands away from the original solid density interface creating an additional blueshift upon reflection which behaves similarly to the ionization induced shifts discussed previously (green curve in Figure 1.8). Recombination, thermal plasma expansion and radiation pressure can also cause motion of the critical surface which, depending on their relative strengths, can lead to either a red or blueshift.

Once the pulse becomes relativistic (i.e. $a \gtrsim 1$ ), additional shifting mechanisms become available through the relativistic mass effect, i.e. $m_{e} \rightarrow \gamma(t) m_{e}$ where $\gamma(t)=\sqrt{1+a_{o}^{2}(t) / 2}$. This modified electron mass allows the laser to propagate to densities higher than the 
critical density $\left(n_{c} \rightarrow \gamma n_{c}\right)$, what is commonly referred to as self-induced transparency $[48,94]$. Since the relativistic Lorentz factor varies in time, the location of the relativistic critical surface changes throughout the duration of the pulse. On the rising edge of the pulse $\dot{\gamma}>0$, resulting in $v_{c}>0$ and a corresponding Doppler induced redshift upon reflection. On the trailing edge of the pulse, after the peak has reflected, the reverse holds true with a blueshift. For a pulse that is symmetric in time and pre-plasma that is not dynamically changing, this produces a symmetric spectral broadening about the peak of the pulse (i.e. $\Delta \omega / \omega_{L}$ is an odd function). This is illustrated in Figure 1.8 by the dashed green line.

Akin to the ionization induced spectral shift in the laser as it propagates through the under-dense plasma, the relativistic mass effect can also lead to additional phase, commonly referred to as self-phase modulation [42]. On the rising edge of the pulse, $\dot{\gamma}>0$, resulting in $\dot{\eta}<0$ with a corresponding redshift. On the trailing edge of the pulse, after the peak, the reverse holds true with a blueshift. Since self-phase modulation also depends on the sign of $\dot{\gamma}(t)$, the corresponding spectral shifting trends look similar to self-induced transparency (again, dashed green line in Figure 1.8).

\section{Temporal/spatial envelope modifications}

Since the pulse has finite temporal and spatial profiles (i.e. not a plane-wave), the relativistic Lorentz factor will vary in both time and space. Since $\eta(t)$ also varies with $\gamma(t)$, the group velocity $\left(v_{g}=\eta c\right)$ of the pulse will also vary in time. The peak of the pulse has a higher $\gamma$ than the wings, and with a higher index of refraction. In under-dense plasma, the peak of the pulse can travel faster than the leading or trailing edges, resulting in temporal pulse front sharpening [43], illustrated in Figure 1.9(a). The difference in the time required for a relativistic pulse of to travel to the critical density (indicated by $x_{c}$ ) is given by Equation 1.33: negative values of $\Delta t$, like those for $\gamma>1$ for relativistic intensities, implies that

the front of the pulse would sharpen and conversely, positive values would imply that the trailing edge of the pulse would sharpen.

$$
\Delta t(I)=\int_{0}^{x_{c}}\left[\frac{1}{v_{g}(x, \gamma(I))}-\frac{1}{v_{g}(x, \gamma=1)}\right] d x
$$




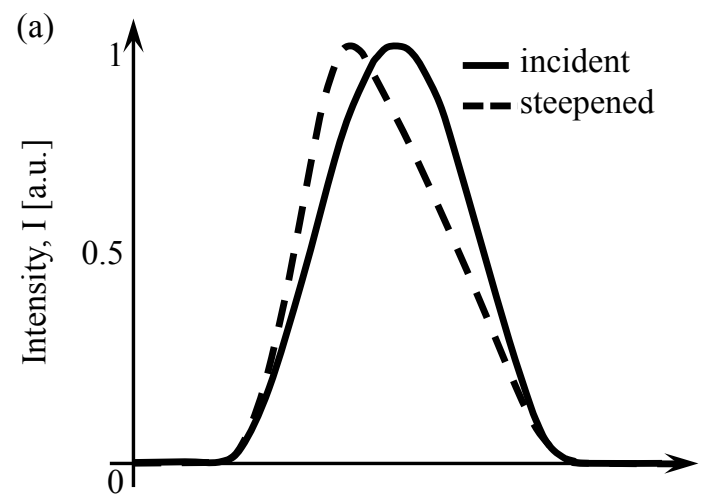

Time, $\mathrm{t}$

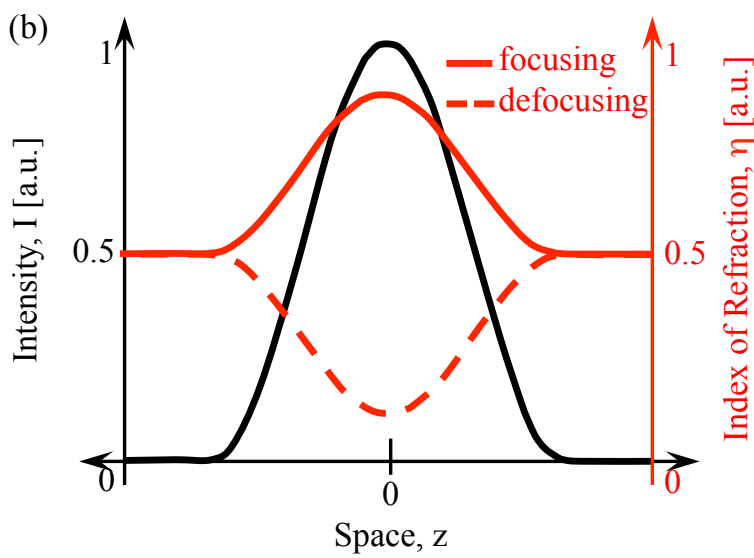

Figure 1.9: (Color) (a) Illustration of temporal profile steepening and (b) focusing/defocusing of a relativistic laser pulse propagating in under-dense plasma.

Modifications to the spatial envelope of the incident laser pulse as it propagates through the under-dense plasma can come from spatial gradients in the index of refraction (transverse to the laser propagation direction). From Snell's Law, an index of refraction that is concave-down $\left(\eta^{\prime \prime}(x)<0\right.$, solid red) will result in focusing while one that is concave-up $\left(\eta^{\prime \prime}(x)>0\right.$, dashed red) causes defocusing, shown in Figure 1.9(b) along with the incident spatial laser intensity profile (black). The relativistic mass correction increases the index of refraction for higher intensity portions of the pulse (concave-down), leading to what is known as relativistic self-focusing [95]. Another source of focusing can occur when the electron population is decreased (from recombination) or evacuated (by direct laser acceleration and the ponderomotive force) from the laser focal region. Alternatively, defocusing (concave) can occur when the electron density at the peak intensities is higher than in the wings, such as by ionization.

Relativistic self-focusing increases the intensity of the pulse, feeding into the self-focusing mechanism which continues to focus the beam down until balanced by the self-diffraction of the laser pulse, resulting in the filamentation instability [96]. For a pulse with a Gaussian 
spatial envelope, $a(z)=a_{o} \exp \left(-z^{2} / w_{o}^{2}\right)$, the condition for which this occurs is given by [85]

$$
\frac{a_{o} \omega_{p, e} w_{o}}{c} \geq 4
$$

where $w_{o}$ is the $1 / e$ falloff of the vector potential amplitude, often referred to as the beam waist. For a Gaussian beam with $a_{o}=3, \lambda_{L}=0.527 \mu m$ and $w_{o}=5 \mu m$, the electron density at which this occurs is $\approx 2.01 \times 10^{18} \mathrm{~cm}^{-3}$, approximately $2000 \times$ smaller than the critical density. Any phenomena that can create transverse index modulations can seed this instability, often breaking the pulse up into many smaller filaments.

Other spatial/temporal envelope modifications can arise from non-uniform absorption of laser energy into the target. Absorption is highly non-linear as collisional processes are important in the non-relativistic portions of the pulse $\left(a_{o}<1\right)$ while collisionless mechanisms dominate the relativistic portions $\left(a_{o} \gtrsim 1\right)[90]$.

\subsubsection{Laser-driven quasi-static magnetic field generation}

The interaction between a relativistic laser pulse and under-dense plasma can drastically change the spectral/temporal and spatial properties of the accelerating electromagnetic fields which directly relates to how electrons gain energy. Aside from the aforementioned laser-plasma instabilities, quasi-static magnetic fields can develop in the laser interaction region reaching hundreds of MegaGauss over micron spatial scales. In some circumstances, these quasi-static magnetic fields have magnitude and extent that can significantly affect (if not completely trap) even relativistic electron trajectories and have reasonably been assumed to be responsible for more randomized accelerated electron trajectories in relativistic laser-plasma interactions [72, 89].

To determine the trapping conditions, we consider a uniform magnetic field with strength $B$ with an electron traveling perpendicular to the magnetic field orientation with velocity $v_{e}$. The magnetic field can do no work since the force on the electron is perpendicular to the motion, driving the electron to move in a circle. The radius of this circle is given by 
the gyroradius $r_{g}=v_{e} / \omega_{c}$ where $\omega_{c, e}$ is the electron cyclotron frequency

$$
\omega_{c, e}=\frac{e B}{\gamma m_{e} c}
$$

with $\gamma=\sqrt{1+p_{e}^{2} /\left(m_{e}^{2} c^{4}\right)}$. The gyroradii of an electron in a 125, 250, and $500 M G$ magnetic fields (blue, green and red respectivley), typical values for some of these phenomena, are shown in Figure 1.10 as a function of electron energy. Any magnetic field with a spatial extent $\geq 2 \times r_{g}$ will be able to trap electrons: for example, an electron with $K E_{e}=1 \mathrm{MeV}$ will be completely trapped by a $500 M G$ magnetic field if it is $\gtrsim 200 \mathrm{~nm}$.

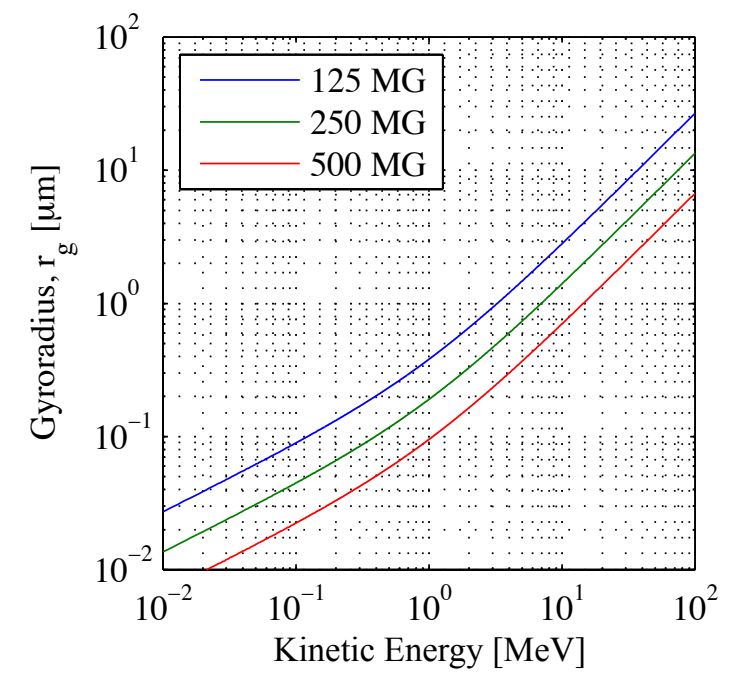

Figure 1.10: (Color) Gyroradius of electrons in external uniform magnetic fields.

Magnetic field generation at sub and supra-critical densities can come from many sources, all of which rely on generating currents. The thermoelectric effect [45] arises when the electron temperature gradient is not parallel to the electron electron density gradient, i.e. $\nabla T_{e} \times \nabla n_{e} \neq 0$. These fields typically develop over hydrodynamic time scales $(\sim$ picoseconds), reaching $\sim 10 s$ of $M G$ and typically dominate the magnetic fields outside the laser focal region. Another source of MegaGauss magnetic fields arises from 
the ponderomotive force of the laser [46]. As discussed previously, the ponderomotive force of of the laser expels electrons away from the laser focal region, driving a DC current and with it a magnetic field which scales like $\nabla n_{e} \times \nabla I_{o}$. Since this mechanism relies on the ponderomotive force, it develops close to the laser focal region over laser cycle timescales but with the opposite sign of the thermoelectric effect. For relativistic laser pulses $\left(a_{o} \gg 1\right)$, this mechanism has been predicted to create hundreds of $M G$ magnetic fields which are on-par with the incident laser fields [46]. These phenomena are illustrated in Figure 1.11, where the electron density (shaded gray area) gradient $\nabla n_{e}$ points up and electron temperature and laser (green) intensity gradients $\left(\nabla T_{e}\right.$ and $\nabla I$ respectively) point radially inward. The signs of the thermoelectric effect is shown in blue (where the curve indicates strength) and the ponderomotive effect is shown in red. Since both mechanisms rely on the electron density gradient, these fields are typically strongest near the critical surface.

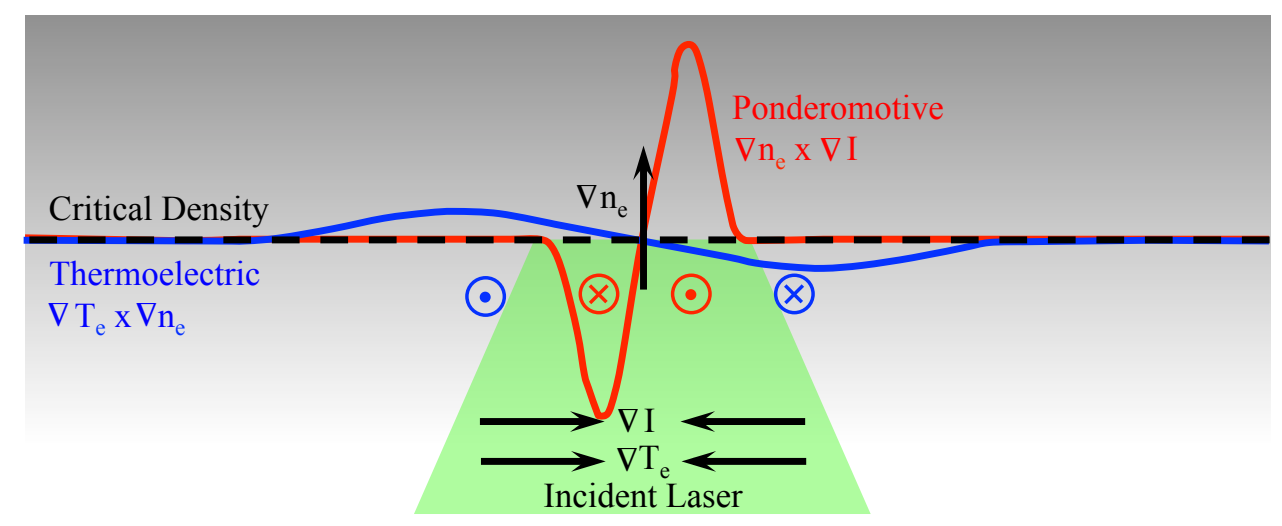

Figure 1.11: (Color) Illustration of thermoelectric (blue) and the ponderomotive (red) sources of quasi-static magnetic field generation.

Hot-electrons generated in the relativistic LPI can also create significant currents [44]. For collisional plasmas, a resistive cold return current will eventually develop which neutralizes the hot-electron current [97], but of more interest here is what happens in the collisionless laser-plasma regime within a few skin depths of the laser interaction region but 
still at supra-critical densities. The electron distribution in this region is highly anisotropic, consisting of both highly directional laser driven hot-electrons and, more-or-less, an omnidirectional cold neutralizing electron current [98]. This results in an electromagnetic form of the two-stream instability, often referred to as the Weibel instability [99], which causes the hot-electron currents to filament and create several $100 M G$ magnetic filaments that extend over sub-wavelength spatial scales inside solid density.

\subsubsection{Over-dense interactions}

By switching to high-contrast pulses, we are attempting to avoid the pitfalls of the laserplasma interactions described in the previous sections. High-contrast pulses, however, have the additional complication associated with getting the electrons from sharp, supra-critical density target interfaces into the relativistic fields.

In ultra-intense interactions, absorption is dominated by collisionless processes [85, 90] and several heating mechanisms have been suggested. Vacuum heating, often referred to as the Brunel mechanism [100], relies on a component of the laser electric field being normal to the interface that can push and pull electrons from within a few skin depths across the vacuum interface but is only present for p-polarized lasers at non-normal incidences. Alternatively, relativistic $\vec{j} \times \vec{B}$ heating [101] can exist at normal incidence as it relies on the oscillating $\vec{v} / c \times \vec{B}$ component of the Lorentz force within a few skin depths to drive electrons across the interface, which will work for any arbitrary linear polarization. In both of these mechanisms (illustrated in Figure 1.12(a)), electrons within a few skin depths are pushed and pulled away from the supra-critical interface, creating a charge imbalance. A sheath field $\vec{E}_{d}$ develops to neutralize the imbalance, pulling electrons into vacuum before turning them around and accelerating them to the point where they can dephase at the solid density interface and escape beyond a few skin depths. The work done on the electron in either scenario is by this normal sheath field.

For perfectly flat interfaces irradiated at normal incidence, low coupling results in a standing-wave (SW) field pattern near the interface from the interference between the incident and reflected waves. Electrons that eventually reach vacuum are accelerated with 

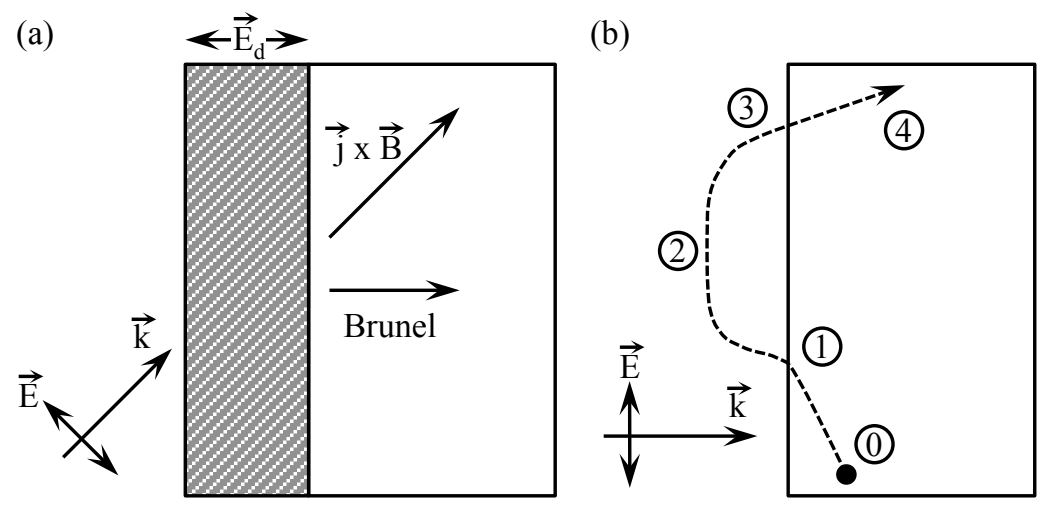
$0 . \mathrm{e}^{-}$begins with large transverse momentum
1. interface B turns $\mathrm{e}^{-}$toward vacuum
2. $\mathrm{e}^{-}$accelerated to $\sim 2 \mathrm{a}_{\mathrm{o}}$ by $\mathrm{E}$ peaked at $\lambda_{\mathrm{L}} / 4$ from interface
3. increasing B turns $\mathrm{e}^{-}$ toward solid density 4. $\mathrm{e}^{-}$dephases, escaping into solid density

Figure 1.12: (Color) Illustration of traditional electron acceleration mechanisms in the presence of sharp, supra-critical density plasma interfaces free of under-dense plasma. Brunel and relativistic $\vec{j} \times \vec{B}$ rely on the electrostatic sheath (shaded gray area) formed by charge imbalance at the interface to do work on the electron while work in (b) the $2 a_{o}$ mechanism is done by the laser's electric field which is parallel to the interface.

trajectory characteristics unique to this standing-wave structure, referred to as the $2 a_{o}$ mechanism (illustrated in Figure 1.12(b)). For the perfect conductor discussed by May et al [102], only electrons inside the plasma with sufficient transverse momentum (approaching $m_{e} c$ ) are able to reach vacuum because of the strong surface magnetic fields. Once in vacuum, electrons are quickly accelerated to $\varrho \sim 2 a_{o}$ by the electric field parallel to the surface (peaked at $\lambda_{L} / 4$ away from the interface) and then turned back toward solid density by the growing magnetic field. This acceleration mechanism differs from both Brunel and relativistic $\vec{j} \times \vec{B}$ heating in that no electric field component normal to the surface is required and all the work is done by the lasers electric field, parallel to the surface. However, it is not present in plasmas with electron energies below a few $10 \mathrm{~s}$ of $\mathrm{keV}$ and does not address how initially cold targets can reach this state. 


\subsection{Simulation fundamentals}

\subsubsection{Introduction}

State-of-the-art simulations used to study these laser-plasma interactions require a variety of algorithms to handle the sweeping range of plasma densities (from vacuum to solid density), electromagnetic wave propagation and its interaction with matter, transport and generation of radiation and charged particles in solid density plasmas, equation-of-state of warm-dense matter, et cetera. Additionally, the interactions that we desire to model occur over a vast range of spatial and temporal scales with on the order of an Avagadro's number of electrons and ions; we desire to model macroscopic laser-plasma interactions, occurring over $\sim 100 \times 100 \times 100 \mu m^{3}, \sim 10$ picoseconds, which are sensitive to microscopic physics phenomena requiring $\sim$ nanometers, $\sim$ attoseconds resolutions.

Despite significant computational advances over the past several decades, state-of-theart simulations are still incapable of handling this scale of interaction without any simplifications. That being said, models have been developed to capture the essence of the plasma response with a manageable size simulation, but often with the loss of some level of finer, although often irrelevant, detail. More often than not, a kinetic description of the plasma and charged particles is adopted for these relativistic interactions where a large number of charged particles in the laboratory setting are represented by a single 'macroparticle' which has the same charge to mass ratio $(q / m)$ as the component charges so that the equations of motion are unchanged.

The positions and momenta of these macroparticles are self-consistently advanced using the Lorentz force (Equation 1.1) and Maxwell's equations (Equation 1.16), where the equations are discretized into a finite-difference form for numerical integration. Field quantities are calculated at discrete grid locations by interpolating macroparticle charge and current density in each cell to the grid points. This is commonly referred to as particle-in-cell (PIC) modeling. In these simulations, the grid mesh was fixed (often referred to as an Eulerian grid) but other algorithms employ an adaptive, or Lagrangian, mesh. Kinetic simulations, however, tend to create noisy fields because of the discreteness of the macroparticles. If 
chosen wisely, charges with finite size, described by a particle shape or 'cloud,' can remove the self-force of a own particle's field on itself and smooth over high frequency noise, often referred to as cloud-in-cell (CIC) [103].

The spatial $\Delta x$ and temporal $\Delta t$ resolution required by the simulation are constrained by the physics of interest and the integration algorithms of choice. To resolve the wave propagation of the laser, we would require $\Delta x \ll \lambda$ and $\Delta t \ll \Delta x / c$ (known as the Courant limit) for numerical stability. For plasma, we would ideally want to resolve the Debye length (i.e. $\left.\Delta x \ll \lambda_{D}\right)$ for charge screening phenomena and the electron plasma frequency (i.e. $\left.\Delta t \ll 2 \pi / \omega_{p, e}\right)$ for plasma wave oscillations. In the under-dense regions of the grid, the laser resolution requirements are typically more strict than the plasma requirements but the reverse can hold true at supra-critical densities.

Two numerical approaches exist for solving these coupled partial differential equations: explicit and implicit integration algorithms. Explicit integration is the most straight-forward approach, relying only on previous values to determine current conditions. As such, both the Debye length and the plasma frequency must be fully resolved for numerical stability (often creating very large and computationally expensive simulations). In implicit algorithms, both previous and estimated future quantities are used to calculate the current quantity of interest. Although being more computationally expensive than explicit algorithms per time step, implicit algorithms can offer to relaxed temporal resolution constraints for numerical stability, mocking up a plasma-like response for longer interactions while strongly damping under-resolved high-frequency phenomena. All of the simulations in this thesis were performed with the direct implicit algorithm implemented in the commercially available electromagnetic PIC code Large Scale Plasma (LsP) [104].

\subsubsection{Direct implicit algorithm}

In the direct implicit algorithm in LsP, outlined in Figure 1.13, particles receive two halfpushes for every time step, separating the influence of past and future fields on particle motion: the first half-push arises from previous field values and then the second from 
predicted fields. ${ }^{6}$ Closely following Welch $[104,105]$, the algorithm begins with the Lorentz force acting on a electron in an electric $\vec{E}$ and magnetic $\vec{B}$ field, which in finite-difference form looks like

$$
\frac{\vec{p}_{n+1 / 2}-\vec{p}_{n-1 / 2}}{\Delta t}=\vec{a}_{n}+\frac{\vec{p}_{n+1 / 2}+\vec{p}_{n-1 / 2}}{2} \times \frac{q}{\gamma_{n} m c} \vec{B}_{n}\left(\vec{x}_{n}\right)
$$

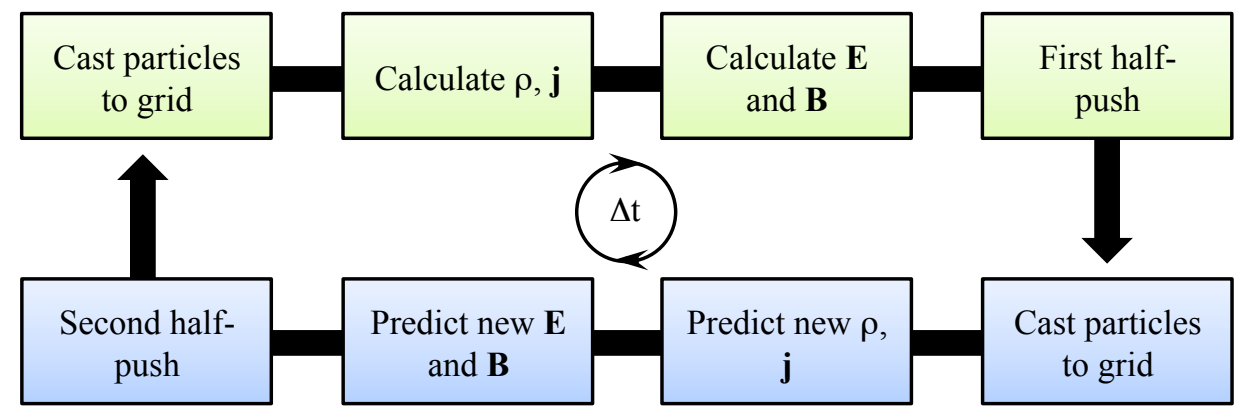

Figure 1.13: (Color) Outline of one time step $\Delta t$ in the direct-implicit algorithm implemented in LsP. Each time step, particles receive two half-pushes; the first half-push is from the previous field values and the second from future ones.

where $\vec{p}_{n}$ is the charge's momentum at time step $n$ and $\vec{a}_{n}=1 / 2\left[\left(\vec{a}_{n-1}+(q / m) \vec{E}_{n+1}\left(\vec{x}_{n+1}\right)\right)\right]$ is the average acceleration from old and new electric field values. The relativistic Lorentz factor $\gamma_{n}$ is calculated using $1 / 2 \vec{a}_{n-1}$ (the first half-push) and field quantities are interpolated from the grid to the particle position. Each particle's position and velocity aren't simultaneously known; i.e. the particle position is known at integer time steps $\left(\vec{x}_{n \pm 1}\right)$ and velocity at integer-and-a-half time steps $\left(\vec{v}_{n \pm 1 / 2}\right)$ according to

$$
\begin{aligned}
\vec{v}_{n+1 / 2} & =\vec{v}_{n-1 / 2}+\Delta t \vec{a}_{n} \\
\vec{x}_{n+1} & =\vec{x}_{n}+\Delta t \vec{v}_{n+1 / 2}
\end{aligned}
$$

in what is known as a 'leapfrog' configuration [103].

The equation of motion is solved by first linearizing the equation into the form $\vec{p}_{n+1 / 2}=$

${ }^{6}$ Sub-grid models, like ionization, collisions and nuclear reactions, are implemented after the first halfpush. 
$\underline{\underline{T}} \vec{A}$ where $\underline{\underline{T}}$ is the magnetic field rotation tensor

$$
\underline{\underline{T}}=\frac{1}{1+\Omega^{2}}\left[\begin{array}{ccc}
1+\Omega_{1}^{2} & \Omega_{1} \Omega_{2}+\Omega_{3} & \Omega_{1} \Omega_{3}-\Omega_{2} \\
\Omega_{1} \Omega_{2}-\Omega_{3} & 1+\Omega_{2}^{2} & \Omega_{2} \Omega_{3}+\Omega_{1} \\
\Omega_{1} \Omega_{3}+\Omega_{2} & \Omega_{2} \Omega_{3}-\Omega_{1} & 1+\Omega_{3}^{2}
\end{array}\right]
$$

where $\vec{\Omega}=\Delta t q \vec{B}_{n} /(2 \gamma m c)$ and $\vec{A}=\vec{p}_{n-1 / 2}+\Delta t \vec{a}_{n}+\vec{p}_{n-1 / 2} \times \vec{\Omega}$. For the two half-pushes, $\vec{A}$ is split into two components: $\vec{A}=\vec{A}_{1}+\vec{A}_{2}$ where $\vec{A}_{1}=\vec{p}_{n-1 / 2}+1 / 2 \Delta t \vec{a}_{n-1}+\vec{p}_{n-1 / 2} \times \vec{\Omega}$ is the first half-push with the old field values and $\vec{A}_{2}=(q / 2 m) \vec{E}_{n+1}\left(\vec{x}_{n+1}\right)$ is the second with the predicted fields.

In order to predict the new fields, the additional current that is missing from the second half-push acceleration, $\delta \vec{J}$, needs to be calculated. In LsP, the estimated current density takes the form $\vec{J}=\vec{J}^{1}+\delta \vec{J}$ where $\vec{J}^{1}$ is the current density from the first half-push and $\delta \vec{J}=\underline{\underline{S}} \cdot \vec{E}_{n+1}\left(\vec{x}_{n+1}\right)$ is the perturbed current from the second half-push. Due to its similar form to an electric susceptibility [105], $\underline{\underline{S}}$ is called the implicit susceptibility tensor given by

$$
\underline{\underline{S}}=\frac{\rho \Delta t q}{2 \gamma_{n+1 / 2} m}\left(\underline{\underline{T}}-\vec{v}_{n+1 / 2} \otimes \vec{v}_{n+1 / 2}\right)
$$

where $\underline{\underline{S}}$ is is scattered to the grid after the first half-push and the future fields are then calculated by

$$
\begin{aligned}
& \frac{\partial \vec{B}}{\partial t}=-\nabla \times \vec{E} \\
& \frac{\partial \vec{E}}{\partial t}=\nabla \times \vec{B}-\vec{J}^{1}-\underline{\underline{S}} \cdot \vec{E}_{n+1}\left(\vec{x}_{n+1}\right) .
\end{aligned}
$$

The implicit susceptibility tensor is what allows future fields to be estimated using old quantities. This system of equations governing the predicted electric and magnetic fields, once discretized into a finite-difference format, can be solved either iteratively (using the Alternating Direct Implicit (ADI) method) or with matrix inversion algorithms. Since the susceptibility tensor only provides an estimate of the missing current density (i.e. $\delta \vec{J}$ doesn't exactly conserve charge), the difference between the final current and the two-step based current estimate is gradually incorporated back into the simulation over a specified number 
time steps [104].

\subsubsection{Implementation}

The direct implicit algorithm implemented in LSP relaxes the the constraint on the time step $\Delta t$ to the extent that $\omega_{p, e}$ and $\omega_{c, e}$ do not need to be simultaneously resolved, although both cannot be under-resolved at the same time in the same place [105]. This allows us to model a plasma-like material response with greater numerical stability for longer time step intervals, reducing the overall cost of the simulation. However, numerical heating of sub-critical density plasmas can occur with this implicit algorithm when $\omega_{p, e} \Delta t \lesssim 1$ (where the reverse holds true with cooling of supra-critical density plasmas with $\omega_{p, e} \Delta t \gtrsim 1$ ). Fortunately, for the durations of interest, this only amounts to a few $\pm 10 \mathrm{~s} \mathrm{eV}$ which is insignificant relative to direct heating by the laser to $\sim k e V$. Typically, simulations were performed with between 32 and 64 time steps per laser optical cycle, $T_{L}=\lambda_{L} / c$.

The particle push in LSP is energy-conserving, which is insensitive to numerical heating that is caused by under-resolving the Debye length where plasma electrons in a cell will artificially heat until the Debye length becomes the cell size [105]. For computational convenience, the Debye length not resolved in these simulations. Instead, convergence tests using increasingly finer mesh resolutions are performed under each simulation conditions to determine the minimal resolution necessary to study particular phenomena. For studying specular reflectivity with these relativistic lasers interacting with solid density plasmas, typically $\Delta x \lesssim \lambda_{L} / 16$ was necessary. In particular, spatial resolutions close to the skin depth of the laser fields at the interaction interface $\left(\delta_{s}\right.$, Equation 1.29) were found to be necessary for convergence of hot-electron properties. ${ }^{7}$

Even with all these simplifications of finite grid resolution and implementation of macroparticles, the simulations are still quite difficult to implement in a fully $3 D$ Cartesian geometry even on the world's largest supercomputers. Alternatively, to create a manageable number of macroparticles and grid size, $2 D$ Cartesian geometry is used with a virtual transverse dimension. The electric field is polarized in the interaction plane with

${ }^{7}$ For the quantities of interest, a resolution no larger than approximately twice the skin depth was needed. 
the magnetic field oriented in-and-out of the plane which, without any other events like scattering, can only move charges in the simulation plane ( $X Z$ in this case). Particles are instead 'rods' of charges where their electric fields fall off like $1 / r$ (where $r=\sqrt{x^{2}+z^{2}}$ ) instead of $1 / r^{2}$ which makes any phenomena sensitive to this Coulombic field falloff uncharacteristic of the experiment. LsP keeps track of particle momenta in the virtual dimension, known as $2 D 3 \mathrm{~V}$ geometry, but particles cannot be moved out of the plane and gradients of any quantity along this direction are always zero.

Some of the larger scale interactions I have modeled in this thesis consisted of spatial grids with $2752 \times 4800$ cells with upwards of $\sim 750$ million particles, modeled up to $\sim 2$ ps. Using 3840 processors for nearly 70 hours, some of the larger simulations required nearly $270 k C p u h$.

While LSP self-consistently solves Maxwell's equations and the Lorentz force with algorithms that have been extensively verified for stability and consistency under particular conditions, there is no guarantee that any resulting simulation will be characteristic, qualitatively or quantitatively, of the experimental conditions since not all of the physics is able to be modeled with infinite accuracy. Of particular relevance to this type of laser-plasma interaction, LSP has algorithms to approximate collisions, ionization and recombination, radiation generation and transport, relativistic electron energy losses (of particular interest to the transport diagnostics in these types of experiments), etc, but they each have limitations of their applicability and it's not a priori clear that the experimental observables are sensitive to any of them, individually or in conjunction. Experimental validation of each simulation condition is required to provide any viability to the study where sensitivity to each physical phenomena is typically addressed by simply running both with and without a particular model (for example, this approach is employed in Chapter 4 where the influence of dynamic ionization is studied). Due to the fluctuations in the experimental conditions and limited computational resources, only a limited number of simulations were performed under typical experimental conditions to infer the relevant physical phenomena in the interactions. 


\section{Chapter 2}

\section{LOW AND HIGH-CONTRAST RELATIVISTIC LASER-PLASMA INTERACTIONS EXPERIMENT}

In this Chapter, we ${ }^{8}$ discuss an experiment that studied the effects of pre-plasma on specular reflectivity measurements and hot-electron generation in relativistic laser-plasma interactions with solid density aluminum targets. In particular, we describe differences in the various properties of the incident and specularly reflected pulses (spectra, temporal profiles, spatial profiles, reflectivity) of both low and high-contrast pulse interactions. From these specular reflectivity measurements, we infer the influences of initial target conditions and, in subsequent chapters, constrain simulations used to indirectly study hot-electron generation in each scenario.

\subsection{Facility}

The experiment was performed on Titan at the Jupiter Laser Facility (JLF) at Lawrence Livermore National Laboratory (LLNL) in Livermore, CA. Titan is a two beam platform with a nanosecond scale 'long-pulse' and a picosecond $P W$ scale 'short-pulse', centered around $1.053 \mu \mathrm{m}$, which can be used independently or co-linearly with controllable relative delay. The second harmonic of both pulses is also available, generated using a $2 \mathrm{~mm}$ thick

\footnotetext{
${ }^{8}$ The experiment was designed and performed by a collaboration between various research groups at The Ohio State University, LLNL, UCSD, General Atomics, University of York and the University of Alberta. I helped develop and implement many of the laser, specular and transport diagnostics in the experiment and appropriate credit has been given to those whose analysis is included in this thesis.
} 
KDP crystal. Typical pulse parameters are shown in Table 2.1.

\begin{tabular}{lcccc}
\hline \hline & \multicolumn{2}{c}{ Long-pulse } & \multicolumn{2}{c}{ Short-pulse } \\
\hline$\lambda_{L}$ & $1053 \mathrm{~nm}$ & $527 \mathrm{~nm}$ & $1053 \mathrm{~nm}$ & $527 \mathrm{~nm}$ \\
Pulse & $0.35-20 \mathrm{~ns}$ & $0.35-20 \mathrm{~ns}$ & $0.7-200 \mathrm{ps}$ & $0.7-200 \mathrm{ps}$ \\
Max Energy & $1 \mathrm{~kJ}$ & $500 \mathrm{~J}$ & $300 \mathrm{~J}$ & $50 \mathrm{~J}$ \\
Best Focus & $20 \mu \mathrm{m}$ & $20 \mu \mathrm{m}$ & $8 \mu \mathrm{m}$ & $8 \mu \mathrm{m}$ \\
Intrinsic Pre-pulse & - & - & $15 \mathrm{~mJ}$ & $<10 \mu \mathrm{J}$ \\
\hline \hline
\end{tabular}

Table 2.1: Typical pulse characteristics of Titan, courtesy of the Jupiter Laser Facility website [106].

\subsection{Setup}

To consistently study the role of under-dense plasma in laser-generated hot electron sources, we designed the experiment to have a controllable amount of pre-pulse. The baseline case, i.e. minimal pre-plasma, used the high-contrast pulse at $527 \mathrm{~nm}$ produced via second harmonic generation (SHG) from the $1053 \mathrm{~nm}$ short pulse. Since SHG is a nonlinear process (i.e. the second harmonic signal is proportional to the square of the input intensity as it is a $\chi^{(2)}$ process), lower intensity portions of the pulse will not get converted as efficiently as the higher intensity portions resulting in a higher contrast pulse at the second harmonic $\left(2 \omega_{L}\right)$. The second harmonic of the long pulse was injected coaxially with the short pulse and was used as a controllable source of pre-pulse. Several diagnostics, as outlined in Figure 2.1, were used to study hot-electron generation and transport under these two conditions.

Although not critical for this study, the targets (Figure 2.2, assembled by General Atomics (GA) in San Diego, CA [107]) were designed to simultaneously accommodate a variety of transport diagnostics. The $C u$ tracer layer was used to track hot-electron current density via $K_{\alpha}$ radiation generated when hot-electrons that knock out k-shell electrons [70]. $2 D$ images of this $8.04 \mathrm{keV}$ emission were obtained using a spherically bent Bragg reflecting crystal but only within a narrow bandwidth ( $5 \mathrm{eV}$ Full-Width-at-Half-Maximum (FWHM)) 
Specular Diagnostics:

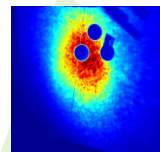

Reflectivity

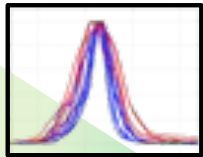

Spectrum

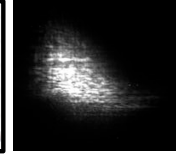

FROG

\section{Source/Transport Diagnostics*:}

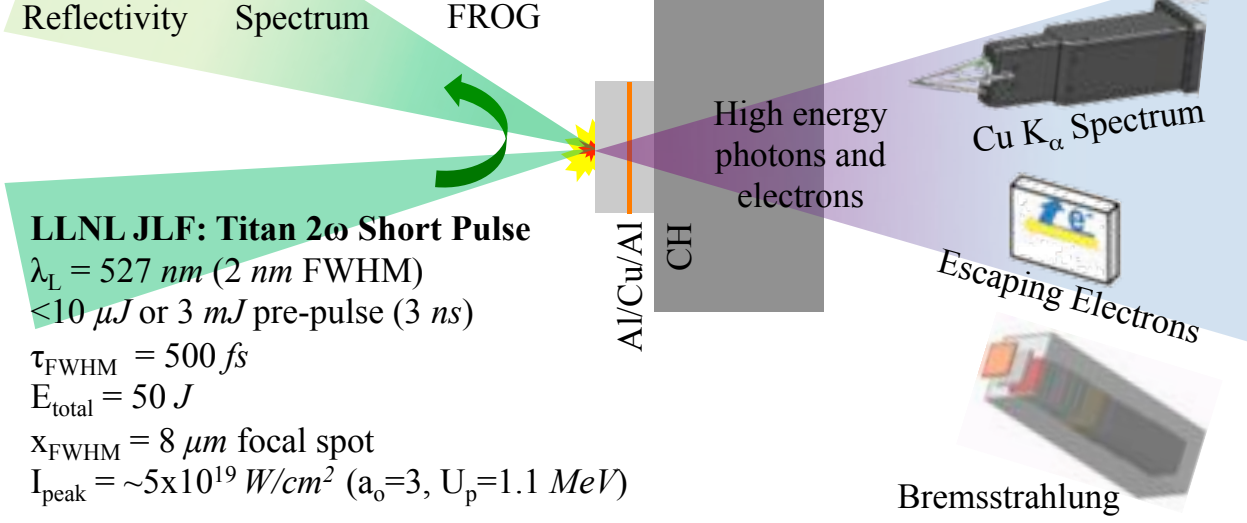

Figure 2.1: (Color) Experimental goals for high vs. low-contrast study on Titan: study effect of initial target conditions on hot-electron generation using a variety of optical, x-ray and electron diagnostics.

\section{Full Target Diagnostic Package}

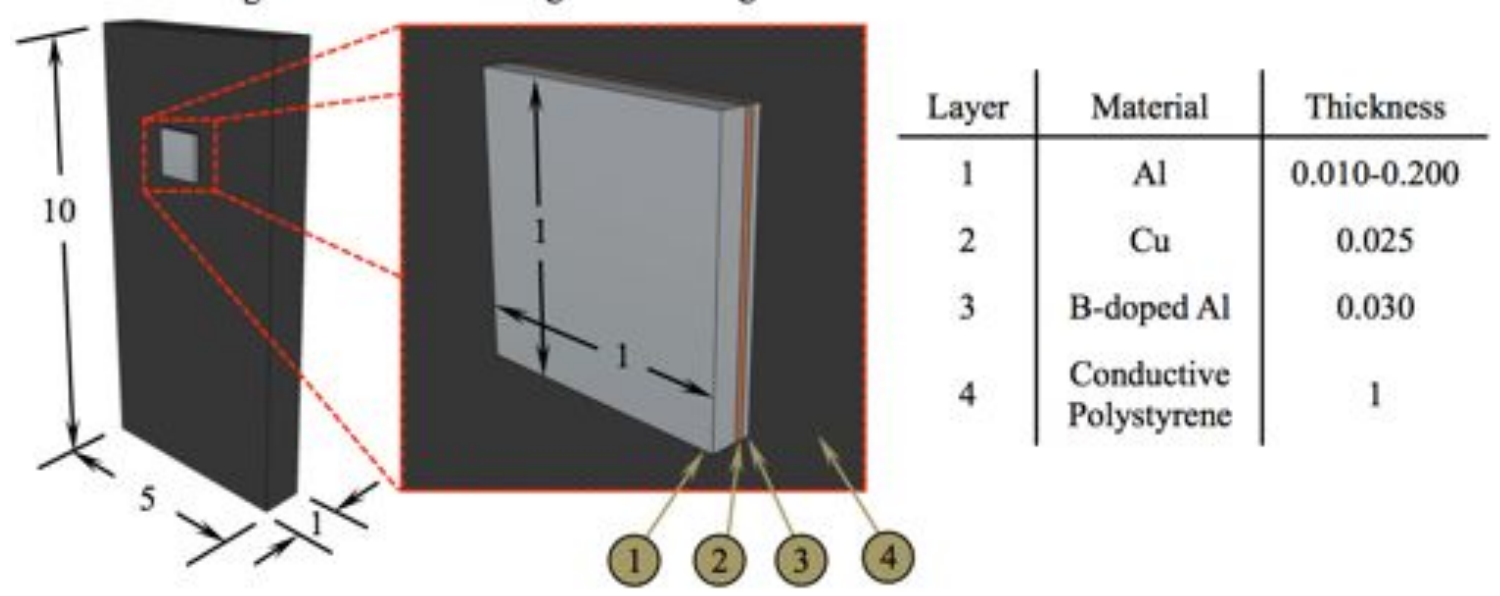

Figure 2.2: Target geometry for $2 \omega_{L}$ experiment (all dimensions are given in $\mathrm{mm}$ ). Front $A l$ layer (interaction surface) thickness varied from 10 to $200 \mu \mathrm{m}$. 
which can be sensitive to heating [71]. By varying the fluor depth (with the thickness of the first $\mathrm{Al}$ layer) and taking successive shots, an electron beam 'divergence' as it transports through the target was also inferred [25]. Integrated spectra of this K-shell emission was obtained using a calibrated Highly Ordered Pyrolytic Graphite (HOPG) spectrometer with range between $7.5 \mathrm{keV}$ and $13.3 \mathrm{keV}$ [65]. Escaping electron spectra were also obtained using a magnetic spectrometer. Although target charging effects can drastically modify the energy of hot-electrons as they leave the target and enter vacuum, modeling has been shown that there is a strong correlation between the slope temperature of the laser generated electron spectrum inside the target and that measured in vacuum [74]. Bremsstrahlung radiation created by these high energy electrons as they propagate through the target was also spectrally resolved (at various angles) and used to constrain the electron source and divergence $[27,64]$. As most of these diagnostics are sensitive to hot-electron refluxing, a conducting polystyrene layer $\left(1.04 \mathrm{~g} / \mathrm{cm}^{3}\right)$ was added to the back to minimize refluxing through the diagnostic fluor but with minimal x-ray signal attenuation. Fortunately, neither the $C u$ diagnostic fluor position or the GLL were found to have any observable effects on the specular pulse properties and will therefore not be discussed in any further detail.

Unfortunately, data collected in the high energy density sciences (HEDS) are often plagued by limited statistics as most of the platforms capable of reaching the desired relativistic conditions are capable of only a handful of shots a day. Of those few shots taken, there can be large fluctuations in laser pulse properties (energy, intensity distribution, duration, spectrum, etc) from one shot to the next. Worse still, electronic equipment used on such experiments can fail to acquire data due to electromagnetic interference (EMI) caused by high energy charged particles created during the shot. That being said, it is of upmost importance to fully characterize each and every shot as thoroughly as possible to be sure that any anomalous observations are not just a fluke; averaging over many shots is not advisable. Using rep-rated diagnostics, we characterized the incident and specularly reflected laser pulses in space, time and spectrum on each and every shot. Figures 2.3 and 2.4 show the experimental layout and setup for all the laser diagnostics (both incident and specular) for this experiment. 


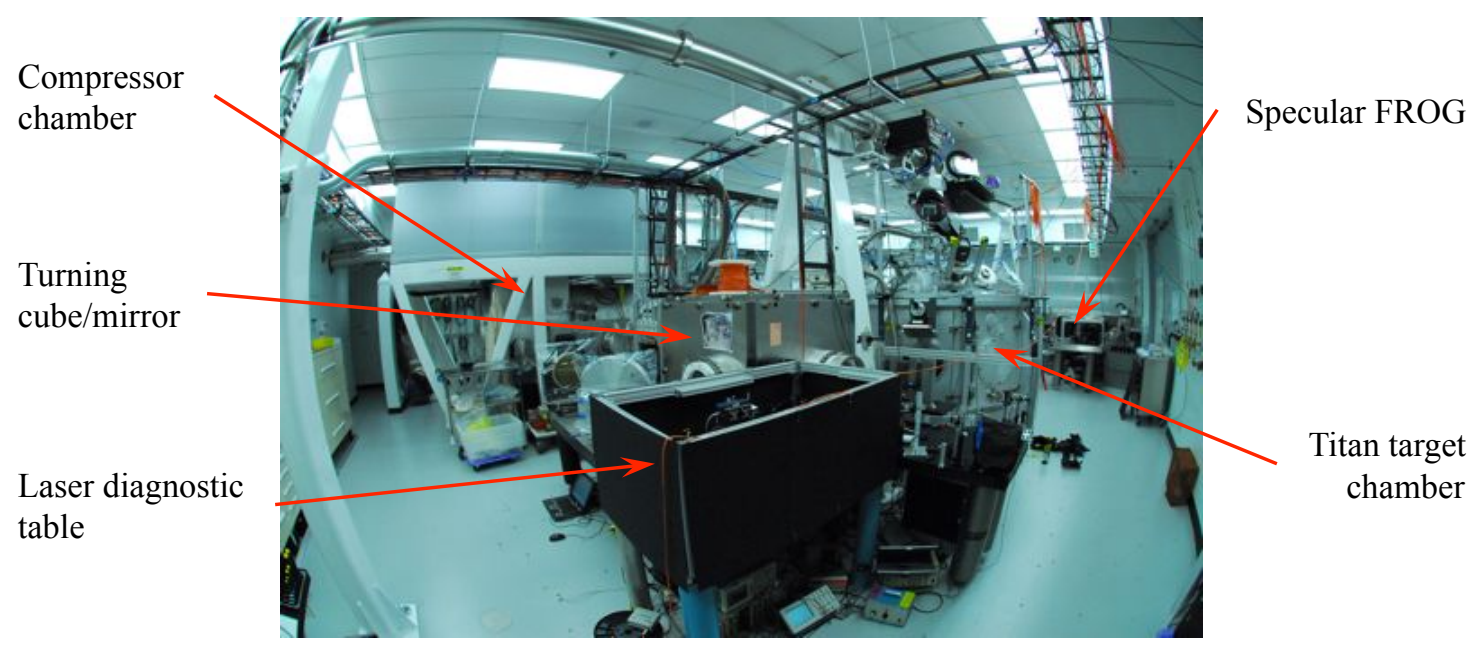

Figure 2.3: (Color) Titan target area at Jupiter Laser Facility (as viewed from a fisheye lens).

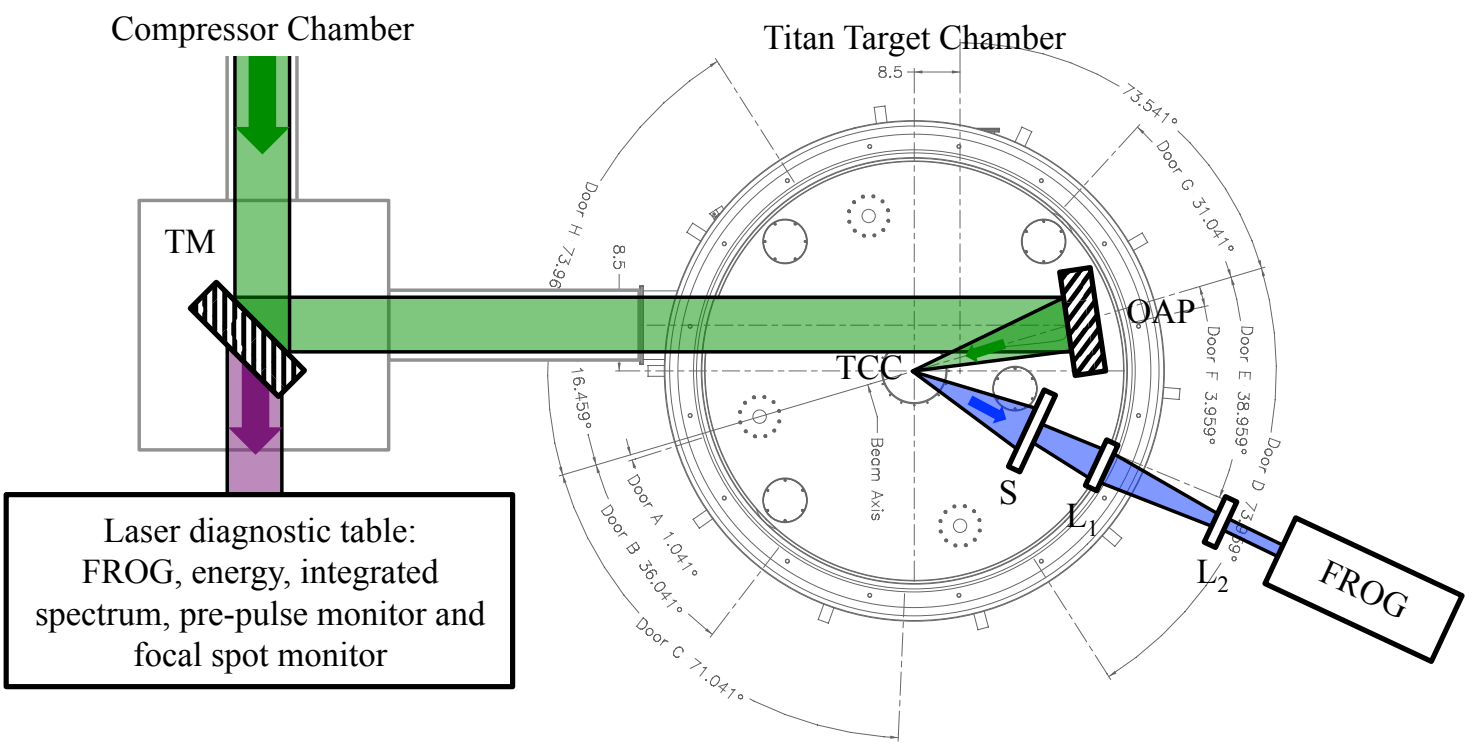

Figure 2.4: (Color) Experimental setup for high vs. low-contrast study on Titan. The green path is the incident pulse path (originating from the compressor chamber), purple the leakage through the last turning mirror used to characterized the pulse and blue the specular reflection. $T M=$ turning mirror, $O A P=$ off-axis parabola, $S=$ scattering plate and $L=$ lens. 
The incident pulse, originating from the compressor chamber, first reflects off the Turning Mirror $T M$ before being focused down onto the target (located at Target Chamber Center $T C C$ ) with an Off-Axis Parabola $O A P\left(15.3 \mathrm{deg}, f / 2.5^{9}, f=60 \mathrm{~cm}\right.$, path shown in green). The low intensity leakage through the $T M$ (purple) was sent to the laser diagnostic table where the energy, temporal and spatial profiles, spectrum and pre-pulse of the incident laser pulse were measured. A detailed schematic of the exact layout of the laser diagnostic table is provided in Appendix A. The pre-pulse monitor was capable of measuring pre-pulse energies down to $\sim 10 \mu J$ with the help of a nonlinearly absorbing medium and is discussed in more detail in Appendix B. The temporal profile of the laser pulse, both intensity and phase, was obtained using a technique known of Frequency Resolved Optical Gating (FROG) [108] which is discussed in some detail in Appendix C. In short, a FROG measurement is an autocorrelation of the pulse with itself, resolved in frequency, resulting in a $2 D$ spectrogram in wavelength $\lambda$ and relative delay $\tau$. Using an iterative phase retrieval algorithm, the temporal profile of the complex electric field that created the spectrogram can, in theory, be uniquely retrieved [109]. The on-shot energy was obtained using a calibrated calorimeter and the on-shot focal spot was also monitored, but unfortunately it wasn't available for the shots of interest. Instead, the on-shot intensity distribution was estimated using the spatial distribution of a using a low energy shot obtained at best focus at $T C C$.

Similar characteristics of the specularly reflected pulse were also measured, albeit in a slightly different manner with the biggest difference being that these measurements were obtained in the far-field where diffraction effects have fully manifested themselves. Collecting the light scattered from a diffuse reflector screen, we captured the time-integrated far-field spatial distribution, obtained an energy reflectivity measurement as well as the integrated spectrum of the specularly reflected pulse. Using a small hole in the screen, we sent a small fraction of the central part of the beam to a FROG for the temporal intensity and phase profile.

\footnotetext{
${ }^{9}$ The $f / \#$ is the ratio of the optical systems focal length to the beam diameter and is a measure of beam convergence
} 


\subsection{Typical pulse characteristics}

Variability in the data (and unreliability of electronics on shot because of EMI) resulted in only 2 data shots being discussed in this thesis. As the focus of this experiment is about controlling the pre-plasma, I begin the laser pulse characterization with a pre-pulse measurement. Pre-pulse measurements [110] of the two shots of interest are shown in Figure 2.5, using the pre-pulse monitor design discussed in Appendix B. In short, nonlinear absorbing media (one cell with $\mathrm{CS}_{2}$ and another with $\mathrm{PbCHCl}_{3}$ ) were used as optical limiters to protect the photodiode from the high intensity portion of the pulse, increasing the dynamic range of the instrument and sensitivity to the pre-pulse signal.

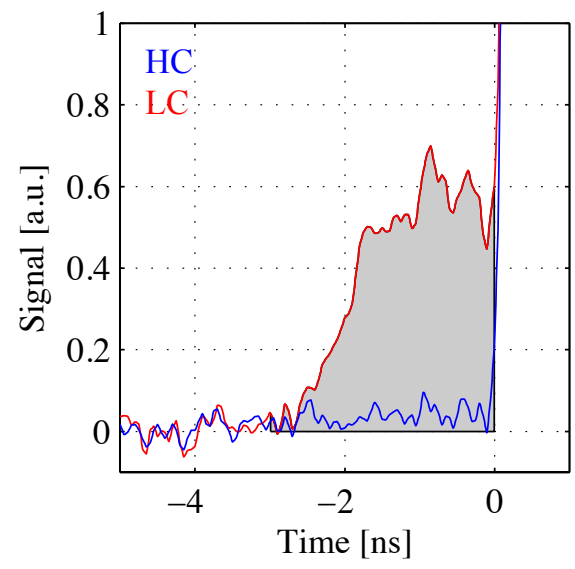

Figure 2.5: (Color) Typical pre-pulse measurement of both the high-contrast (HC) shot in blue and the low-contrast (LC), injected pre-pulse shown in red. The high-contrast shot was found to be below the detection threshold due to an artifact in the scope trace. The low-contrast shot had $3.16 \pm 0.12 \mathrm{~mJ}$ delivered in the $3 \mathrm{~ns}$ before the main pulse arrived (at time equal to 0 ps when the signal saturates).

By integrating the area under the photodiode signal, the amount of energy in the prepulse was measured for both the high-contrast (blue) and low-contrast (red) shots. ${ }^{10}$ From

\footnotetext{
${ }^{10}$ The noisy high-contrast signal was found to be merely an artifact of the scope where the pre-pulse was
} found to be below the detection threshold of the instrument. 
this measurement, the intrinsic pre-pulse energy was estimated to be less than $10 \mu \mathrm{J}$. In the 3 ns before the short pulse arrived (at time equal to 0 ps when the signal saturates), approximately $10 \mathrm{~J}$ of the $1053 \mathrm{~nm}$ long-pulse resulted in $3.16 \pm 0.12 \mathrm{~mJ}$ of $527 \mathrm{~nm}$ prepulse after the second harmonic conversion. The error bars were obtained by the quadrature addition of the background noise (integration over $3 \mathrm{~ns}$ of the background signal) and integration window offset (shifting the window by \pm 1 pixel).

The incident laser pulse was characterized using a PG FROG ${ }^{11}$ (Appendix C) and the resulting spectrogram measurement of the high-contrast incident pulse is shown in Figure 2.6(a) (as $\sqrt{I(\lambda, \tau)}$ to emphasize low energy portions of the trace). The temporal intensity and phase information was retrieved using the iterative phase retrieval algorithm discussed in Appendix C.2. Using a $512 \times 512$ grid, FROG errors (defined to be the RMS difference between the original trace and retrieved one, Equation C.4) were found to be a few percent. The lowest retrieved FROG error reconstruction of the incident laser pulse is shown in Figure 2.6(b) with Figures 2.6(c) and 2.6(d) the time and wavelength domains of the reconstructed pulse respectively. For both domains, the instantaneous intensity (black) and phase (red) are simultaneously displayed.

Since these experimentally obtained traces aren't ideal FROG traces (i.e. they have some uncertainty due to noise, calibration, geometrical effects etc), running the algorithm on multiple occasions with different initial seeds results in similar, but not identical, reconstructions all with equally low FROG errors. Given enough reconstructions, a careful calculation of the mean and standard deviation of all the traces can indicate the consistency of the reconstruction (procedure discussed in Appendix C.4) and provide a retrieval error estimate. Shown in Figure 2.7 are the error weighted, mean and standard deviation of 100 reconstructions for this laser FROG trace.

Figure 2.7(a) is the temporal intensity profile (black) with the gray shaded area indicating a $\pm \sigma_{S T D}$ deviation about the mean. Unfortunately, the exact structure that appears in the FROG trace is not uniquely determined as apparent from the shaded gray area.

\footnotetext{
${ }^{11}$ These PG FROGs had approximately a $10 \mathrm{ps}$ total delay window with $\approx 125 \mathrm{fs}$ resolution and approximately a $100 \mathrm{~nm}$ spectral window.
} 

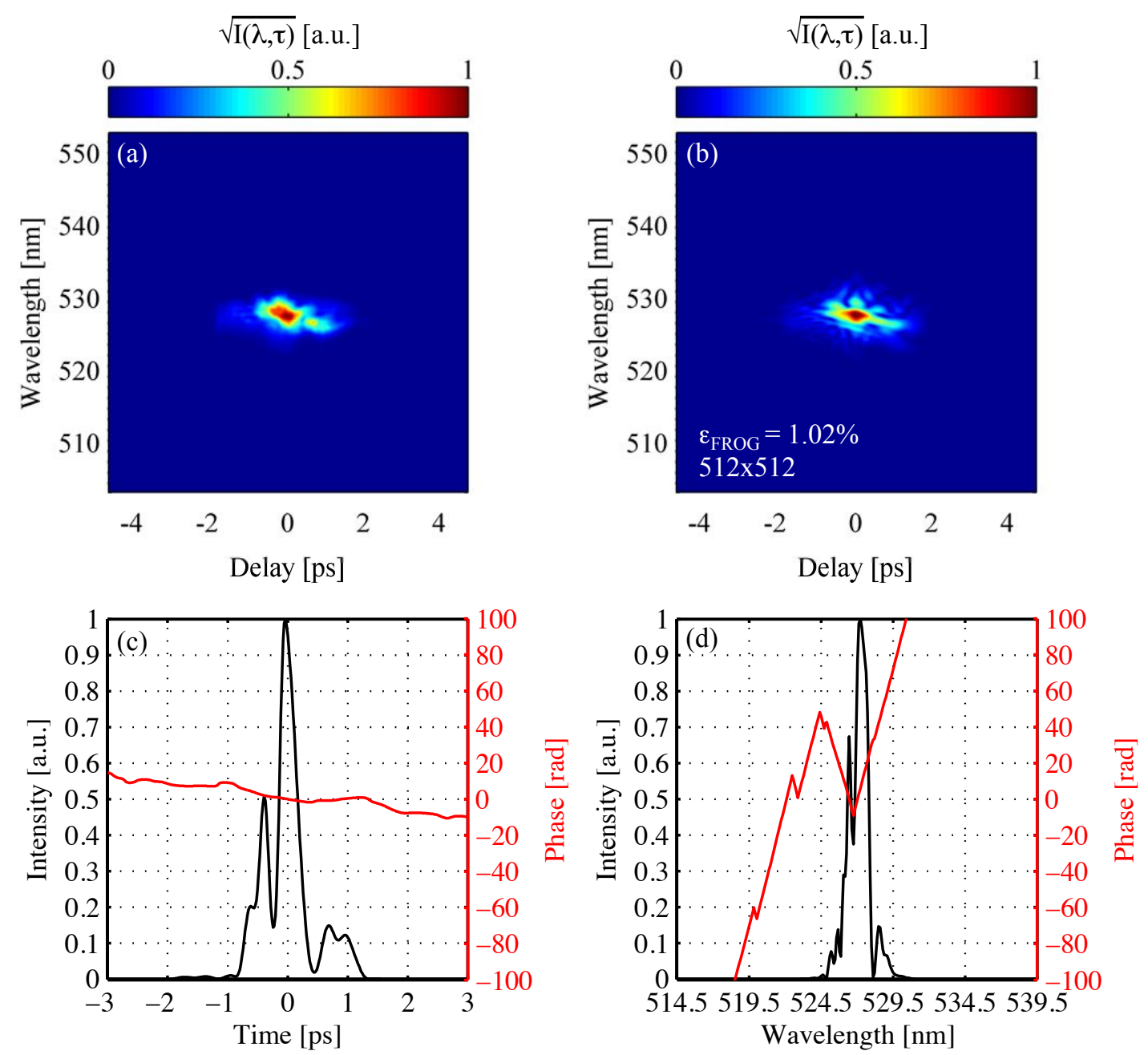

Figure 2.6: (Color) Typical laser FROG trace and reconstruction. Subplot (a) is the experimental data, shown as the square root of the signal to emphasize low energy portions of the trace. Subplot (b) is the best retrieved trace out of 100 reconstructions with subplot (c) showing the temporal intensity and phase profile and (d) the spectral profile.

This pulse was found to have approximately a $460 \mathrm{fs}$ temporal intensity FWHM with a lower intensity post-pulse centered nearly 1 ps after the main pulse. The main pulse width varied between approximately 400 and $700 \mathrm{fs}$ FWHM throughout the experiment. More physically intuitive than a mean phase with error bars, the red curve (shaded pink) shows the shift in the instantaneous wavelength $\lambda_{\text {inst }}=2 \pi c / \omega_{\text {inst }}$ (Equation 2.1) with respect to the carrier wavelength $\left(\lambda_{L}=527 \mathrm{~nm}\right)$ using the methods discussed in Appendix C.4. 

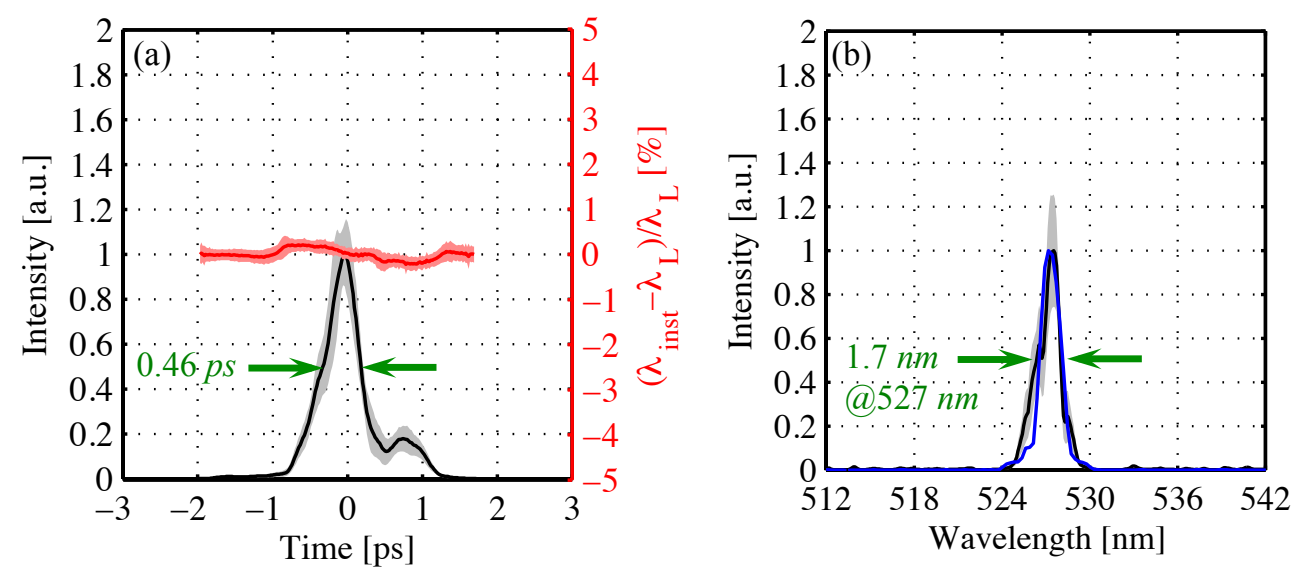

Figure 2.7: (Color) Averaged laser FROG reconstruction. Subplot (a) contains the instantaneous intensity (black) and wavelength (red) and subplot (b) contains the average spectral intensity (black) and an independent spectrum measurement (blue). These curves are the error weighted average of 100 separate retrievals and the error bars (shaded gray area for intensity, red error bars for instantaneous wavelength shift) are $\pm \sigma_{S T D}$ about the mean.

Instantaneous wavelength shifts corresponding to intensities below approximately $1 \%$ of the peak intensity are truncated as the phase retrieval is found to be increasingly unreliable in this region. Instantaneously wavelength shifts about the $527 \mathrm{~nm}$ carrier were found to be less than $\pm 0.25 \%$.

$$
\Delta_{\lambda}=\left(\lambda_{\text {inst }}-\lambda_{L}\right) / \lambda_{L}
$$

Similarly, spectral intensities are shown in $2.7(\mathrm{~b})$ where the intensity is again shown in black and standard deviation error bars in gray. An independent measurement of the spectra (using a fiber-coupled Ocean Optics ${ }^{\circledR}$ HR4000 spectrometer [111]) is shown in blue and is found to be within the error bars of the retrieval. The spectrum has approximately a $1.7 \mathrm{~nm}$ spectral intensity FWHM. For comparison, a transform limited (i.e. $\left.\omega_{\text {inst }}(t)=\omega_{L}\right)$ Gaussian pulse with a $460 \mathrm{fs}$ FWHM centered around $527 \mathrm{~nm}$ would have a spectral FWHM of about $0.9 \mathrm{~nm}$ (alternatively, a transform limited Gaussian pulse with $1.7 \mathrm{~nm}$ bandwidth would be closer to $240 \mathrm{fs}$ ). This pulse is clearly not transform limited indicating that the pulse is chirped, i.e. the instantaneous wavelength varies throughout the pulse. 
Fortunately, this amount of chirp is a negligible effect for our application as will be discussed shortly in the specular pulse properties in Chapter 2.5.

The next laser property of interest is the energy of the main pulse. This information was obtained using a calibrated Molectron calorimeter [112] on the laser diagnostic table (labeled as Calorimeter in Figure A.1). Fortunately for the shots of interest here, the energy was stable around $35 \mathrm{~J}$, but varied between 31 and $53 \mathrm{~J}$ throughout the experiment.

Many effects in relativistic laser-plasma interactions, such as self-focusing for example (Chapter 1.5.4), also depend on the spatial intensity distribution of the incident laser pulse. The pulse was focused down using an off-axis parabola $O A P(f / 2.5,13.5 \mathrm{deg}, f=60 \mathrm{~cm})$ onto the target at Target Chamber Center $T C C$ with the electric field polarized horizontally (p-polarized interaction with the target). Since a focal spot obtained at best focus cannot be obtained on a full energy shot (since there happens to be a target in the way and it would also destroy the camra), an equivalent on-shot focal spot distribution was obtained using the full aperture leakage through the last turning mirror, referred to here as the focal spot monitor (beam path outlined in Figure A.1). Instead of an equivalent imaging system (i.e. an identical $O A P$ to the one used in target chamber), an $f / 25$ lens $(f=6.1 \mathrm{~m}$ at $527 \mathrm{~nm})$ was used to help mitigate alignment issues associated with small f-number $(f / \#)$ focusing optics (mainly the depth-of-focus being directly proportional to $\left.(f / \#)^{2}\right)$. The sequential reflection off of two uncoated wedges was used to reduce the energy of the pulse on the laser diagnostic table so as to not destroy the CCD detector (Apogee ALTA U47+UV [113], $1024 \times 1024$ array, $13 \times 13 \mu m^{2}$ pixels). However, the focal spot size is also directly proportional to the $f$ /\# so an additional correction (given simply by the ratio of the $f$ /\#'s of the $O A P$ and lens) was necessary for the equivalent on-shot focal spot distribution on target.

Unfortunately, this focal spot monitor was not available for the shots of interest to this thesis. Instead, a low energy focal spot obtained at TCC using a microscope was used to estimate the on-shot focal spot. A typical low energy focal spot obtained at TCC is shown in Figure 2.8(a) [114]. Although it is irregular, an equivalent circularly symmetric focal spot was created (using the high-contrast shot with $33 \mathrm{~J}, 460 \mathrm{fs}$ ) by fitted using a superposition 
of 3 Gaussians to estimate the full power, on-shot intensity distribution. This intensity distribution is shown in Figure 2.8(b) and its Gaussian components listed in Figure 2.8(c). Ultimately, we estimate for these shots our focal spot on target has a peak cycle averaged intensity of approximately $4.6 \times 10^{19} \mathrm{~W} / \mathrm{cm}^{2}$ delivered into an $8 \mu \mathrm{m}$ FWHM focal spot $[114]$.
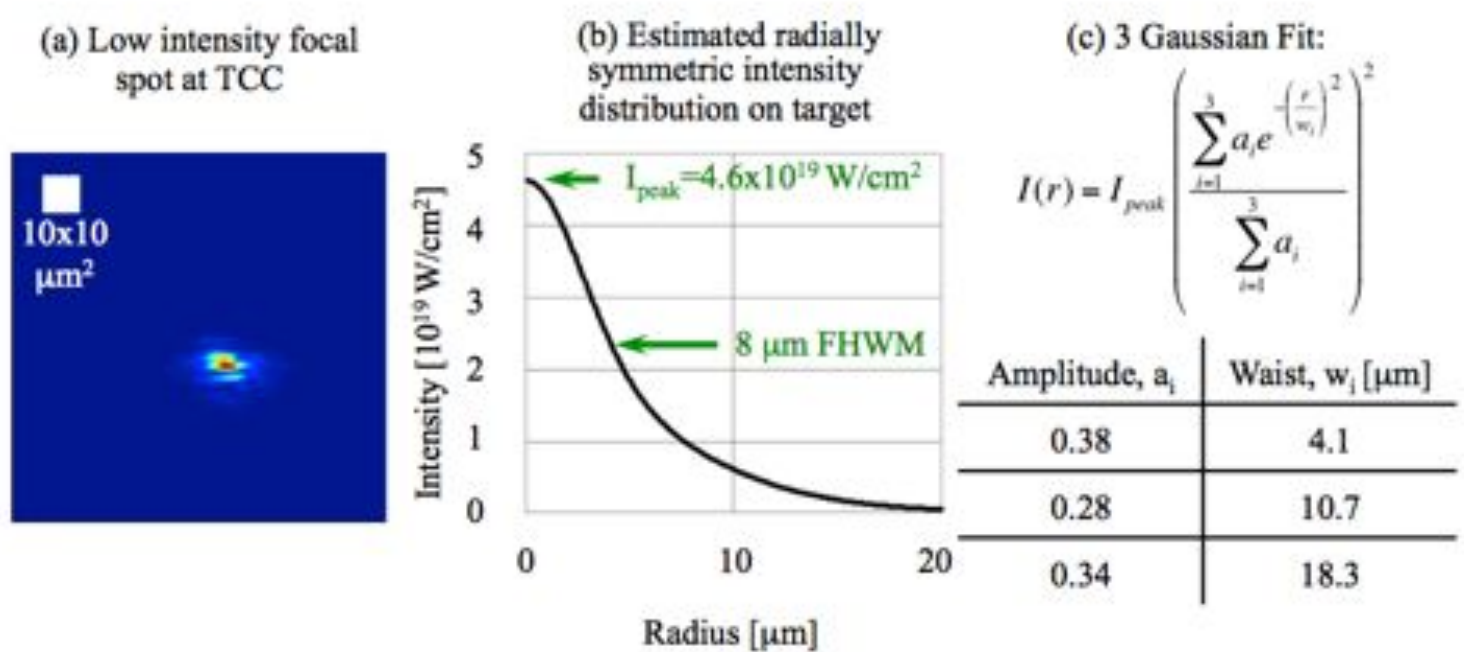

Figure 2.8: (Color) Focal spot intensity distribution [115]. Subplot (a) is a low intensity focal spot obtained at TCC. Subplot (b) is the estimated, radially symmetric, on-shot intensity distribution ( $33 \mathrm{~J}, 460 \mathrm{fs}$ ) using the energy distribution in subplot (a). Peak intensities observed were $\sim 4.6 \times 10^{19} \mathrm{~W} / \mathrm{cm}^{2}$ with an $8 \mu \mathrm{m}$ FWHM focal spot. Subplot (c) shows the components of a 3-Gaussian fit to the radially averaged intensity distribution in subplot (b). Courtesy of S. Kerr [114].

This injected pre-pulse (co-axially aligned with the short pulse) was focused down using the same parabola however only down to approximately a $14.1 \mu \mathrm{m}$ focal spot with peak cycle averaged intensities of around $6.38 \times 10^{11} \mathrm{~W} / \mathrm{cm}^{2}$ [115]. The pre-pulse to main pulse energy contrast ratio was therefore $<2 \times 10^{-7}\left(6 \times 10^{-5}\right)$ and intensity contrast ratio was $<4.6 \times 10^{-11}\left(1.4 \times 10^{-8}\right)$ for the high (low) contrast pulse. 


\subsection{Initial target conditions}

While neither the $C u$ diagnostic fluor position or the $C H$ GLL were found to have any observable effects on the specular pulse properties, the variety of surface roughness' shot during the experiment were found to have a dramatic effect on the high-contrast reflectivity measurements. While the scales of these surface perturbations were unlikely to effect lowcontrast pulse shots, i.e. the pre-pulse would likely 'wash' out any of these features leaving an extended pre-plasma environment, high-contrast pulses have been found to be quite sensitive to even wavelength scale surface perturbations. In this section, we discuss the characterization of the initial target conditions just before the main pulse arrives for both the low and high-contrast shot conditions.

\subsubsection{Low-contrast}

For the low-contrast, injected pre-pulse shots, radiation hydrodynamic calculations were performed to estimate the pre-plasma environment conditions. The calculation was performed using MULTI2D [116] and was carried out by Rafael Ramis at Universidad Politécnica de Madrid (UPM). The pre-pulse was modeled as $15 \mu \mathrm{m}$ FWHM gaussian spatial profile with a $3 \mathrm{~ns}$ flat top temporal profile, containing $3 \mathrm{~mJ}$ of energy incident normally on an initially cold solid density $A l$ target with cylindrically symmetric $R Z$ geometry. Figure $2.9(\mathrm{a})$ is the total electron density on a $\log _{10}$ scale (normalized to the critical electron density $n_{e} / n_{c}$ ), (b) the cell averaged ion charge state and (c) the electron temperature in $\mathrm{eV}$ at $3 \mathrm{~ns}$. For these figures, the original cylindrically symmetric $R Z$ result was mirrored about the $r=0 \mu m$ plane. Absorption at these intensities $\left(\lesssim 6.38 \times 10^{11} \mathrm{~W} / \mathrm{cm}^{2}\right)$ is largely dominated by collisional processes [85, 90] and nearly all of the absorption in the simulation was attributed to inverse Bremsstrahlung [85, 115].

Originally, the solid density aluminum interface $\left(n_{e} / n_{c} \sim 45\right)$ was perfectly flat (initially the $x=0 \mu m$ plane). Now, 3 ns later and just before the main pulse would have arrived, the pre-pulse has sufficiently heated the front of the target enough to 'dimple' the original solid density interface by nearly $5 \mu \mathrm{m}$ along with creating under-dense plasma that extends 

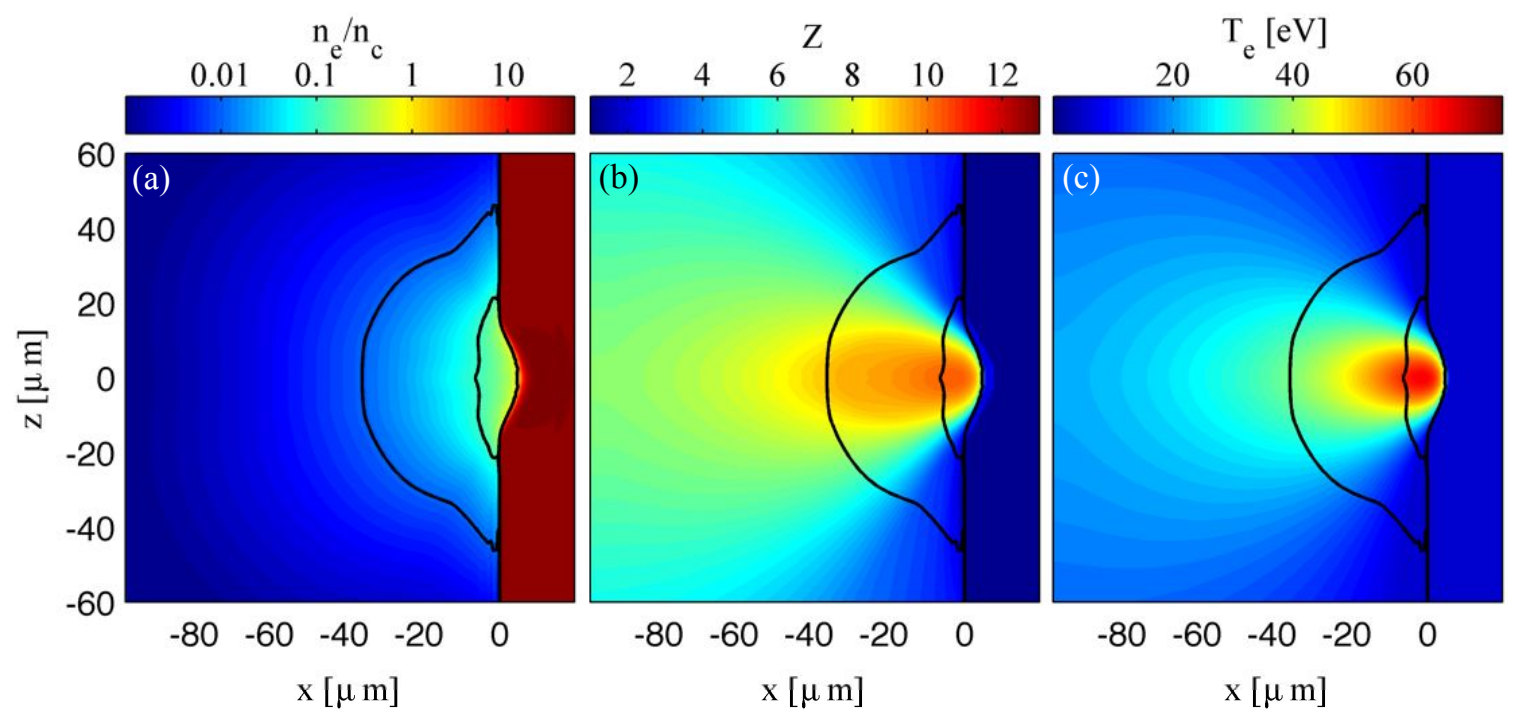

Figure 2.9: Estimated pre-plasma environment using a 2D radiation hydrodynamics Lagrangian code MULTI2D at 3 ns. Subplot (a) is the electron density (normalized to the critical density) on a $\log _{10}$ color scale, subplot (b) is the ion charge state and subplot (c) is the electron temperature in $e V$. The 3 black curves in all subplots are the $n_{c}, n_{c} / 10$ and $n_{c} / 100$ contours.

\begin{tabular}{ccccc}
\hline \hline$n_{e} / n_{c}$ & $x[\mu m]$ & $L[\mu m]$ & $Z$ & $T_{e}[\mathrm{eV}]$ \\
\hline 1 & +4.7 & 1.3 & 5.5 & 30 \\
0.1 & -6.5 & 10.6 & 10.2 & 62 \\
0.001 & -36.8 & 18 & 9.1 & 36 \\
\hline \hline
\end{tabular}

Table 2.2: Summary of pre-plasma environment from the MULTI2D calculation along symmetry axis.

out nearly $100 \mu \mathrm{m}$ with spatially varying charge state and temperature distributions. The pre-plasma electron density profile can be described by various exponential density profiles depending on where you look. Some of these characteristics are summarized in Table 2.2 at various electron densities (relative to the critical density) using a line-out of these various quantities along the symmetry axis $(z=0 \mu m)$. The location $x$ of these densities are given along with the local exponential scale length $L$ where $n_{e}(x) \propto \exp (-x / L)$. Also shown are the local charge state $Z$ and electron temperature $T_{e}$. Although the under-dense plasma 
10 's of microns from solid density is nearly $10 \times$ ionized, these simulations would suggest that the initial pre-plasma near the critical density would not be even be close to fully ionized. Therefore, dynamic field ionization could significantly alter the interaction region, propagating laser pulse (both discussed in Chapter 1.5.4) as well as specular reflectivity measurements.

\subsubsection{High-contrast}

In high-contrast, short pulse laser-plasma interactions, target surface morphology can matter. For targets with initial surface perturbations (i.e. roughness), significant enhancements in absorption have been reported which have typically been attributed to enhanced Brunel [72, 98], resonant excitation of surface plasma waves [117-120] or local field enhancement via Mie resonance $[121,122]$ depending on the specifics of each study. Therefore, great care has been taken to characterize the experimental targets surface morphology on both laser spot size $(\sim 8 \mu \mathrm{m})$ and a laser wavelength $(<527 \mathrm{~nm})$ scales.

Unfortunately, only the few targets not shot during the experiment survived to be characterized. Atomic Force Microscope (AFM) [123] measurements of similar targets were made twice for each target: (1) 'large scale' features at $390 \mathrm{~nm}$ resolution over a $100 \times$ $100 \mu \mathrm{m}^{2}$ box to see fluctuations over the focal spot size and (2) 'small scale' features at $19.5 \mathrm{~nm}$ resolution over a $10 \times 10 \mu \mathrm{m}^{2}$ to see deviations on a sub laser wavelength $\lambda_{L}$ scale. $^{12}$ Two typical target scans (at the lower resolution) are shown in Figures 2.10(a) and (b) and clearly several different features were observed. Subplot (a) is typical of the Goodfellow foils [124] used for the shallowest fluors and had an nearly isotropic spectral intensity distribution with an RMS value of around $400 \mathrm{~nm}$. To obtain deeper $\mathrm{Cu}$ tracer fluor layers, the Goodfellow foils were electroplated by GA (subplot (b)) where a preferential groove orientation was observed but generally with much smoother overall with RMS values ranging between 100 (vertically) and $200 \mathrm{~nm}$ (horizontally). The corresponding spectra of these targets (as a function of wave-number $k=2 \pi / \lambda$ ) are shown in (c) where the shaded gray area indicates the range of the data. To obtain variations on a sub-wavelength scale, a

\footnotetext{
${ }^{12}$ The AFM measurements were performed by students of R. Fedosejevs at the University of Alberta.
} 
low-wavelength pass edge filter (at $\sim 4 \lambda_{L}$ ) was applied to the higher resolution data. Subplot (d) shows the single unique small scale feature that appeared across all these targets with an average RMS of $25 \mathrm{~nm}$ (again with the mean in black and the shaded gray area the data range). The targets, in reality, had a superposition of these two spectrum.

\subsection{Specular pulse properties}

Once again, variability in the data and unreliable electronics resulted in only two data shots being discussed in this thesis: one low-contrast shot with the injected pre-pulse $(\sim$ $3 \mathrm{~mJ}, 3 \mathrm{~ns})$ and the other high-contrast shot the intrinsic pre-pulse $(<10 \mu J)$. Just like the incident laser pulse, the total energy, spatial and temporal profiles and spectrum of the specularly reflected pulses were also measured, albeit in slightly different ways. The largest difference between the incident and specular pulse characterization is that the specular pulse properties were all measured in the far-field, $10 \mathrm{~s}$ of $\mathrm{cm}$ away from the best focal position where diffraction effects have been fully manifested.

\subsubsection{Integrated reflectivity and spectrum}

By knowing the laser energy that is incident upon a target and the energy that is specularly and diffusely reflected, we can place an upper bound on the amount of energy that could be transferred into high energy electrons created during the interaction, a critical parameter in any laser-plasma interaction. The integrated spectra, total energy and $2 D$ spatial profile of the specularly reflected pulse were all obtained with the help of a calibrated scattering plate made of optical grade Spectralon ${ }^{\circledR}$ produced by Labsphere ${ }^{\circledR}$, Inc. [125].

Spectralon ${ }^{\circledR}$ is a fluoropolymer, similar to Teflon, which is a nearly perfect diffuse reflector for the ultraviolet to near-infrared regions of the spectrum, scattering $>99 \%$ of the incident light into $2 \pi$ steradians [126]. It also exhibits extremely Lambertian properties for visible and near-infrared spectrums. By definition, this means that the number of photons $d N_{\omega}$ scattered per unit time $d t$ into solid angle $d \Omega$ by an emittance area $d A$ ) varies linearly with the cosine of the viewing angle $\theta$ (taken with respect to the surface normal). This 

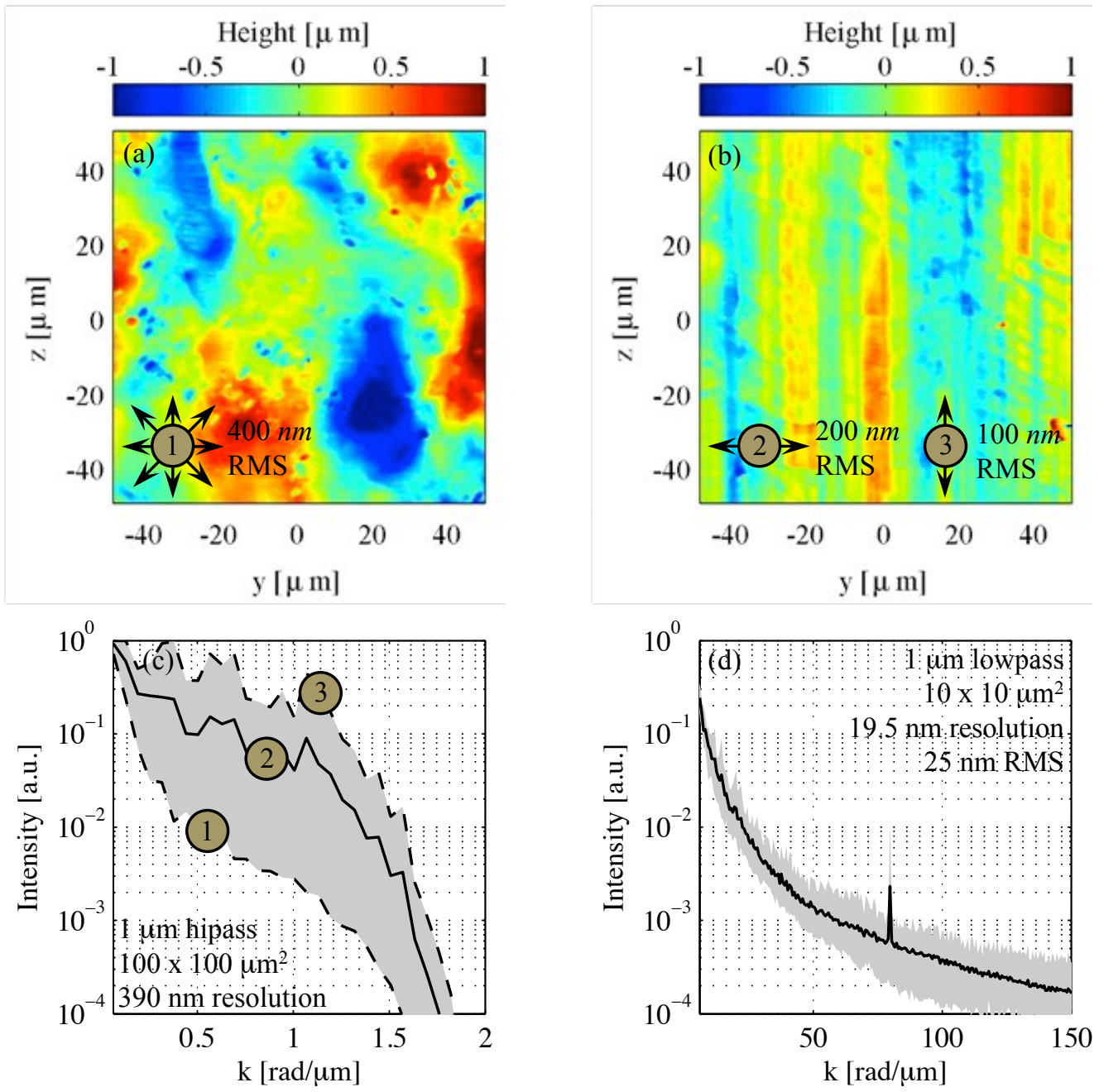

Figure 2.10: Typical target surface roughness' for $2 \omega_{L}$ experiment. Subplots (a) and (b) are AFM measurements of typical targets shot for this experiment where (a) is typical of the $10 \mu \mathrm{m}$ thick aluminum front layer targets and (b) the thicker 50-100 $\mu \mathrm{m}$ deep fluor layers. Surface (a) is more-or-less isotropic with a $400 \mathrm{~nm}$ RMS. Surface (b) has a preferential groove pattern along one dimension. The shaded gray area indicated the range of the data. Subplot (d) shows the spectrum of a higher resolution scan of these same targets, but with a $2 \mu \mathrm{m}$ hipass (frequency) edge filter. At this scale all targets showed similar RMS and distribution.

is illustrated in Figure 2.11(a), where the number of photons per unit time emitted into each wedge is $I \cos \theta d \Omega d A$ with $I$ the incident irradiance (i.e. brightness). For different observers looking at the same emittance area, the apparent irradiance of the scattered light $I_{o}$ is independent of viewing angle since the solid angle of the observer $d \Omega_{o}$ also varies as 
$\cos \theta$, illustrated in Figure 2.11(b) [127].
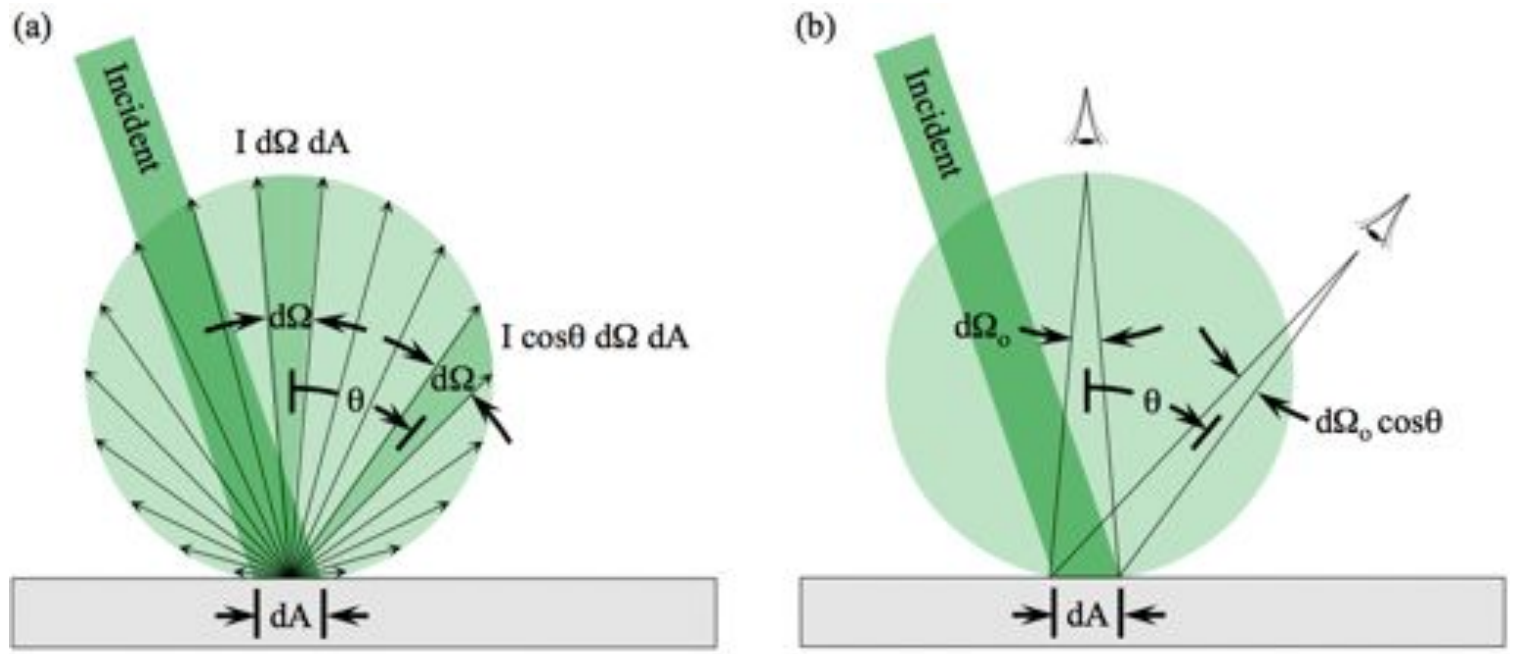

Figure 2.11: Lambertian scattering surface showing (a) angular dependence on scattered photon emission and (b) isotropic radiance [127].

The $25.4 \times 25.4 \mathrm{~cm}^{2}$ screen was $35.2 \mathrm{~cm}$ away from $T C C$ (normal to the specular laser propagation axis) resulting in an $f / 0.74$ collection window (labeled as $L$ in Figure 2.4). By imaging the light scattered off of this surface at some angle relative to the Spectralon ${ }^{\circledR}$ normal, we obtain a time integrated $2 D$ map of the intensity distribution along with a means for measuring the energy of the incident radiation. The imaging system used a variety of Schott glass neutral density (ND) optical glass filters as well as a $50 \mathrm{~nm}$ FWHM interference filter centered around $\sim 532 \mathrm{~nm}$ to keep the imaging camera within the linear response range. For the CCD camera used in the camera, a 16-bit Andor DV434 [128], the linear regime was below approximately $40 k$ counts for green light. The spectrally resolved transmission of the individual filters are shown in Figure 2.12(a) along with the systems total (b) optical density $(\approx 5.2)$ and $(\mathrm{c})$ transmission curves.

Since the power scattered into any particular direction with respect to the surface normal will vary as $\cos \theta$ (Figure 2.11(b)), an in situ calibration was necessary to convert the imaged 
scattered light signal into an incident energy. Using a shuttered, continuous-wave (CW) green diode laser $(532 \mathrm{~nm})$ with known energy per shutter interval and incident on the screen at the same angle as the specular pulse, a calibration to convert from pixel count to energy was obtained (Figure 2.12(d)), resulting in $9.54 \pm 0.79 \mathrm{~nJ} /$ count. The calibration curves and analysis in Figure 2.12 were courtesy of A. Link [129].
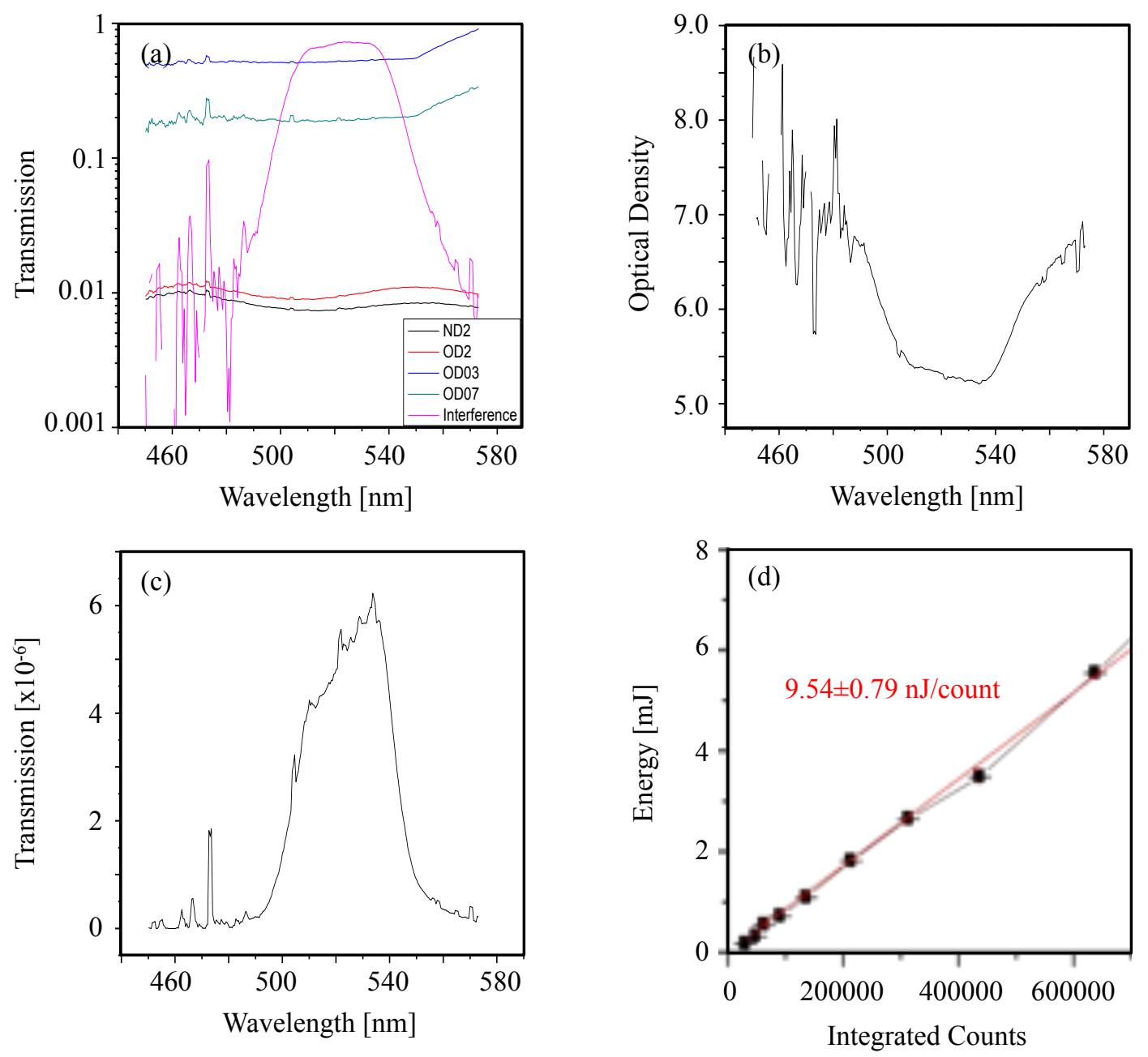

Figure 2.12: (Color) Spectralon filter and energy calibration courtesy of A. Link [129]. Spectrally resolved (a) individual filter transmission curves, (b) effective optical density of the complete filter set and (c) the total transmission curve. (d) In-situ calibration using a shuttered CW green diode laser $(532 \mathrm{~nm})$. 
In order to take the spectral response of the imaging system into account for the energy calibration, the integrated spectra of the specular pulse was simultaneously measured by collecting the light scattered off the screen with a calibrated fiber-coupled Ocean Optics ${ }^{\circledR}$ USB2000 mini spectrometer [130]). The high and low-contrast spectra are shown in Figure 2.13 where the incident laser pulse spectra is shown in black, the high-contrast in blue and the low-contrast in red. Immediately, we observe $\gtrsim 2.5 \times$ broader integrated spectrum $(\approx 4.2 \mathrm{~nm}$ FWHM) and an overall $1 \mathrm{~nm}$ blue shift in the carrier $(526 \mathrm{~nm})$ compared to the incident pulse (1.7 $\mathrm{nm}$ FWHM, $527 \mathrm{~nm}$ carrier) for both shots. However, for the lowcontrast shot, we notice an increased contribution from 'redder' portions of the spectrum $(\lambda>527 \mathrm{~nm})$ as compared to the high-contrast shot.

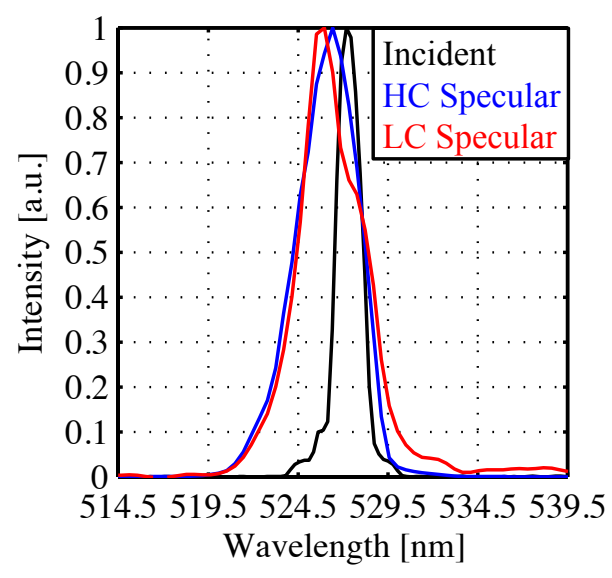

Figure 2.13: (Color) Temporally and spatially integrated spectra of the incident pulse (black), low-contrast specular (red) and high-contrast (blue) specular pulses.

The time integrated, far-field distribution of the low-contrast specularly reflected pulse is shown in Figure 2.14. Subplot (a) is the spatially resolved signal where the 3 circular holes were used to send light to other diagnostics (in particular, the hole closest to the center of the beam was used to send light to the FROG). After subtracting out the hard hits and background, the signal is integrated (interpolating over the holes) and converted into energy 
with the in situ calibration, spectral response of the filters and spectral measurements. The spatial profile of the reflected pulse is quite smooth and only $14.6 \pm 2.5 \%$ of the incident pulse energy captured on the screen. ${ }^{13}$

Also shown are the horizontal (red) and vertical (black) lineouts through the peak ${ }^{14}$ in (b) spatial and (c) spectral domains. ${ }^{15}$ The spot is observed to be asymmetric, being approximately $40 \%$ broader along the horizontal axis as compared to the vertical. Since the incident laser pulse effectively has a 'tophat' distribution (i.e. a circular beam with nearly uniform intensity), the size of the incident laser upon reflection off a perfect mirror target at the scattering plate plane would be approximately $14.1 \mathrm{~cm}$ in diameter, indicated by the vertical dashed black lines in subplot (b).

Similar to the low-contrast shot, Figure 2.15 the specular reflectivity data from the highcontrast shot. Not only is the specular reflectivity nearly $3 \times$ higher than the low-contrast show at $41.6 \pm 8.3 \%$, the spatial intensity distribution is significantly 'spikier'. The specular pulse is again asymmetrically broadened, but this time it is broader along the vertical axis.

Although not directly confirmed with this experiment, a subsequent 'follow-up' experiment confirmed that the asymmetric broadening in the high-contrast regime is consistent with the 'grating' orientation of the rough targets discussed in Chapter 2.4. Figure 2.16(a) shows AFM measurements of an optically flat target (first row) and one of the electroplated targets (second row) like those described previously, oriented consistently with how they were aligned in the target chamber as seen from the incident laser propagation axis. Subplot (b) shows the Spectralon ${ }^{\circledR}$ data obtained from a coaxially aligned sub-aperture CW alignment beam $(f / 12)$. From the optically flat target, we observe a mirror like reflection. The electroplated target, however, exhibits a very obvious broadening but only along the vertical axis. The orientation of the target and broadening direction are consistent with a grating-like reflection (i.e. diffraction pattern) from the horizontally aligned grooves like

\footnotetext{
${ }^{13}$ The energy error bar was obtained by quadrature addition of errors from background subtraction, interpretation over the holes and spectral calibrations [129].

${ }^{14}$ The lineouts are taken as close as possible to the peak without crossing a hole.

${ }^{15}$ The $\sim 500 \mu \mathrm{m} /$ pixel resolution over the $25.4 \mathrm{~cm}$ window resulted in observable spectral modes below $\sim 7 \mathrm{mrad} / \mu \mathrm{m}$.
} 

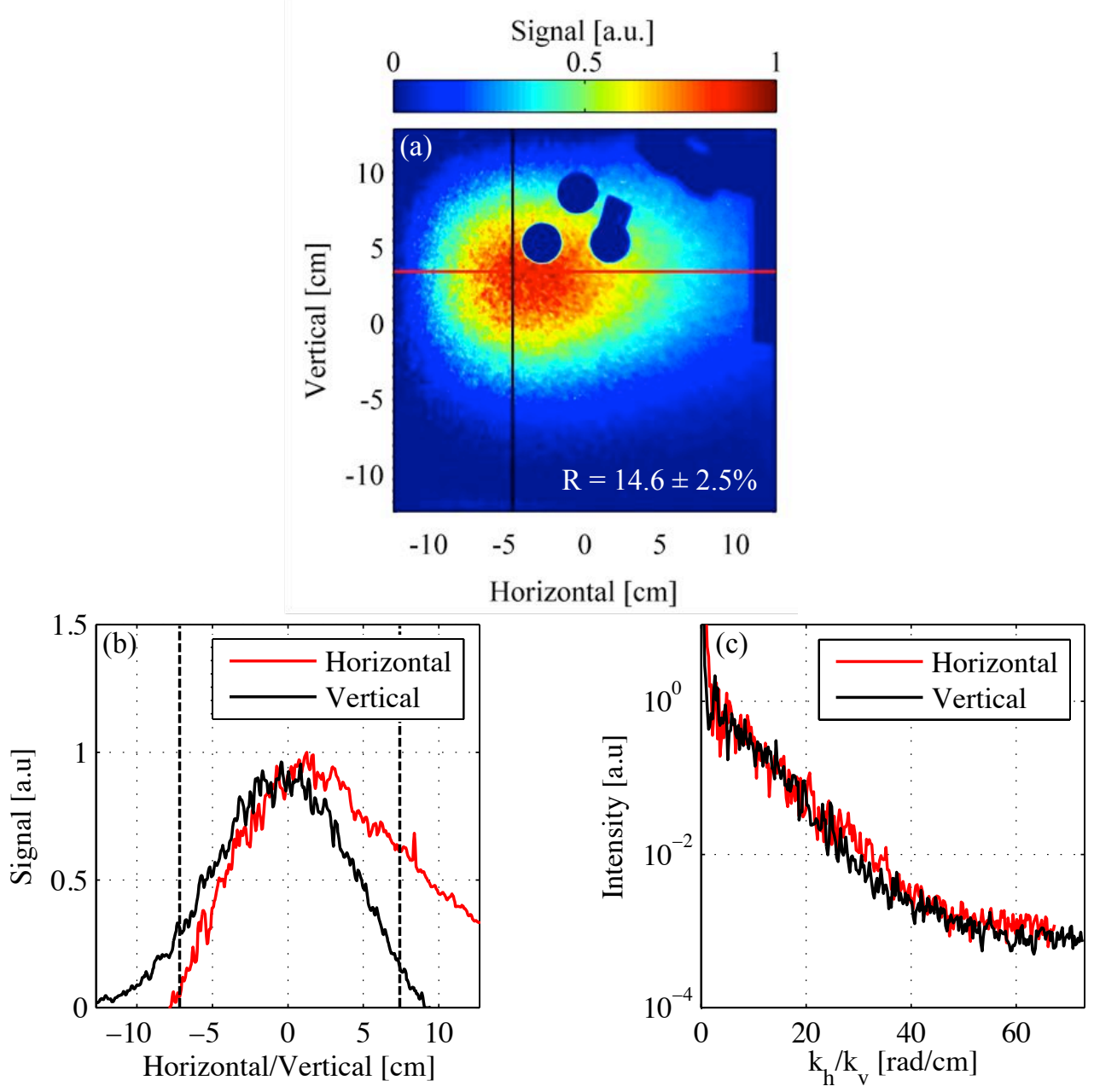

Figure 2.14: (Color) Low contrast specular reflectivity. (a) Time integrated spatial profile of the specularly reflected pulse. (b) Horizontal (red) and vertical (black) lineouts, with the incident beam aperture indicated by the vertical dashed black lines, and (c) corresponding spectra.

our target.

Subplot (c) shows the on-shot specular reflectivity data from the high-contrast short pulse. Again, in the optically flat case, we see a mirror like reflection of the 'tophat' laser profile with more than $80 \%$ reflectivity. ${ }^{16}$ In the rough target case, we again see the same preferential broadening of the specular pulse along a direction consistent with the groove

\footnotetext{
${ }^{16}$ The 'Pac-Man' like intensity distribution is due to an imperfection in the amplification process in one quadrant of the beam.
} 

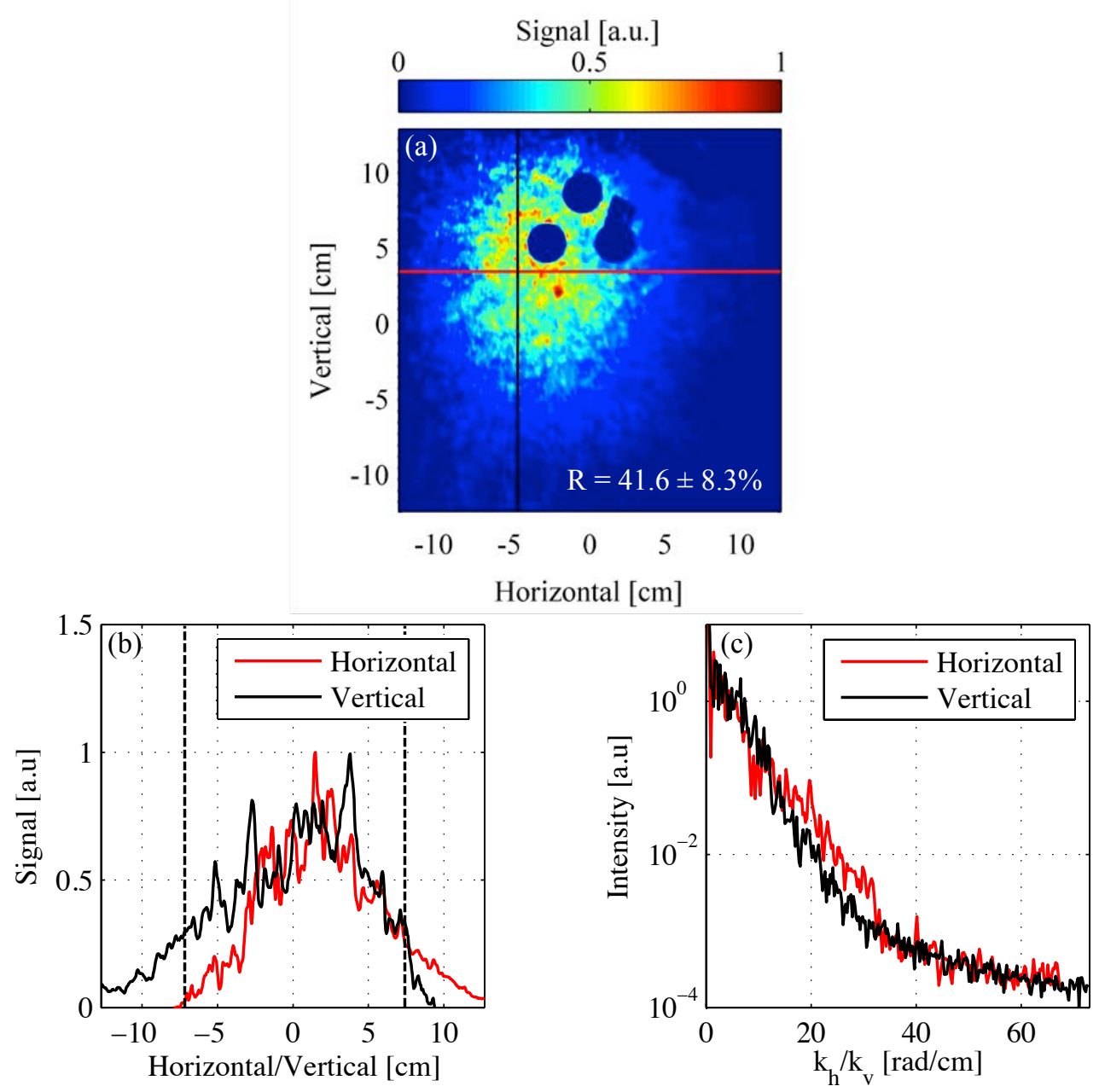

Figure 2.15: (Color) High contrast specular reflectivity. (a) Time integrated spatial profile of the specularly reflected pulse. (b) Horizontal (red) and vertical (black) lineouts, with the incident beam aperture indicated by the vertical dashed black lines, and (c) corresponding spectra.

orientation but with only half the reflectivity. The asymmetric broadening was found to consistently be along the axis perpendicular the groove orientation, indicating that these structures survive long enough throughout the interaction to significantly affect the time integrated spatial profile and energy of the specularly reflected pulse. 
(a) Target AFM

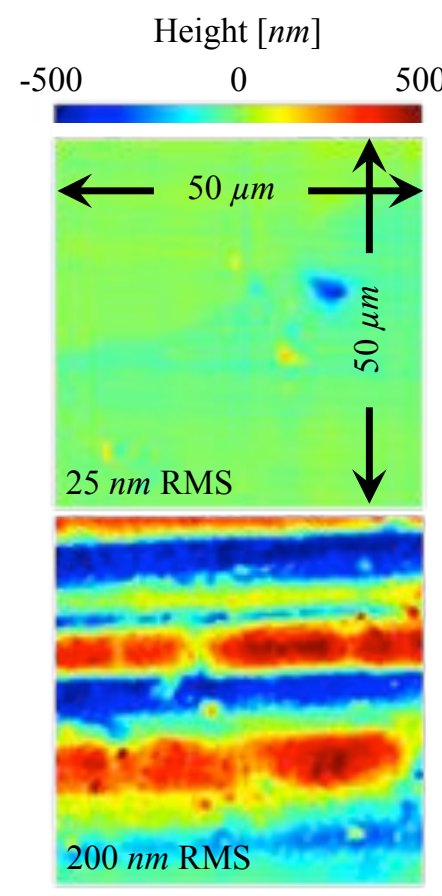

(b) Specular CW (f/12)

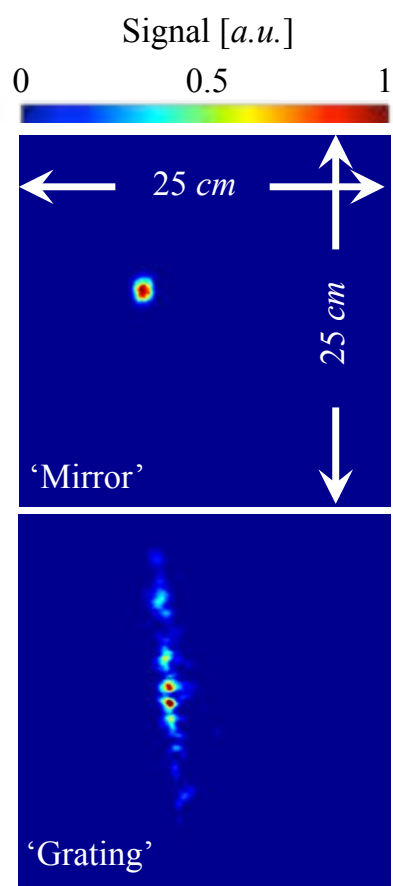

(c) Specular SP (f/2.5)

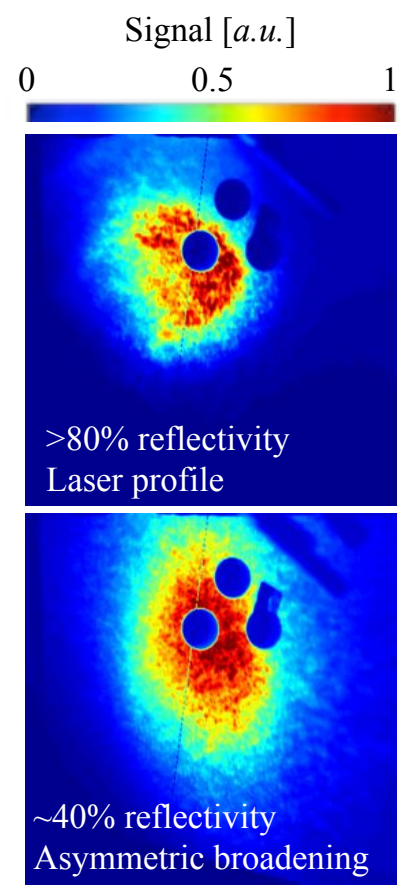

Figure 2.16: (Color) High contrast reflectivity data from an optically flat (top row) and electroplated (bottom row) aluminum targets. (a) Target atomic force microscope measurements of the target. (b) Specular reflectivity off target using coaxially aligned sub-aperture CW alignment laser and (c) the high-contrast short pulse. The optically flat target had nearly perfect reflectivity with the incident laser spatial profile reflected where the rougher target still exhibits asymmetric broadening consistent with a grating-like diffraction pattern (horizontal grooves) but with only half the reflectivity of the optically flat target.

\subsubsection{Specular FROG measurements}

The hole in the Spectralon ${ }^{\circledR}$ closest to the center of the specular beam was used to send light to another PG FROG to obtain the temporally resolved intensity and instantaneous wavelength shift of the specularly reflected pulse. The down collimating lens pair (labeled as $L_{1}$ and $L_{2}$ in Figure 2.4) consisted of a positive $f=+50 \mathrm{~cm}$, 2" diameter lens $\left(L_{1}\right)$ $\approx 58 \mathrm{~cm}$ away from TCC and a negative $f=-60 \mathrm{~cm}, 2$ " diameter lens $\left(L_{2}\right) \approx 256 \mathrm{~cm}$ away from $L_{1}$. These optics collected approximately an $f / 50$ beam out of the $\sim f / 2.5$ specular pulse and collimated it (requirement for the diagnostic) down to approximately 
$1 \mathrm{~cm}$ in diameter before being directed into the FROG. Higher harmonics generated in the interaction were blocked from entering the FROG with a piece of $K G 3$ [131].

The low-contrast FROG data is shown in Figure 2.17(a) as the square of the intensity to illuminate the low intensity portions of the signal. Since the PG FROG trace has an asymmetry in delay, the chirp in the pulse can be directly inferred from the spectrogram; clearly a large redshift occurs at early times in the pulse but quickly decays by the peak of the pulse. The retrieval with the best (minimal) FROG error is shown in subplots (b)-(d) where the best error for the $1024 \times 1024$ grid was around $2 \%$. Compared to the incident pulse, the specular pulse would appear to have much more structure and looks significantly longer in duration and broader in spectrum.

The phase retrieval algorithm was run 100 times with different initial seeds to obtain a measure of the consistency of the pulse reconstruction. These results are shown in Figure 2.18 with the (a) time domain and (b) wavelength domain representations. Large standard deviation error bars (gray bands, $\gtrsim 25 \%$ ) indicate that the exact structure of the intensity profile is not well constrained in the retrieval. On average, the pulse duration was longer with approximately a $1.3 \pm 0.3 \mathrm{ps}$ FWHM. The instantaneous wavelengths, as we had speculated from the raw FROG trace, start off redshifted at early times (peaked at $\Delta_{\lambda} \approx+3.2 \pm 1 \%$, $2 p s$ before the peak of the specular pulse), shift back to the carrier by the peak of the pulse and eventually end in a blueshift $(\approx-0.8 \pm 0.2 \%)$ on the trailing edge of the pulse. The averaged reconstructed spectral intensity from the FROG measurement is shown in subplot (b) with a $3.6 \pm 0.1 \mathrm{~nm}$ FWHM centered around $526 \mathrm{~nm}$. The independent measurement of the spatially averaged spectrum is also shown (blue). While they differ slightly, both spectra do show similar trends of increased redshift contributions. This discrepancy is likely due to the FROG only sampling a small spatial portion of the pulse around the specular axis whereas the integrated spectrum summed over the entire distribution. Unfortunately, a separate spectrum of what the PG FROG sampled was not obtained.

The high-contrast FROG trace is shown in Figure 2.19(a). The retrieval with the best (minimal) FROG error is shown in subplots (b)-(d) where the best error for the $1024 \times 1024$ grid was around $1.6 \%$. Just like the low-contrast shot, the specular pulse would appear to 

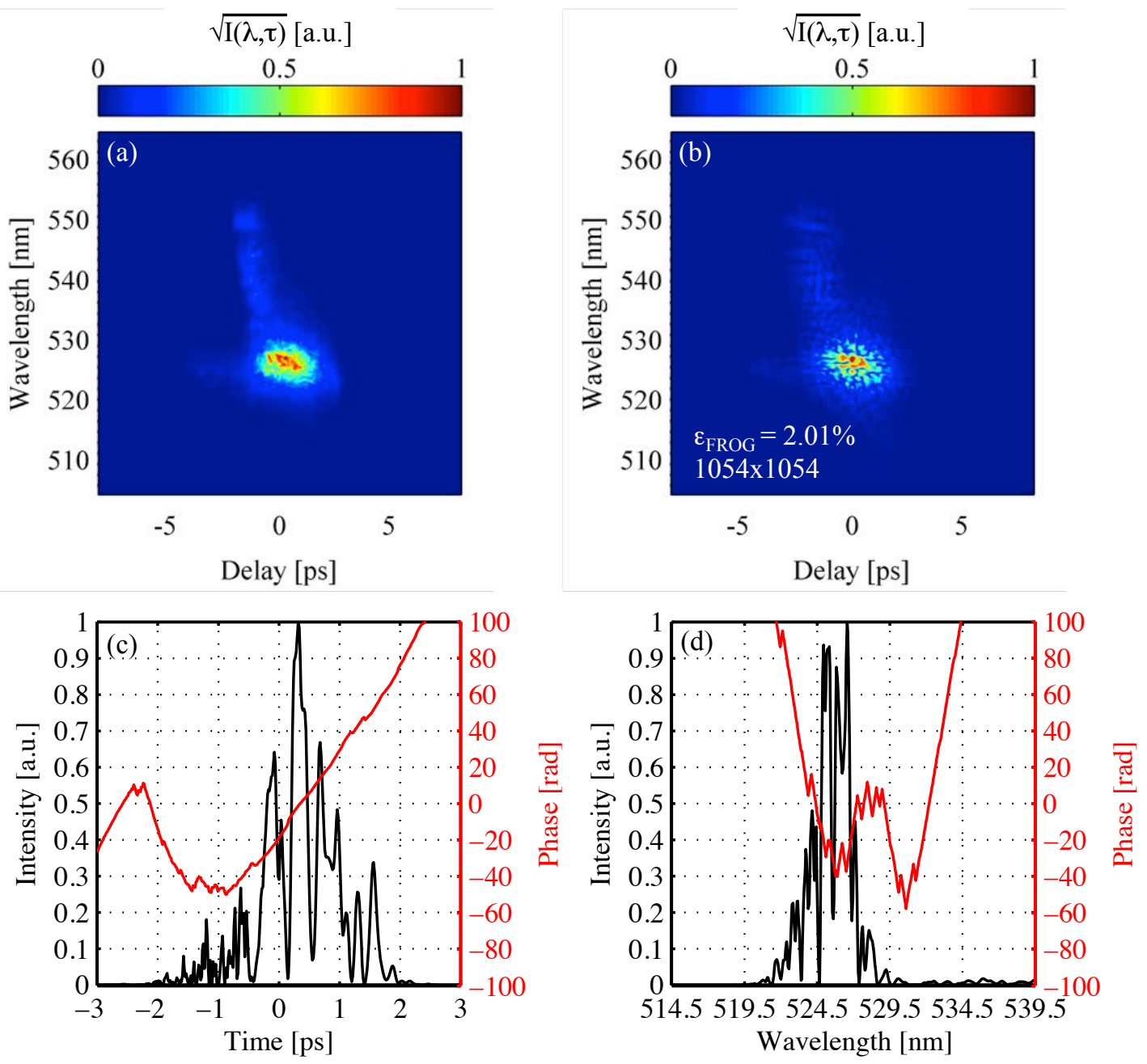

Figure 2.17: (Color) Low contrast specular FROG trace. Subplot (a) is the experimental data, shown as the square root of the signal to emphasize low energy portions of the trace. Subplot (b) is the best retrieved trace out of 100 reconstructions with subplot (c) showing the temporal intensity and phase profile and $(\mathrm{d})$ the spectral profile.

have much more structure than the incident pulse.

Once again, the phase retrieval algorithm was run 100 times with different initial seeds to obtain a consistency of the reconstructed pulse structure. These results are shown in Figure 2.20 with the (a) time domain and (b) wavelength domain representations. We find a much smoother pulse profile after averaging over these 100 traces but larger standard deviation error bars (gray bands, $\gtrsim 25 \%$ ) indicate that the exact structure of the intensity profile 

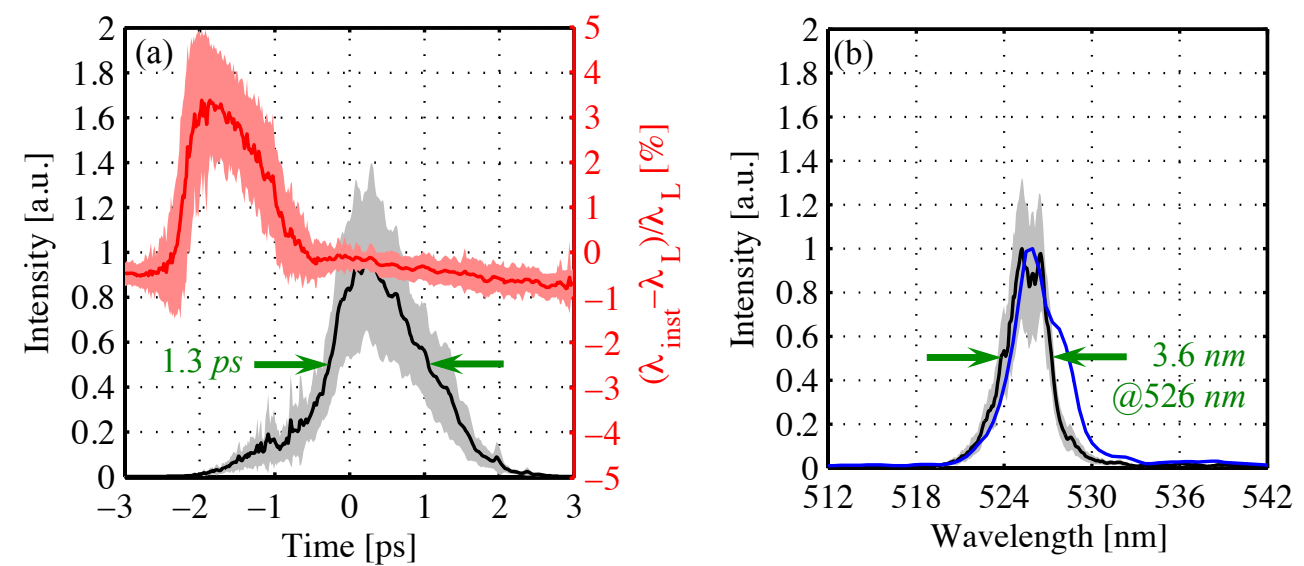

Figure 2.18: (Color) Low contrast specular FROG trace. Subplot (a) contains the instantaneous intensity (black) and wavelength (red) and subplot (b) contains the average spectral intensity (black) and an independent spectrum measurement (blue). These curves are the error weighted average of 100 separate retrievals and the error bars (shaded gray area for intensity, red error bars for instantaneous wavelength shift) are $\pm \sigma_{S T D}$ about the mean.

is not well constrained in the retrieval. On average, the pulse duration was longer with approximately a $700 f s$ FWHM but now with lower intensity 'wings' preceding and trailing the peak visible. Either these wings were present in the laser trace but not visible because of the dynamic range of the instrument or the pulse has somehow gotten longer through the interaction. Also shown is the instantaneous wavelength shift (in red). The instantaneous wavelengths are overall 'bluer' than the incident laser pulse with $\Delta_{\lambda}$ ranging between -1 and $0 \%$ of $\lambda_{L}=527 \mathrm{~nm}$. Fortunately, despite the uncertainty in the structure of the intensity profile, the error bars on the phase are small enough to not drive much uncertainty in the instantaneous wavelength measurement (being no larger than $\pm 0.2 \%$ ). Similarly, the wavelength domain is shown in subplot (b) where the pulse has a $3.1 \mathrm{~nm}$ FWHM centered around $526 \mathrm{~nm}$. Also shown is the spatially integrated spectrum measurement (blue) from Figure 2.13. Although similar, they differ beyond the error bars of the reconstruction. 

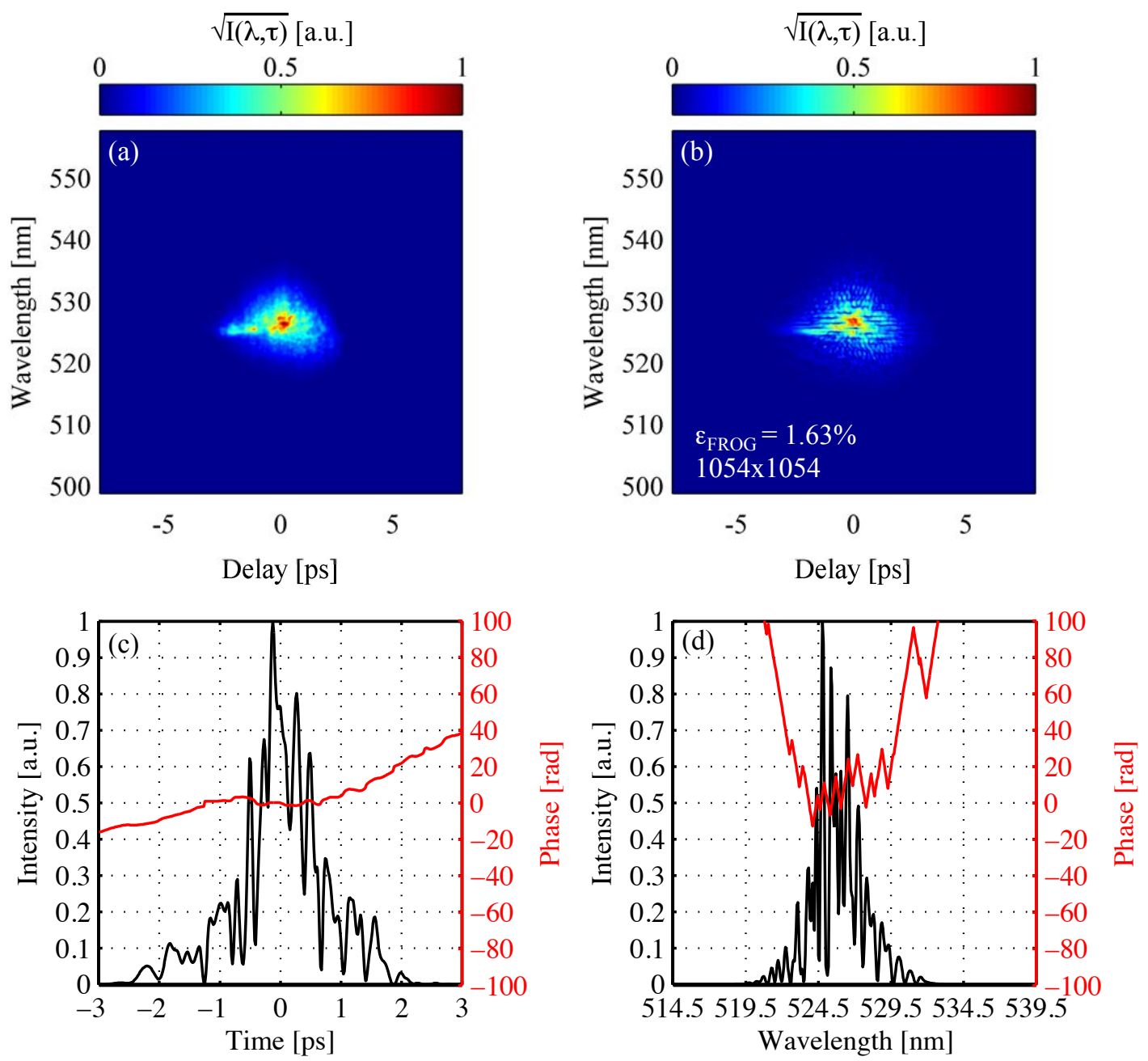

Figure 2.19: (Color) High contrast specular FROG trace. Subplot (a) is the experimental data, shown as the square root of the signal to emphasize low energy portions of the trace. Subplot (b) is the best retrieved trace out of 100 reconstructions with subplot (c) showing the temporal intensity and phase profile and $(\mathrm{d})$ the spectral profile.

\subsection{Summary}

In summary, we performed an experiment that studied the effects of under-dense pre-plasma on specular reflectivity and coupling into hot-electrons. The results of the experiment are summarized in Table 2.3.

While ultra-intense short-pulse lasers are typically preceded by several $m J s$ or more 

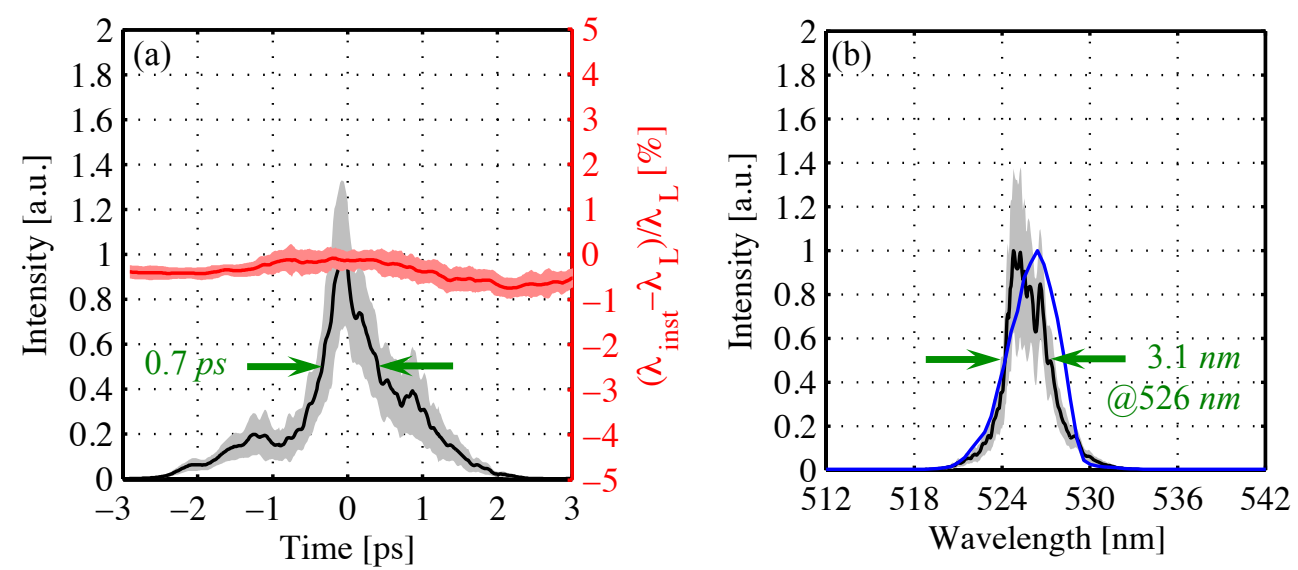

Figure 2.20: (Color) High contrast specular FROG trace. Subplot (a) contains the instantaneous intensity (black) and wavelength (red) and subplot (b) contains the average spectral intensity (black) and an independent spectrum measurement (blue). These curves are the error weighted average of 100 separate retrievals and the error bars (shaded gray area for intensity, red error bars for instantaneous wavelength shift) are $\pm \sigma_{S T D}$ about the mean.

\begin{tabular}{cccc}
\hline \hline Diagnostic & Laser & High Contrast Specular & Low Contrast Specular \\
\hline \hline Pre-pulse & - & $<10 \mu J, 3 \mathrm{~ns}$ & $3.3 \mathrm{~mJ}, 3 \mathrm{~ns}$ \\
\hline \multirow{5}{*}{ FROG } & $0.46 \mathrm{ps}$ FWHM & $0.7 \mathrm{ps}$ FWHM & $1.3 \mathrm{ps}$ FWHM \\
& $\lambda_{o}=527 \mathrm{~nm}$ FWHM & $3.1 \mathrm{~nm} \mathrm{FWHM}$ & $3.6 \mathrm{~nm}$ FWHM \\
& $\left|\Delta_{\lambda}\right|<0.25 \%$ & $\lambda_{o}=526 \mathrm{~nm}$ & $\lambda_{o}=526 \mathrm{~nm}$ \\
& $-1 \%<\Delta_{\lambda}<0 \%$ & $3.0 \pm 1 \%$ early redshift \\
\multirow{2}{*}{ Spectra } & $1.7 \mathrm{~nm} \mathrm{FWHM}$ & $4.1 \mathrm{~nm} \mathrm{FWHM}$ & $0.8 \pm 0.2 \%$ late blueshift \\
& $\lambda_{o}=527 \mathrm{~nm}$ & $\lambda_{o}=526 \mathrm{~nm}$ & $\lambda_{o}=526 \mathrm{~nm}$ \\
\hline \multirow{3}{*}{ Reflectivity } & $(\sim 35 J)$ & $41.6 \pm 8.3 \%$ & $14.6 \pm 2.5 \%$ \\
& - & very speckled pattern & smoother pattern \\
& - & asymmetric broadening & asymmetric broadening \\
\hline \hline
\end{tabular}

Table 2.3: Summary of specular pulse properties, broken up by diagnostic, observed in the experiment.

of laser energy nanoseconds before the main pulse arrives at the target, our intrinsic prepulse levels were found to be less than $10 \mu \mathrm{J}$. For these shot conditions, initial target 
conditions become extremely important and significant enhancements in absorption using targets with initial surface perturbations (over perfectly flat interfaces) have been reported. These enhancements have been attributed to enhanced Brunel [72, 98], resonant excitation of surface plasma waves [117-120] or local field enhancement via Mie resonance [121, 122] depending on the specifics of each study. Without simulations, it is a priori difficult to surmise which of these effects (if any) are dominant for our targets of interest or which scale surface perturbations affect the various observations in the specular pulse properties.

The targets shot during the experiment had a large range of surface perturbations in both RMS and spectra, the specifics of which depended largely on the $C u$ fluor depth. From the reflectivity data, the most laser energy that could be coupled into electrons for the highcontrast shot conditions was $\lesssim 58 \%$ (which doesn't even take into account scattered light, harmonic generation, target heating or quasi-static field energy in the target which would all detract from this number). We also know that the reflected spectra has relatively small instantaneous wavelength shifts and integrated spectrum with an overall $1 \mathrm{~nm}$ blueshift. While many mechanisms can cause spectral shifts, it is likely that the dominant effect is the ionization blue shift as there is very little pre-plasma. Preferential broadening direction in the spatial profiles of the specular pulse indicate that the grating-like surface features survive, at least to some degree, throughout the interaction.

Since the dynamic competition between heated plasma expansion and laser profile steepening can drive changes in the electron density profile, it is likely that these features will not make a large difference for lasers with significant pre-pulse. Plasma expansion that cannot be overcome by the laser profile steepening can lead to smoothing over of the absorption enhancing perturbations by the motion of the relativistic critical density surface and the development of an under-dense plasma shelf though which the laser must propagate. The extent to which absorption in this high-contrast pulse interaction transitions from being dominated by sharp interface absorption mechanisms to under-dense pre-plasma phenomenon will be addressed using particle-in-cell (PIC) simulations.

Controllable pre-pulse was injected using a coaxially aligned long-pulse resulting in $3 \mathrm{~mJ}$ of energy delivered in the $3 \mathrm{~ns}$ before the short-pulse. From radiation hydrodynamic 
calculation with MULTI2D, $10 s$ of microns of under-dense plasma was created in front of the target before the main pulse arrived. Not only does this pre-plasma move the laser interaction interface from supra-critical solid density to the lower relativistic critical density, it also subjects the main pulse to instabilities [47, 58-60, 132]

Previous work by Yuan Ping et al [35] has shown that the early red shift in instantaneous wavelength on the rising edge of the specularly reflected pulse directly corresponds to the Doppler shift that arises from the motion of the critical surface. Their experimental traces, obtained from low-contrast pulse interactions, showed that the redshift was largest at the beginning of the pulse at nearly $6 \%$, gradually dropped to $0 \%$ shortly after the reflection of peak of the pulse and ended with a $1.5 \%$ blueshift. The early redshift was found to correspond to a $3 \%$ the speed of light and was corroborated using PIC simulations with experimentally realistic conditions. The late blueshift corresponds to a reversal of the motion, indicating heated plasma expansion toward the laser. However, this work was found to be insensitive to dynamic ionization and relativistic self-phase modulation which may not be the case for our experiment.

Other simulation and experimental studies in this regime have shown electron source energy spectrum scaling with $I \lambda^{2}$, commonly referred to as Ponderomotive scaling [34]. Increasingly stronger contributions from collisionless absorption mechanisms with ultrarelativistic, low-contrast pulses have also been experimentally observed [90] as well as simulations that suggest angular spectrum broadening of the hot-electron source with increasing pre-plasma scale length $[57,133]$. However, quasi-static field generation in the under-dense plasma $[45,46]$ has been shown to greatly modify relativistic electron generation and transport $[72,89]$. 


\section{Chapter 3}

\section{THE SHAPED CRITICAL SURFACE IN LOW-CONTRAST HIGH-INTENSITY LASER PLASMA INTERACTIONS}

As previously discussed, extensive under-dense preformed plasma can develop around the laser interaction region before the main pulse arrives due to pre-pulse in low-contrast interactions. This pre-plasma, due to a variety of non-linear phenomena, can drastically modify the incident laser pulse as it propagates through the plasma before ultimately being reflected at the relativistic critical surface. In this Chapter, which was previously published as The Shaped Critical Surface In High Intensity Laser Plasma Interactions [Physics of Plasmas, 18(1):013102, 2011] [78], we ${ }^{17}$ use simulations to show how the far-field spatial intensity distribution of the specularly reflected light is influenced by the curved shape of the relativistic critical surface and surrounding pre-plasma. Additionally, we show how it can provide a simple and meaningful diagnostic for determining the pre-plasma electron density profile near the critical surface in low-contrast relativistic laser-plasma interactions, a region that is notoriously difficult to probe experimentally.

\subsection{Abstract}

We investigate the properties of the relativistic critical surface in a high intensity laserplasma interaction, specifically the spatial morphology of the surface and its effects upon

\footnotetext{
${ }^{17}$ I provided support for simulations, analysis and analytic model development but the article was penned by D. W. Schumacher. Few modifications of the original text have been made with the exception of a more inclusive model description.
} 
the divergence of the reflected light. Using the commercial, particle-in-cell code LsP running in two dimensions $(2 \mathrm{D} 3 \mathrm{~V})$, we model the formation of the critical surface and show that it resides at a varying depth into the material that is dependent upon both the intensity radial dependence of the laser focus as well as the shape of the longitudinal vacuum-material interface. The result is a shaped "mirror" surface that creates a reflected beam with phase and amplitude information informed by the extent of the pre-plasma present before the intense laser pulse arrived. In effect, we propose a robust, highly effective means of experimentally determining the pre-plasma conditions for any high intensity laser-mater interaction. We elucidate the important physics with a simplified model that, within reasonable intensity bounds, recasts the effect of the complex laser-plasma interaction on the reflected beam into a standard Gaussian optics calculation.

\subsection{Introduction}

High intensity laser-solid matter interactions generally begin as an interaction between the laser and plasma at densities well below $1 \mathrm{~g} / \mathrm{cm}^{3}$, at the critical surface. This is the case even for targets with initially sharp vacuum material interfaces because the peak intensity of the laser pulse is usually preceded by lower intensities of sufficient duration that, before the peak of the laser pulse arrives, the front of the target is well ionized yielding an interface region of plasma, often called pre-plasma. The critical surface is defined as the region where the laser frequency equals the local, intensity dependent, plasma frequency, and occurs at the critical

electron density $n_{c}=\left(\gamma m_{e} \omega_{L}\right)^{2} /\left(4 \pi e^{2}\right)(\mathrm{cgs})$, where the relativistic factor $\gamma=\sqrt{1+a_{o}^{2} / 2}$ [85]. Here, $a_{0}=e E /\left(m_{e} \omega_{L} c\right),-e$ and $m_{e}$ are the electron charge and mass, and $E$ and $\omega_{L}$ are the laser field amplitude and angular frequency, respectively. The relativistic factor becomes significant when $a_{0} \geq 1$ or the intensity exceeds roughly $10^{18} \mathrm{~W} / \mathrm{cm}^{2}$. For a $1 \mu \mathrm{m}$ wavelength, low intensity laser, the critical density is about $1.1 \times 10^{21} \mathrm{~cm}^{-3}$. In a focused laser beam the intensity profile is a strongly decreasing function of the radial distance from the focal spot center, so the most intense portion of the laser spatial profile requires a higher plasma density before it will interact and thus penetrates farthest into the pre- 
plasma. This is the regime of self-induced transparency [134]. The resulting shaped critical surface causes the region of strong laser interaction to be spread out along the pre-plasma density gradient. Experiments do not generally measure the pre-plasma under conditions of interest because no simple nor particularly effective method exists to do so. Shadow and interferometric techniques provide useful information, but only for densities below that of the non-relativistic $(\gamma=1)$ critical surface [134, 135] X-ray probes have also been used, but lack resolution [136]. Pirozhkov, et al. have recently shown that the measurement of the reflectivity of a high intensity laser beam from the target can be reliably used to indicate whether a significant pre-plasma is present; however they describe no means of determining the actual pre-plasma density [137]. Here we use numerical simulations and a remarkably simple model to predict that a given shaped critical surface - which we show depends directly upon the laser and pre-plasma spatial profiles - will have an easily obtained experimental signature that can be used to infer the properties of the pre-plasma at the densities relevant in an experiment. Although this present work is strictly valid in the limit where relativistic plasma self-focusing can be strong, but not so strong as to create a large number of filaments, we show that these results can be used to characterize pre-plasma conditions in general.

In Chapter 3.3 we describe our particle-in-cell (PIC) simulations including the algorithms employed, relevant numerical parameters, and representation of the target. We describe the formation of the shaped critical surface and its effect on the laser in Chapter 3.4. We also describe how key aspects of the generally complicated propagation of an intense laser can be treated with a simple model based on standard, linear Gaussian optics. In Chapter 3.5 we show the results of our PIC simulations and compare to the results of our model. The effects of mechanisms outside our model are discussed. Finally, in Chapter 3.6, we propose a new pre-plasma diagnostic based on our results and summarize. 


\subsection{PIC simulations}

We have employed the commercial, particle-in-cell code LSP [138] which solves the Maxwell curl equations and the Lorentz force equation on a discrete spatial grid. We represented a $C u$ plasma using up to 100 million particles in two Cartesian dimensions $(X Z)$. The simulations employed fully mobile electrons and $C u+$ ions, 15 grid cells per wavelength, and 60 time steps per optical cycle, so the skin depth and associated absorption/reflection mechanisms were resolved. Although collisions are not especially important for this problem, we employed the Jones algorithm [139] using the Spitzer collision rate nevertheless. Our results are robust against changes in all aspects of the simulation, including choice of ion and ionization state. (We note that LSP is generally free of numerical heating in this regime.) Figure 3.1 shows a typical simulation geometry. The plasma and pre-plasma are in the middle of the grid with a vacuum layer surrounding it. The plasma is solid density $\left(n_{\text {solid }}=8.5 \times 10^{22} \mathrm{~cm}^{-3}, \approx 77 n_{c}\right)$ with an initial temperature of $100 \mathrm{eV}, 15 \mu \mathrm{m}$ thick and $75 \mu \mathrm{m}$ wide. A short laser pulse is injected from the left traveling towards the plasma (in the $+x$ direction); the pulse had a $1 \mu \mathrm{m}$ wavelength, a trapezoidal envelope in time (130 $\mathrm{fs}$ duration with linear $20 \mathrm{fs}$ rise and fall times), and a Gaussian spatial profile (at the waist, $w_{o}=5 \mu m$ ). The pulse was focused to have a waist at $x=0 \mu m$ in vacuum (see Figure 3.1). The linearly polarized electric field is in the plane of the simulation and the magnetic field is normal to it with the laser incident normally on the target. The pre-plasma was specified as having an exponentially varying density profile $\left(n_{e}=n_{\text {solid }} \exp (x / L), x<0\right)$, where $L$ is the pre-plasma scale length. The pre-plasma starts at $x=-10 L$ and extends to the solid density boundary at $x=0$. The initial pre-plasma density is roughly $10^{-5} n_{\text {solid }}$.

\subsection{The shaped critical surface and a simple model}

Figure 3.2 shows the pulse at the time the peak of the pulse is reflecting for $L=1 \mu \mathrm{m}$ and $3 \mu \mathrm{m}$ pre-plasmas by plotting the y-component of the magnetic field on a log scale. We specify the pulse peak intensity, $I_{p}$, by the value it would have had at the waist in vacuum,

in this case $I_{p}=10^{19} \mathrm{~W} / \mathrm{cm}^{2}$. The actual peak intensity is higher at the critical surface 


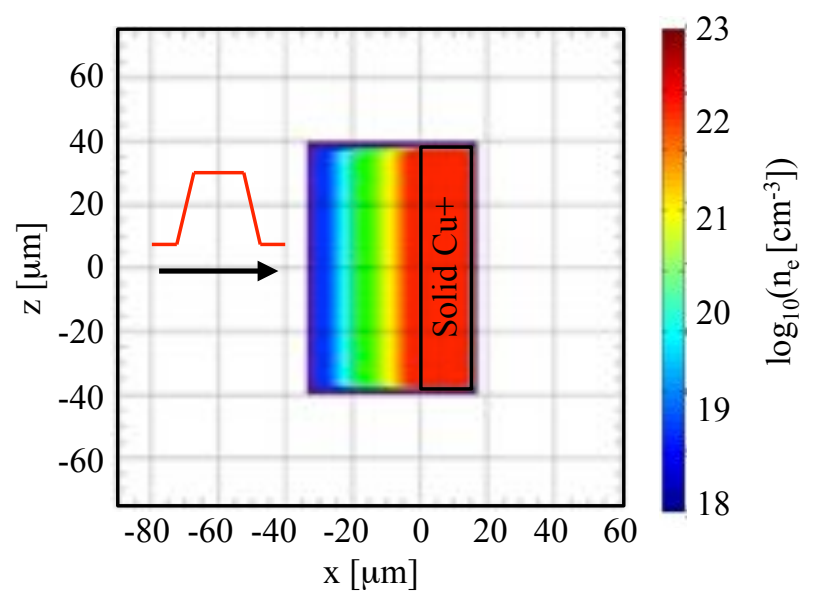

Figure 3.1: (Color) Simulation geometry with the pulse incident from the left. The target consists of solid density copper plasma extending between $x=0$ and $15 \mu \mathrm{m}$ (red), with $L=3 \mu m$ pre-plasma at the interface (rainbow).

due to reflection of the light that is not absorbed and self-focusing. Absorption is known to be roughly $50 \%$ for the conditions treated here, with much of the absorbed energy going into the generation of relativistic electrons [90]. We note the pulse has broken into a small number of filaments. The critical surface is also plotted on the figure for $I_{p}$ and $2 I_{p}$. In the nonrelativistic limit the critical surface would be flat, but here it is clearly deformed; dramatically so for the $3 \mu \mathrm{m}$ case. The critical surface is at a minimum plasma density (nonrelativistic limit) for the wings of the pulse where the field is weak, but bends towards higher density where the field of the laser pulse is large. Noting that the pre-plasma density gradient is $\sim L^{-1}$, the larger the spatial extent of the plasma, the more the critical surface will deform along the laser propagation direction. Here lies the key point: it is the critical surface that defines the surface off which the laser pulse reflects. By analyzing the reflected light directly we record information about the deformation of the critical surface and thus the scale-length, $L$, of the pre-plasma. The reflected light comes from exactly that part of the interaction of greatest interest for understanding the laser-plasma interaction; a region that is opaque to alternate methods employing shadowing or interferometry.

What is, in principle, a powerful analytic diagnostic for pre-plasma determination is 

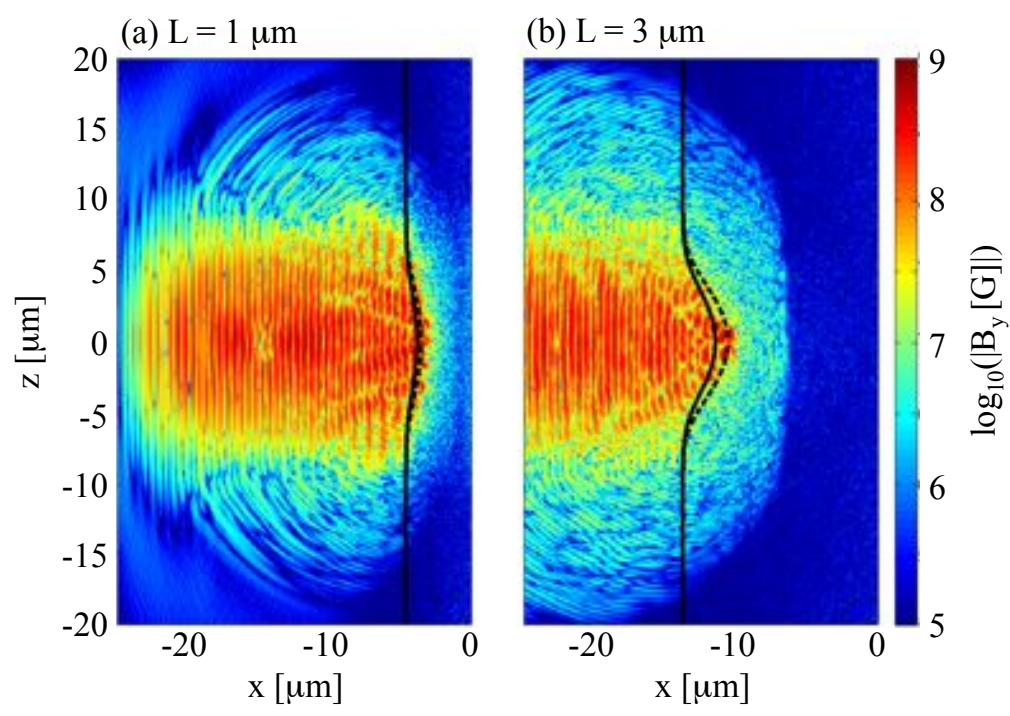

Figure 3.2: (Color) Magnetic field $\left(B_{y}\right)$ at the time the peak of the pulse reflects for (a) $L=1 \mu \mathrm{m}$ and (b) $L=3 \mu \mathrm{m}$ scale length pre-plasmas. The solid (dashed) black line follows the calculated critical surface for $I_{p}=10^{19} \mathrm{~W} / \mathrm{cm}^{2}\left(2 \times 10^{19} \mathrm{~W} / \mathrm{cm}^{2}\right)$.

bounded in its usefulness by the necessity of rather significant modeling resources. We have addressed this issue by proposing a simple model that abandons almost all details of the light-plasma interaction, yet still permits quantitative prediction and, most importantly, provides an intuitive guide. The critical surface has a complex transverse profile (depicted in Figure 3.2) which is reasonably fit by the analytic expression (black) given by Equation 3.1 for this simple initial electron density and laser geometry. The radial region extending from $z=-w_{o}$ to $w_{o}$ is reasonably fit by a parabola $\left(x=x_{o}-a z^{2}\right)$ and defines an effective parabolic mirror, focal length $f_{M}=1 /(4 a)$, from which the light reflects.

$$
x_{c}(z)=L \ln \left[\frac{m_{e} \omega_{L}^{2}}{4 \pi n_{\text {solid }} e^{2}} \sqrt{1+\frac{4 \pi e^{2}}{m_{e}^{2} \omega_{L}^{2} c^{3}} I(z)}\right]
$$

To reach the shaped critical surface, the laser phase fronts must travel through different thicknesses of pre-plasma, as illustrated by the cross-hatched region in Figure 3.3(a). This region of pre-plasma forms an effective lens (Figure 3.3(b)) but, since the index of refraction 
(a)

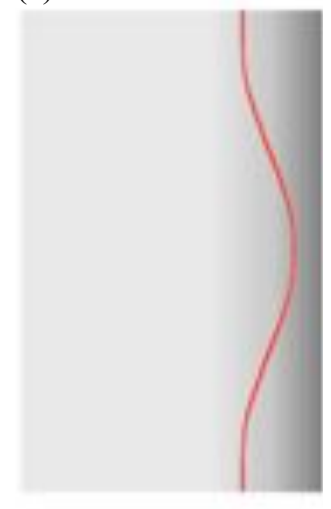

(b)

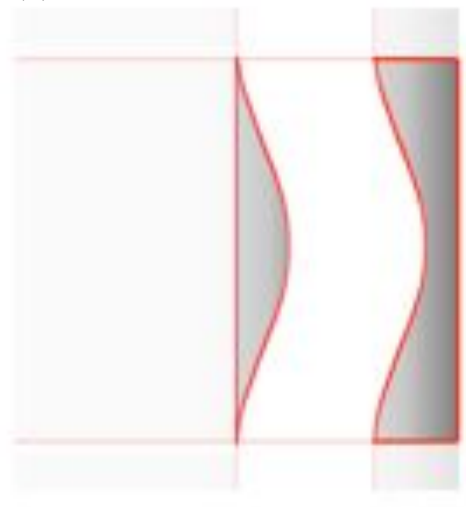

(c)

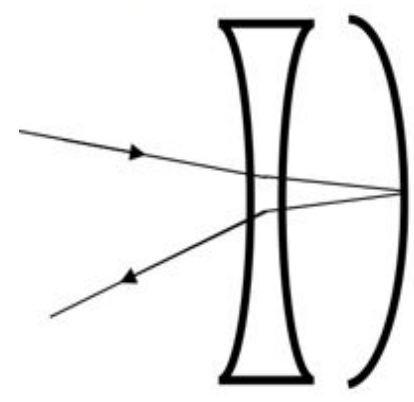

Figure 3.3: (Color) (a) Cartoon depiction illustrating the shaped critical surface (red line) and the pre-plasma (shaded gray). (b) The optical components of the lensing system: the portion of the pre-plasma bounded by the critical surface (left) and the approximately parabolic shape of the relativistic critical surface (right). (c) The equivalent optical system as seen by the laser pulse where the bound plasma becomes a negative lens.

is less than unity in a plasma, the lens is diverging. Thus, in order to reflect, the intense pulse effectively passes through a diverging lens, reflects from a converging mirror, and then passes back through the lens; a process depicted in Figure 3.3(c). The focal length of the lens is given approximately by the Lens Makers formula, $1 / f_{L}=(\eta-1) / R$, where $\eta$ is the index of refraction of the pre-plasma and $R$ is the radius of curvature characterizing the critical surface, $\left(R \approx 2 f_{M}\right)$. The index of refraction varies due to the density, so we simply evaluate $n$ using the average pre-plasma density in the lens. Finally, the focal length of the combined system, $f$, is given by $1 / f=1 / f_{M}+2 / f_{L}$, since the optical components are close to each other. By including the Gaussian spatial profile of the laser in the expression for the critical density and solving for the $(x, z)$ location of this density given the exponential profile of the pre-plasma, we easily derive an analytical expression for the critical surface and the focal length, $f$. The full expression is algebraically complex, but we find $f \propto w_{o}^{2} / L$. This is a physically appealing result since we expect this effect to become large (small focal length) when the laser is tightly focused in a long scale length pre-plasma. Given $f$, the effect on the reflected light is easily determined using the well-known Gaussian q-parameter: 


$$
\frac{1}{q}=\frac{1}{R}-i \frac{\lambda}{\eta \pi w^{2}}
$$

where $R$ is the radius of curvature of the Gaussian phase front and $w$ the field spot size [140]. The effective lens/mirror/lens formed by the shaped critical surface in the pre-plasma transforms the laser pulse according to: $1 / q_{\text {out }}=1 / q_{\text {in }}-1 / f$. Note the output pulse is completely specified by $q_{\text {out }}$ in this model. For application, we need three input parameters: $L, q_{i n}$, and $I_{p}$. In an experiment $\mathrm{L}$ would be a fitting parameter. Here, we will simply compare results to LSP. We ignore self-focusing and determine $q_{i n}$ by taking $R_{\text {in }}=\infty$ and $w$ to be the waist size, $w_{o}$, in vacuum (this is often measured in experiment). The peak intensity at the critical surface will be somewhere between one and four times the nominal peak intensity, $I_{p}$, depending on how much is reflected. For simplicity, we use $2 I_{p}$.

The effect of the shaped critical-surface can be large. For the conditions of Figure 3.2(b), the effective focal length is approximately $5 \mu \mathrm{m}$, a powerful lens indeed, although acting at a point where the incoming pulse is essentially at its waist. The outgoing beam is predicted to suffer a divergence approximately eight times the incoming divergence. The primary prediction of this model, then, is that there is an increased divergence in the reflected beam relative to the input beam due to the shaped critical surface, with the divergence growing with increasing pre-plasma scale length, increasing intensity or decreasing spot size.

\subsection{Results and discussion}

Clearly the simplified model must generate results that are in agreement with those from the computationally intensive LSP simulation. The LSP simulations were performed on a supercomputer, requiring about a day for the pulse to fully reflect and separate itself by $40 \mu \mathrm{m}$ from the pre-plasma where it could be analyzed. In general, we find the outgoing field is so severely perturbed that an outgoing divergence angle cannot be reliably identified. This presents a practical problem for comparison, either to experimental measurements, or to the simplified model, where the size of the reflected beam in the far field (i.e. after diffraction is fully manifested) is what is measured. Using LSP to model the propagation of the initial 
reflected pulse into the far field would be prohibitively costly both in time and money; instead, we determined the far field reflected pulse by using the near field as determined by LSP and propagating it according to Fresnel diffraction theory [141], discussed in more detail Chapter 4.2.2.

Some typical results are given in Figure 3.4 for three scale lengths: $0.3 \mu \mathrm{m}, 1.0 \mu \mathrm{m}$, and $3.0 \mu \mathrm{m}$. The vacuum pulse peak intensity was $10^{19} \mathrm{~W} / \mathrm{cm}^{2}$. In each case, the pulse spatial profile in the far field for a perfect, flat mirror reflection is given in black. The LSP results after propagation into the far field are given in red and the predictions of the simple model in green. The simple model does a good job of specifying the envelope of the reflected intensity for longer scale lengths, but not for the case of extremely short scale lengths. (We note that in most cases, high intensity, high energy lasers will produce pre-plasmas with $L>1 \mu m[132])$. Taken alone, the PIC simulation is informative but not insightful since it is not at all obvious from inspection of the grid why the reflected pulse diverges. The model, on the other hand, predicts such a divergence and provides a guide to our insight.

The difference between LsP and the model for small $L$ is interesting in its own right. For this case, the normally dominating effects of the shaped critical surface and self-focusing are absent. For very short scale lengths the critical surface is nearly flat and so the reflecting surface is like a planar mirror and will not modify the divergence. Once the pulse reaches densities of $\approx 10^{21} \mathrm{~cm}^{-3}$, the distance required for filamentation at $10^{19} \mathrm{~W} / \mathrm{cm}^{2}$ is roughly $6 \mu \mathrm{m}$, so the effect of self-focusing is also minimal. Finally, we note that fine scale structure is evident in all three graphs in Figure 3.4. This appears to be due to diffraction of the reflected beam off wavelength-scale structure in the critical surface, a phenomenon reported by others, and generally present in such interactions [34]. This effect is actually obscured because the pulse undergoes strong phase modulation resulting in a greatly broadened spectrum of several hundred nanometers that blurs diffraction effects. We can reduce the blurring by spectrally filtering the reflected light. Figure 3.5(b) shows the output pulse spatial profile when only a $40 \mathrm{~nm}$ bandwidth centered about the $1 \mu \mathrm{m}$ carrier wavelength is measured for the $L=3 \mu \mathrm{m}$ case. Note that the fine structure is more pronounced and has a characteristic spatial scale that matches the spot size of the flat mirror reflected pulse 
(Figure 3.4, black lines). This is a signature of strong spatial phase variation being imposed on the pulse. This result from LsP constitutes the second major prediction of this work: Reflected light will show strong spatial variation if spectrally filtered, but will otherwise have a spot-like profile if the full spectrum is examined.

(a) $\mathrm{L}=0.3 \mu \mathrm{m}$

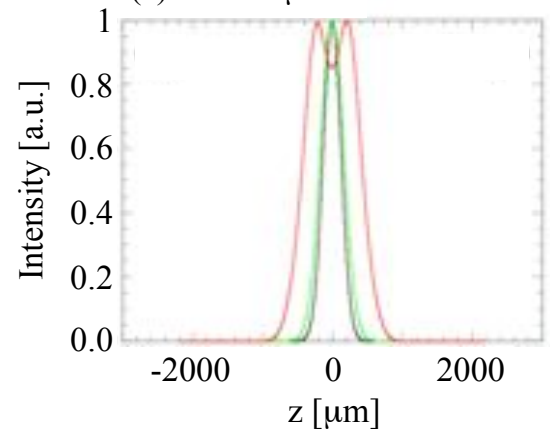

(b) $\mathrm{L}=1 \mu \mathrm{m}$

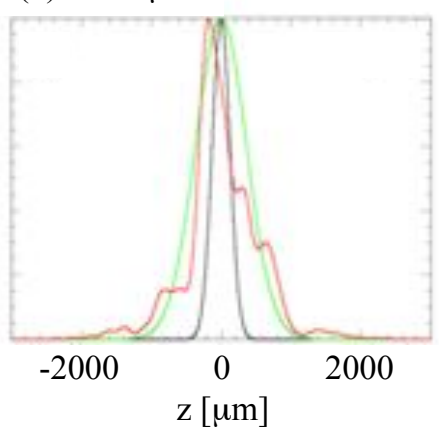

(c) $\mathrm{L}=3 \mu \mathrm{m}$

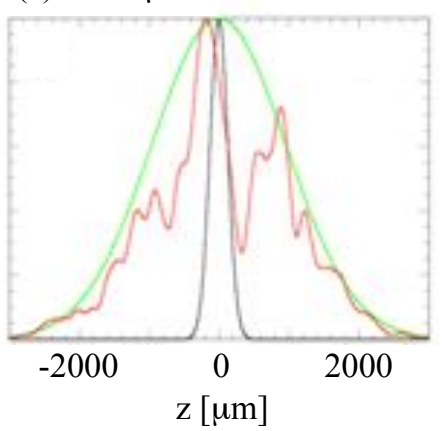

Figure 3.4: (Color) Pulse spatial profile $4 \mathrm{~mm}$ from the target. LsP results are compared to those of a simple model for pre-plasma scale lengths of (a) 0.3 , (b) 1.0, and (c) $3.0 \mu \mathrm{m}$. Black: profile for a perfect mirror reflection. For each case, the perfect mirror is the narrow line (black); the smooth Gaussian in each (green) is the model; the line with dips (red) is from LsP.

We note that the intensity is well above the critical threshold for self-focusing, which can also result in modification of the divergence of the reflected pulse. Apparently, given the agreement in overall divergence between our simple model and LSP, the effect of the shaped critical-surface dominates. We sought to isolate the contribution of self-focusing and performed an additional simulation with only pre-plasma present. The pre-plasma density increased up to the weak-field, $\gamma=1$, critical surface and then decreased back to vacuum. This allowed the pulse to suffer propagation effects with minimal reflection. Once back in vacuum, the pulse was propagated into the far field, as before. For a $3 \mu \mathrm{m}$ scale length (same as in Figure 3.4(c)), the propagation-only contribution to the beam divergence is shown in Figure 3.5(b). The beam divergence is increased but much less than that by the shaped critical surface. 

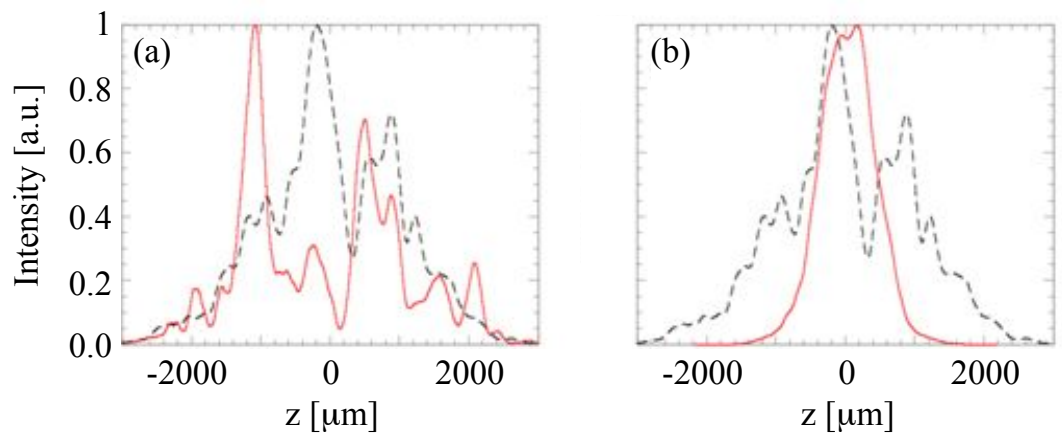

Figure 3.5: (Color) Far field, $L=3 \mu \mathrm{m}$ scale length result summary as described in the text: (a) spatial profile after spectral filtering using a $40 \mathrm{~nm}$ bandwidth filter and (b) spatial profile after propagation through pre-plasma only without reflection. The dotted line reproduces the LsP profile shown in Figure 3.4(c) for easy comparison.

\subsection{A new diagnostic and summary}

The results described here suggest that the reflected light of the main pulse itself provides an excellent, quantitative diagnostic for the amount of pre-plasma present in an experiment because the outgoing divergence is directly related to the pre-plasma density gradient. The omnipresent pre-plasma affects many aspects of experiment at relativistic intensities, yet tools for its measurement have been lacking. Our proposed diagnostic offers two advantages. First, it measures the pre-plasma density gradient at the relativistic critical surface, a region of primary interest that is opaque to most other techniques. Second, measurement of the divergence angle of the reflected light is not difficult or expensive, involving only a diffuser and camera. So long as the laser vacuum spatial profile and intensity are well characterized, the pre-plasma scale length can be determined by comparison to our simple model. If the initial laser spatial profile is far from Gaussian, a closed form expression for the output divergence may not be possible, but the predicted divergence can still be evaluated numerically. Finally, we note that this approach is primarily intended for situations where the scale length is expected to be $1 \mu m$ or larger. For very short scale length pre-plasmas, interpretation of the divergence becomes ambiguous. 
While we have not examined the case where the laser pulse breaks into a large number of filaments, such as occurs when larger intensities or longer pre-plasma scale lengths are present, we note that the pre-plasma can also be measured by using a second, lower intensity probe laser that does not suffer severe filamentation if this is an issue. The probe laser would need to be introduced after the pre-plasma has been established but before the primary laser arrives. This should usually be possible; however, the probe would also need to be offset spatially from the primary laser to avoid perturbing the experiment.

In summary, modification of the critical surface due to high intensity laser pulses has been noted for a long time [34]. We describe an overlooked effect of the shaped critical surface enhanced divergence of the reflected beam. We have developed a simple model that captures the primary effect. We propose this effect as a new pre-plasma diagnostic that is sensitive to the region near the critical surface that is currently difficult to characterize using current techniques.

We acknowledge useful discussion with D. Welch, M. Key, R. Stephens, and S. Wilks. This work was performed with support from DOE under Contract Nos. DE-FG0205ER54834 and DE-AC52-07NA27344 and a grant of computer time from the Ohio Supercomputer Center. 


\section{Chapter 4}

\section{DYNAMIC IONIZATION IN LOW-CONTRAST RELATIVISTIC LASER-PLASMA INTERACTIONS}

For the highly simplified study in the previous chapter, the charge state appeared to play little-to-no role in altering the far-field specular reflectivity measurements as compared to the effect of the shaped critical surface. This, however, is not the case for the experimentally more realistic pulses and pre-plasmas (Chapter 2.4, Figure 2.9) where dynamic ionization of the pre-plasma can dramatically alter not only the pulse, but also the interaction region. In this chapter, simulations with experimentally inspired low-contrast pulse conditions are studied using under both a fixed ionization state and one that is dynamically ionizing ${ }^{18}$ are compared in terms of the quantities of interest: specular reflectivity measurements and ensuing hot-electron generation.

Ultimately, the applications discussed in Chapter 1 are only interested in the hotelectrons that are generated in the ultra-intense interaction. Since these electrons are not directly observable near the interface in the experiment, $\mathrm{we}^{19}$ rely on simulations constrained by experimental observables of target and pulse properties to infer hot-electron properties. Specular diagnostics can provide invaluable (and perhaps most importantly, experimentally obtainable) insight into the laser-plasma interaction as the specularly reflected pulse. However, the phenomena that drive these specular modifications all occur near the interaction

\footnotetext{
${ }^{18}$ For this work, only sequential field ionization is considered.

${ }^{19} \mathrm{By}$ 'we', I refer to the relativistic laser-plasma interaction community as a whole. I exclusively performed the simulation work in this thesis, under the supervision and direction of D. W. Schumacher and A. Link.
} 
region, referred to here as the near-field. In order for these far-field measurements to be physically meaningful, they must be representative of the near-field interaction. Due to a variety of pulse-altering non-linear laser-plasma phenomena (discussed in Chapter 1.5.4), the interaction region can dynamically evolve in time and correspondingly affect the far-field properties of the specularly reflected pulse. Depending on the nature of the measurement, these temporal modifications can convolve the far-field answer to the point of misinterpreting the near-field physics of interest.

Using simulations, discussed in Chapter 4.1, I show how differences in ionization models result in temporal modifications in the electron density profile and laser-intensity distribution. Estimated near-field instantaneous wavelength shifts from ionization, relativistic self-phase modulation and motion of the critical surface and far-field reflectivity calculations are discussed in Chapter 4.2. Pulses are analytically propagated over millimeters to study far-field diffraction effects and several approaches are discussed in Chapter 4.2.2. Ensuing hot-electron generation from these evolving interaction regions is discussed in Chapter 4.3. For specular pulse properties characteristic of the experiment, I show how quasi-static magnetic field generation in the under-dense pre-plasma strongly influences supra-thermal electrons with energies above the quiver energy $(\sim 1 \mathrm{MeV})$. Finally, in Chapter 4.4, I summarize with a discussion of the implications for experimental measurements.

\subsection{PIC simulations}

\subsubsection{Setup}

$2 D 3 V$ Cartesian fully kinetic particle-in-cell simulations were performed to determine the influence of ionization dynamics in Aluminum targets affect specular reflectivity measurements and hot-electron generation in low-contrast laser-plasma interactions using the commercially available PIC code LsP [104]. The setup is shown in Figure 4.1. The initial target conditions (density, temperature and charge state) before the short-pulse arrived were taken from the Multi2D simulations (shown in Figure 2.9). Since the Multi2D calculation returns a cell averaged ionization state, I populate the LsP simulation using 
a linear combination of the surrounding charge states (i.e. between floor $(Z)$ and $\operatorname{ceil}(Z)$ ) with the same cell averaged charge state as MulTi2D. The pre-plasma was modeled out to $n_{c} / 100$, nearly $37 \mu m$ away from the original solid density interface and laser focal plane. The indentation of the target was also modeled and the solid density bulk had electron density $n_{e}=1.81 \times 10^{23} \mathrm{~cm}^{-3}$ with initially $Z=3$ charge state. The aluminum slab was $75 \mu \mathrm{m}$ deep by $150 \mu \mathrm{m}$ wide with $50 \mu \mathrm{m}$ of vacuum in front of the target with absorbing boundary conditions. Both incident and specularly reflected pulse field quantities were recorded at the inlet boundary of the simulation $(x=-50 \mu \mathrm{m})$. Hot-electron properties (time, energy and angle) are recorded as they pass through a diagnostic plane at $x=10 \mu \mathrm{m}$ inside solid density (approximately $5 \mu \mathrm{m}$ away from the new solid density interface) where $x=0 \mu m$ corresponds to the original solid density interface and incident laser focal plane (in vacuum).

LASER:

Polarized in the plane Normally incident

$\lambda_{\mathrm{L}}=527 \mathrm{~nm}$

$\tau_{\mathrm{FWHM}}=500 \mathrm{fs}, \sin ^{2}$

$\mathrm{Z}_{\mathrm{FWHM}}=8 \mu \mathrm{m}$ Gaussian (f/40)

$\mathrm{I}_{\text {peak }}=4.65 \times 10^{19} \mathrm{~W} / \mathrm{cm}^{2}$

SIMULATION:

LsP, direct-implicit

Absorbing boundaries

Electrons: 144-196 per cell

Ions: 25-49 per cell

Resolution: $\lambda_{\mathrm{L}} / 16, \mathrm{~T}_{\mathrm{L}} / 64$

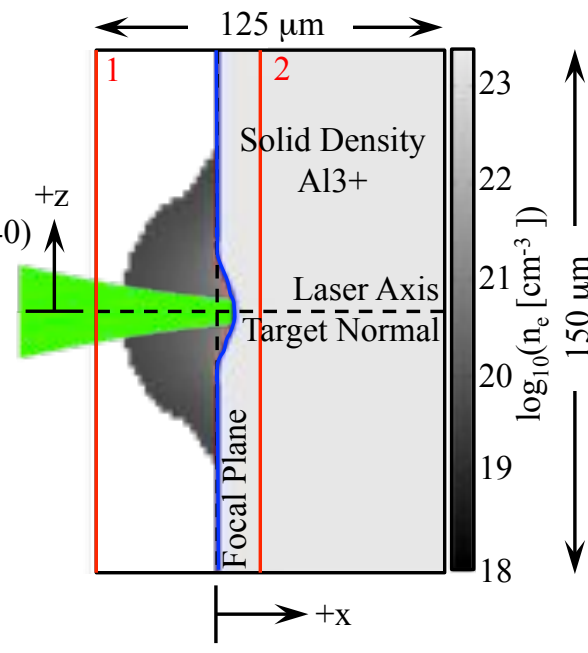

PLASMA TARGET:

$\uparrow$ Solid: initially $\mathrm{Al} 3+(5 \mathrm{eV})$

$\mathrm{n}_{\mathrm{e}}=1.81 \times 10^{21} \mathrm{~cm}^{-3}$

Multi2D pre-plasma: $n_{e}, T_{e / i}, Z$

Fixed or field ionization (ADK)

Fully kinetic electrons and ions

Collisionless

DIAGNOSTIC PLANES:

1. Laser fields $(\mathrm{x}=-50 \mu \mathrm{m})$

2. Hot-electrons $(\mathrm{x}=10 \mu \mathrm{m})$

Figure 4.1: (Color) Low-contrast specular reflectivity and absorption simulation setup. Laser (green) is incident normally on the MULTi2D pre-plasma (electron density shown on grayscale)

Modeled after the experiment, the incident laser pulse $\left(\lambda_{L}=527 \mathrm{~nm}\right.$, period $T_{L}=$ $1.76 \mathrm{fs}, n_{c}=4.0 \times 10^{21} \mathrm{~cm}^{-3}$ ) has a $1 \mathrm{ps}$ duration $\mathrm{sin}^{2}$ intensity profile with $500 \mathrm{fs}$ 
FWHM incident normally on the target with an $8 \mu \mathrm{m}$ focal spot $\mathrm{FWHM},{ }^{20}$ polarized in the simulation plane with a peak intensity of $4.65 \times 10^{19} \mathrm{~W} / \mathrm{cm}^{2}$. The peak quiver energy $U_{p}$ of electrons in the oscillating electric field is given by $U_{p}^{T W}=\left(\gamma_{o}^{T W}-1\right) m_{e} c^{2}=1.1 \mathrm{MeV}$ where $\gamma_{o}^{T W}=\sqrt{1+a_{o}^{2}}=3.2$ is the relativistic Lorentz factor and $a_{o}=e E_{o} / m_{e} \omega_{L} c=3.1$ is the normalized vector potential (where the ${ }^{T W}$ superscript refers to the vacuum value traveling-wave field structure).

The simulation was performed using a direct implicit algorithm incorporating an energyconserving particle push [104]. The LPI portion of the grid had a spatial resolution of $31.2 \mathrm{~nm}\left(\sim \lambda_{L} / 16\right)$ and temporal resolution of 27.5 as $\left(\sim T_{L} / 64\right)$. Through a separate study, specular pulse properties and hot-electron distributions in time, space, energy and angle were found to be sufficiently converged at these resolutions. Electron macro-particle densities ranged from 144 to 196 per cell and the ions ranged from 25 to 49 per cell resulting in $\sim 500-750 M$ macro-particles in the simulation. All electron and ion species were fully kinetic and collisionless.

Understanding how interfaces evolve is critical to understanding how electrons are generated. For low $\mathrm{Z}$ targets, this effect may be minimal but interactions with mid to high Z materials may increase local electron density by an order of magnitude (see Figure 1.6). The extent to which these interactions with Aluminum are sensitive to ionization dynamics is a priori unclear because of the non-uniformity of the charge state distribution. To study effect of dynamic ionization, two separate simulations are compared with (1) fixed and (2) sequential tunneling field ionization (using ADK (Ammosov, Delone and Krainov) ionization rates for ions in an alternating electromagnetic field [87]).

\subsubsection{Interaction region evolution}

The evolution of the electron density (given by $n_{e} / n_{c}$ ), charge state $Z$ and laser intensity $I$ for the fixed and dynamic ionization cases are shown in Figuress 4.2 and 4.3 respectively. The different rows show different shap-shots in time of these three quantities (separated

\footnotetext{
${ }^{20}$ The spatial profile was approximately Gaussian with an $f / 40, e^{-2}$ full angle divergence. This is drastically different from he experimental conditions, closer to an $f / 2.5$. To reproduce such a divergence in LsP, one would likely have to perturb the wave-fronts but this is beyond the scope of this work.
} 
by columns): the first row is the initial conditions, second, third and fourth are the rising half-peak, peak and falling-half peak intensity upon reflection respectively. In each subplot, the initial critical surface is indicated by the gray curve and the current relativistic critical surface with the black.

At first glance, these two simulations appear to evolve quite similarly, but a closer inspection reveals some subtle, yet interesting, differences. For the fixed ionization case, the critical surface at all times has been pushed toward solid density. The dynamically ionized case, on the other hand, shows the increased electron density at early times which initially pushes the critical surface away from the solid density interface but this expanding motion is eventually overcome by the peak of the pulse. By the rising half-peak of the pulse, the charge state for the dynamically ionizing case has saturated at $A l 11+$ in the laser interaction region. Leading up to the rising half-peak, the laser in each case has broken up into approximately 4 or 5 filaments. By the peak of the pulse, the filaments have nearly coalesced down into nearly a single filament for the fixed ionization run while the dynamic ionization case saw remained spread out over approximately two main filaments. This differences are likely due to the effects of ionization induced defocusing (discussed in Chapter 1.5.4) counteracting the laser self-focusing and, correspondingly, we see that the perturbations in the relativistic critical surface are significantly smoother when ionization is present. 

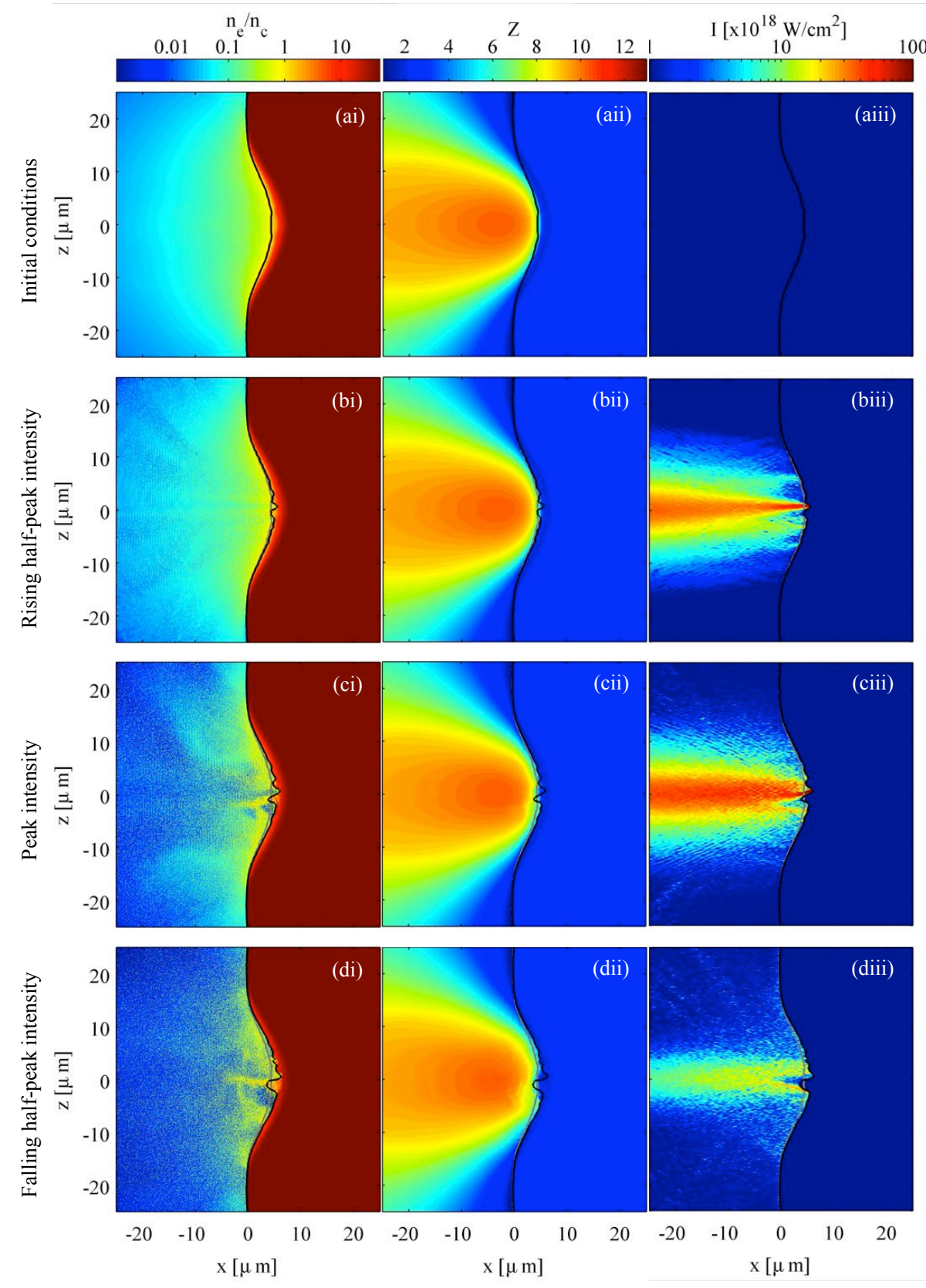

Figure 4.2: (Color) Simulated evolution of the low-contrast laser-matter interaction using fixed ion charge state at various snapshots in time, indicated by the different rows: (a) initial conditions, (b) rising half-peak upon reflection, (c) peak upon reflection and (d) trailing half-peak upon reflection. The first column (indicated by $i$ ) is the electron density profile, normalized to critical density, shown on a $\log _{10}$ scale. The second column (indicated by $i i$ ) is the cell averaged charge state. The third column (indicated by $i i i$ ) is the near-field cycle averaged laser intensity also shown on a $\log _{10}$ scale. The initial and current relativistic critical surfaces are indicated by the gray and black curves respectively. 

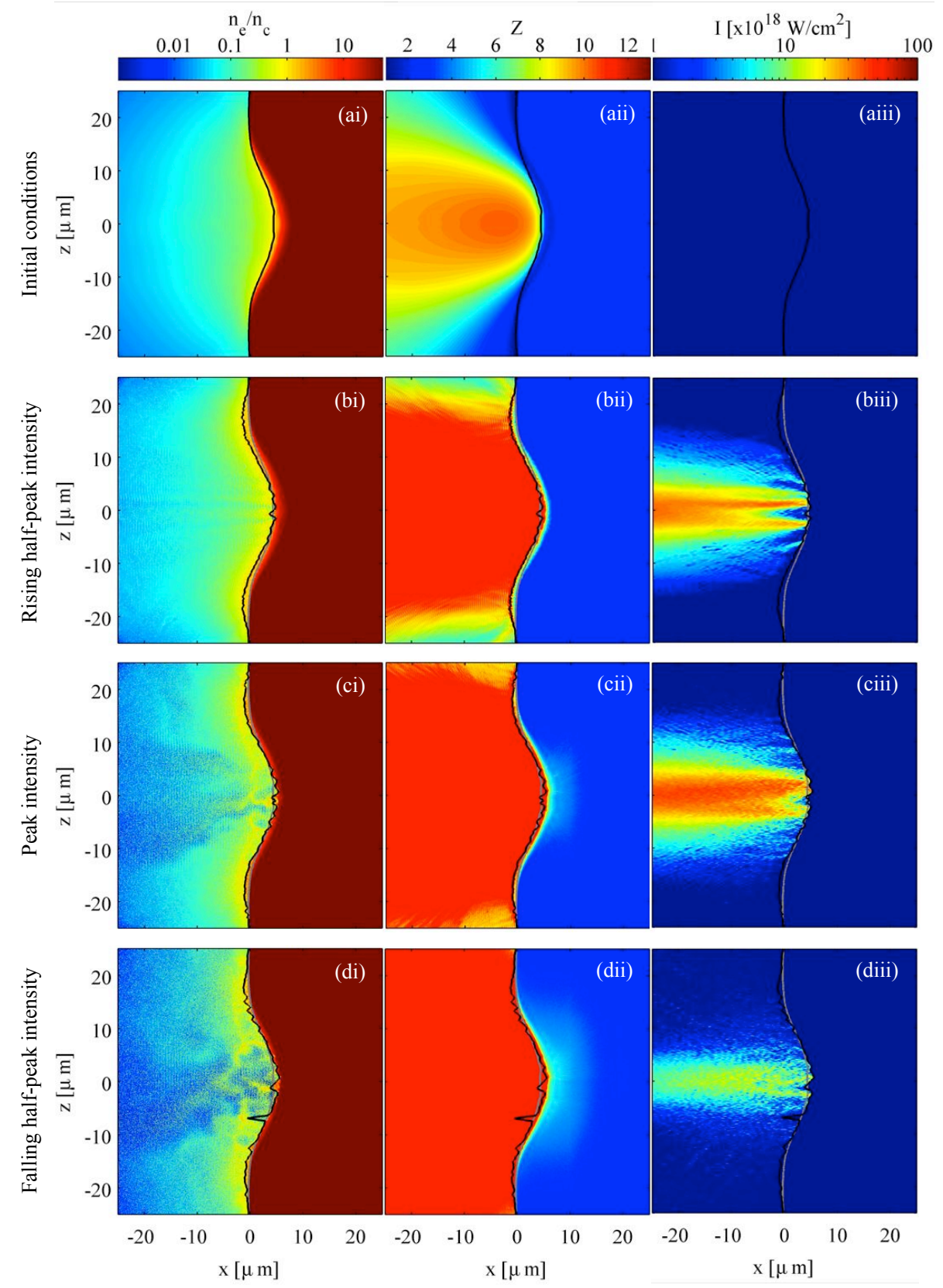

Figure 4.3: (Color) Simulated evolution of the low-contrast laser-matter interaction using dynamic field ionization at various snapshots in time, indicated by the different rows: (a) initial conditions, (b) rising half-peak upon reflection, (c) peak upon reflection and (d) trailing half-peak upon reflection. The first column (indicated by $i$ ) is the electron density profile, normalized to critical density, shown on a $\log _{10}$ scale. The second column (indicated by $i i$ ) is the cell averaged charge state. The third column (indicated by $i i i$ ) is the near-field cycle averaged laser intensity also shown on a $\log _{10}$ scale. The initial and current relativistic critical surfaces are indicated by the gray and black curves respectively. 


\subsection{Near-field physics and far-field specular reflectivity mea- surements}

Although subtle from electron density plots, the differences between the fixed and dynamically ionizing plasmas become abundantly clear in the near-field properties of the specularly reflected pulse. Characterization of the specularly reflected pulse near the interaction with simulations, however, is complicated by the presence of the supra-critical plasma target by electrostatic fields, quasi-static magnetic fields, noise from the kinetic nature of the macro-particles and, of course, the interference between the incident and reflected pulses. Instead, specular field properties are recorded at the inlet/outlet boundary of the simulation (50 $\mu \mathrm{m}$ away from the target interface) where these influences are negligible. However, even a few tens of microns away, diffraction effects have drastically reshaped the specular pulse from the desired measurement near the interaction interface. Instead, estimates of the pulse-altering phenomena discussed in Chapter 1.5.4 are made to determine their relative influences on the interaction in the near-field, discussed in Chapter 4.2.1. Propagating the light to the far-field within these same simulations, where the experimental measurements would be made, is prohibitively expensive since we would have to resolve the light propagation over millimeters or even centimeters of space before the diffraction pattern would converge. Instead, far-field properties of the specularly reflected pulse are obtained by analytically propagating the boundary measurements to the far-field, centimeters away. Multiple techniques exist and are discussed in Chapter 4.2.2. Far-field pulse properties are discussed in Chapter 4.2.3.

\subsubsection{Near-field physics}

All the interesting physics that affects the specular pulse properties, such as absorption, relativistic non-linearities, hole-boring, field ionization, et cetera, occurs near the interaction interface and many of these phenomena are nonlinearly dependent on intensity and electron density profiles. Since the pulse has a finite focal spot (Figure 2.8), we can expect there to be some spatial dependence on these effects in the near-field. 
Previous experimental work [35], which partially inspired the work in this thesis, has shown that the instantaneous spectral shifts for a low-contrast relativistic laser-plasma interaction arose primarily from the motion of the relativistic critical surface, ${ }^{21}$, i.e. a Doppler shift resulting in 'red' light for receding surfaces and 'blue' light for expanding surfaces. The rising edge of the pulse saw redshifts as large as $\approx 6 \%$, indicating a recession velocity of $\approx 3 \%$ of the speed of light, which decreased to near zero shift by the peak of the pulse, eventually turning into a blue shift. This dynamic shifting of the instantaneous wavelength arise from the dynamic competition between laser profile steepening and heated plasma expansion, where on the rising edge the increasing laser intensity pushed the critical surface towards solid density trailing edge of the pulse the plasma expansion overcame the decreasing laser intensity.

Shown in Figure 4.4 are spatially and temporally resolved estimates of the near-field spectral shifts (given as a percentage of the incident pulse carrier) due to (i) Doppler shifting from motion of the relativistic critical surface and (ii) relativistic self-phase modulation and (iii) their combined effect for (a) the fixed and (b) the dynamically ionizing simulations. The Doppler shift was calculated by tracking the motion of the relativistic critical surface (with a $20 \mathrm{fs}$ resolution, indicated by the black curves in Figures 4.2 and 4.3). The Doppler shift upon reflection is then simply given by Equation 1.32. The relativistic self-phase modulation was estimated, following Watts [42], using Equation 1.30 and the temporally evolving under-dense plasma density from the simulation. ${ }^{22}$ In each case, the incident laser intensity profile is assumed to be unperturbed. For clarity, the 1 and $50 \%$ peak intensity contours of the laser are indicated by the dashed black curves and any intensity below $0.1 \%$ is truncated (shaded gray areas).

For the fixed ionization case (a), the estimation of the Doppler shift from the motion

\footnotetext{
${ }^{21}$ No ionization/recombination effects were considered (as the pre-plasma was almost fully ionized according to the rad-hydro simulations) and relativistic self-phase modulation was estimated to be approximately 3 times smaller than the observed shifts, around $2 \%$. Other phenomena, such as the changing location of the relativistic critical surface and initial motion of the pre-plasma before the main pulse arrived was found to be negligible.

${ }^{22}$ In particular, the integral was performed up to the relativistic critical surface and no phase modulation of the backwards propagating pulse was included (which should be significantly less since the specularly reflected pulse has significantly lower intensity).
} 

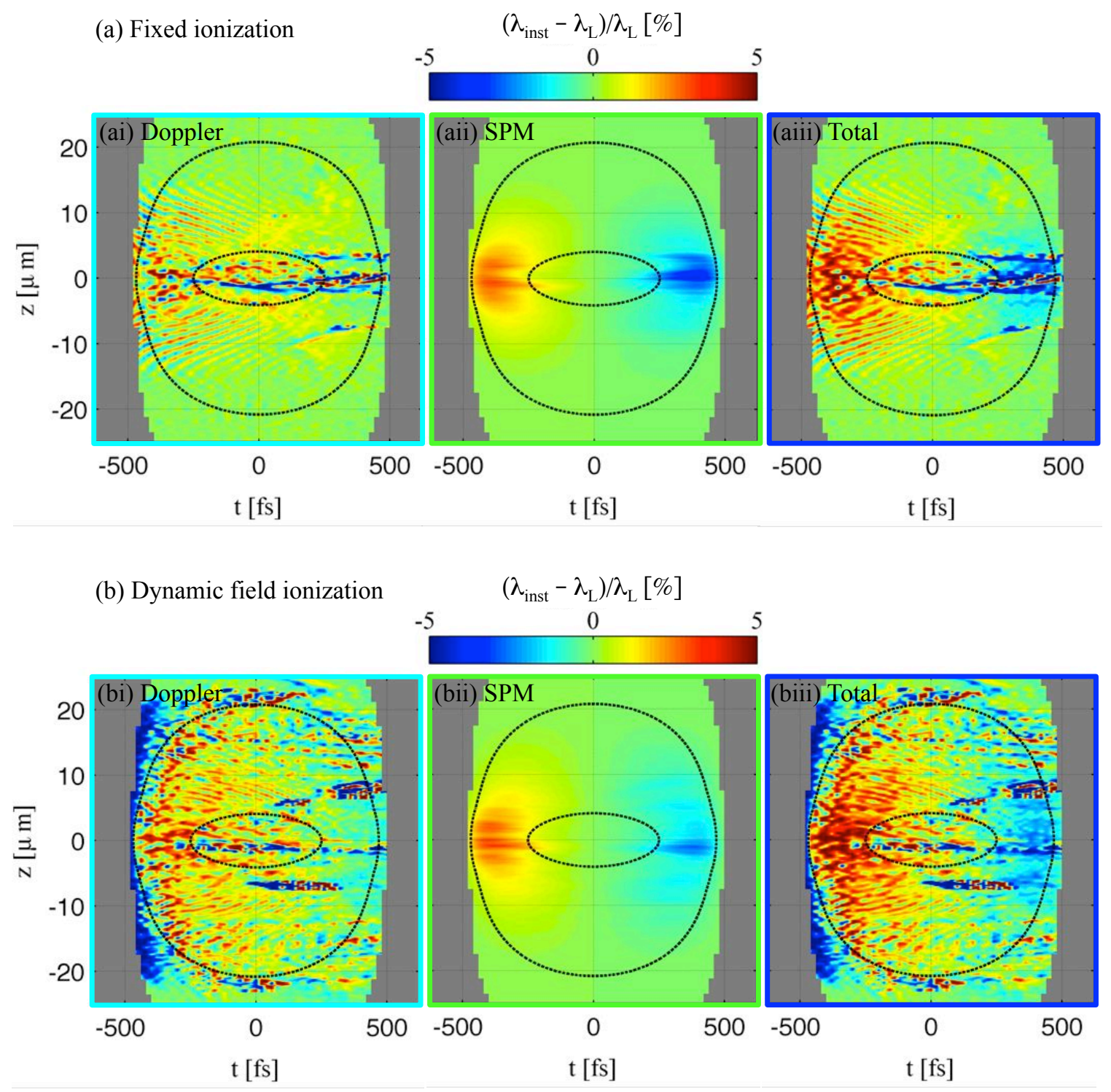

Figure 4.4: (Color) Near-field estimates of spectral shifting of the specularly reflected pulse for (a) fixed and (b) dynamic field ionization cases. The spatial striations are due to laser filamentation and electron density modification.

of the relativistic critical surface (ai) suggests an early redshift $(\sim+1.5 \%)$ that decreases in time to near zero. Spatial 'fringes' in the distribution on the rising edge of the pulse, due to the rippling of the critical surface from the laser filamentation, coalesce down to only a few by the trailing edge of the pulse, consistent with our previous assessment. The 
relativistic self-phase modulation (aii) suggests shifting of $\sim \pm 2 \%$. The combined influences of relativistic self-phase modulation from propagation through the plasma and Doppler shifting from reflecting off the moving relativistic critical surface is shown in (aiii), given by the relation

$$
\frac{\Delta \lambda_{\text {total }}}{\lambda_{L}}=\frac{\Delta \lambda_{\text {Doppler }}}{\lambda_{L}}+\frac{\Delta \lambda_{S P M}}{\lambda_{L}}+\frac{\Delta \lambda_{\text {Doppler }}}{\lambda_{L}} \frac{\Delta \lambda_{S P M}}{\lambda_{L}}
$$

where $\Delta \lambda=\lambda_{\text {inst }}-\lambda_{L}$.

The field ionization in (b) raised the local electron density and drove a Doppler blueshift (up to $\sim-5 \%$, although short-lived) just from the motion of the critical surface away from solid density (which was absent in (ai)). Only lasting $\approx 50 \mathrm{fs}$, this is consistent with the rise time for the laser to reach $\sim 10^{18} \mathrm{~W} / \mathrm{cm}^{2}$, an intensity sufficient to strip the Aluminum ions to the highest charge state achievable with this laser $(A l 11+)$. The expansion of critical surface is then quickly overcome by the increasing laser intensity, turning into an even stronger redshift $(\sim+2.5 \%)$ than what was observed for the fixed ionization case. Once again, this redshift gradually disappeared by the peak of the pulse, turning into a slight blueshift on the trailing edge. Although still present, the spatial striations in the profile are less defined than the fixed charge state simulation, consistent with the previous assessment that ionization defocusing counteracted the laser filamentation resulting in a smoother relativistic critical surface. The instantaneous wavelength shifts due to SPM was estimated to be around $+2 \%$ on the rising edge and $-1 \%$ on the trailing. If the electron density profile were consistent throughout the simulation, the SPM should have created equal strength red and blueshift. This suggests that the under-dense electron density leading up to the relativistic critical surface is significantly different on the rising edge of the pulse compared to the trailing edge (as compared to the fixed ionization case in (a)).

\subsubsection{Analytic pulse propagation techniques}

Although being computationally intractable or prohibitively costly in the PIC simulations, producing far-field pulse phenomenon centimeters away is analytically approachable. Two techniques were addressed: (1) paraxial propagation using the Fresnel propagation kernel 
implemented in Chapter 3 and (2) a novel non-paraxial propagation approach simply using the wave-equation. Both solutions start with the wave equation and the ansatz of a linearly polarized plane wave (parallel with the $z$-axis) with wavelength $\lambda_{L}=2 \pi / k_{L}$, angular frequency $\omega_{L}=c k_{L}$, spatial envelope $u$ and temporal envelope $\epsilon$ propagating along the $x$-axis (Equation 4.2), using the geometry of the simulation setup in Figure 4.1.

$$
\begin{aligned}
\left(\frac{\partial^{2}}{\partial x^{2}}+\frac{\partial^{2}}{\partial y^{2}}+\frac{\partial^{2}}{\partial z^{2}}-\frac{1}{c^{2}} \frac{\partial^{2}}{\partial t^{2}}\right) \vec{E}(x, y, z, t) & =0 \\
\vec{E}(x, y, z, t) & =u(x, y, z) \epsilon(t) e^{i\left(\omega_{L} t-k_{L} x\right)} \hat{z}
\end{aligned}
$$

After a separation of space and time variables and a Fourier transform along the spatial dimensions transverse to beam propagation direction (i.e. along $y$ and $z$ ), this evolution of the spatial envelope as a function of propagation distance $x$ takes the form of Equation 4.4 where $\widetilde{u}$ is the Fourier transform of the field along the transverse spatial dimensions.

$$
\begin{gathered}
\left(\frac{\partial^{2}}{\partial x^{2}}-2 i k_{L} \frac{\partial}{\partial x}-\left(k_{y}^{2}+k_{z}^{2}\right)\right) \widetilde{u}\left(x, k_{y}, k_{z}\right)=0 \\
\widetilde{u}\left(\overrightarrow{k_{\perp}}, k_{L}, \Delta x\right)=\widetilde{u}\left(\overrightarrow{k_{\perp}}, k_{L}, x_{o}\right) e^{i \frac{k_{\perp}^{2}}{2 k_{L}} \Delta x}
\end{gathered}
$$

If we assume a slowly varying transverse spatial envelope $\left(i . e . k_{\perp}^{2}=k_{y}^{2}+k_{z}^{2}<<k_{L}^{2}\right.$, where $\left.\overrightarrow{k_{\perp}}=\left(k_{y}, k_{z}\right)\right)$, then Equation 4.4 reduces down to the elegant form of the Fresnel propagator given by Equation 4.5 [141]. Simply put, the far-field spatial field distribution a distance $\Delta x$ away from the measurement plane at $x_{o}$ is obtained through an appropriate phase addition in k-space of the near-field answer. For small Fresnel numbers $\left(F=a^{2} /\left(\Delta x \lambda_{L}\right) \ll 1\right.$ where $a$ is the characteristic size of the aperture), this approach has been found to reliably reproduce the far-field Fraunhofer diffraction patterns produced by single and double slits, round and square apertures as well as the endearing Gaussian beam solution.

Since there is some finite bandwidth in short pulses, this propagator kernel should be applied to each frequency individually. ${ }^{23}$ Starting with the space/time domain of the field quantities at boundary of the simulation, a Fourier transform along both dimensions results

\footnotetext{
${ }^{23}$ Note, this technique hadn't been adopted for use in the previous chapter yet.
} 
in a wave-number/angular frequency $(k-\omega)$ domain of the pulse. The Fresnel propagator kernel can then be applied at each and every $k-\omega$ pair before being inverse-Fourier transformed to re-obtain the space/time domain of the pulse after propagation. This is equivalent to propagating each laser frequency individually, treating each wave-number/angular frequency component as a plane-wave, then superimposing the solutions after propagation.

However, rippling of the critical surface in low-contrast interactions can also create features smaller than the laser wavelength. In order to appropriately handle these larger $k$ modes, we approached the propagation problem by first removing the paraxial assumption which allowed for the propagation of light modes up to $k_{L}{ }^{24}$ The catch, however, in dropping the paraxial approximation is that the differential equation to solve becomes second order, requiring two boundary conditions to solve. Although not as elegant as the Fresnel propagator kernel, one solution of the non-paraxial wave equation takes the form of Equations 4.6-4.9 for the case of having field measurements at two planes located at $x_{1}$ and $x_{2}{ }^{25}$ where

$$
\begin{aligned}
\widetilde{u}\left(\overrightarrow{k_{\perp}}, x\right) & =\mathcal{C}\left[\alpha(x) \widetilde{u}\left(\overrightarrow{k_{\perp}}, x_{1}\right)+\beta(x) \widetilde{u}\left(\overrightarrow{k_{\perp}}, x_{2}\right)\right] \\
\mathcal{C} & =-\frac{e^{-i k_{L}\left(x_{1}+x_{2}\right)}}{2}\left(1-i \cot \left[\left(x_{1}-x_{2}\right) \sqrt{k_{L}^{2}-k_{\perp}^{2}}\right]\right) \\
\alpha(x) & =e^{i k_{L}\left(x+x_{2}\right)+i\left(x_{1}-x\right) \sqrt{k_{L}^{2}-k_{\perp}^{2}}}\left(1-e^{2 i\left(x-x_{2}\right) \sqrt{k_{L}^{2}-k_{\perp}^{2}}}\right) \\
\beta(x) & =e^{i k_{L}\left(x+x_{1}\right)+i\left(x-x_{2}\right) \sqrt{k_{L}^{2}-k_{\perp}^{2}}}\left(1-e^{2 i\left(x+x_{1}\right) \sqrt{k_{L}^{2}-k_{\perp}^{2}}}\right)
\end{aligned}
$$

with constant $\mathcal{C}$, dependent only on the boundary conditions, $\widetilde{u}\left(\vec{k}_{\perp}, x_{1}\right)$ and $\widetilde{u}\left(\vec{k}_{\perp}, x_{2}\right)$ are the $k$-space fields at $x_{1}$ and $x_{2}$ respectively, and the $x$ dependence comes in through $\alpha$ and $\beta$. Alternative solutions exist for different boundary conditions but the two plane approach was the most practical for these simulations. ${ }^{26}$ The procedure for propagating the pulse is identical to the paraxial case, again modifying the phase of the near-field solutions in $2 D$

\footnotetext{
${ }^{24}$ It is possible that this analysis could be extended to beyond $k_{L}$, but this has been neglected for now since there is very little energy in these modes and the simulations do not properly resolve features significantly smaller than the laser wavelength.

${ }^{25}$ For previous simulations in Chapter 3 enough vacuum was placed in front of the target to obtain a spatially resolved field measurement, away from under-dense plasma, as compared to the novel non-paraxial approach which uses a diagnostic plane, resolved in space and time.

${ }^{26}$ In practice, this approach has worked the best for two planes separated by only a few simulation grid points.
} 
Fourier space and then returning to the space-time domain with inverse Fourier-transforms.

To test the validity of these algorithms, simulations without any pre-plasma were performed using roughened target surfaces with both laser spot size and sub-wavelength scale perturbations. $^{27}$ Shown in Figure 4.5 are the power spectra of both the target surface roughness (green) and of the specularly reflected pulse as measured at the boundary of the simulation $($ black $) .^{28}$ The spectrum of the rough target has clearly been mapped to specularly reflected pulse and into spectral modes up to and beyond sub-wavelength features $\left(k_{L} \geq 2 \pi / \lambda_{L}=11.9 \mathrm{rad} / \mu \mathrm{m}\right.$, indicated by the vertical dashed red line). Also shown is the approximate valid range of the paraxial assumption ( red), which only covers approximately an order of magnitude in spectral intensity. The specularly reflected pulse is inherently nonparaxial; depending on the relative contribution of these modes to the spectra, propagating these modes paraxially or simply ignoring them could potentially introduce significant error. The region of the spectra valid in this non-paraxial approach is also shown (blue) and covers an additional order of magnitude in intensity.

The validity of both the paraxial (Equation 4.5) and non-paraxial (Equation 4.6) propagation techniques was tested by propagating the pulse both forwards to the far-field and backwards toward the original laser focal plane. By propagating the field measured at the boundary of the simulation backwards toward the original laser focal plane, we can directly see how the surface perturbations are imprinted onto the spatial profile of the specularly reflected pulse. Shown in Figure 4.6 are the propagated pseudo-intensity distributions of the specularly reflected pulse (i.e. $\left|E_{z}\right|^{2}$ ) as a function of space and time at the original laser focal plane (on a $\log _{10}$ color scale so that the shape of the phase-fronts are apparent). These images are zoomed in around small window that is centered in space and time around the peak laser intensity. ${ }^{29}$ The paraxial approach (filtered in $k$-space to only a valid region where $k_{T}^{2} \leq k_{L}^{2} / 10$ ) was used in (a) and the non-paraxial approach for (b). Also shown is

\footnotetext{
${ }^{27}$ The presence of under-dense plasma is not incorporated into this model so the propagation is only valid in vacuum

${ }^{28}$ In fact, these features are characteristic of the experimental conditions, discussed in more detail in Chapter 6.

${ }^{29}$ This test was performed using the shorter $100 \mathrm{fs}$ pulses where the original surface perturbations have not been altered by the peak of the pulse.
} 


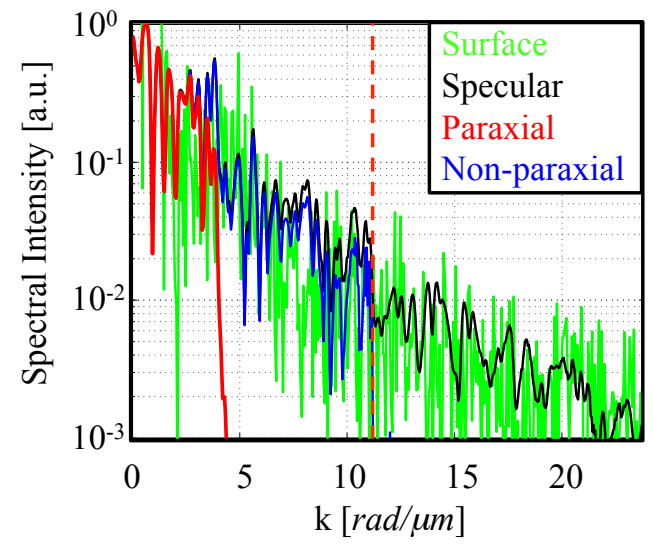

Figure 4.5: (Color) Spectral intensity distributions of the surface (green) and the specularly reflected pulse (black). Portions of the spectrum valid for paraxial propagation (red) and non-paraxial approach (blue) are also shown where the laser wave-number $k_{L}$ indicated by the vertical dashed red line.

the original realistic surface roughness time of flight (i.e. $2 \delta x / c$ where $\delta x$ is the deviation of the surface from the laser focal plane) in red. Clearly, the filtered paraxial is incapable of capturing the small scale features of interest (again, not actually what it was intended to be used for) whereas the non-paraxial does in quite good detail (also validating the technique). Propagation to and from the far-field of this non-paraxial technique was also tested using a paraxial gaussian pulse, single slit, and double slit, all of which were found to be reliably reproduced.

\subsubsection{Far-field specular reflectivity}

Using the non-paraxial propagation approach previously discussed, the far-field properties of the specularly reflected pulse can now be studied and compared (at least qualitatively) to the near-field physics estimates. Quantities of particular interest, as inspired by the experiment, are the temporally integrated spatial intensity distribution, spatially integrated spectrum, and spatial uniformity of the temporally resolved instantaneous wavelength shifts. Shown in Figure 4.7 are these integrated properties of the far-field specular pulse, $10 \mathrm{~mm}$ 
(a) Paraxial

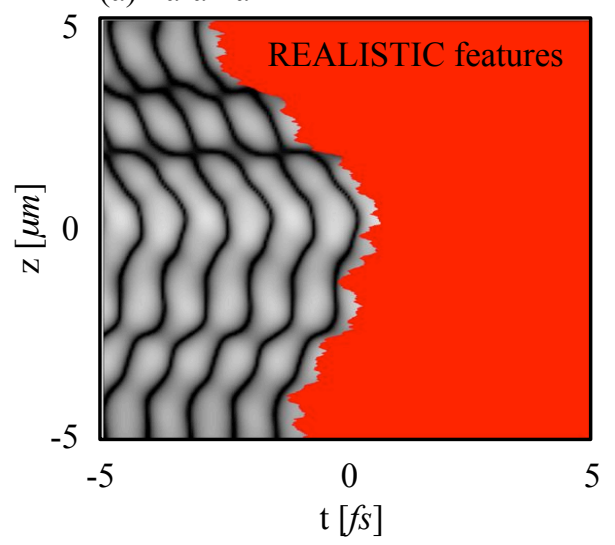

(b) Non-paraxial

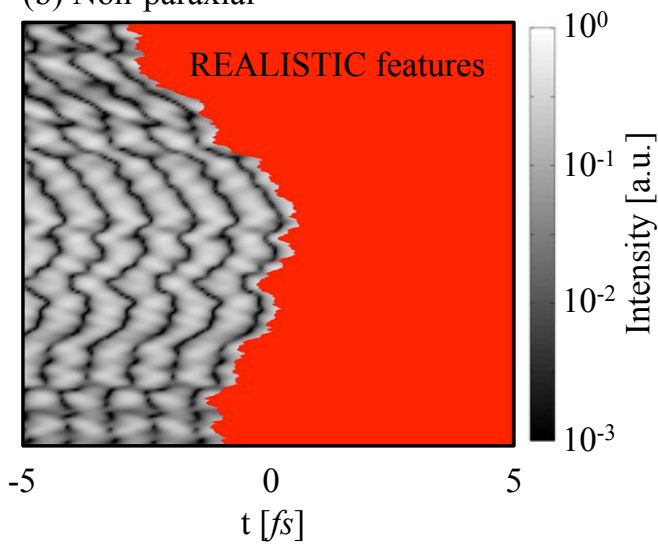

Figure 4.6: (Color) Phase-front distortions in the specularly reflected pulse at the $x=$ $0 \mu m$ focal plane, realistic surface roughness shown in red, using the (a) filtered paraxial propagation approach and (b) non-paraxial approaches.

away from the focal plane, for (first column) the fixed ionization simulation and (second column) the dynamically ionizing simulation. The top row contains the temporally integrated spatial intensity distribution, normalized to the peak, with the experimental Spectralon ${ }^{\circledR}$ acceptance window shown by the vertical black lines. The most obvious difference between the two runs is in the 'wings' of the distribution where the fixed ionization case (a) has local peaks near the edge of the pulse and the field ionization case (b) is significantly smoother. These are significantly much more divergent pulses than in the experiment, likely due to the fact that the simulation used an $\sim f / 40$ Gaussian compared to the experimental $f / 3$ flattop. Just from these spatial distributions, it becomes apparent how sensitive the far-field measurements are to the near-field physics. The reflectivity $R$ (defined to be the fraction of the incident pulse energy that reflected into $2 \pi$ steradians within a $75 \mathrm{~nm}$ bandwidth about the $527 \mathrm{~nm}$ carrier) of the simulation is also lower for the field ionization case $(20.8 \%$ and $14.3 \%$ for the fixed and field ionization cases respectively), suggesting that the absorption into hot-electrons could correspondingly be higher. If this pulse were measured in the experiment, only $\approx 40 \%$ of the beam energy in either case would have been captured (clearly, these are more strongly diverging pulses than in the experiment). This fraction 
of the reflected pulse energy, referred to here as specular reflectivity $R_{S}$, would have only amounted to around $7 \%$ reflectivity (the experiment suggested it should be closer to $15 \%$ ).

The second row shows the integrated spectra for both the incident (black) and specular (red) pulses. For either case, the specular pulse is $\gtrsim 10 \times$ broader, approximately $9.6 \mathrm{~nm}$ and $12.4 \mathrm{~nm}$ compared to the incident $1.3 \mathrm{~nm}$ FWHM. Both specular spectra are also strongly redshifted to $\approx 535 \mathrm{~nm}$, an overall $1.5 \%$ redshift. (As a reminder, the experimental data suggested an overall blueshift of $1 \mathrm{~nm}$, centered at $526 \mathrm{~nm}$ with only $\sim 2.5 \times$ broadening.)

The spatially integrated, intensity weighted instantaneous spectral shifts (red) are shown in the third row, along with the instantaneous intensity (black). Just as suspected from the evolution of the electron density in the simulations, the ionization on the leading edge of the pulse has created a noticeable difference in the instantaneous spectral shifts. For the fixed ionization case in (e), the pulse redshifts to around $2 \%$ before decreasing and turning into a $-1 \%$ blueshift on the trailing edge of the pulse. The field ionization in (f) however creates an early blueshift of $\approx-3 \%$, before being overcome by the rising intensity of the laser pulse and turning into an even larger $\approx 3 \%$ redshift, which again falls off gradually after the peak of the pulse reflects, turning into $\sim-1 \%$ blueshift. Unlike the experiment, very little temporal broadening of the pulse occurs in either simulation but both appear to be slightly temporally steepened (Chapter 1.5.4). Assuming that the density profile is constant and the laser has the incident laser temporal profile, profile steepening of the peak is estimated to be only about $-3.7 f s$, negligible compared to the $\sim 100 \mathrm{fs}$ observed. ${ }^{30}$ The temporal profile modification of the specular pulse is likely due to absorption and possibly self-focusing effects and almost certainly not group velocity dispersion.

Shown in Figure 4.8 is the far field $(x=-10 \mathrm{~mm})$ spatial and temporally resolved (left column) intensity and (right column) instantaneous wavelength shifting of the specularly reflected pulses for both the (top row) fixed and (bottom row) dynamically ionization pre-

\footnotetext{
${ }^{30} \mathrm{SPM}$ can, however, broaden the spectrum, resulting in greater GVD and creating a stronger effect. Including the estimated SPM shifts, the peak of the pulse would only have sharpened by $-4 f s$ relative to the rising red edge of the pulse, again negligible compared to the pulse duration.
} 
Fixed ionization
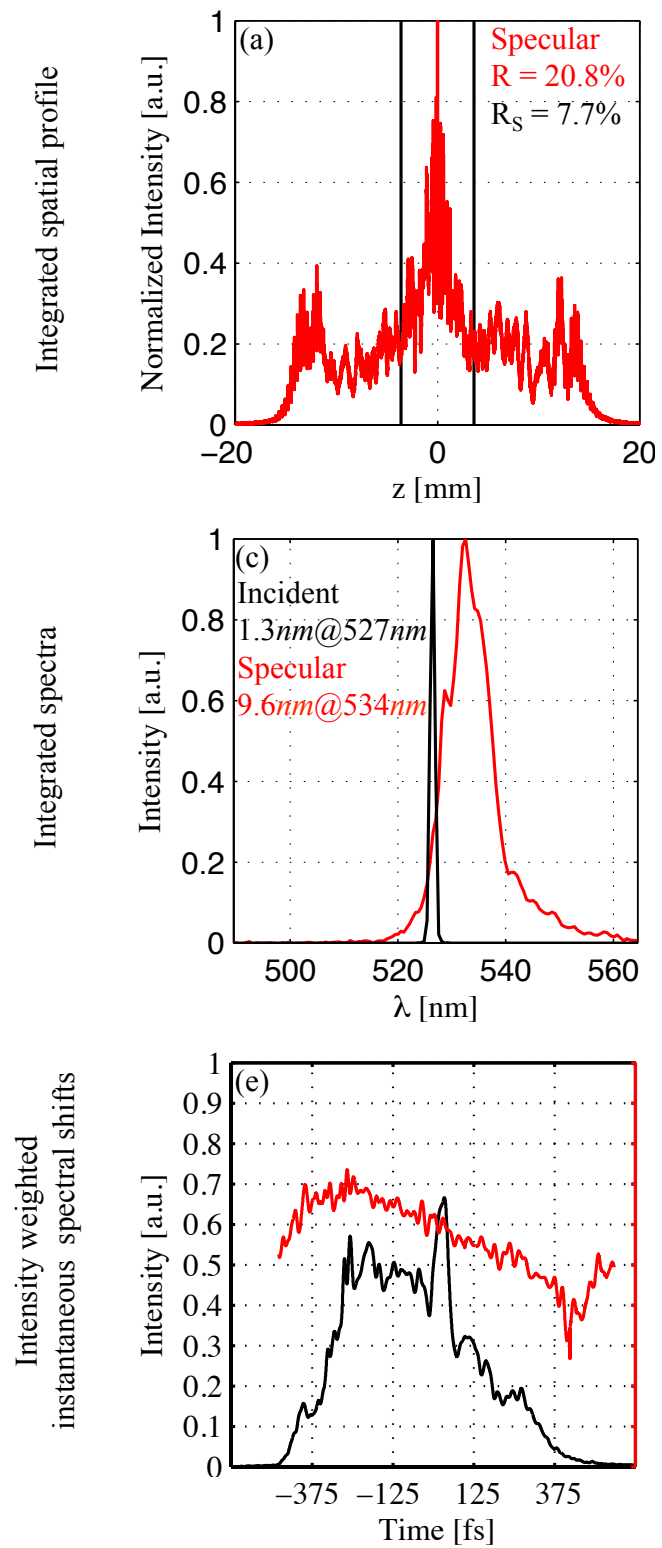

Dynamic field ionization
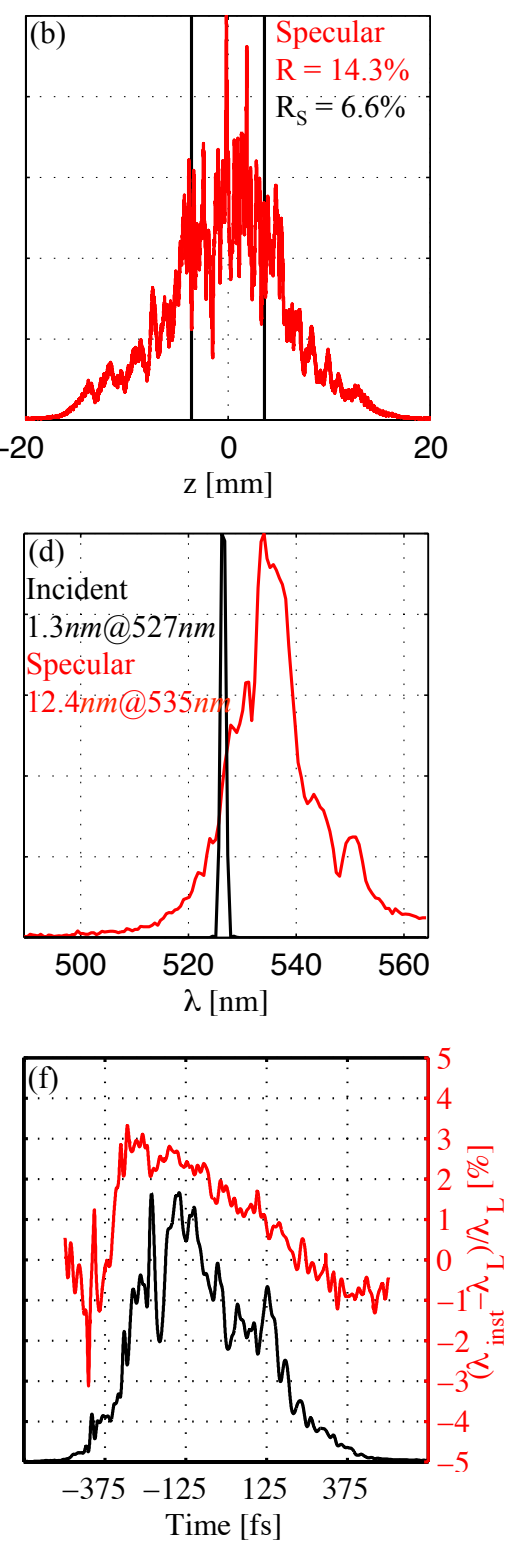

Figure 4.7: (Color) Integrated distributions of the specularly reflected pulse for (first column) fixed and (second column) field ionization simulations in the far-field $10 \mathrm{~mm}$ away from focus. Temporally and spectrally integrated spatial profiles (first row), spatially and temporally integrated spectra (second row) and spatially integrated instantaneous intensity and wavelength (third row). 
plasma. ${ }^{31}$ The intensity distribution for the fixed ionization simulation in subplot (a) shows that the 'wings' in the spatial intensity distribution develop near the peak of the pulse. Surprisingly, we find that the strongest redshifts in the far-field occur off the specular axis. The redshifts in the near-field are attributed to Doppler shifts from the electron density profile steepening, which is strongest for the highest intensities. The critical surface in these high intensity regions, because of the filamentation instability, is quite rippled as a result which drives stronger diffraction of the specularly reflected pulse and is likely the cause of the redshift appearing off axis. This suggests that any experimental measurement that is not spatially integrated, like the FROG, could be sensitive to finite spatial sampling. For the dynamically ionizing simulation, we find more uniform intensity and spectral shifts in space which is likely due to the less dynamic, smoother shape of the reflecting surface.

Since experimentally obtained FROG traces are nonlinearly sensitive to the intensity of the pulse (see Appendix C for more details on FROG measurements), appropriate weighting of simulation results must be applied before any comparison can be made between the two. Shown in Figure 4.9 are the spatially and temporally resolved answers, repeated from Figure 4.8 for convenience. In the right column are the spatially integrated, intensity weighted answers from the near-field estimates and the far-field calculations. Since, the PG FROG signal is proportional to the cube of the intensity, the integrated far-field answers are weighted by $I^{3}$; the spatially integrated instantaneous intensity (black) and spectral shifts (red) are simultaneously displayed. The $\pm \sigma_{S T D}$ standard deviation in the quantities from the spatial non-uniformities are indicated by the shaded areas (gray and pink respectively) to indicate the range of the measurement our experiment would have observed. Also shown are the near-field estimates of Doppler shifting (cyan), relativistic self-phase modulation (SPM, green) and their combined effect (Total, blue) are linearly weighted by the local intensity on target, and hence energy. In either case, the spatially integrated, intensity weighted shifts (red) are reasonably bound by the near-field Doppler (cyan) and combined Doppler/SPM curves (blue) which, in contrast to previous work, would suggest the SPM is

\footnotetext{
${ }^{31}$ These figures have been convolved using equivalent finite spatial and temporal resolution to the experimental measurement, averaged over a $\sim 125 \mathrm{fs} \times \mathrm{f} / 15$ window.
} 

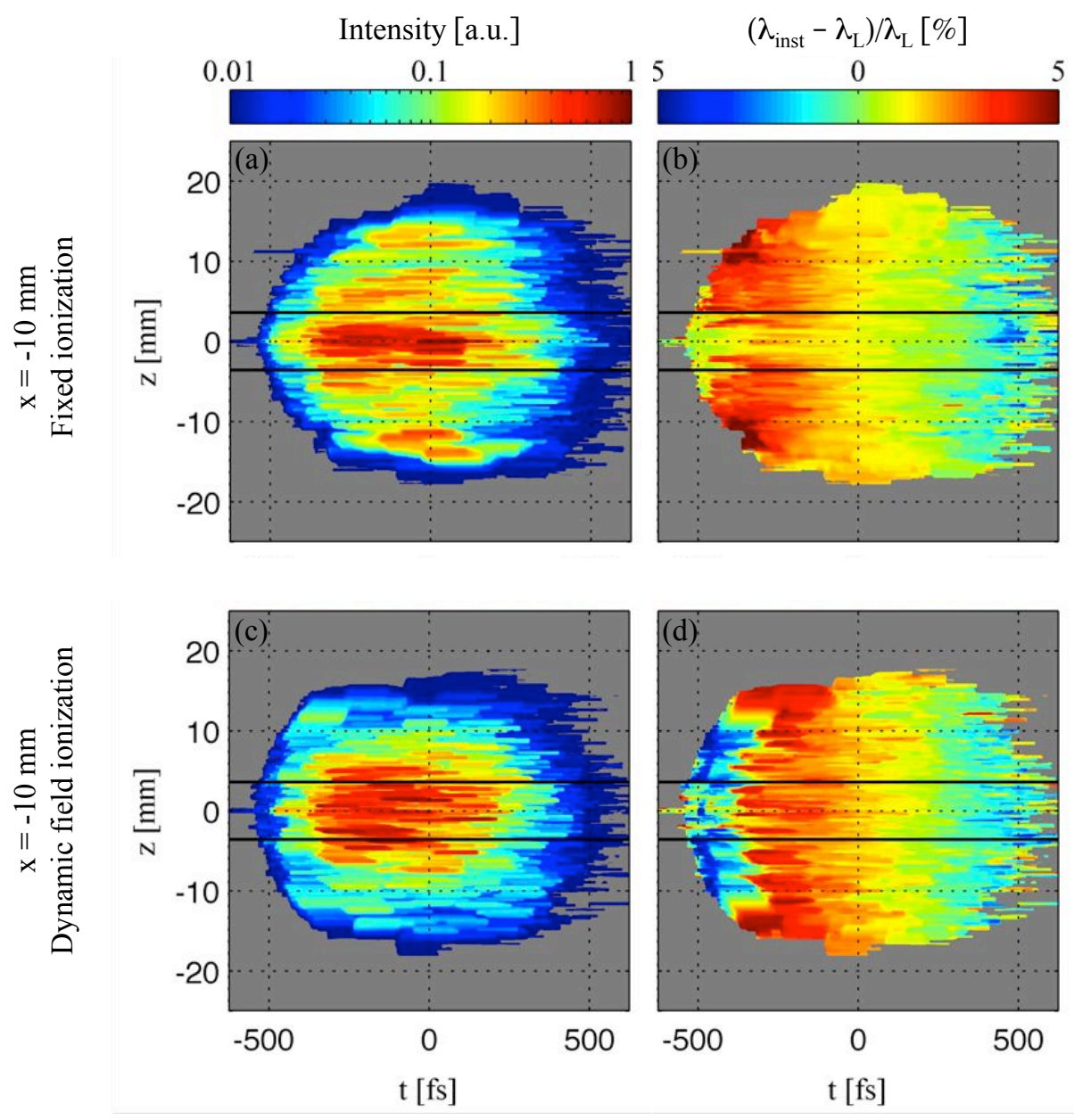

Figure 4.8: (Color) Spatially and temporally resolved far-field (left column) intensity and (right column) instantaneous spectral shifts of the specularly reflected pulse for the (first row) fixed and (second row) dynamically ionizing simulations at $x=-10 \mathrm{~mm}$. Also shown are is the spatial acceptance window of the experimental measurement (black).

playing some role in these interactions.

Any modifications that are sensitive to the spatial and temporal intensity distribution, however, will be quantitatively wrong as simplified temporal $\left(\sin ^{2}\right)$ and spatial (Gaussian) profiles were assumed for convenience; any structure in these experimental quantities ${ }^{32}$, can give rise to drastically different characteristics in space, time and spectrum because of the highly non-linear nature of these relativistic phenomena. While it is clear that these pulses

\footnotetext{
${ }^{32}$ Rest assured, there are many.
} 

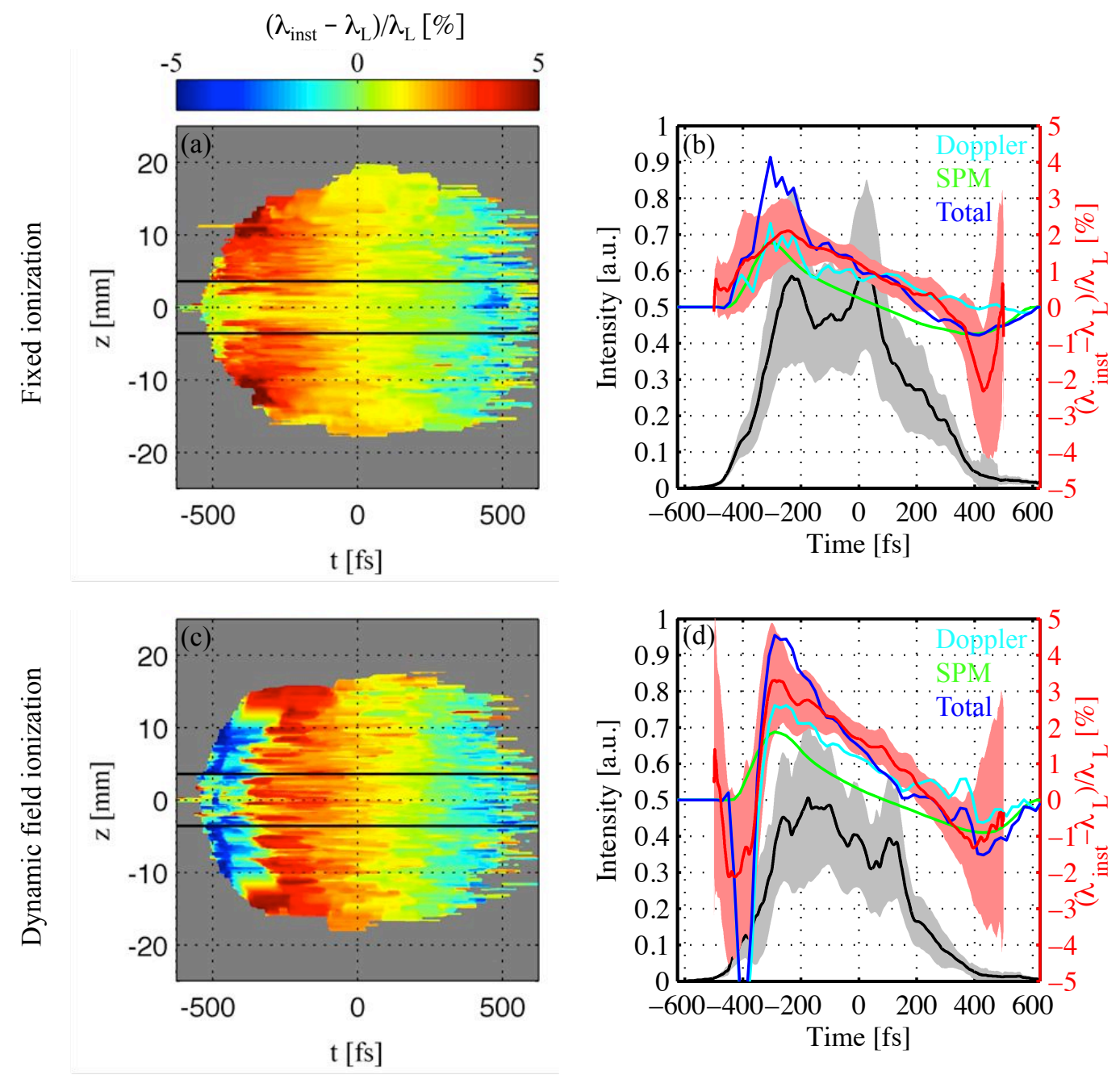

Figure 4.9: (Color) Near and far-field instantaneous spectral shifts. The top row shows the fixed ionization case and the bottom the dynamic ionization case with field ionization. The left column repeats the spatially and temporally resolved far-field spectral shifts from Figure 4.8 and the right column shows intensity weighted, spatially integrated lineouts of the intensity (black and gray) and instantaneous wavelength shifts (red and pink). Also shown are estimates of relativistic self-phase modulation (green), Doppler shifts (cyan) and their combined effect (blue) from near-field quantities.

aren't quantitatively characteristic of the experimental results, we find that the trends in spectral, spatial and temporal broadening are qualitatively similar. 


\subsection{Hot-electron properties}

The observation of an early (however weak) blueshift in the experimental data suggests that perhaps ionization is influencing the specular pulse measurements. Ultimately, electrons are the end-product of interest in these experiments for the applications previously discussed so the question remains: do ionization phenomena in the under-dense plasma result in observable differences in the hot-electron populations produced in these interactions?

Shown in Figure 4.10 are the spatially and temporally integrated electron energy spectra (on a $\log _{10}$-linear scale) for the fixed ionization (dashed - blue) and field ionization (blue) simulations. ${ }^{33}$ Although similar, the spectra differ slightly between 1 and $10 \mathrm{MeV}$ where the field ionization case showed higher coupling into $\sim U_{p}^{T W}$ electrons (indicated by the vertical dashed-black line). The overall total hot-electron coupling into the bulk of the target, defined as all electrons with energy above $50 \mathrm{keV}$ that pass through the diagnostic plane $5 \mu \mathrm{m}$ deep inside solid density, was $42.3 \%$ and $55.2 \%$ of the incident laser energy for the fixed and field ionization cases respectively.

Previous studies of hot-electron generation in the presence of short scale length preplasmas $[58,66]$ have observed that the energy distributions can be strongly Wilks-like [34], having Boltzmann-like distributions with temperature approximately equal to the travelingwave quiver energy $\left(U_{p}^{T W}=1.1 \mathrm{MeV}\right.$ in this case, indicated by the red curve). While a reasonable approximation for the electron energy distribution around $U_{p}^{T W}$, the distributions appear to have multiple components with one being significantly hotter which dominates the spectra above a few $M e V$. Many mechanisms for generating these supra-thermal electrons have been proposed [142-144] but, from the discussion in Chapter 1.5, we can see that plane-wave accelerations are completely capable of generating $>70 \mathrm{MeV}$ in these fields (Figure 1.4) given a hot enough plasma and sufficient acceleration distance. This is supported by previous work by Krygier [145], which suggests that these electrons are merely characteristic of longer plane-wave acceleration distances from a process they refer

\footnotetext{
${ }^{33}$ These electrons were recorded $5 \mu \mathrm{m}$ beyond the solid density at $x=10 \mu \mathrm{m}$ because of the dimpled surface. This is to help facilitate future comparisons of the hot-electron source between the high and lowcontrast simulations.
} 


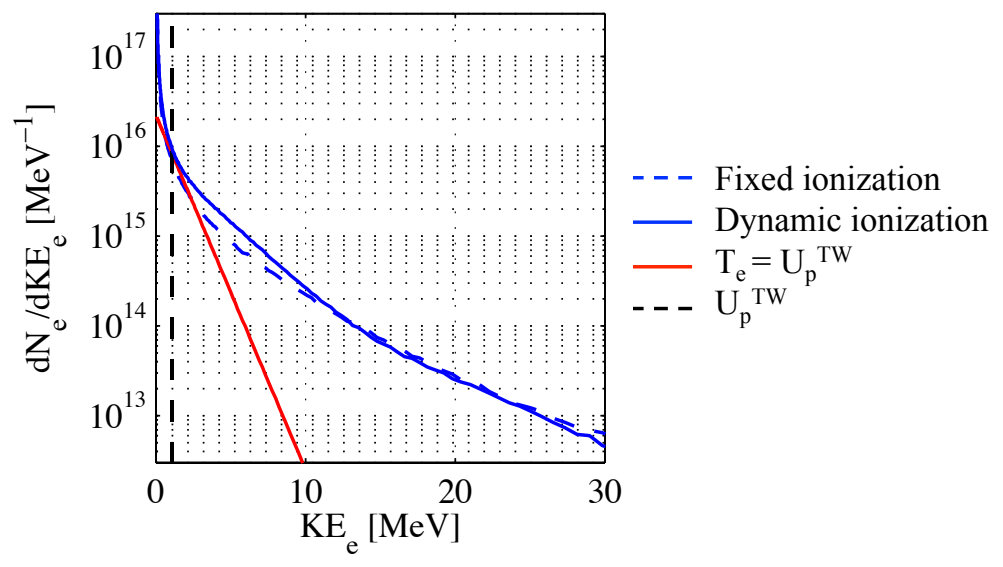

Figure 4.10: (Color) Spatially and temporally integrated hot-electron energy spectra for the fixed ionization (dashed-blue) and field ionization (blue) simulations. Also shown is the quiver energy (dashed-black) and a Wilks-like distribution for this pulse (red).

to as LIDA, or loop-injected direct acceleration. In this situation, we would expect that the angular distributions would be characteristic of the classical ejection angle (Equation 1.6).

The presence of free electrons in the under-dense plasma, where the laser can readily propagate, suggests that the angular distributions would be similar to those in Figure 1.4, with a clearly defined bifurcation (at least for the higher energy electrons). In a similar fashion, Figure 4.11 shows the spatially and temporally integrated angular distributions for the (a) fixed ionization and (b) dynamic field ionization simulations; they are resolved by kinetic energy (integrated over a $20 \mathrm{fs}$ temporal window) and each energy bin is individually normalized to show how the shape of the distribution changes with energy. For reference, the classical ejection angle (dashed-white) and $U_{p}^{T W}$ (dashed-black) are shown. The angular distributions are similar below $U_{p}^{T W}$, at which point the distribution is at its broadest. Even higher energy electrons have quite asymmetric angular distribution, preferentially cho osing one orientation over the other. If these higher energy electrons were solely characteristic of the plane-wave acceleration, we would expect the distributions to be partially bifurcated so something else is clearly at work here. Additionally, this influence must manifest itself differently between the fixed and dynamically ionizing cases. 


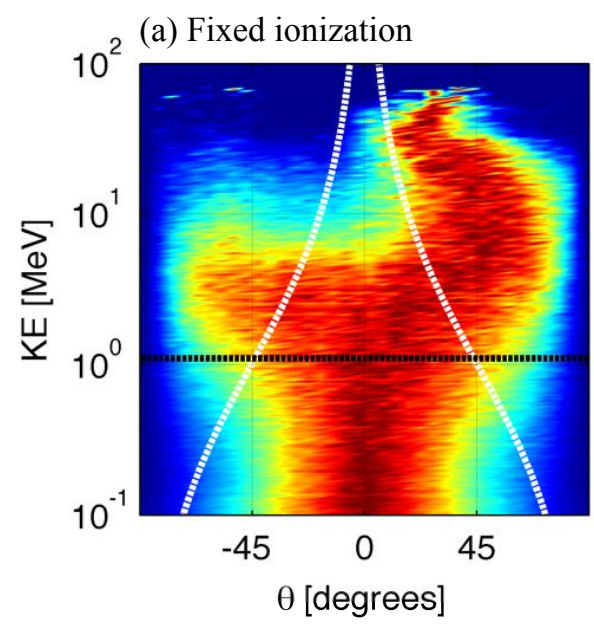

(b) Dynamic field ionization

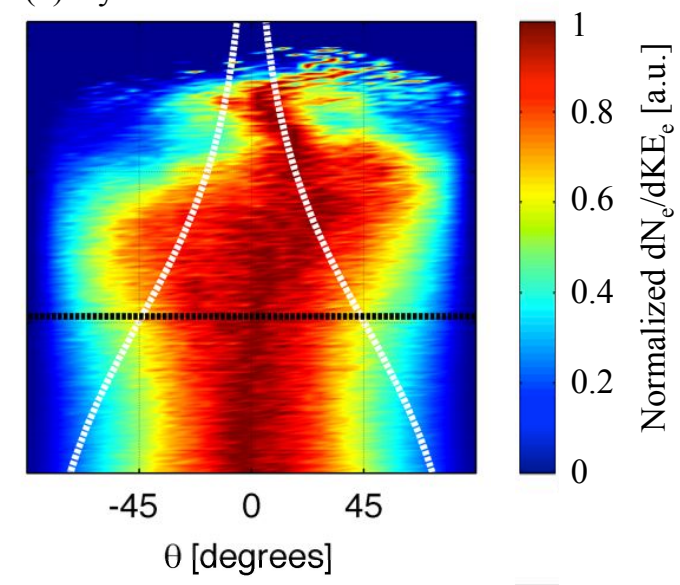

Figure 4.11: (Color) Spatially and temporally integrated hot-electron angular distributions, resolved by kinetic energy, for the (a) fixed ionization and (b) dynamic field ionization simulations.

At early times, neither the broadening angular distributions of the $\sim 1 \mathrm{MeV}$ electrons or the asymmetry in the higher energy electrons is present. In fact, these deviations from the expected results only begin to appear when the rising half-peak of the pulse reaches the target. Before this time, electron angular distributions are quite well bounded by the classical ejection angle. Beyond this time, the evolution of quasi-static fields in the underdense plasma were found to evolve quite differently in the two simulations, shown in Figure 4.12 for the (a) fixed ionization and (b) dynamically ionizing cases at the rising half-peak, peak, and falling-half peak intensities upon reflection at the critical surface. ${ }^{34}$

By the time the rising half-peak intensity reflects off the critical surface, quasi-static magnetic fields in the under-dense plasma region near the critical surface have grown to $\sim 100 M G$ levels, extended over $\sim 1 \mu \mathrm{m}$ in diameter. From the calculations in Figure 1.10, the fields present at the rising half-peak of the pulse is sufficient to completely trap anything below $\sim 3 \mathrm{MeV}$. Any electrons below this threshold will be completely trapped and trajectories of electrons significantly above will only be slightly perturbed, but $\sim 3 \mathrm{MeV}$

\footnotetext{
${ }^{34}$ These fields were estimated by spatially averaging over a laser wavelength instead of the conventional laser period in time. At this time, LSP doesn't have this time-averaging feature for the fields quantities.
} 
electrons are in the 'Goldilocks zone' resulting in significant trajectory perturbations which broadens the angular distrubtion. The highest energy electrons, generated in the most intense portions of the pulse, likely originate inside the strongest laser filaments where self-focusing has produced intensities several times higher than in vacuum. Interestingly, the filaments appear to exist in regions with nearly uniform quasi-static magnetic fields. Although not verified with trajectories (at this time), this is likely why the most of these high energy hot-electrons preferentially veer to one side and not the other.

Clearly, the evolution of these fields is quite different between the two cases where fields with the fixed ionization are significantly more pronounced than for the field ionization case, resulting more divergent hot-electrons. Since the most of the generation mechanisms discussed in Chapter 1.5 are proportional $\nabla n_{e}$, it is possible that the increased electron density from field ionization could lessen this gradient and lead to slower field growth. 
(a) Fixed ionization
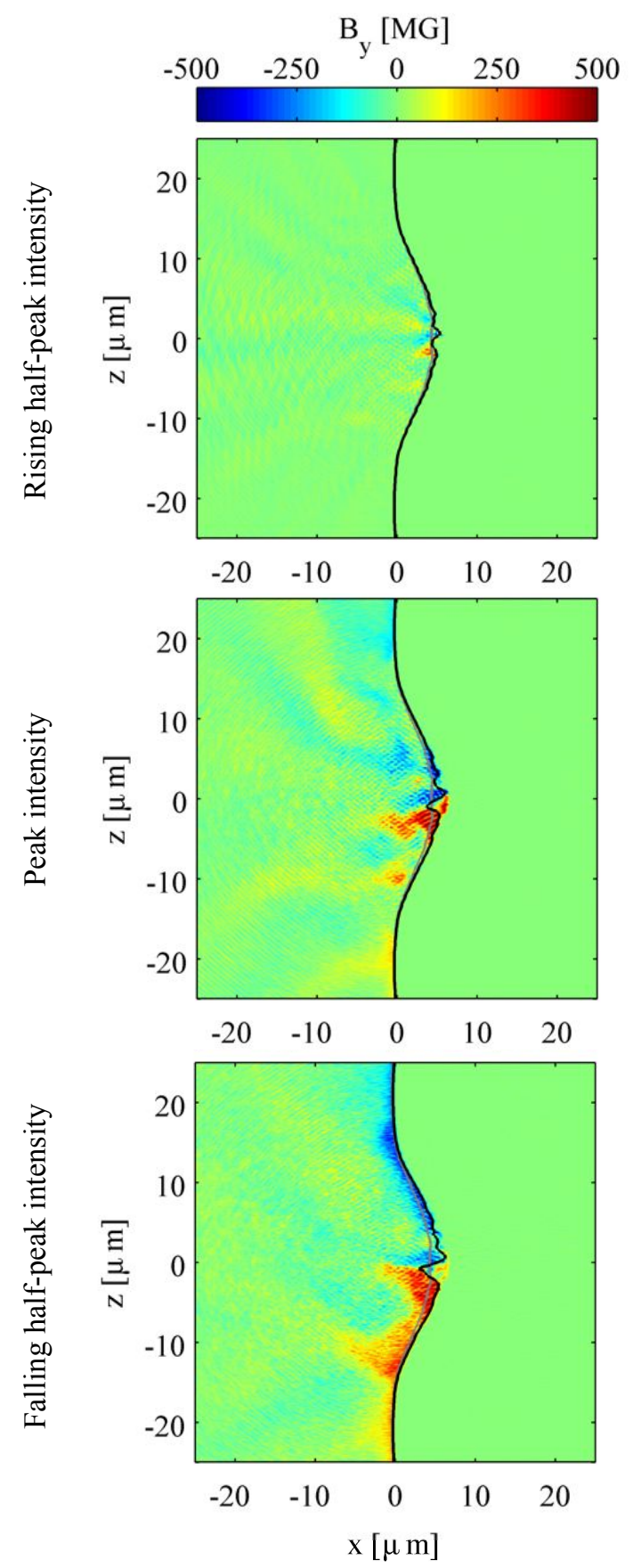

(b) Dynamic field ionization
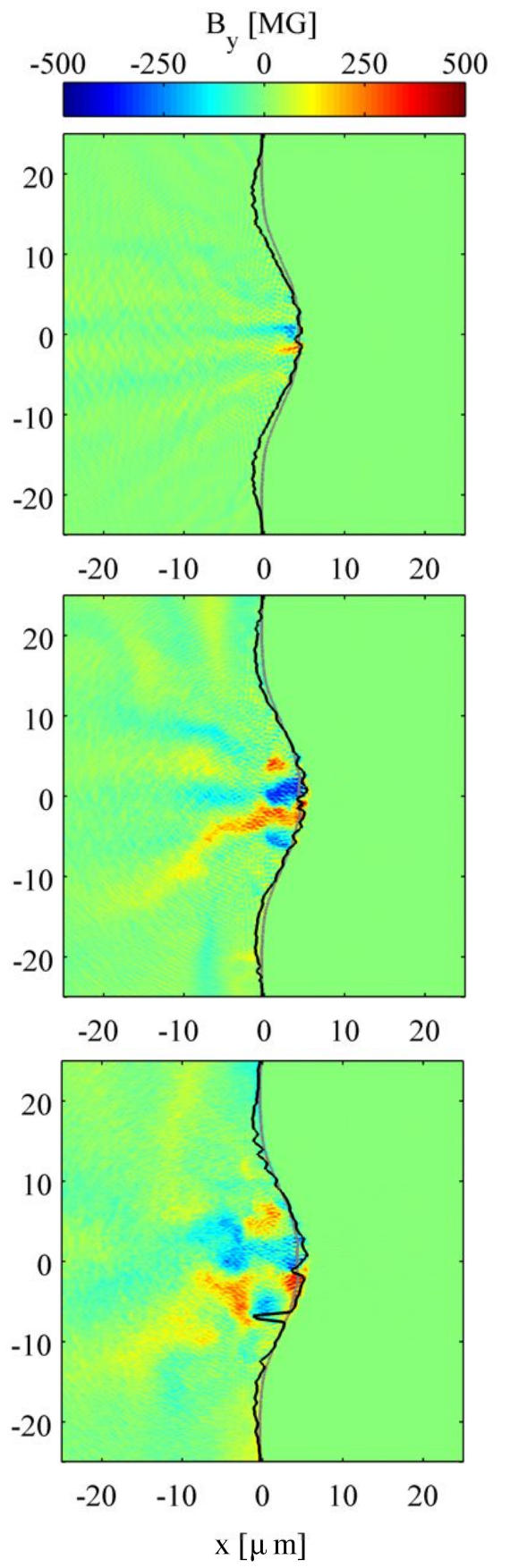

Figure 4.12: (Color) Evolution of the quasi-static magnetic fields generated in the underdense pre-plasma near the relativistic critical surface (black). Also shown in gray is the original relativistic critical surface. On the left, (a) shows the fixed ionization case and on the right (b) shows the simulation results from the dynamic field ionization. 


\subsection{Summary}

With side-by-side comparison of fixed and field ionization simulations, we find that even the modest changes in electron densities from field ionization that occurs in this under-dense aluminum pre-plasma can have some very profound effects on laser propagation, quasi-static field generation and the resulting hot-electron acceleration. Perhaps most importantly, the influence of ionization on the temporally resolved spectral properties of the specularly reflected pulse should be observable in an experiment (with a FROG). Since the interaction region dynamically evolves, far-field measurements of the pulse can be sensitive to spatial non-uniformities but integration over the entire far-field pulse shows strong correlations to the near-field physics. Due to a variety of simplifying assumptions made of the incident laser's spatial and temporal envelope properties, the resulting far-field quantities were quite different from the experimental results. However, we do find that all the qualitative trends of spatial and spectral broadening are reproduced as well as the temporally resolved shifting. Since the shifting magnitudes are similar to the experiment, it is reasonable to believe that the field ionization and pe-plasma electron density profile steeping from field ionization and Doppler motion of the critical surface, respectively, are characteristic of the experimental conditions. Hot-electrons generated in such an experiment could be strongly influenced by the growing quasi-static magnetic fields, resulting in preferential electron directionality and broader angular distributions compared to the idealized plane-wave accelerations discussed previously. 


\section{Chapter 5}

\section{COUPLING OF LASER ENERGY INTO HOT-ELECTRONS IN HIGH-CONTRAST RELATIVISTIC LASER-PLASMA INTERACTIONS}

As previously discussed, extensive under-dense preformed plasma can develop around the laser interaction region before the main pulse arrives due to pre-pulse in low-contrast pulses. This pre-plasma, due to a variety of non-linear phenomena, can drastically modify the incident laser pulse as it propagates through the plasma, before ultimately being reflected at the relativistic critical surface. Correspondingly, hot-electrons generated under such conditions can be less predictable and desirable depending on the application of interest. Using highcontrast pulses can remove some of these effects and create a more repeatable interaction, but often at the cost of less overall coupling and increased sensitivity to initial target surface conditions. In this Chapter, which was previously published as Coupling of laser energy into hot-electrons in high-contrast relativistic laser-plasma interactions [Physics of Plasmas, 20(3):033104, 2013] [146], we ${ }^{35}$ discuss a study on high-contrast laser-plasma interactions using a systematic parameter study of highly simplified sinusoidal surface perturbations. In particular, we address hot-electron generation from sharp interfaces and consider the overall effect of surface perturbations on the resulting distributions.

\footnotetext{
${ }^{35}$ I exclusively performed all of the simulation and analysis work in this thesis but only after many useful discussions and suggestions from the co-authors.
} 


\subsection{Abstract}

We use particle-in-cell simulations to explain the mechanisms responsible for the coupling of laser energy into relativistic electrons for the case of sharp interface, solid density metal targets free of pre-plasma. For perfectly flat interfaces, the accelerated electron trajectories are dominated by the standing-wave field structure formed by interference between incident and reflected pulses. We find that quasi-static magnetic fields that develop near the interface play only a minor role in perturbing the relativistic electron trajectories but can contribute to enhanced absorption. Target surfaces that are structured exhibit enhanced absorption and the acceleration mechanism deviates from the clean standing-wave acceleration mechanism leading to more stochastic electron heating and larger divergence angles.

\subsection{Introduction}

Ultra-intense $\left(>10^{18} \mathrm{~W} / \mathrm{cm}^{2}\right)$ laser-plasma interactions are capable of producing relativistic electric beams with a variety of applications including the fast-ignitor approach to inertial confinement fusion [1], creating ultra-short x-ray sources [3] and isochorically heating matter to warm-dense states [2]. Each application has specific requirements on energy spectrum, angular distribution and conversion efficiency where control of various aspects of these relativistic electron distributions is essential to their effectiveness. Ultra-intense short-pulse lasers are typically preceded by several $m J s$ or more of laser energy nanoseconds before the main pulse arrives at the target. This 'pre-pulse' usually has sufficient intensity to create $10 \mathrm{~s}$ of microns of under-dense plasma in front of the target. Not only does this move the laser interaction interface from supra-critical solid density to the lower relativistic critical density, it also subjects the main pulse to instabilities [47, 58-60, 132] and quasi-static field generation $[45,46]$ which can greatly modify relativistic electron generation and transport.

High-contrast lasers minimize the laser energy before the main pulse and therefore limit pre-plasma formation. Several facilities, like LULI [47], Trident [48], HERCULES [49] and Titan already have this pulse cleaning capability typically obtained through nonlinear optical processes, such as harmonic generation [] and third order cross-polarized wave generation 
[54], or with plasma mirrors $[55,56]$. Under these conditions, the interaction region more closely resembles the initial target interface thus making surface conditions important. The advent of laser systems with intense, but extremely clean, laser pulses has ushered in a new regime of experiments including, particularly, the ability to use very thin (sub-micron) targets for ion acceleration via Target Normal Sheath Acceleration (TNSA) [61, 147]. However, the absence of pre-plasma tends to reduce the laser-coupling efficiency and, in general, the coupling mechanisms in this regime are not well understood. This is the subject of our study.

Coupling of laser energy into electrons always requires two stages: a mechanism for getting electrons into the laser field and another for them to de-phase from the laser. For the case of a laser interacting with a sharp interface, the difficulty in coupling arises from getting electrons out of the supra-critical density plasma and into the intense laser fields. In ultra-intense interactions, absorption is dominated by collisionless processes [85, 90] and several heating mechanisms have been suggested. The Brunel effect [100] relies on a component of the laser electric field being normal to the interface that can push and pull electrons across the vacuum interface but is only present for p-polarized lasers at non-normal incidences. Alternatively, relativistic $\vec{j} \times \vec{B}$ heating [101] can exist at normal incidence as it relies on the oscillating component of the ponderomotive force to drive electrons across the interface but the driving longitudinal electric field strength decays exponentially with distance inside solid density.

For perfectly flat interfaces irradiated at normal incidence, low coupling results in a Standing-Wave (SW) field pattern near the interface from the interference between the incident and reflected waves. Electrons that eventually reach vacuum are accelerated with trajectory characteristics unique to this standing-wave structure. For the perfect conductor discussed by May et al [102], only electrons inside the plasma with sufficient transverse momentum (approaching $m_{e} c$ ) are able to reach vacuum because of the strong surface magnetic fields. Once in vacuum, electrons are accelerated by the electric field parallel to the surface (peaked at $\lambda_{L} / 4$ away from the interface) and then turned back into solid density by the magnetic field. This acceleration mechanism differs from both Brunel and relativistic 
$\vec{j} \times \vec{B}$ heating in that no electric field component normal to the surface is required. However, it is not present in plasmas with electron energies below a few $10 \mathrm{~s}$ of $\mathrm{keV}$ and therefore cannot address how initially cold targets can reach this state.

Considerable attention has also been paid to the current driven Weibel-like quasi-static magnetic fields near the interface which have been assumed to be responsible for everything from additional electron dephasing from the laser and more randomized electron trajectories [72] to clearly structured bifurcations in electron angular distributions [89]. While these effects are known, little work has been done that isolates the relative influences of the two phenomenon or the resulting distributions of the hot-electrons that propagate deep inside solid density.

Eventually, perturbations in the initially flat interface grow as these Weibel-like fields develop near the interface [98] with magnitude and extent that can even affect the directionality of relativistic electrons. These perturbations can increase absorption by introducing a component of the laser electric field that is locally normal to the surface. Together, surface perturbations and enhanced coupling can significantly modify the standing-wave field pattern.

For targets with initial surface perturbations, significant enhancements in absorption have been reported which have typically been attributed to enhanced Brunel [72, 98], resonant excitation of surface plasma waves [117-120] or local field enhancement via Mie resonance $[121,122]$ depending on the specifics of each study. However, the dynamic competition between heated plasma expansion and laser profile steepening can drive changes in the electron density profile [35]. Plasma expansion that cannot be overcome by the laser profile steepening can lead to smoothing over of the absorption enhancing perturbations by the motion of the relativistic critical density surface and the development of an underdense plasma shelf though which the laser must propagate. However, increased electron populations in under-dense regions can still result in enhanced coupling.

The resulting lower reflectivity creates a laser field structure more closely resembling that of the propagating incident laser pulse (in vacuum), referred to here as a TravelingWave (TW). In general, because of spatial non-uniformities in laser reflectivity, electro- 
magnetic fields near the interface of structured targets will have a complicated superposition of standing-wave and traveling-wave components. The processes by which electrons gain energy in these field structures are fundamentally different from each other and their relative contributions are apparent in the accelerated electron trajectory characteristics. Traveling-wave accelerations in under-dense regions, not associated with the sharp interface acceleration mechanisms, can contribute a significant fraction of the total conversion efficiency. Furthermore, the complicated field structure can provide an additional dephasing mechanism by which electrons can gain energy stochastically [142-144].

Using $2 D 3 V$ particle-in-cell simulations (described in Section 5.3) with an initially sharp, solid density interface (free of pre-plasma), we study hot-electron generation in the context of high-contrast relativistic laser-plasma interactions using $100 \mathrm{fs}$ pulses. For perfectly flat targets irradiated at normal incidence, we show how even cold targets $\left(T_{e}=5 \mathrm{eV}\right)$ can produce relativistic electrons by including the effect of skin depth on the near-surface standing-wave field structure in Section 5.4. In Section 5.5, we discuss coupling and accelerated electron characteristics from targets that have surface perturbations. We show Weibel-like quasi-static magnetic fields near the interface do not significantly contribute to the directionality of relativistic electron trajectories but can enhance absorption when surface structures are present. With under-dense plasma shelf formation, we find that a significant fraction of the electron energy can come from traveling-wave accelerations and not just the standing-wave driven interactions. While solid density, quasi-static magnetic fields can affect even relativistic electron trajectories, we show that the randomization of accelerated electron trajectories and angular distribution broadening is more consistently associated with wave-front perturbations. Finally, in Section 5.6, we conclude with some speculations on shorter and longer pulse behavior.

\subsection{Simulation setup}

$2 \mathrm{D} 3 \mathrm{~V}$ Cartesian fully kinetic particle-in-cell simulations were performed to determine the effect of surface perturbations on absorption using the commercially available PIC code 


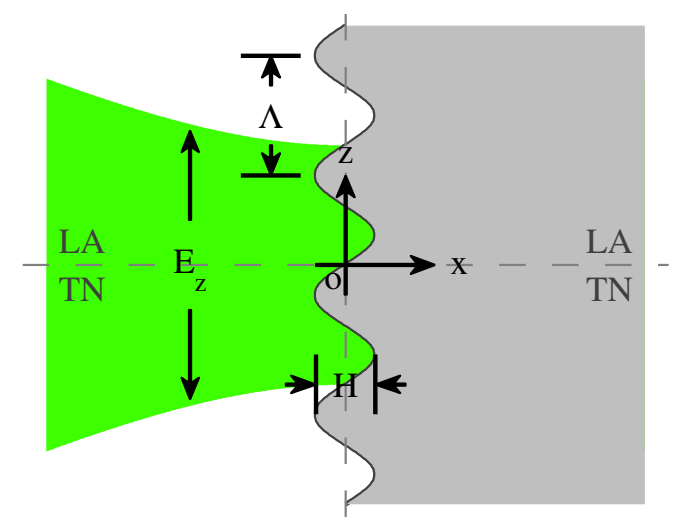

Figure 5.1: (Color) PIC simulation geometry for sinusoidal surface features. The laser (polarized along the $z$-axis) is normally incident propagating from left to right where LA $(\mathrm{TN})$ is the Laser-Axis (Target-Normal). The laser focal plane and (mean) surface interface is located at $x=0 \mu \mathrm{m}$. Sinusoidal surface perturbations have spatial periodicity $\Lambda$ and peak-to-trough height $H$.

Table 5.1: Conversion efficiency of surface features addressed in this study.

\begin{tabular}{cccc}
\hline \hline Surface & $\begin{array}{c}\text { Height } \\
\mathrm{H}\left[\times \lambda_{L}\right]\end{array}$ & $\begin{array}{c}\text { Wavelength } \\
\Lambda\left[\times \lambda_{L}\right]\end{array}$ & $\begin{array}{c}\text { Conversion Efficiency } \\
\eta_{L \rightarrow e^{-}}[\%]\end{array}$ \\
\hline (a) & 0 & - & 4.6 \\
(b) & $1 / 8$ & 1 & 21 \\
(c) & 1 & 1 & 41 \\
(d) & 1 & 2 & 47 \\
\hline \hline
\end{tabular}

LSP [104]. For simplicity, sinusoidal features were chosen to represent initial target surface roughness with various peak-to-trough heights $H$ and spatial periodicity $\Lambda$ (geometry outlined in Figure 5.1 where $L A / T N$ is the Laser-Axis/Target-Normal). The target was modeled as a solid density $\left(n_{e}=1.81 \times 10^{23} \mathrm{~cm}^{-3}\right)$, initially $Z=3$ charge state Aluminum slab, $25 \mu m$ deep by $50 \mu m$ wide with $25 \mu m$ of vacuum in front of the target to accommodate electron refluxing (with absorbing boundary conditions). Hot-electron properties (time, energy and angle) are recorded as they pass through a diagnostic plane at $x=5 \mu \mathrm{m}$ inside solid density where $x=0 \mu m$ corresponds to the (mean) solid density interface and incident laser focal plane (in vacuum). 
The incident laser pulse (i.e traveling-wave with wavelength $\lambda_{L}=527 \mathrm{~nm}$, period $T_{L}=1.76 \mathrm{fs}$, corresponding to a non-relativistic critical density $n_{c}=m_{e} \epsilon_{o} \omega_{L}^{2} / e^{2}=4.0 \times$ $10^{21} \mathrm{~cm}^{-3}$ ) has a $200 \mathrm{fs}$ duration $\sin ^{2}$ intensity profile (100 $\mathrm{fs}$ FWHM) incident normally on the target with an $8 \mu \mathrm{m}$ focal spot FWHM, polarized in the simulation plane, with a cycle averaged peak intensity of $4.65 \times 10^{19} \mathrm{~W} / \mathrm{cm}^{2}$. The quiver energy $U_{p}$ of electrons in the oscillating electric field is given by $U_{p}^{T W}=\left(\gamma_{o}^{T W}-1\right) m_{e} c^{2}=1.1 \mathrm{MeV}$ where $\gamma_{o}^{T W}=\sqrt{1+a_{o}^{2}}=3.2$ is the relativistic Lorentz factor and $a_{o}=e E_{o} / m_{e} \omega_{L} c=3.1$ is the normalized vector potential (where the ${ }^{T W}$ superscript refers to the vacuum value travelingwave field structure).

The simulation was performed using a direct implicit algorithm [104] incorporating an energy-conserving particle push. The grid had a spatial resolution of $31.2 \mathrm{~nm}\left(\sim \lambda_{L} / 16\right)$ and temporal resolution of 27.5 as $\left(\sim T_{L} / 64\right)$. Through a separate study, hot-electron distributions in time, space, energy and angle were found to be sufficiently converged at these resolutions. Electron macro-particle densities ranged from 144 to 196 per cell and the ions ranged from 25 to 49 per cell resulting in $\sim 200 M$ macro-particles in the simulation. All electron and ion species were fully kinetic and collisionless with initial electron and ion temperatures of $5 \mathrm{eV}$. Sequential tunneling field ionization was included using ADK (Ammosov, Delone and Krainov) ionization rates for ions in an alternating electromagnetic field [87].

Four surface perturbation profiles have been chosen to illustrate different absorption phenomenon in high-contrast interactions. They are summarized in Table 5.1 (consistently labeled throughout as (a) through (d)) along with $\eta_{L \rightarrow e^{-}}$, the total conversion efficiency of laser energy into hot-electrons. In this context, hot-electrons refer to electrons with kinetic energy greater than $50 \mathrm{keV}$ that pass through the diagnostic plane at $x=5 \mu \mathrm{m}$ deep inside solid density. Surface (a) is the simplest surface with a perfectly flat interface. Surface (b) has, by most definitions, optically flat surface perturbations with $H=\lambda_{L} / 8, \Lambda=\lambda_{L}$ $\left(\sim \lambda_{L} / 25 R M S\right)$. The larger surface perturbations in surfaces (c) $H=\lambda_{L}, \Lambda=2 \lambda_{L}$ and (d) $H=\lambda_{L}, \Lambda=\lambda_{L}$ have been selected to isolate the separate influences of wavefront perturbations and solid density magnetic fields on hot-electron generation and transport. 


\subsection{Perfectly flat interface}

The simplest sharp interface is one that is perfectly flat, surface (a). The evolution of the electron density profile $\left(\log _{10}\left(n_{e}\right)\right)$ is shown in Figure 5.2(i) at three instances in time: the rising half-peak, peak and falling half-peak intensities of the pulse upon reflection. Also shown are the original solid density interface (dashed black line) and the current relativistic critical density interface (solid black line). Throughout the duration of the run, the interface remains sharp only becoming slightly rippled at late times and the reflectivity remains quite high averaging $\sim 85 \%$.

High reflectivity and a flat reflecting surface form a standing-wave field pattern due to the interference between the incident and reflected pulses. However, since our collisionless supra-critical plasma isn't a perfect conductor, the fields inside the plasma aren't zero everywhere but instead fall off exponentially with distance from the interface. The field structure near the plasma interface can be characterized by (for the same geometry as the simulation) [85]

$$
\begin{gathered}
E_{z}(x, t)= \begin{cases}2 E_{o} \sin \left(k_{L} x+\phi\right) \sin \omega_{L} t & x<0 \text { (vacuum) } \\
E(0) \exp \left(-x / l_{s}\right) \sin \omega_{L} t & x \geq 0 \text { (solid) }\end{cases} \\
B_{y}(x, t)=\int \partial_{x} E_{z}(x, t) d t= \begin{cases}-\frac{2 E_{o}}{c} \cos \left(k_{L} x+\phi\right) \cos \omega_{L} t & x<0 \text { (vacuum) } \\
\frac{E(0)}{l_{s} \omega_{L}} \exp \left(-x / l_{s}\right) \cos \omega_{L} t & x \geq 0 \text { (solid) }\end{cases}
\end{gathered}
$$

where $k_{L}=2 \pi / \lambda_{L}, E_{o}$ is the vacuum amplitude of the incoming laser field, $l_{s}=c / \omega_{p}(1-$ $\left.\omega_{L}^{2} / \omega_{p}^{2}\right)^{-1 / 2}$ is the collisionless skin depth (with plasma frequency $\omega_{p}^{2}=n_{e} e^{2} / m_{e} \epsilon_{o}$ ) and $\phi$ is the phase offset of the standing-wave due to the plasma response. From Faraday's Law, components of $\vec{E}$ parallel to the surface (i.e. $E_{z}$ ) must be continuous across the interface. This boundary condition lets us solve for $E(0)$ and $\phi$ as follows

$$
\begin{aligned}
& E(0)=-2 E_{o} \frac{\omega_{L}}{\omega_{p}} \\
& \tan \phi=-l_{s} \frac{\omega_{L}}{c} .
\end{aligned}
$$




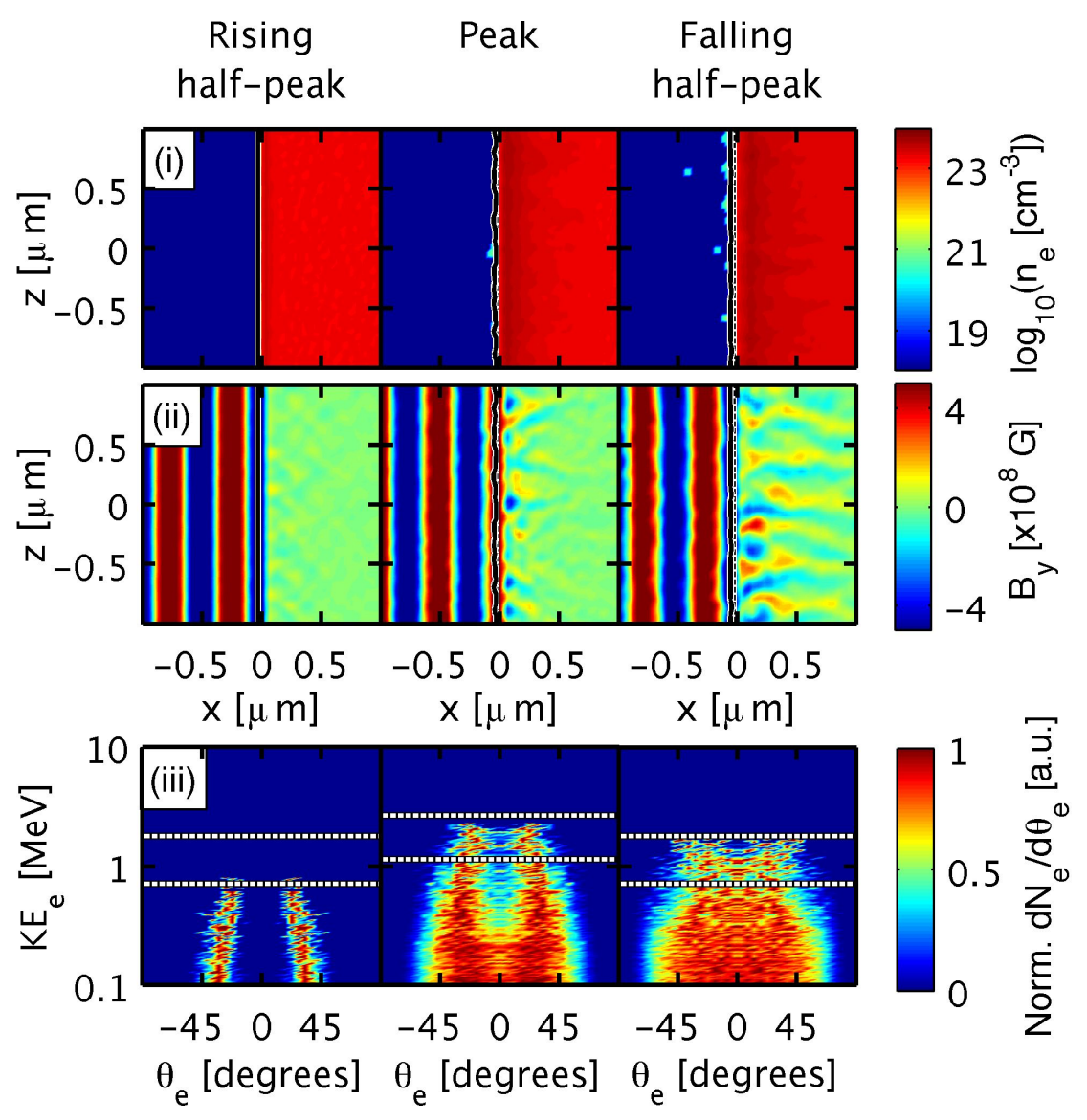

Figure 5.2: (Color) Temporal evolution perfectly flat interface, surface (a) with (i) the electron density (log10), (ii) By (linear) and (iii) angular distributions (normalized) resolved by energy. The first column is a snapshot in time at the rising half peak, the middle column at the peak of the pulse and the third the falling half peak. Vertical solid(dashed) black curves in (i) and (ii) are relativistic(original) surface and the horizontal dashed white lines in (iii) indicate $U_{p}^{T W}$ (lower) and $U_{p}^{S W}$ (upper).

In the perfect conductor limit $\left(l_{s} \rightarrow 0\right)$, the perfect standing-wave structure is recovered where the magnetic field is peaked and the electric field is always zero at the interface where $E_{z}$ peaks at exactly $\lambda_{L} / 4$ away from the surface at twice the vacuum value, $2 E_{o}$. Correspondingly, the peak normalized vector potential is also twice the vacuum travelingwave value yielding relativistic Lorentz factor $\gamma_{o}^{S W}=\sqrt{1+\left(2 a_{o}\right)^{2}}=6.5$ and quiver energy $U_{p}^{S W}=\left(\gamma_{o}^{S W}-1\right) m_{e} c^{2}=2.8 \mathrm{MeV}$ (where the ${ }^{S W}$ superscript refers to the standing-wave field structure). 
For our solid density aluminum plasma target, the electric field peak is closer to the interface and finite electromagnetic fields persist at supra-critical densities over several skin depths from the interface as can been seen in the corresponding magnetic fields $\left(B_{y}\right.$, normal to the interaction plane) in Figure 5.2(ii). They are shown on a linear color scale to resolve the $500 M G$ quasi-static magnetic fields at supra-critical densities (right of the solid black curves). The standing wave structure is evident in the under-dense regions, as well (left of the solid black curves). The electromagnetic field is dominated by the laser field in under-dense regions and by quasi-static magnetic fields beyond a skin depth inside solid density.

Magnetic field generation at supra-critical densities can come from many sources including hot-electron currents [44], radial thermal transport [45] (i.e. thermoelectric $\propto \nabla T_{e} \times$ $\nabla n_{e}$ ) and DC currents in steep density gradients [46] (i.e. ponderomotive $\propto \nabla n_{e} \times \nabla I_{o}$ ). For this surface, magnetic fields inside solid density beyond a skin depth appear to be dominated by hot-electron currents. These highly anisotropic hot-electron currents inside solid density are susceptible to the Weibel-like two-stream electromagnetic instability [99] that causes these currents to filament resulting in $\sim 100 M G$ magnetic filaments extending over sub-wavelength spatial scales inside solid density. In some circumstances, these quasi-static magnetic fields have magnitude and extent that can significantly affect even relativistic electron trajectories and have reasonably been assumed to be responsible for more randomized accelerated electron trajectories in high-contrast laser-solid interactions $[72,89]$.

For the corresponding instances in time as the electron density and magnetic field snapshots (taking into account the time-of-flight to the diagnostic plane), the evolution of the angular distributions of hot-electrons as they pass through the diagnostic plane inside solid density is shown in Figure 5.2(iii). They are resolved by kinetic energy (integrated over a $10 \mathrm{fs}$ time window) and each energy bin is individually normalized to show how the shape of the distribution changes with energy. The horizontal dashed white lines indicate $U_{p}^{T W}$ and $U_{p}^{S W}$ (1.1 and 2.8 $\mathrm{MeV}$ respectively) and at all times the peak observed electron energy is between $U_{p}^{T W}$ and $U_{p}^{S W}$.

We observe a bifurcation in the angular distribution (which is especially clear at early 
times) that eventually blurs and fills in at later times similar to what was reported by Adam et al [89]. In contrast with Adam, we find that accelerated electrons throughout the entire interaction are characteristic of the standing wave structure that forms near the interface and not a result of growing quasi-static magnetic fields near the interface.

Shown in Figure 5.3 are phase-space representations of the hot-electron angular distribution, similar to Figure 5.2(iii), but instead from a calculation of test-particle electron trajectories in the above form of the standing-wave electromagnetic field. Perfect reflectivity was assumed and the half-peak intensity was chosen (corresponding to the first and third columns in Figure 5.2). Electron test particles were injected into the field as a point source at the solid density interface and sampled over an optical cycle. Only electrons that reached vacuum to get directly accelerated by the standing-wave are shown with their final kinetic energy and angle as they pass through the same diagnostic plane as the simulation $(x=5 \mu m)$. Again, $U_{p}^{T W}$ and $U_{p}^{S W}$ are indicated by the horizontal dashed white lines.

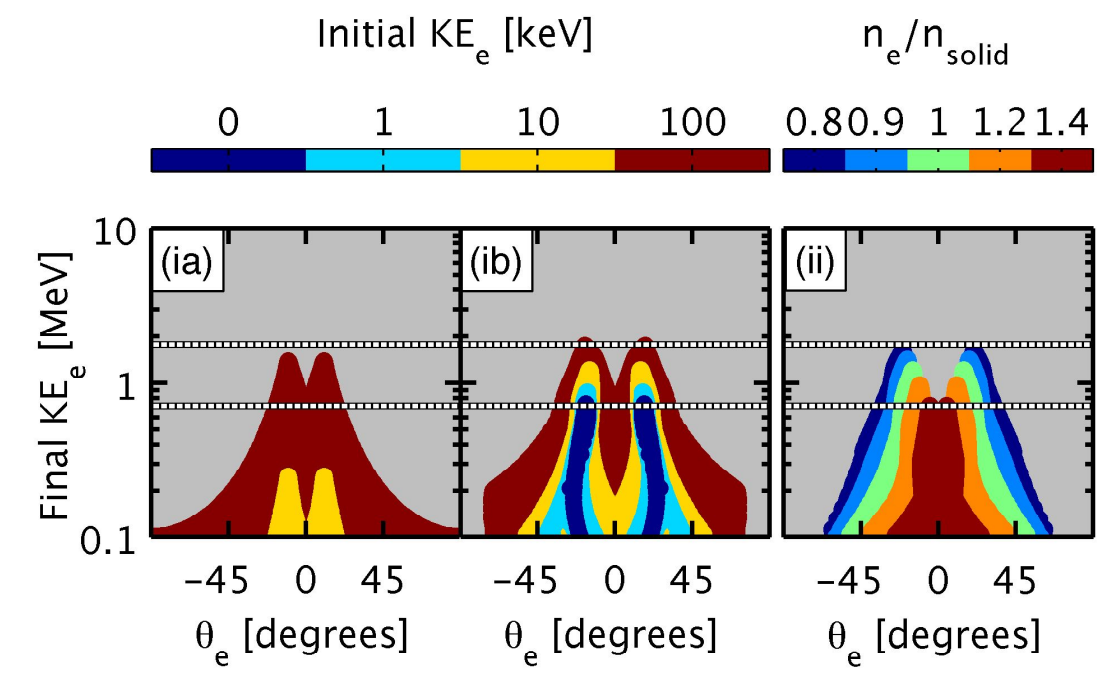

Figure 5.3: (Color) Hot-electron phase space calculations of the point source test particles accelerated from solid density by standing-wave EM fields as a function of final kinetic energy and angle (colored by initial kinetic energy) for (ia) a perfect conductor and (ib) plasma with the skin effect included. (ii) Initially $10 \mathrm{keV}$ electrons from different density interfaces (relative to initial solid density $n_{\text {solid }}$ at $45 n_{c}$ ). The horizontal dashed white lines indicate $U_{p}^{T W}$ (lower) and $U_{p}^{S W}$ (upper). 
The different colors in Figures 5.3(ia) and (ib) specify the initial electron kinetic energy and they outline the phase-space that can be filled by this point source. The final electron number density is not shown in this figure. Figure 5.3(ia) shows the perfect conductor case and, as discussed by May, only higher energy electrons (10s of $k e V$ ) can get accelerated to ponderomotive energies. The same initial electron energies are shown in Figure 5.3(ib) but now with the inclusion of the effect of the plasma skin depth on the fields (using the initial solid density). Clearly, electromagnetic fields within a skin depth are sufficient to inject even electrons that start from rest into the laser. Also, the peak observed electron energy and bifurcation 'finger' thickness are both sensitive to the initial electron energy.

These distributions are also sensitive to the standing-wave offset phase $\phi$ (and therefore electron density and relativistic effects). This is shown in Figure 5.3(ii) using only initially $10 \mathrm{keV}$ electrons but with varying target electron densities (relative to the initial solid density). With increasing density we find that both the angular separation between of the 'fingers' and peak electron energy decreases.

Clearly, the distributions we observe at early times in the simulation are much more consistent with the $\lesssim 1 \mathrm{keV}$ electrons accelerated by standing-wave fields that include the skin effect (dark and light blue in Figure 5.3(ib)) and not from a perfect conductor. This is consistent with the simulation in that, near the interface, the mean electron energy at the rising half-peak is less than $3 \mathrm{keV}$. In contrast with Adam, we find that the source of this bifurcation is clearly not from the Weibel-like filaments, as the hot-electrons that propagate inside solid density clearly keep the semblance of the standing-wave acceleration signature. This is especially clear at early times when the Weibel-like filaments aren't strong enough to influence electron trajectories and yet we still see a very clear bifurcation. By the time the falling half-peak of the pulse reflects, the bifurcation is no longer apparent. Indeed, the distribution begins to resemble the $<100 \mathrm{keV}$ electron phase space (dark red) in Figure 5.3(ib) consistent with the average electron energy near the interface being heated to $\sim 50 \mathrm{keV}$ as reported by the simulation: i.e. the filling in and broadening of the energyangle phase space is more consistent with target heating than trajectory randomization from Weibel-like fields as previously assumed. 


\subsection{Surface perturbations}

For the perfectly flat interface, the relativistic critical surface remains optically flat throughout the entire interaction only becoming slightly rippled near the end of the interaction. This rippling, likely due to the development of quasi-static Weibel-like fields near the interface, is accompanied by enhanced absorption [98]. While enhanced coupling in structured targets is typically attributed to enhanced Brunel heating, resonant excitation of plasma waves or field enhancement via Mie resonance, we find that the aforementioned quasi-static magnetic fields near the interface at supra-critical densities now play an important role in accelerating electrons to relativistic energies.

Depending on the scale of the surface perturbations (both $H$ and $\Lambda$ ), enhanced coupling can result in heated plasma blow-off that cannot be overcome by the laser profile steepening under the conditions treated. The ensuing under-dense plasma shelf that develops results

in a hot-tail that dominates the accelerated electron energy spectrum above $U_{p}^{S W}$, which is not characteristic of the high-contrast standing-wave acceleration mechanism. This tail can contribute a significant fraction of the total conversion efficiency.

Together, enhanced coupling and a structured surface drive deviations from the clean standing-wave field structure. Finally, we show that the wave-front perturbations are largely responsible for the more stochastic electron heating and randomized relativistic electron trajectories rather than quasi-static fields near the interface.

\subsubsection{Enhanced coupling from Weibel-like fields}

In the laboratory, initial target surfaces will never be free of surface perturbations like the highly idealized case previously discussed. Indeed, even targets polished to a mirror-like finish will still have some sub-wavelength structure. Surface (b) has, by most definitions, "optically flat" surface perturbations with a $\lambda_{L} / 25 R M S$ and, yet, surprisingly we see an overall 4 fold increase in conversion efficiency over the perfectly flat interface (as reported in Table 5.1).

Figure 5.4 shows our results for surface (b) using the same organization as FIG 5.2, which 
showed results for the flat surface with the temporal evolution of the (i) electron density, (ii) magnetic fields and (iii) electron angular distributions. By the peak of the pulse, the relativistic critical surface has become smoother than the initial target surface, reducing the coupling enhancing surface perturbations. While the vacuum field structure near the interface is still largely standing-wave, the perturbations in the relativistic critical surface drive transverse spatial modulations in the structure. We also note the earlier development of Weibel-like fields, likely due to the increased current densities from increased coupling.

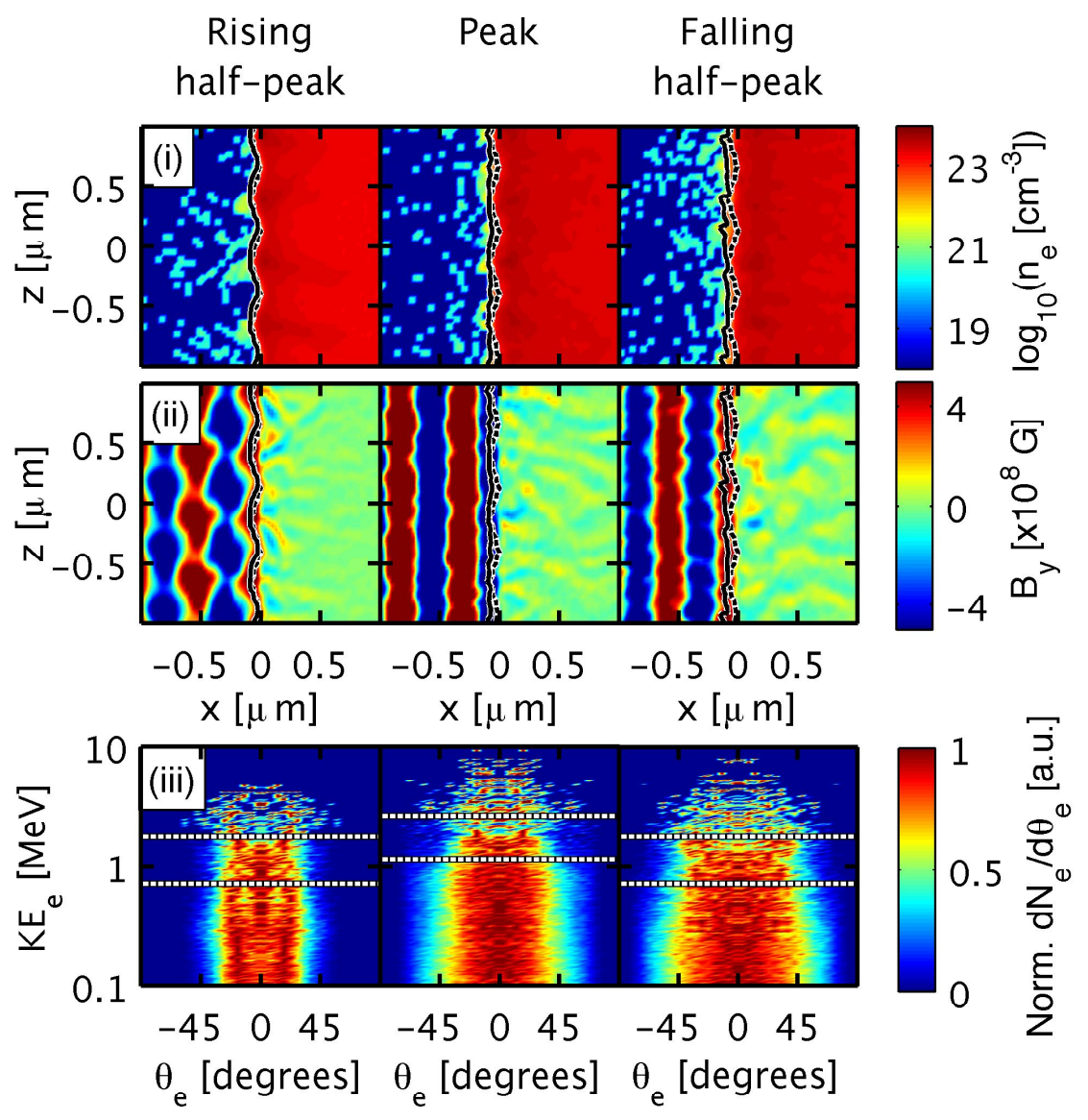

Figure 5.4: (Color) Same as Figure 5.2 but for surface (b).

Angular distributions are shown in Figure 5.4(iii) and, even with this modest surface perturbation, the bifurcation signature characteristic of the standing-wave acceleration is no 
longer present. Since Weibel-like fields played such a small role in the perfectly flat interface example, it is unlikely that this blurring is solely due to quasi-static fields generated at supra-critical densities. Since the magnitude and extent of these fields can reach $\sim 200 M G$ extended over $\sim \lambda_{L} / 4$, only electrons with kinetic energy $\lesssim 100 \mathrm{keV}$ are able to be trapped.

Figure 5.5(i) shows typical electron trajectories for the (ia) perfectly flat interface and (ib) the structure, but optically flat, interface born near the peak of the pulse when the Weibel-like fields have developed to $200 M G$ levels. The original solid density interface is indicated by the black curve and instantaneous electron kinetic energy is indicated by the $\log _{10}$ scale color bar. At supra-critical densities, low energy electron trajectories are strongly influenced by these magnetic fields. Indeed, these electrons can be redirected back into vacuum where the enhanced Brunel absorption from surface perturbations further increases this probability. Due to the alternating competition between forward direct laser acceleration in vacuum and redirecting magnetic fields inside solid density, electrons tend to 'surf' around the solid density interface until they gain enough energy from the laser to escape the magnetic field trapping and propagate deep into solid density.

This surfing provides low energy electrons multiple opportunities to reach the vacuum laser fields and is evident from the total distance electrons travel in vacuum. This integrated vacuum travel distance (normalized to $\lambda_{L}$ ) is shown in Figure 5.5(ii) where each point represents an individual trajectory of a hot-electron that eventually makes it to the diagnostic plane inside solid density. Clearly, when surface perturbations are present, we observe more electrons spending more time in under-dense regions, often over multiple excursions, increasing their probability of getting a relativistic kick from the laser. Unlike Brunel and $\vec{j} \times \vec{B}$ heating, Weibel-like quasi-static fields can improve coupling by providing low energy electrons multiple opportunities to gain energy from the laser.

These recirculated electrons only travel $\lesssim \lambda_{L} / 4$ into vacuum before they get turned around by the laser. Consistent with the majority of their energy coming from single suboptical-cycle accelerations from standing-wave-like field structures, coupling into sub- $U_{p}^{S W}$ electrons is enhanced as the number of low energy electrons that can reach the stronger vacuum laser fields is increased. From the time-integrated electron energy spectrums shown 

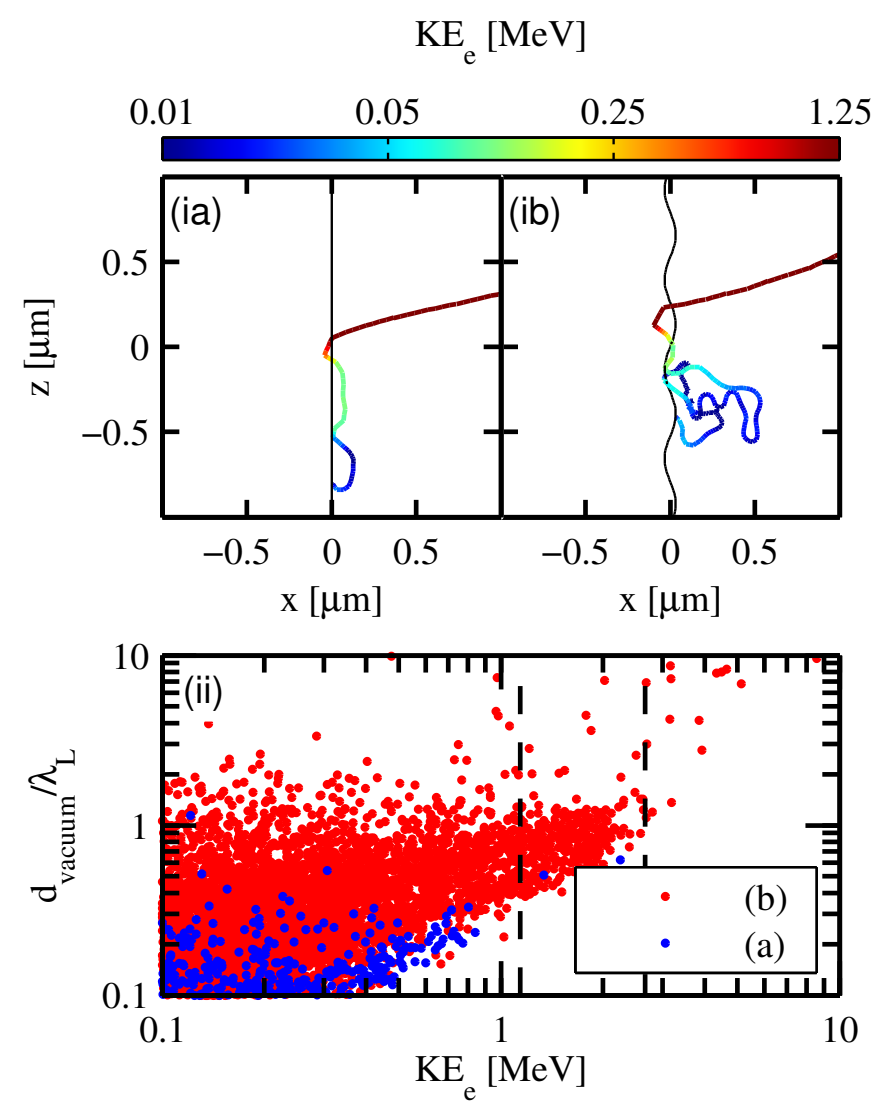

Figure 5.5: (Color) Typical hot electron trajectories, colored by instantaneous kinetic energy, from the (ia) perfectly flat interface, (ib) optically flat interface and (ii) total travel distances in vacuum as a function of maximum kinetic energy gained. The vertical dashed black lines indicate $U_{p}^{T W}$ (left) and $U_{p}^{S W}$ (right).

in Figure 5.6(i), we observe greater than 10 fold increase in $U_{p}^{T W}$ electrons (where $U_{p}^{T W}$ and $U_{p}^{S W}$ are indicated by the vertical dashed black lines).

While $96 \%$ of the total conversion efficiency from surface (b) is into electrons with energy below $U_{p}^{S W}$, we also observe the development of a hot-tail that dominates the hotelectron energy spectrum above $U_{p}^{S W}$. Similar to previous studies [33, 102], we also see two components in the electron energy distributions for these sinusoidal surface features: (1) below $U_{p}^{S W}$ dominated by sharp interface standing-wave accelerations and (2) above $U_{p}^{S W}$ dominated by under-dense accelerations. 


\subsubsection{Under-dense acceleration}

Energy distributions of electrons above $U_{p}^{S W}$ are largely Wilks-like [34] having Boltzmann distributions with slope temperatures approximately equal to $U_{p}^{T W}$ (plotted in Figure 5.6(i) as the solid black line with arbitrary normalization). The development of this quiver energy scaling part of the energy spectrum is consistent with previous experiment and simulation work $[58,66]$ with similar under-dense plasma environments with which the pulse interacted with before being reflected.

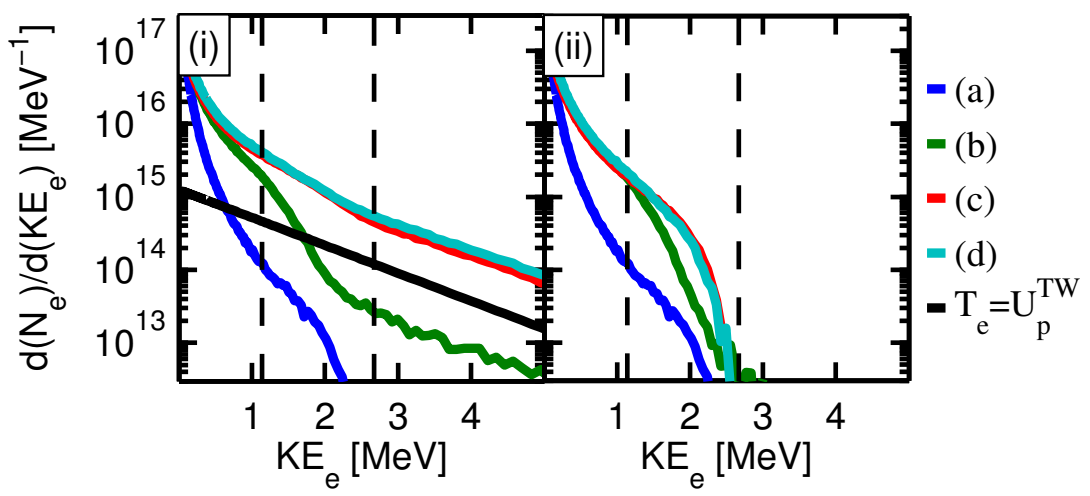

Figure 5.6: (Color) (i) Energy distribution of electrons that pass $5 \mu \mathrm{m}$ plane inside solid density on a log-linear scale for surfaces (a) through (d). The solid black line is a Boltzmann distribution with temperature equal to $U_{p}^{T W}$ (arbitrary normalization). (ii) Same distributions but with single temperature Boltzmann fit to hot-tail subtracted. The vertical dashed black lines indicate $U_{p}^{T W}$ (left) and $U_{p}^{S W}$ (right).

Using a single temperature exponential fit to electrons with energy above $U_{p}^{S W}$, we find that the hot-tail in surface $(b)$ constitutes little of the total hot-electron energy $(\sim 4 \%)$. The total conversion efficiencies for surfaces (c) and (d) were more than twice that of the optically flat surface (b) and the hot-tail electrons constitute as much as $50 \%$ of the total hot-electron energy. We can subtract out this exponential fit to the hot-tail to obtain a crude estimate for the conversion efficiency of laser energy into hot-electrons generated 
Table 5.2: Estimated conversion efficiency contributions from sharp interface standing-wave accelerations (SW) and under-dense traveling wave accelerations (TW).

\begin{tabular}{|c|c|c|c|c|}
\hline \multirow{2}{*}{ Surface } & \multicolumn{4}{|c|}{ "Conversion Efficiency, $\eta_{L \rightarrow e^{-}}[\%]$} \\
\hline & Total & $=$ Estimated SW & + & Estimated TW \\
\hline (a) & 4.6 & 4.6 & & 0 \\
\hline (b) & 21 & 20 & & 1 \\
\hline (c) & 41 & 28 & & 13 \\
\hline (d) & 47 & 23 & & 24 \\
\hline
\end{tabular}

from only the sharp interface interaction. The fractional energy contribution from these two populations is summarized in Table 5.2. The resulting distributions after subtraction are shown in Figure 5.6(ii). The predominant differences in the unmodified electron energy spectra in Figure 5.6(i) between surfaces (b) through (d) is the amplitude of the hot-tail contribution. Clearly in these high-contrast interactions, much of the enhanced absorption can come from this developing under-dense plasma and not solely from sharp interface mechanisms.

Our simulation results for surface (c) are shown in Figure 5.7. The competition between heated plasma expansion and laser profile steepening drives the relativistic critical surface to evolve throughout the interaction (Figure 5.7(i)). Additionally, we see more significant under-dense plasma shelf formation [33] through which the laser must propagate before it reflects off the relativistic critical surface. This is likely due to laser diffraction effects inside the cavities (behaving as sub-wavelength apertures) preventing laser profile steepening from overcoming heated plasma expansion. Since the aspect ratio of the cavities $(H / \Lambda \gtrsim 1)$ is of order unity, the light has to undergo multiple reflections before it can escape. These effects, coupled with enhanced absorption effectively reduces the reflectivity from within the cavities. Using an analytic technique to propagate the reflected pulse measurement at the boundary of the simulation back to the original laser focal plane, we find that the reflectivity from within the cavities was only $\sim 5 \%$ while the reflectivity from the tips was still higher than 50\%. This spatial non-uniformity in reflectivity creates regions of field 
structure more closely resembling the traveling-wave of the incident laser and, at early times, is the source of the Wilks-like hot-tail in the electron energy distribution.

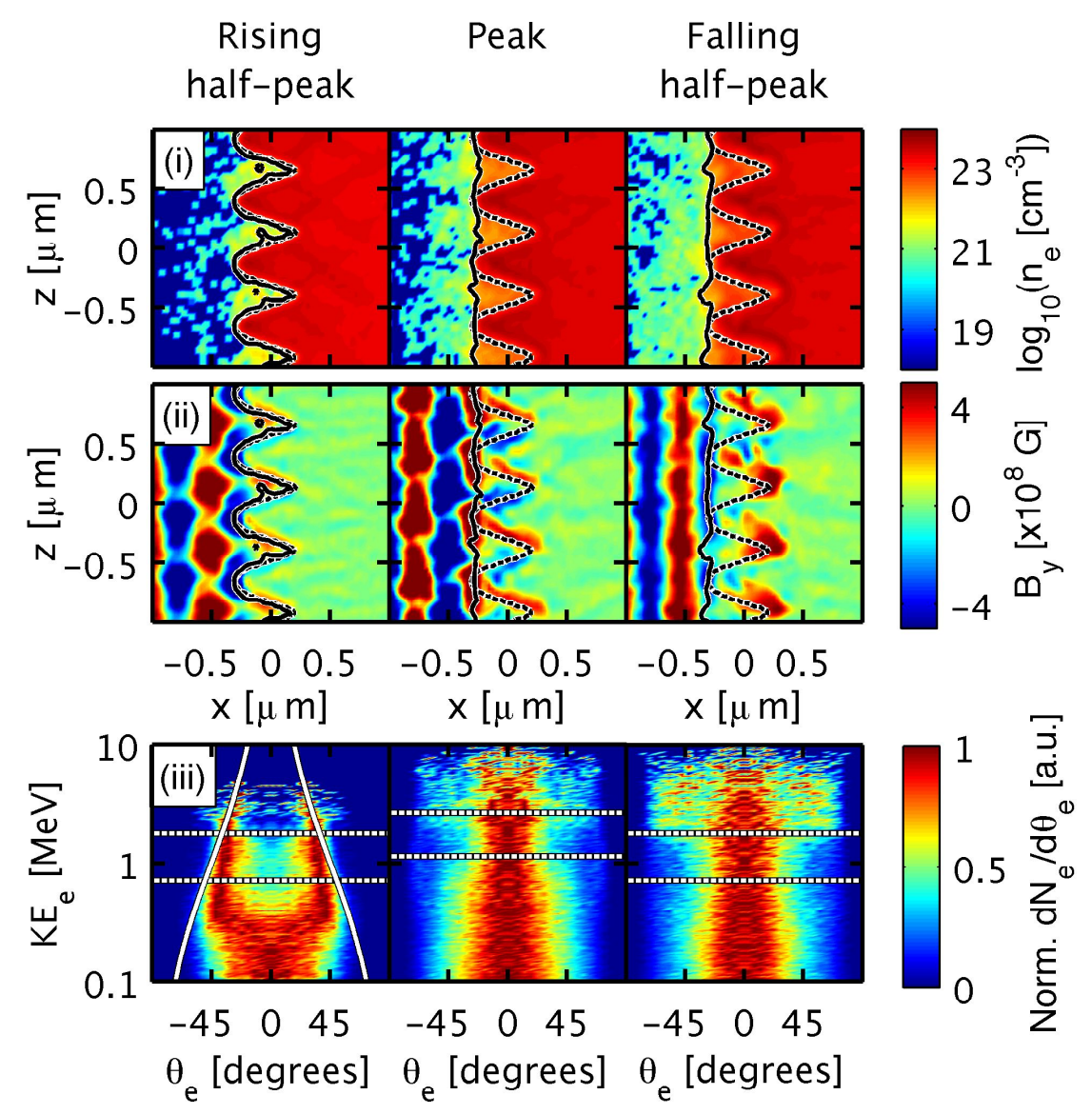

Figure 5.7: (Color) Same as Figure 5.2 but for surface (c). The solid white curves in (iii) indicate the classical ejection angle.

At early times before the cavities can fill with supra-critical density plasma, there is again a clearly bifurcated angular distribution for electron energies above $>U_{p}^{S W}$ (Figure 5.7(iii) for the rising half-peak intensity). Electron motion in an ideal traveling-wave (i.e. planewave) can be characterized with a simple energy-angle relationship given by the classical ejection angle $\theta_{c}$ (for electrons that start from rest) as

$$
\tan \left(\theta_{c}\right)=\frac{p_{\perp}}{p_{\|}}= \pm \sqrt{\frac{2}{\gamma-1}}
$$


where $p_{\perp}\left(p_{\|}\right)$is the perpendicular(parallel) component of the momentum vector with respect to the laser propagation axis. This solution is plotted on top of the electron distributions for as a solid white curves in Figure 5.7(iii). Consistent with previous assessments, electrons with energy above $U_{p}^{S W}$ are clearly from traveling-wave acceleration in under-dense plasma and not from sharp interface mechanisms. The possible phase space that accelerated electrons can fill by this traveling-wave (at half-peak intensity) are shown in Figure 5.8. Unlike those distributions shown in Figure 5.3, this calculation does not incorporate the injection or dephasing of electrons as their initial positions and momenta are unknown due to the underdense plasma expansion. However, from this simple calculation we find that electrons with initial kinetic energy less than $100 \mathrm{keV}$ can only be accelerated by this plane wave up to approximately $9 \mathrm{MeV}$, consistent with the maximum laser energy observed in the simulation. Also, unsurprisingly, electrons injected into the field with momenta along the laser propagation axis are more forward oriented (inside the dark blue classical ejection angle curve) than those injected with momenta opposite the laser propagation axis (outside the dark blue classical ejection angle curve).

Once the cavity fills with supra-critical density plasma, the clear bifurcation disappears. At this time, gradient driven magnetic fields begin to develop in the now over-dense cavities resulting in $\sim 500 M G$ fields extending over $\lambda_{L} / 2$ by the end of the interaction. These fields can trap even relativistic electrons $(\lesssim 1 \mathrm{MeV})$. Despite having these quasi-static magnetic fields at the interface that are larger and stronger than any Weibel-like fields, the angular distribution of electrons narrows as the critical surface smooths over the solid density surface features. While most certainly being capable of strongly perturbing even relativistic electron trajectories, quasi-static fields near the solid density interface (yet again) cannot be predominantly responsible for the shape of these electron angular distributions.

\subsubsection{Quasi-static fields vs. wave-front perturbations}

For surface (d) (Figure 5.9), the laser is able to propagate inside the cavities at all times more-or-less preserving the original surface perturbation morphology. Like in surface (c), we have increased under-dense plasma shelf formation and spatial non-uniformity in specular 


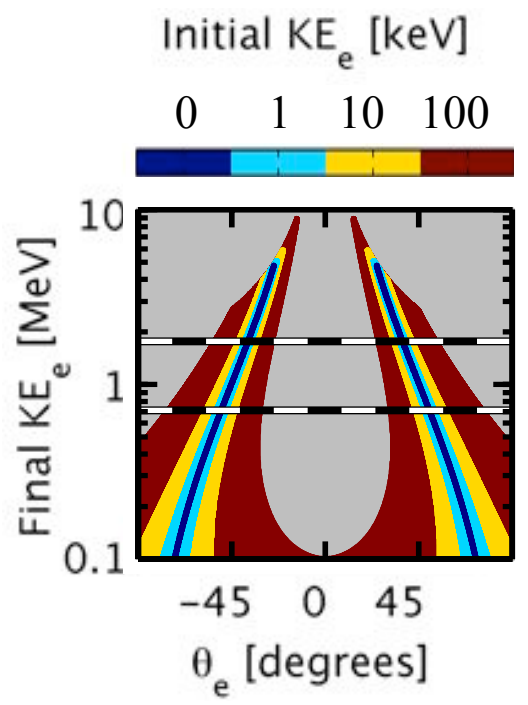

Figure 5.8: (Color) Hot-electron phase space calculations of test particles accelerated by traveling-wave EM fields as a function of final kinetic energy and angle (colored by initial kinetic energy). The horizontal dashed white lines indicate $U_{p}^{T W}$ (lower) and $U_{p}^{S W}$ (upper).

reflectivity yielding the same angular bifurcation effect dominating electrons with energy greater than $U_{p}^{S W}$ at early times as seen from Figure 5.9(iii). While the bifurcation has again filled by the peak of the pulse, we now find that the angular distribution remains broad at all energies throughout the entire interaction. Again, this is clearly not just due to the Weibel-like fields inside solid density as this distribution is significantly broader at higher energies than surfaces (a) through (c) where these fields are just as strong (or stronger).

Rather, the difference appears to be the spatial scales over which the laser wavefronts are perturbed. For surface (c), the perturbations in the wave-fronts gradually flatten with the smoothing of the relativistic critical surface. For surface (d), however, we can clearly see continually growing perturbations in the wavefronts on sub-wavelength (and sub-trajectory) scales resulting in the increasingly more stochastic heating and randomization of electron trajectories. 


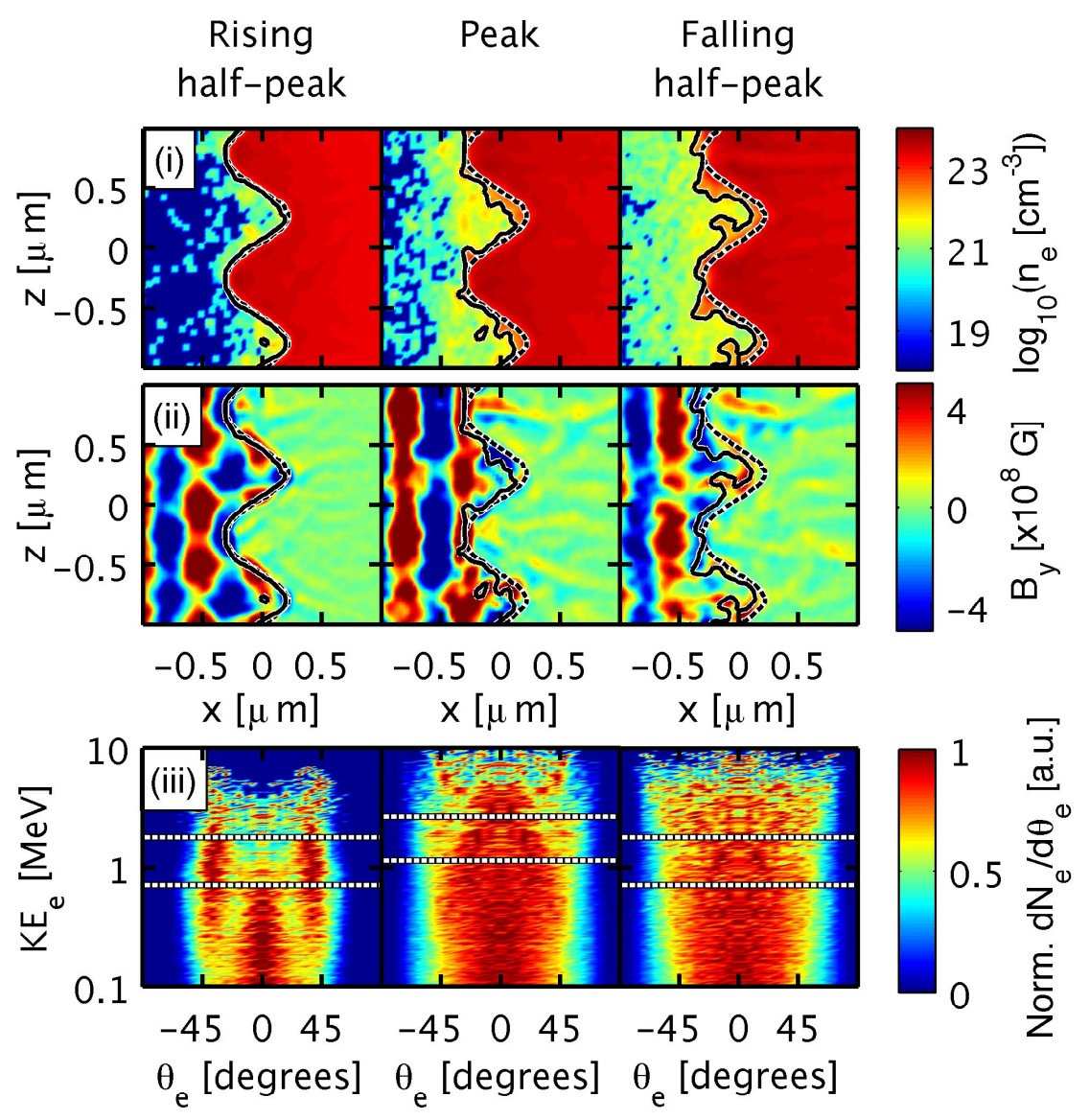

Figure 5.9: (Color) Same as Figure 5.2 but for surface (d).

\subsection{Summary}

In conclusion, we have shown that electrons accelerated from sharp, perfectly flat interfaces are characteristic of the standing-wave acceleration mechanism where the skin depth effect is essential for injecting low energy electrons into the laser in initially cold targets. In addition to previous coupling mechanisms used to describe enhanced absorption at sharp density interfaces, we find that developing Weibel-like quasi-static magnetic fields near the interface at supra-critical densities can also enhance absorption by trapping and re-injecting low energy electrons from beyond a skin depth inside solid density back into the laser fields for multiple acceleration opportunities. Conversion efficiencies in these simulations above $25 \%$ are attributed to under-dense accelerations and not from the sharp interface standing- 
wave acceleration mechanism. While it has been speculated that quasi-static magnetic fields near the interface at supra-critical densities could affect relativistic electron transport, we find that it plays only a minor role compared to wave-front perturbations and accelerations in stochastic fields.

We find this pulse/target combination to be in a transient regime where initial target conditions can significantly influence early absorption physics but can still evolve quite drastically throughout the interaction. For significantly shorter pulses, it is likely that the interface will not significantly change throughout the interaction and under-dense plasma expansion will be minimal. Therefore, accelerated electrons should be very sensitive to the initial surface roughness and be typical of the early (rising edge) pulse behavior of these $100 \mathrm{fs}$ pulses. For surfaces (a) and (b), this means coupling would likely be dominated by the standing-wave acceleration mechanism with coupling predominantly into electrons with energy below $U_{p}^{S W}$. Since the widths of these angular distributions are sensitive to the target temperature and electron density (Figure 5.3), we would surmise that the angular distributions would be narrower for the shorter pulse (with the same peak intensity) since the interaction interface is not likely to heat as much as or be at a lower density than the $100 \mathrm{fs}$ pulse cases. For surfaces (c) and (d), it's likely that the angular distributions will look very similar to the rising edge of the $100 \mathrm{fs}$ pulse cases throughout the interaction, where electrons with energy above $U_{p}^{S W}$ will be accelerated into the target at the classical ejection angle. For significantly longer pulses, none of these sharp interface, absorption enhancing features are likely to survive. However, under-dense plasma expansion and quasisteady-state critical surface perturbations [148] can provide additional coupling mechanisms into electrons with energy well above $U_{p}^{S W}$ that should eventually dominate the conversion efficiency over the early sharp interface phenomenon. Certainly, another pulse/target pair could produce radically different conditions throughout the interaction and as such merits further study.

We would like to acknowledge useful conversions with R. Fedosejevs, F. Fiuza, A. J. Kemp, R. B. Stephens and S. C. Wilks. This work was performed under DOE contract DEAC52-07NA27344 with support from the Lawrence Scholar Program, OFES-NNSA Joint 
Program in High-Energy-Density Laboratory Plasmas and an allocation of computing time from the LLNL Grand Challenge. 


\section{Chapter 6 \\ SPECULAR REFLECTIVITY AND ABSORPTION IN HIGH-CONTRAST RELATIVISTIC LASER-PLASMA INTERACTIONS}

In the previous chapter, I showed how high-contrast pulses can convert energy into relativistic electrons near a sharp supra-critical interface, free of pre-plasma, using shorter $100 \mathrm{fs}$ pulses and highly simplified target surface perturbations. In reality, like for the experiment discussed in Chapter 2, laser pulses can be significantly longer ( $\gtrsim 5 \times$, Figure 2.7) and commercially available targets can have wavelength scale perturbations with complicated, multi-modal spectral intensity distributions (Figure 2.10). Both of these factors play a role in determining the evolution of the laser-plasma interface throughout the interaction and ensuing accelerated electron properties. Since a variety of surface features were (albeit unintentionally) shot during the experiment, a selection of typical surfaces were chosen to study their effect on hot-electron generation, discussed in Chapter 6.3 using two pulse durations: a shorter $100 \mathrm{fs}$ pulse (identical to that chosen in Chapter 5 for simplicity and computational convenience) and a longer $500 \mathrm{fs}$ pulse characteristic of the experiment.

Direct measurements of the hot-electrons generated at the interaction interface are not directly observable in experiment, but many indirect approaches have been proposed that rely on electron transport through the target bulk [25, 27, 64, 65, 70, 71, 74]. Although often neglected, measurements of the specularly reflected pulse have also been shown to provide 
invaluable insight into the laser-plasma interaction [35, 42, 78, 82, 149]. While still not a direct measurement of the desired hot-electrons, specular reflectivity measurements provide a direct measurement of the laser-plasma interaction and can constrain simulations (discussed in Chapter 6.1) used to infer hot-electron properties characteristic of the experiment discussed in Chapter 2.

All the interesting physics that affects the specular pulse properties, such as absorption, relativistic non-linearities, hole-boring, field ionization etc, occurs near the interaction interface and many of these phenomena are nonlinearly dependent on intensity and electron density profiles. Since the pulse has a finite focal spot (Figure 2.8), we can expect there to be some spatial dependence on these effects in the near-field. However, all experimental measurements are made in the far-field, after diffraction effects have fully manifested. Therefore, the spatial distributions of the specularly reflected pulse in the near-field may not be indicative of those in the far-field where all experimental measurements are taken. A priori, it is not quantitatively obvious how significant this redistribution of laser energy will affect the interpretation of experimentally obtained far-field measurements.

Characterization of the specularly reflected pulse near the interaction with simulations is complicated by the presence of the supra-critical plasma target by electrostatic fields, quasistatic magnetic fields, noise from the kinetic nature of the macro-particles and, of course, the interference between the incident and reflected pulses. Instead, the specular field is recorded at the inlet/outlet boundary of the simulation (20 $\mu \mathrm{m}$ away from the target interface) where these influences are negligible. However, even a few tens of microns away, diffraction effects have drastically reshaped the specular pulse from the desired measurement near the interaction interface. Also, propagating the light to the far-field with these same simulations would be prohibitively expensive since we would have to resolve the light propagation over millimeters or even centimeters of space before the diffraction pattern would converge. Alternatively, both the near and far-field properties of the specularly reflected pulse are obtained by analytically propagating the boundary measurements first backwards, toward the original laser focal plane $(x=0 \mu m)$, to obtain the near-field distribution and then forwards to the far-field centimeters away. Multiple techniques exist and and have been 
discussed previously; here I use the non-paraxial approach discussed in Chapter 4.2 .2 to handle sub-wavelength spatial modes in the specularly reflected pulse.

Finally a summary of what can be learned from specular reflectivity measurements is made in Chapter 6.4.

\subsection{PIC simulations}

\subsubsection{Setup}

$2 D 3 V$ Cartesian fully kinetic particle-in-cell simulations were performed to determine the effect of realistic surface perturbations on specular reflectivity measurements and hot-electron generation using the commercially available PIC code LsP [104]. The setup, Figure 6.1, is similar to those discussed in Chapter 5 with the exception that now we use multi-modal, realistic target surfaces instead of the single mode sinusoidal features. The target was mod-

eled as a solid density $\left(n_{e}=1.81 \times 10^{23} \mathrm{~cm}^{-3}\right)$, initially $Z=3$ charge state Aluminum slab, $75 \mu m$ deep by $150 \mu m$ wide with $25 \mu \mathrm{m}$ of vacuum in front of the target to accommodate electron refluxing (with absorbing boundary conditions). Both incident and specularly reflected pulse field quantities were recorded at the inlet boundary of the simulation $(x=-20 \mu m)$. Hot-electron properties (time, energy and angle) are recorded as they pass through a diagnostic plane at $x=5 \mu \mathrm{m}$ inside solid density where $x=0 \mu \mathrm{m}$ corresponds to the (mean) solid density interface and incident laser focal plane (in vacuum).

The variety of surface features observed in the experiment are illustrated in Figure 6.2. On the left are the same surface spectral intensities from Figure 2.10 from the AFM measurements for (a) the low resolution, wide area scan of spot size or 'large' features and (b) the high resolution, narrow area scan of wavelength scale or 'small' features. The range of the spectral power distributions are shown as the shaded gray area. Three unique spectral distributions were observed (indicated by the solid and dashed black lines) with RMS values ranging between 100 and $400 \mathrm{~nm}$. Four surfaces were reconstructed with representative spectral distributions and RMS as the data, shown in subplots (ai)-(aiv) and 
LASER:

Polarized in the plane Normally incident

$\lambda_{\mathrm{L}}=527 \mathrm{~nm}$

$\tau_{\mathrm{FWHM}}=100-500 \mathrm{fs}, \sin ^{2}$

$\mathrm{Z}_{\mathrm{FWHM}}=8 \mu \mathrm{m}$ Gaussian (f/40)

$\mathrm{I}_{\text {peak }}=4.65 \times 10^{19} \mathrm{~W} / \mathrm{cm}^{2}$

\section{SIMULATION:}

LSP, direct-implicit

Absorbing boundaries

Electrons: 144-196 per cell

Ions: 25-49 per cell

Resolution: $\lambda_{\mathrm{L}} / 16, \mathrm{~T}_{\mathrm{L}} / 64$
$100 \mu \mathrm{m}$

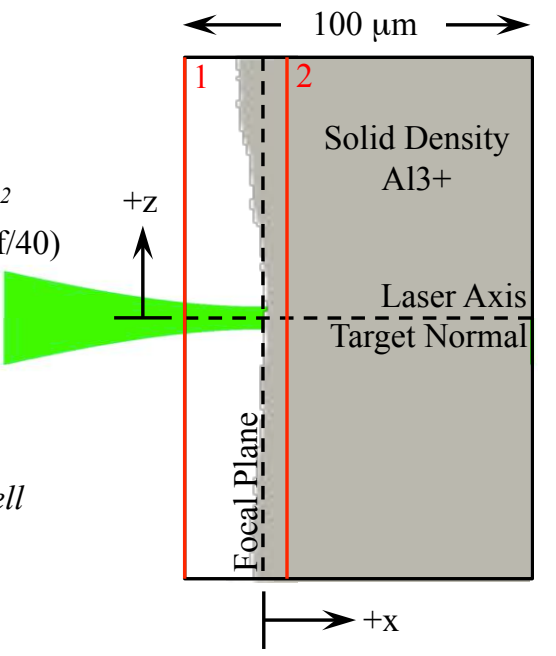

\section{PLASMA TARGET:}

Initially Al3+

Solid density $\left(\mathrm{n}_{\mathrm{e}}=1.81 \times 10^{21} \mathrm{~cm}^{-3}\right)$

Sequential field ionization (ADK)

Fully kinetic electrons and ions

Collisionless

Initially $\mathrm{T}_{\mathrm{e}}=\mathrm{T}_{\mathrm{i}}=5 \mathrm{eV}$

DIAGNOSTIC PLANES:

1. Laser fields $(\mathrm{x}=-20 \mu \mathrm{m})$

2. Hot-electrons $(\mathrm{x}=5 \mu \mathrm{m})$

Figure 6.1: (Color) High-contrast specular reflectivity and absorption simulation setup.

indicated by color. Subplot (ai) is characteristic of the Goodfellow foils (Figure 2.10(a)) and (aii)-(aiv) are characteristic of both horizontal and vertical perturbations in the grating like electroplated targets (Figure 2.10(b)). Of the small features, a single distribution was observed across all targets at the sub-wavelength scale (indicated by magenta) with a $25 \mathrm{~nm}$ RMS.

The incident laser pulse $\left(\lambda_{L}=527 \mathrm{~nm}\right.$, period $T_{L}=1.76 \mathrm{fs}$, corresponding to a nonrelativistic critical density $\left.n_{c}=m_{e} \omega_{L}^{2} /\left(4 \pi e^{2}\right)=4.0 \times 10^{21} \mathrm{~cm}^{-3}\right)$ has a $200 \mathrm{fs}(1 \mathrm{ps})$ duration $\sin ^{2}$ intensity profile with $100 \mathrm{fs}(500 \mathrm{fs})$ FWHM incident normally on the target with an $8 \mu \mathrm{m}$ focal spot FWHM (approximately Gaussian with an $f / 40, e^{-2}$ full angle divergence), polarized in the simulation plane, with a cycle averaged peak intensity of $4.65 \times 10^{19} \mathrm{~W} / \mathrm{cm}^{2}$. The quiver energy $U_{p}$ of electrons in the oscillating electric field is given by $U_{p}^{T W}=\left(\gamma_{o}^{T W}-1\right) m_{e} c^{2}=1.1 \mathrm{MeV}$ where $\gamma_{o}^{T W}=\sqrt{1+a_{o}^{2}}=3.2$ is the relativistic Lorentz factor and $a_{o}=e E_{o} / m_{e} \omega_{L} c=3.1$ is the normalized vector potential (where the $T W$ superscript refers to the vacuum value traveling-wave field structure).

The simulation was performed using a direct implicit algorithm incorporating an energyconserving particle push [104]. The grid had a spatial resolution of $31.2 \mathrm{~nm}\left(\sim \lambda_{L} / 16\right)$ 
(a) LARGE features

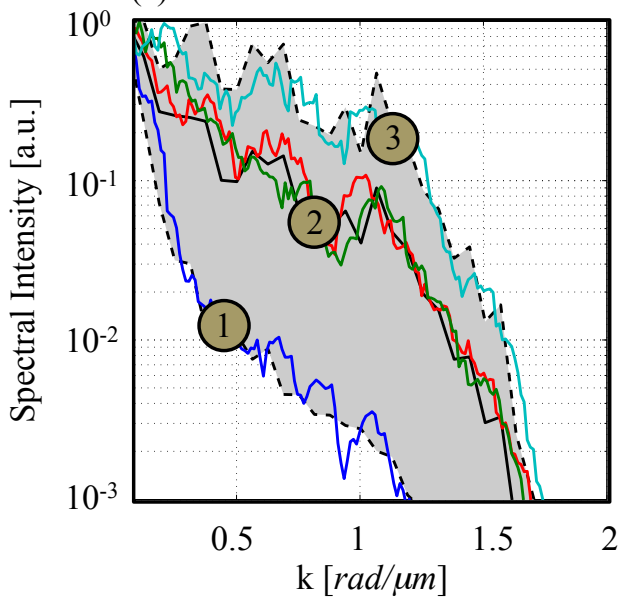

(b) SMALL features

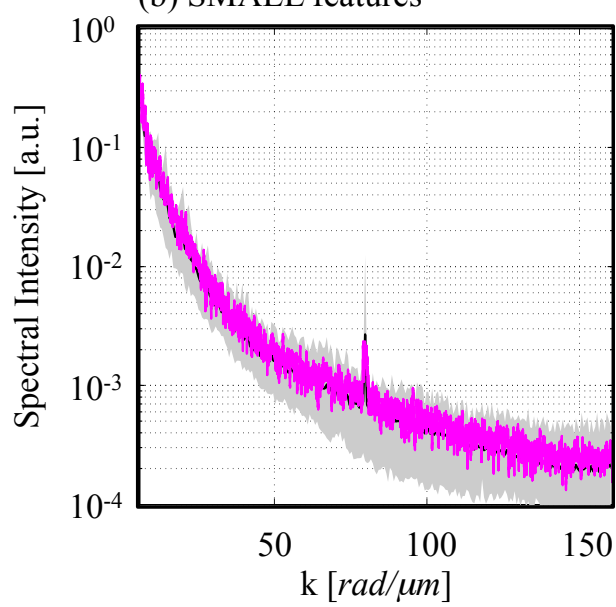

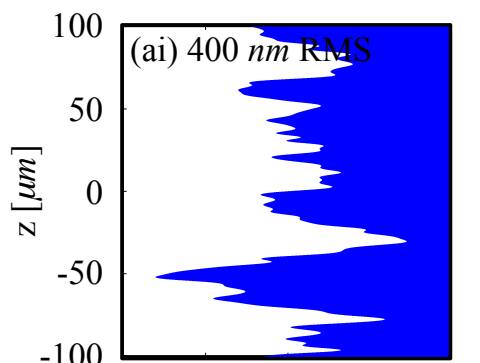
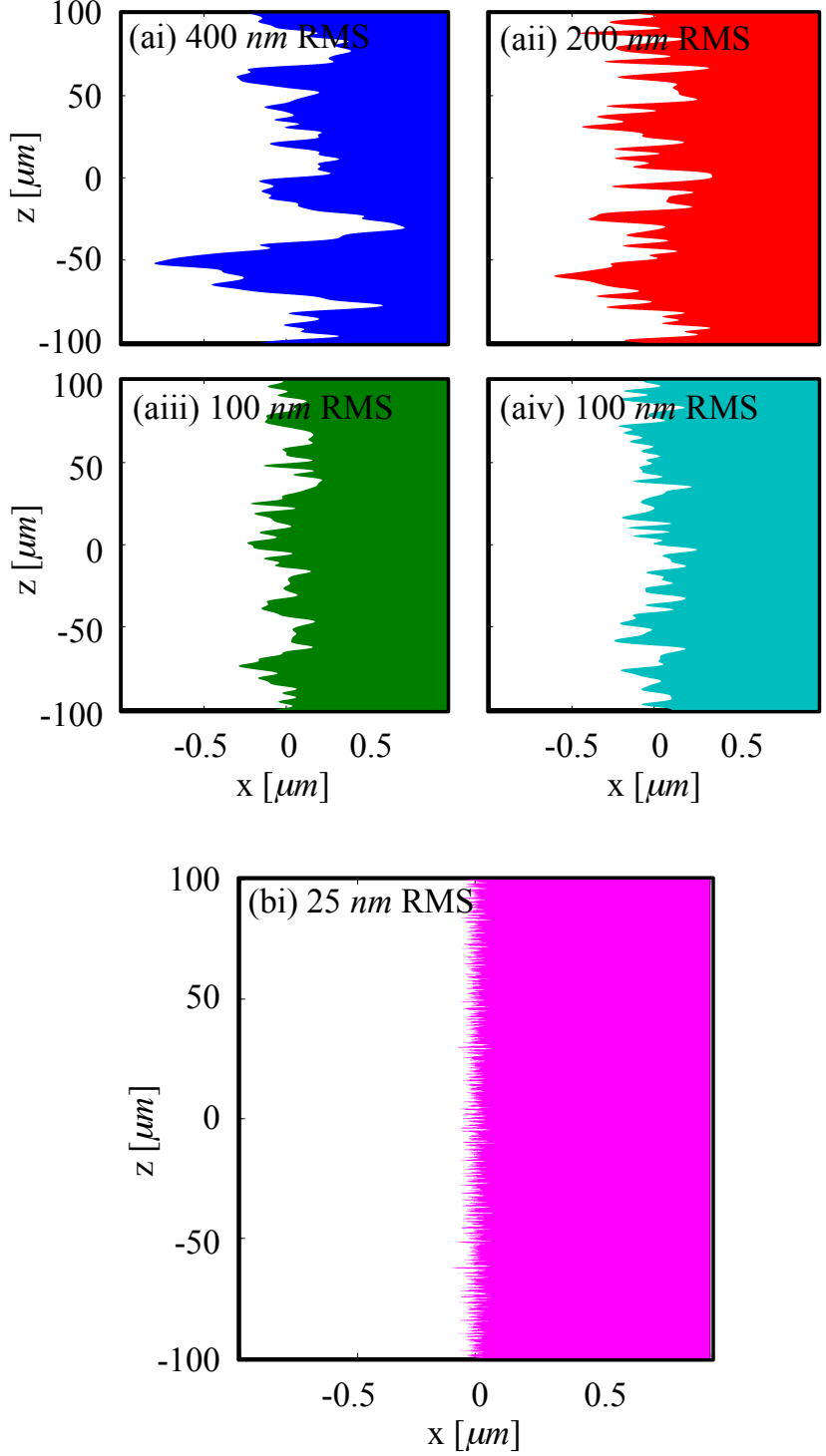

Figure 6.2: (Color) (a) Range of surface feature spectral intensity distributions from the experiment (shown as shaded gray area) with the four unique large scale features (indicated by the different colors) from the supra-wavelength resolution scan and (b) the single unique surface from the sub-wavelength resolution scan.

and temporal resolution of 27.5 as $\left(\sim T_{L} / 64\right)$. Through a separate study, hot-electron distributions in time, space, energy and angle were found to be sufficiently converged at these resolutions. Electron macro-particle densities ranged from 144 to 196 per cell and the ions ranged from 25 to 49 per cell resulting in $\sim 625 M$ macro-particles in the simulation. 
All electron and ion species were fully kinetic and collisionless with initial electron and ion temperatures of $5 \mathrm{eV}$. Sequential tunneling field ionization was included using ADK (Ammosov, Delone and Krainov) ionization rates for ions in an alternating electromagnetic field [87].

\subsubsection{Interaction region evolution}

Since a variety of surface feature were shot during the experiment, we begin the discussion with a single surface roughness characteristic of the experiment: the large scale features of the red surface plus the small scale magenta features from Figure 6.2 and the $500 \mathrm{fs}$ pulse. The temporal evolution of the interaction is outlined in Figure 6.3 at four instances in time, indicated by the different rows: (a) initial conditions, (b) rising half-peak upon reflection, (c) peak upon reflection and (d) trailing half-peak upon reflection. The first column (indicated by $i$ ) is the electron density profile, normalized to critical density, shown on a $\log _{10}$ scale. The second column (indicated by $i i$ ) is the cell averaged ionization state. The third column (indicated by iii) is the near-field cycle averaged laser intensity also shown on a $\log _{10}$ scale. The complex structure in the near-field intensity pattern is due to the interference between the smooth incident pulse and the structured specularly reflected pulse due to the surface perturbations and high reflectivity of the target. In all these figures, the initial and current relativistic critical surfaces are indicated by the gray and black curves respectively (calculated for each z-position as where the $\gamma n_{c}$ curve intersected $n_{e}$, where $\gamma=\sqrt{1+a_{o}^{2} / 2}$ was calculated using the local field intensity). From these figures, we can see that as the interface becomes increasingly ionized, the relativistic critical surface expands out into vacuum accompanied by increased under-dense plasma environments. ${ }^{36}$

\footnotetext{
${ }^{36}$ Note that there is an artificial interface in the simulation at $x=1 \mu m$, apparent in the density and charge state figures at late times, but it appeared to have little influence on the LPI or electron generation.
} 


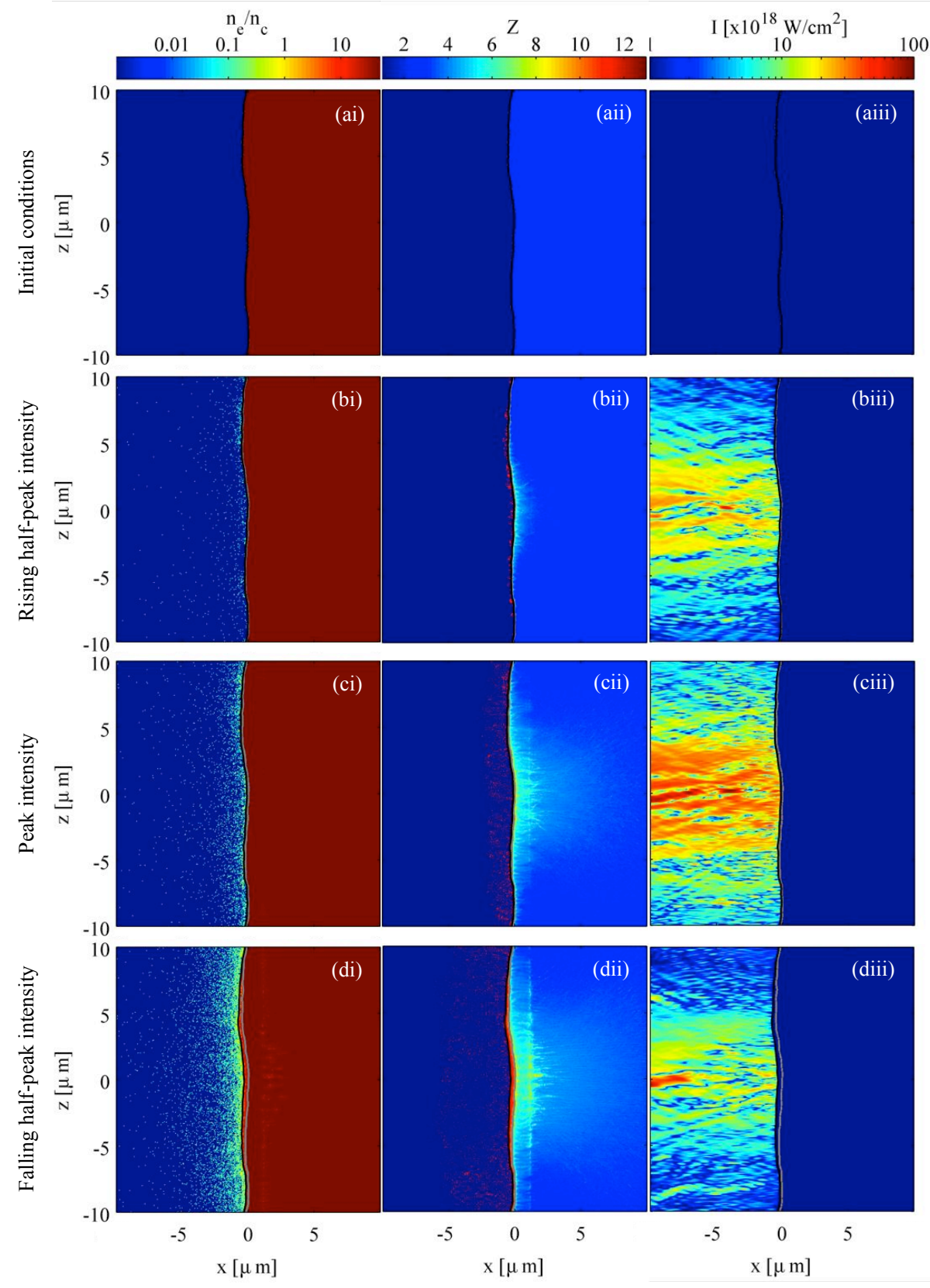

Figure 6.3: (Color) Simulated evolution of the high-contrast laser-matter interaction at various snapshots in time, indicated by the different rows: (a) initial conditions, (b) rising half-peak upon reflection, (c) peak upon reflection and (d) trailing half-peak upon reflection. The first column (indicated by $i$ ) is the electron density profile, normalized to critical density, shown on a $\log _{10}$ scale. The second column (indicated by $i i$ ) is the cell averaged ionic charge state. The third column (indicated by iii) is the near-field cycle averaged laser intensity also shown on a $\log _{10}$ scale. The initial and current relativistic critical surfaces are indicated by the gray and black curves respectively. 


\subsection{Specular reflectivity}

Unlike the previous low-contrast simulations, there is significantly less under-dense plasma present near the interaction region throughout these high-contrast simulations. Therefore, it is likely that under-dense plasma phenomena due from field ionization or relativistic selfphase modulation will be negligible. We also note that by the falling half-peak intensity of the pulse, the relativistic critical surface is no longer the same as the original surface perturbations (Figure 6.3(d)).

\subsubsection{Near-field}

Shown in Figure 6.4 are spatially and temporally resolved estimates of the near-field spectral shifts (given as a percentage of the incident pulse carrier) due to (i) Doppler shifting from motion of the relativistic critical surface, (ii) relativistic self-phase modulation and (iii) their combined effect for (a) the fixed and (b) the dynamically ionizing simulations. The Doppler shift was calculated by tracking the motion of the relativistic critical surface (with a $20 \mathrm{fs}$ resolution, indicated by the black curve in Figure 6.3). The Doppler shift upon reflection is then simply given by Equation 1.32. The relativistic self-phase modulation was estimated, following Watts [42], using Equation 1.30 and the temporally evolving under-dense plasma density from the simulation. ${ }^{37}$ In each case, the incident laser intensity profile is assumed to be unperturbed. For clarity, the 1 and $50 \%$ peak intensity contours of the incident laser are indicated by the dashed black curves and any intensity below $0.1 \%$ is truncated (shaded gray areas). As anticipated, there is virtually no early shifting from ionization or relativistic self-phase modulation from under-dense plasma propagation, but the spectrum is overall blueshifted which appears to be from ionization induced critical surface expansion resulting in Doppler shifts. On the trailing edge of the pulse, the heated plasma expansion out into vacuum overcomes the laser radiation pressure, turning into a late blueshift of about $1 \%$ from Doppler motion.

\footnotetext{
${ }^{37}$ In particular, the integral was performed up to the relativistic critical surface and no phase modulation of the backwards propagating pulse was included (which should be significantly less since the specularly reflected pulse has significantly lower intensity).
} 

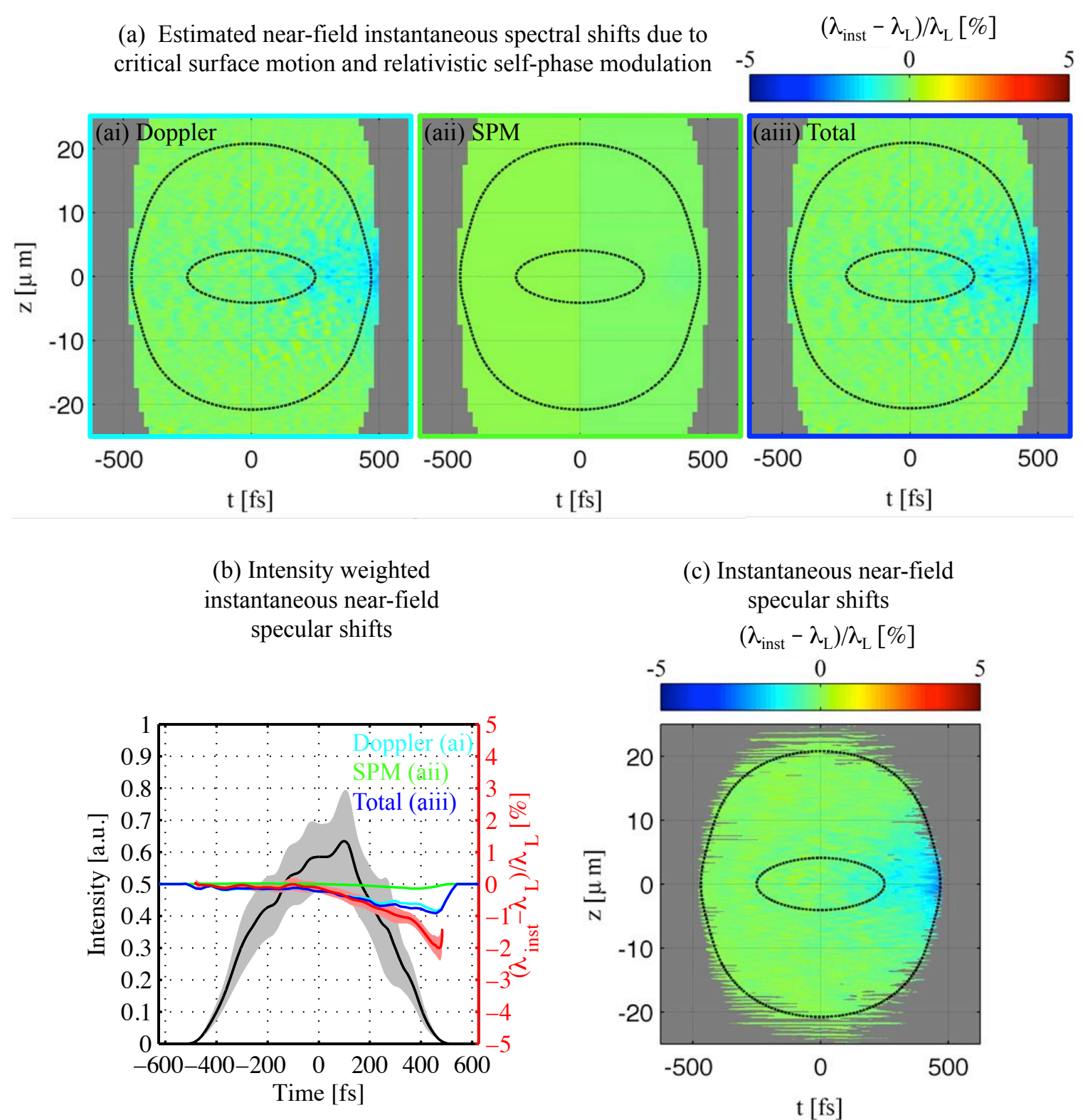

(b) Intensity weighted specular shifts (c) Instantaneous near-field specular shifts

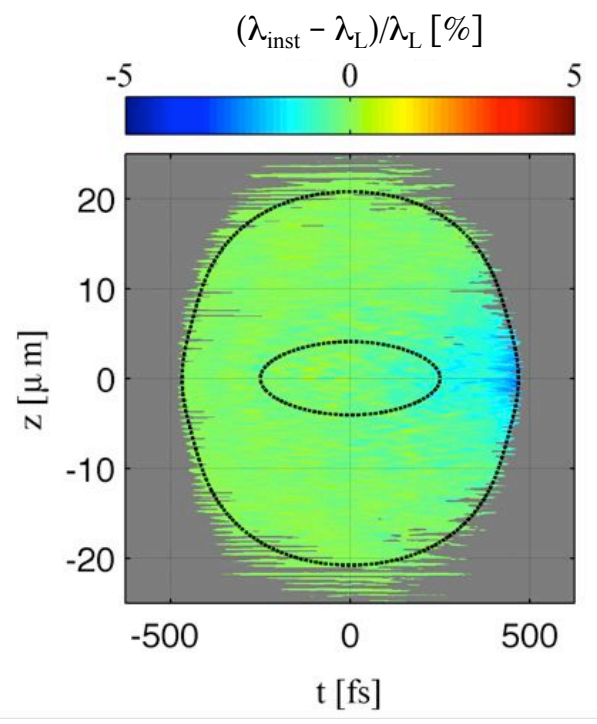

Figure 6.4: (Color) Estimated near-field spectral shifts due to (ai) Doppler shifts from the motion of the relativistic critical surface, (aii) relativistic self-phase modulation and (aiii) their combined effect. (b) Spatially integrated, intensity weighted intensity (black/gray) and instantaneous wavelength shift (red/pink) of the specularly reflected light (shaded areas indicate $\left.\pm \sigma_{S T D}\right)$. Also shown are the equivalent instantaneous wavelength shifts from (ai) the doppler shift (cyan), (aii) relativistic self-phase modulation (green) and (aiii) their combined effect (blue). (c) Instantaneous near-field specular wavelength shifts resolved in space and time. The dashed black contours indicate the 1 and $50 \%$ peak intensity contours of the incident laser pulse. Note: the color scales have been chosen for comparison with the shifting observed from the low-contrast interactions of Chapter 4. 
Since there is very little plasma development, even by the end of the interaction, we can use the non-paraxial propagation approach previously discussed to propagate the specularly reflected pulse measurement (made at the boundary of the simulation) back toward the laser interaction region. In this manner, we can directly study the near-field properties of the specularly reflected pulse before diffraction effects have convolved its interpretation, without the influence of fields present in the plasma near the interaction interface, and without interference between the incident and reflected pulses. The spatially integrated results are shown in Figure 6.4(b) with comparison to the various phenomena in (a). Similar to the low-contrast results, the estimates are linearly weighted by the intensity on target and the specular reflected pulse is weighted by the cube of the intensity (what the experimental PG FROG measurement would have seen). The spatially and temporally resolved wavelength shifts of the specularly reflected light (propagated back to the original laser focal plane) are shown in (c) and the match is quite good with the estimated shifting in (aii) up until the falling half-peak of the pulse. ${ }^{38}$ Again, we see how nicely the non-paraxial algorithm works since the propagated pulse fits nicely within the incident envelopes (the signal was again truncated at $0.1 \%$ of the peak intensity value, shaded gray for clarity).

The amount of critical surface motion on the trailing edge of the pulse will be sensitive to the heating of the target. Depending on the target composition, this blueshift could be strongly sensitive to the electron heat capacity of the target. The heat capacity of the target will depend largely on getting the charge state correct in the interaction region. These simulations include field ionization, but not collisional ionization or recombination. ${ }^{39}$ While collisional effects can play a larger role at solid density, it's believed that the LPI region rapidly heats to a nearly collisionless state $[35,102]$ where hot-electron generation is dominated by collisionless mechanisms $[90]\left(\lesssim \gamma n_{c}, \gtrsim 1 \mathrm{keV}\right)$. This also means that the heat capacity at solid density near the interface, where collisional effects aren't negligible, will be wrong and correspondingly the heated under-dense plasma expansion. The reduced heat capacity at distances beyond a laser few skin depths, where field ionization is negligible,

\footnotetext{
${ }^{38}$ At this time, it is unclear why this is the case.

${ }^{39}$ This is a work in progress for these simulations.
} 
however, was found to be adequate for these aluminum targets/pulse combinations. ${ }^{40}$

\subsubsection{Far-field}

In high-contrast interactions, the shape of the critical surface solely determines the differences in the far-field intensity distributions of the incident and specularly reflected pulses. Since all the spectral shifting that occurs throughout the interaction originates in the nearfield close to the laser-plasma interaction interface, diffraction of the beam in the far-field can produce a drastically different intensity distribution where experimental measurements are made. A priori it is not obvious how this far-field diffraction will vary the interpretation of the near-field physics. Shown in Figure 6.5 are the spatially and temporally resolved intensity (first column,normalized, $\log _{10}$ color scale) and instantaneous wavelength shift (second column) in the near-field (first row) and far-field (second row) of the specular pulse. For the far-field, the collection window of the Spectralon ${ }^{\circledR}$ used in the experiment are indicated by the horizontal black lines $(\approx 82 \%$ of the pulse energy is captured inside this window). Shown in Figure 6.6 are, once again, the (a) near and (b) far-field instantaneous properties of the pulse but this time averaged in space. Since the shape of the critical surface affects far-field diffraction, the dynamically changing critical surface throughout the interaction distort the temporal intensity profile (particularly after the peak of the pulse). In contrast, the intensity weighted instantaneous wavelength shift appears, on average, to be unaffected. However, the spread of the spectral shifting has significantly narrowed (shaded pink), indicating that the far-field pattern is dominated by the high-intensity portions of the pulse. This is great news for experimental measurements slightly off of the specular axis

\footnotetext{
${ }^{40}$ This was tested with additional simulations that used fixed ionization state $A l 3+$ and $A l$ 13+ targets, albeit using shorter $100 \mathrm{fs}$ pulses. The $\mathrm{Al} 3+$ target had fewer free electrons into which the laser could couple energy, and hence lower heat capacity (as well as less mobile ions) than the $A l 13+$ target. While the integrated spectra for all three cases were similar, subtle differences appeared in the temporal evolution of the specularly reflected pulse. For the target with field ionization, the instantaneous wavelength shift was blue at all times (Figure 6.7(c), likely due to the ionization induced blueshift), ending in a $\approx 0.5 \%$ blueshift. For the fixed ionization targets, the instantaneous wavelength didn't blueshift until after the peak of the pulse had reflected (likely due to the thermal pressure of the heated target taking over, resulting in a blue Doppler shift). On the trailing edge of the pulse, the $A l 3+$ target had $\approx 0.5 \%$ blueshift where the Al $13+$ target showed $\approx 0.75 \%$ blueshift. Since the difference in these late shifts are within the range of the (transverse) spatial variations in the measurement $(\approx \pm 0.25 \%)$, this effect is thought to be negligible under these conditions.
} 

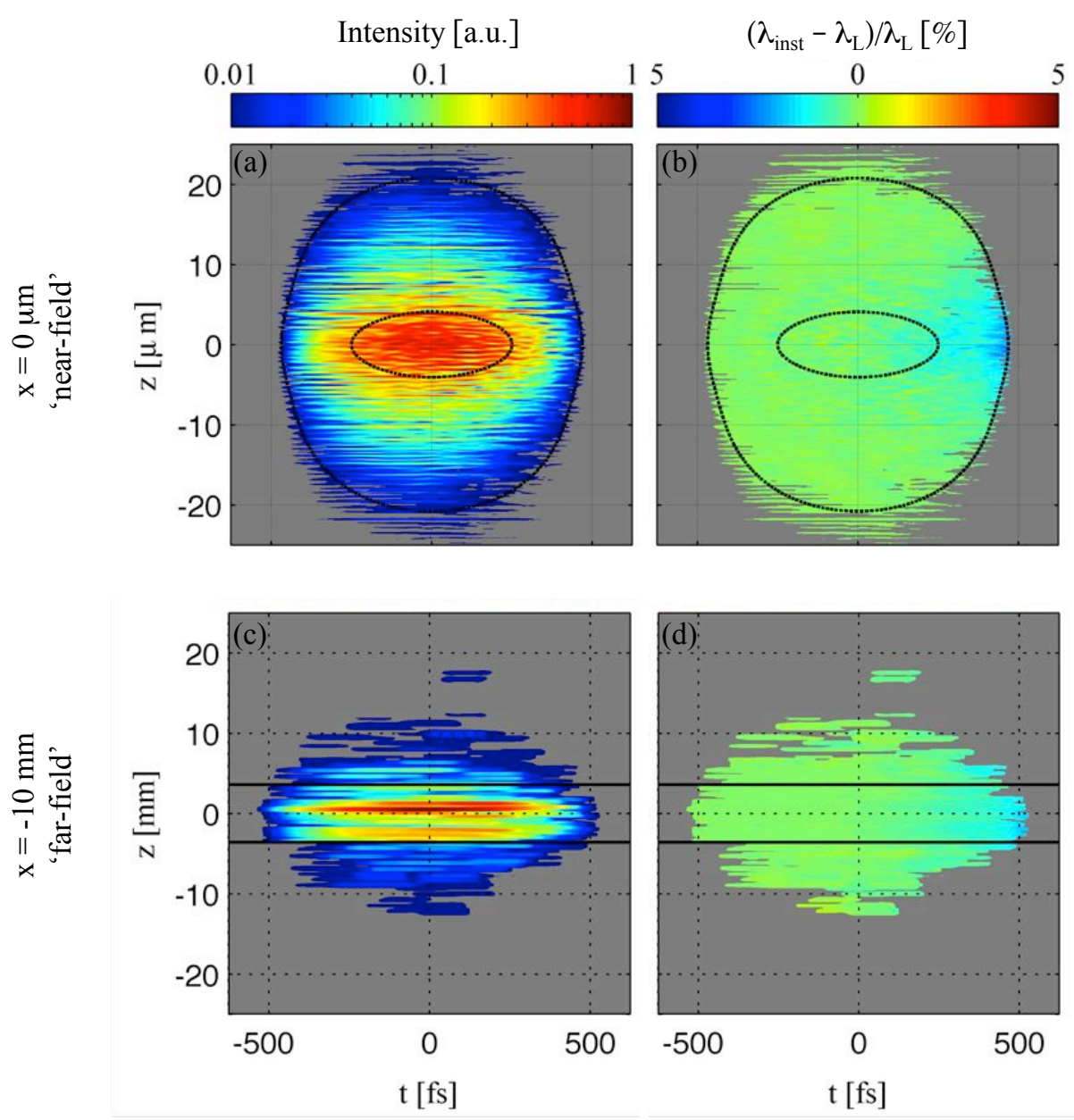

Figure 6.5: (Color) Spatially and temporally resolved intensity (first column) and instantaneous wavelength shifts (second column) in the near-field (top row) and far-field (bottom row). The dashed black contours in the near-field indicate the 1 and $50 \%$ peak intensity contours of the incident laser pulse and the solid black lines in the far-field indicate the collection window of the Spectralon ${ }^{\circledR}$ used in the experiment.

or with a finite sampling area (like those in the experiment discussed in Chapter 2) as these measurements will be indicative of the high-intensity, near-field shifting. Just like for the low-contrast interaction of Chapter 4 , we observe very little modifications to the temporal pulse duration as compared to the input. 
(a)

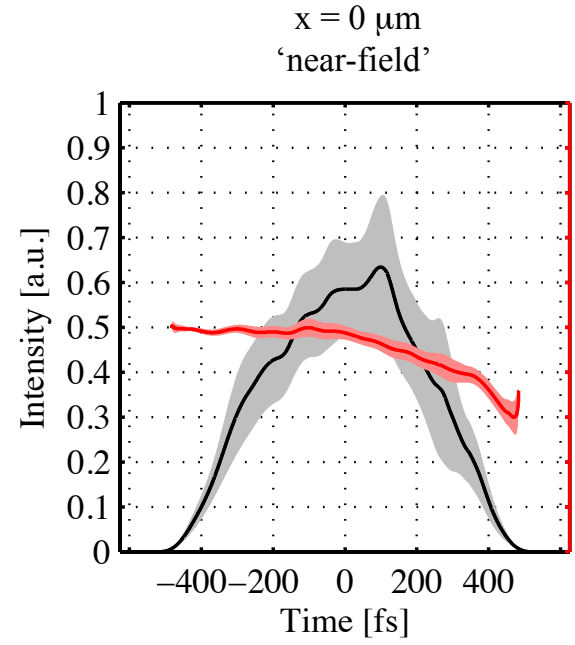

(b)

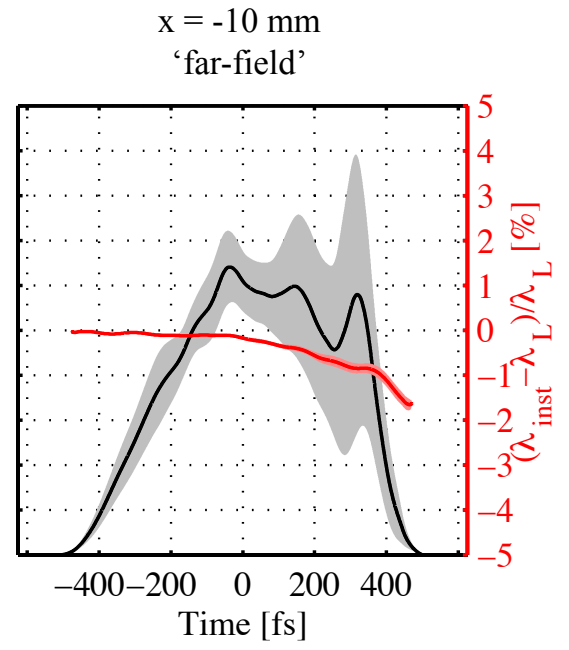

Figure 6.6: (Color) Spatially integrated, intensity weighted instantaneous intensity and wavelength shifts in the (a) near and (b) far-field. Solid black/red curves indicate the average values and shaded gray/pink areas indicate a $\pm \sigma_{S T D}$ about the average.

\section{Short pulse validity}

Unlike the previous low-contrast simulations, there is significantly less under-dense plasma present near the interaction region throughout these high-contrast simulations but we note that the relativistic critical surface has been altered by the falling half-peak intensity of the pulse. Using short $100 \mathrm{fs}$ pulses, we can study how the spatial scales of these different surface features affect specularly reflectivity with minimal distortion of the interaction surface or introduction of laser altering under-dense plasma phenomena. Additionally, shorter pulse simulations are computationally less taxing and would allow for a larger phase-space of surface perturbations to be tested. In this section, we address the validity of using these shorter test pulses.

A comparison of the short $(100 f s)$ and long $(500 f s)$ pulse spatially integrated spectra and instantaneous wavelength shifts of the specularly reflected pulses are shown in Figure 6.7 for the same surface above. The first row shows the spatially and temporally integrated spectra of the incident (black) and specular (red) pulses for the short and long pulses (left 
and right columns respectively). Unsurprisingly, the shorter pulse has a larger bandwidth than the longer pulse. For both cases, we see slight spectral broadening $(\approx 9 \%$ for the short pulse, $\approx 30 \%$ for the long pulse) and an overall blueshift ( $0.2 \%$ for the short pulse, $0.3 \%$ for the long pulse). Shown in the second row is the intensity weighted, spatially integrated instantaneous wavelength (red) and intensity (black) as a function of time. For the long pulse, the trailing edge has a stronger blueshift between 1 and $2 \%$ likely due to the enhanced heating of the interaction region with the longer pulse.
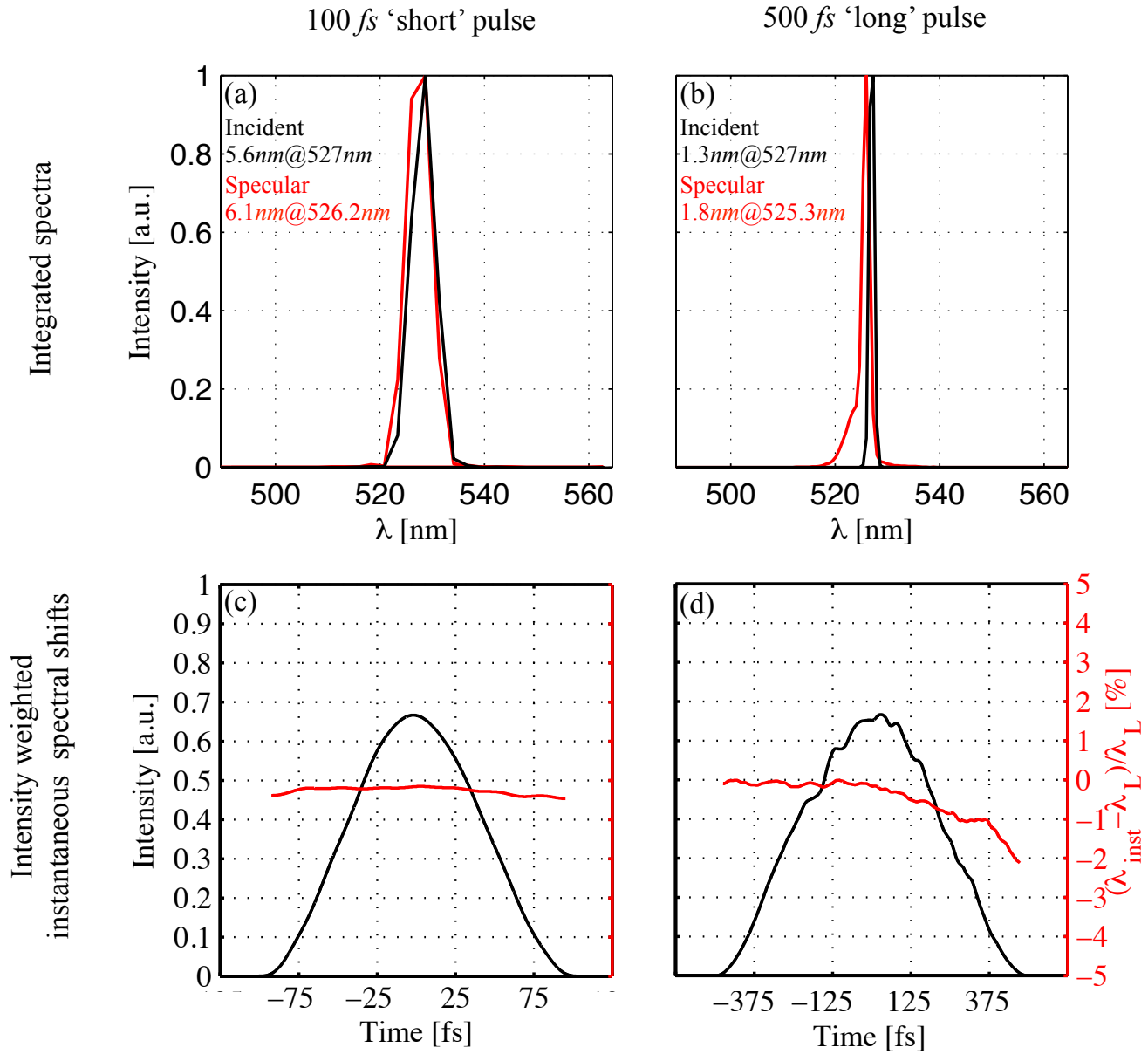

Figure 6.7: (Color) Spatially integrated spectra of the incident and reflected light for the (a) short and (b) long pulses. Spatially integrated, intensity weighted instantaneous wavelength shifts for the (c) short and (d) long pulses. 
Shown in Figure 6.8 are the near and far-field intensity distributions of the specularly reflected pulse (red) and the incident laser pulse (black). In the left column is the shorter test pulse with a $100 \mathrm{fs}$ pulse FWHM and on the right, the $500 \mathrm{fs}$ pulse characteristic of the experiment. The intensity distributions of the near-field plots (top row) have been normalized to the incident laser intensity to show spatial variations in absorption. ${ }^{41}$ Not only is the reflectivity lower for the longer pulse, the spatial intensity distribution is smoother. This is likely due to the smoothing of the critical surface from the under-dense plasma expansion. Interestingly, the ratio of the red to black curves show lower reflectivity from the higher intensity portions of the pulse, consistent with previous studies [90]. The far-field intensity distributions, propagated $10 \mathrm{~mm}$ away from the original laser focal plane, are shown in plots (c) and (d) in the bottom row (normalized by the peak). The solid angle of the detector in the experiment is also shown, indicated by the dahsed-black vertical lines. In each case, spatial diffraction and spectral broadening cause the experimental window $(\sim f / 2.5 \times 527 \pm 25 \mathrm{~nm})$ to only capture $75-85 \%$ of the unabsorbed light.

Because of the significant differences in spectra and coupling, shorter pulses are insufficient to study the spectral shifting observed in the experiment. We do find that these shorter pulses, however, are useful for providing insight into which scale of surface perturbations is driving different reflectivity phenomena. Using simulations, we can break down the realistic surface roughness into large and small scale features correspondingly see how each component is affecting the experimental observables (depicted in the first row of Figure 6.9). One of the realistic surface roughness with consistent spectral intensity distribution and same overall RMS as the target measurements was chosen in particular (200 $\mathrm{nmRMS}$, distribution 2, red surface). The (ai) realistic target surface was broken down into (bi) large and (ci) small scale features $\left(2 \lambda_{L}\right.$ edge filter).

Shown in the second row of Figure 6.9 are the normalized time integrated intensity distributions $10 \mathrm{~mm}$ away from the focal plane at a distance where the diffraction pattern has converged for each of these surfaces. The black curves in these three figures are the far-

\footnotetext{
${ }^{41}$ Yet again, note how well the non-paraxial propagation technique back to the original laser focal plane consistently fits inside the incident pulse envelope. The regions that exist outside this envelope are likely from focusing in concave regions of the surface features.
} 

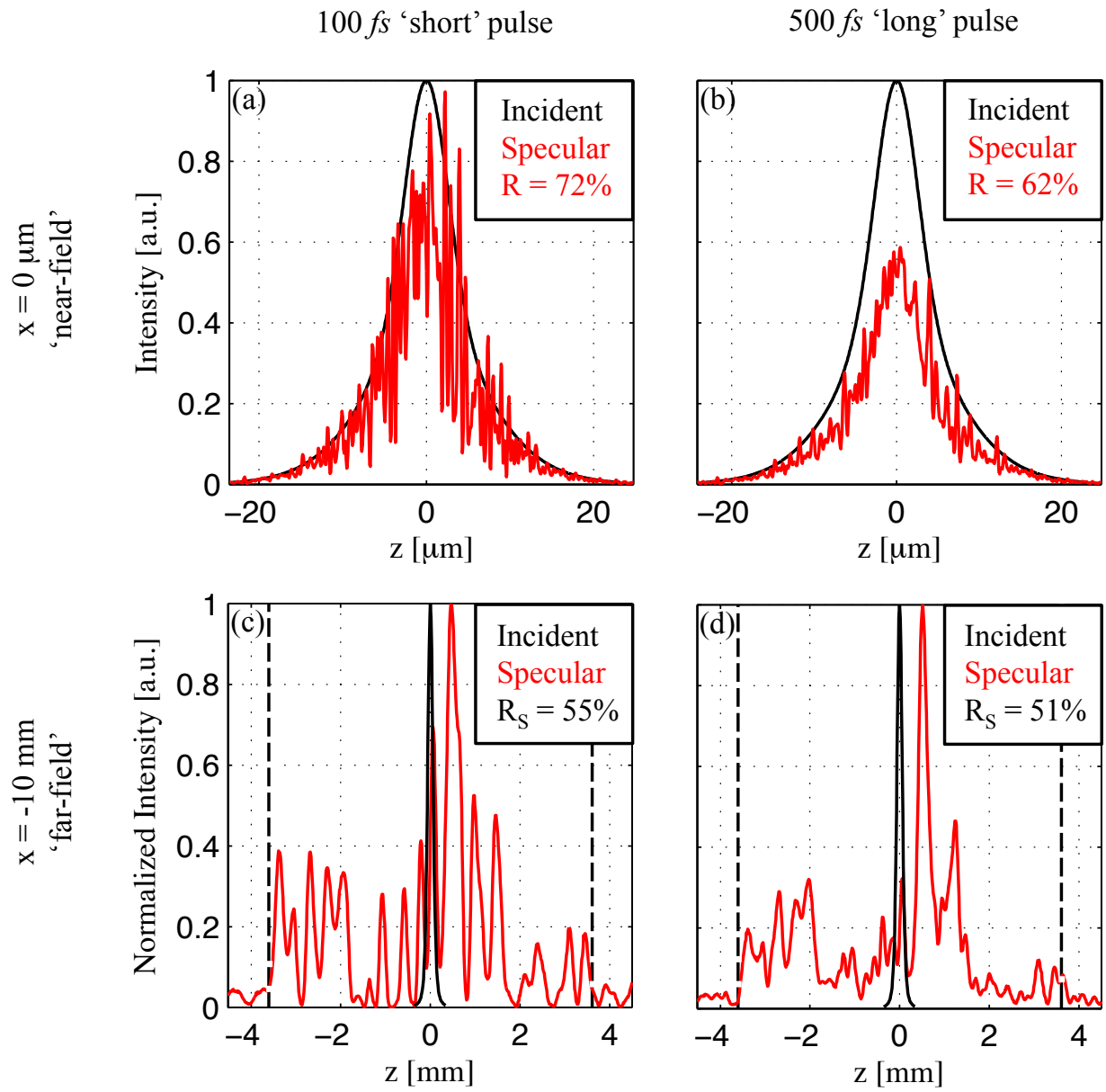

Figure 6.8: (Color) Time-integrated spatial intensity distributions of the specularly reflected pulse for both long and short pulses. Subplots (a) and (b) are the near-field (at best laser focus $x=0 \mu \mathrm{m})$ and (c) and (d) are the far-field $(x=-10 \mathrm{~mm})$ intensity distributions. Profiles for both the incident and specular pulses are indicated by the black and red curves respectively. The vertical black lines in the far-field plots indicate the solid angle of the experimental reflectivity measurement.

field intensity distributions for the perfectly flat interface, showing a mirror-like reflection with about $85 \%$ reflectivity. In (bii) we have only large scale features (red) which has very strong diffraction and spatial broadening due to the surface roughness with a slightly lower reflectivity of $78 \%$. In contrast, (cii) is from the simulation that only used the small scale features. As expected, the small scale, more-or-less optically-flat, target shows a mirror-like reflection but with a lower reflectivity of $70 \%$. The realistic target with a combination of 

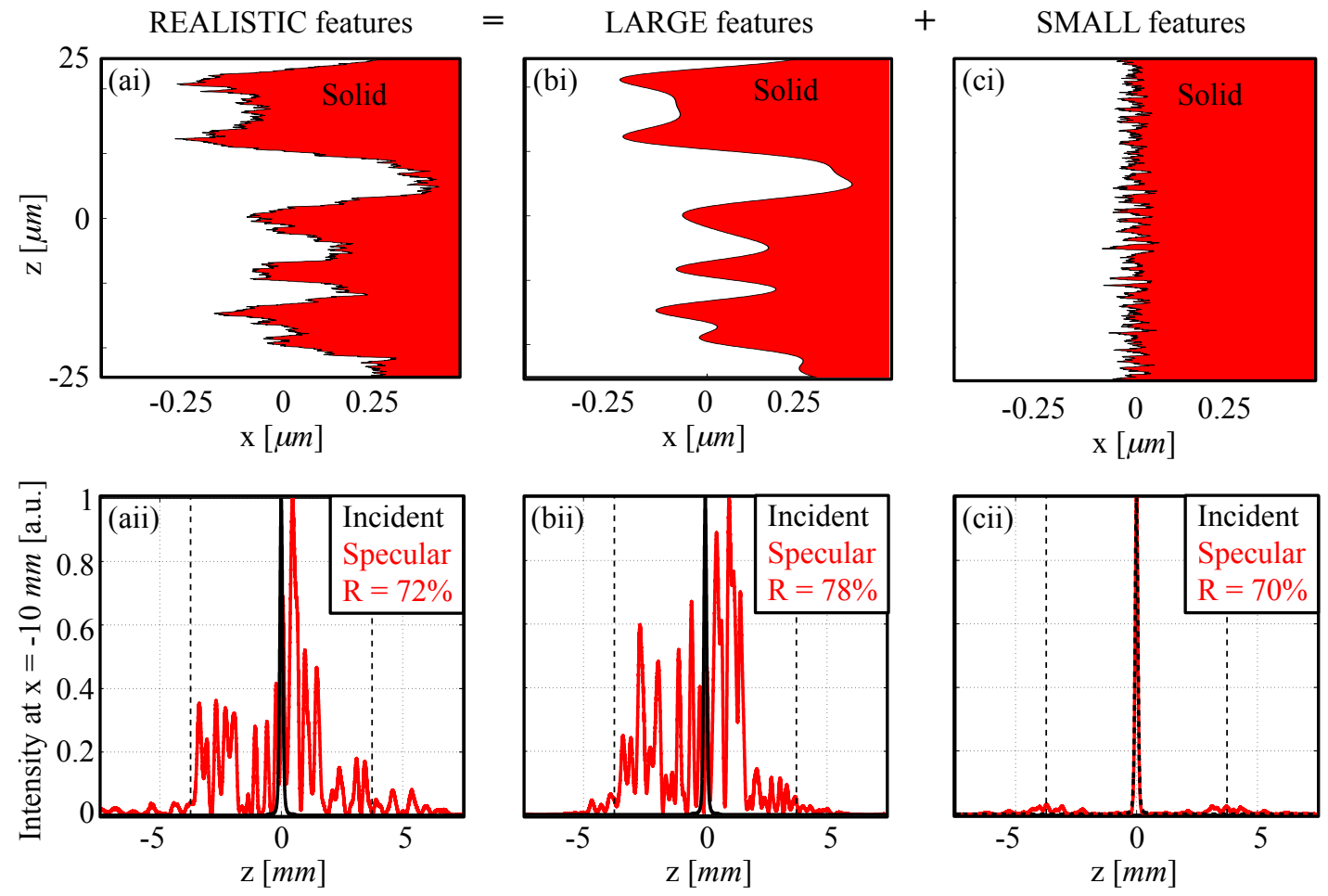

Figure 6.9: (Color) The 'realistic' surface roughness and corresponding far-field intensity distributions of the specularly reflected pulse from the simulation are shown in (ai) and (aii) respectively. Also shown in black are the far-field spatial intensity profiles of the incident laser pulse. Similar for just the 'large' scale features $\left(\lambda \geq 2 \lambda_{L}\right)$ and just the 'small' scale features $\left(\lambda<2 \lambda_{L}\right)$ in subplots (b) and (c) respectively.

both large and small features has a intensity distribution similar to the large scale, but a reflectivity closer to the small scale at $72 \%$. Consistent with the reflectivity data shown in Figure 2.16, the large scale features are expected to predominantly affect the far-field diffraction pattern of the specular pulse while the small i.e. optically-flat roughness has a minimal influence. Consistent with the simple sinusoidal surface, the coupling is highest for the only small features simulation. 


\subsection{Hot-electron sensitivity to surface features}

\subsubsection{Short pulse parameter scan}

Since a variety of surface distributions were observed in experiment, a parameter study of these various surfaces was performed using the recreated surfaces in Figure 6.2. This was done using the shorter (100 fs) pulse since for their computational convenience and simplified interaction since coupling into hot-electrons is dominated by standing-wave accelerations and not under-dense traveling-wave accelerations (allowing us to more easily focus on the electrons of interest). From the preliminary reflectivity simulations, we have reason to believe that the absorption is being strongly influenced by the sub-wavelength scale surface perturbations more than the spot-sized large features. To see the effect on the reflectivity and electrons, each surface distributions was run twice: (1) using 'Large Scale' features from the low resolutions AFM scans shown in Figure 6.2(a) and (2) 'Large+Small Scale' features which are a superposition of the high and low resolution AFM scans. The results of these shorter pulse simulations are summarized in Table 6.1. The surfaces have been identified by the colors defined by Figure 6.2. Although not characteristic of the experiment, the perfectly flat surface (black) and one with just small features (magenta) are also shown for reference. Both conversion efficiencies, defined to be the fraction of laser energy converted into hot-electrons with energy above $50 \mathrm{keV}$ that made to the diagnostic plane $x=5 \mu m$ deep inside solid density, are shown.

Table 6.1: Conversion efficiency of the various experimentally realistic surface features addressed in this study for $100 \mathrm{fs}$ FWHM pulses.

\begin{tabular}{ccccc}
\hline \hline Surface & RMS & Large scale distribution & \multicolumn{2}{c}{ Conversion efficiency, $\eta_{L \rightarrow e^{-}}[\%]$} \\
\cline { 4 - 5 }$($ Figure 6.2) & {$[\mathrm{nm}]$} & (Figure 6.2(a)) & Large scale & Large+small scale \\
\hline \hline blue & 400 & 1 & 2.4 & 3.8 \\
red & 200 & 2 & 8.7 & 11.3 \\
green & 100 & 2 & 6.7 & 10.9 \\
cyan & 100 & 3 & 5.9 & 8.9 \\
\hline flat & 0 & - & $4.2($ black $)$ & 18.2 (magenta) \\
\hline \hline
\end{tabular}


The corresponding hot-electron energy spectra are shown in Figure 6.10 for (a) only the large scale features and (b) the superposition of large and small scale features. Once again, they have been color coded to the surfaces according to Figure 6.2. Of the large scale features, we find that the blue surface (i.e. the isotropic distribution characteristic of the Goodfellow foils like in Figure 2.10(a)) has significantly lower coupling than even the perfectly flat surface. The other surfaces observed (blue, red, green and cyan) were characteristic of the electroplated targets and they all had similar conversion efficiencies and electron spectra. With the addition of the small scale features, the optically flat surface saw more than a $4 \times$ increase in conversion efficiency over the perfectly flat surface. The addition of the sub-wavelength features increased the coupling into hot electrons independent of the large scale features, however, the surfaces that were characteristic of the experiment didn't even double the conversion efficiency over the only large feature surfaces. In fact, the optically flat target had the largest conversion efficiency of all the surfaces considered. For all of these surfaces, the majority of total conversion efficiency (>95\%) was into standingwave electrons, i.e. electrons with energy below the standing wave quiver energy $U_{p}^{S W}=$ $\left(\gamma_{o}^{S W}-1\right) m_{e} c^{2}=2.8 \mathrm{MeV}$ where the peak relativistic Lorentz factor $\gamma_{o}^{S W}=\sqrt{1+\left(2 a_{o}\right)^{2}}=$ 6.5 .

A few representative angular distributions, resolved by energy, each energy bin individually normalized to the peak, are shown in Figure 6.11. All the angular distributions looked similar to the optically-flat sinusoidal surface perturbations in Figure 5.4 but with less than half the conversion efficiency of the simple sinusoidal surface. From the work in the previous chapter, all these factors would suggest that the severity of the perturbations in the standing-wave wavefronts from the large scale features is driving deviations from the standing-wave acceleration mechanism resulting in more stochastic, less efficient, electron accelerations.

\subsubsection{Long pulse differences}

From the experimental reflectivity measurements (Chapter 2.5) and simulations with simplified surface perturbations (Chapter 5), the case that surface perturbations can play a 
(a) Only large features

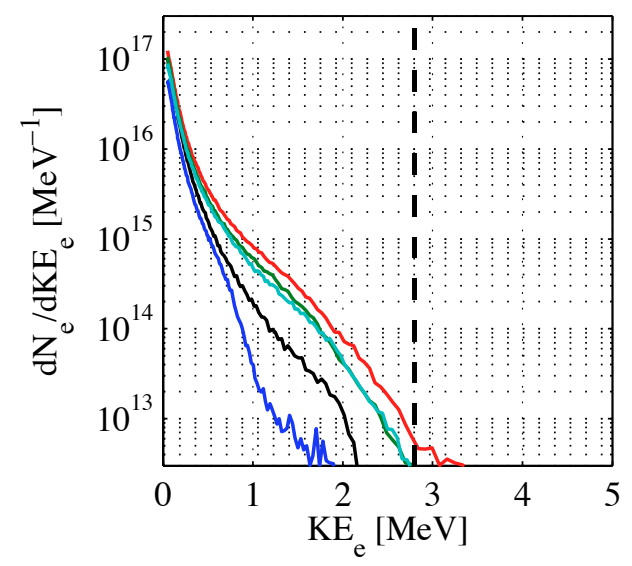

(b) Large \& small features

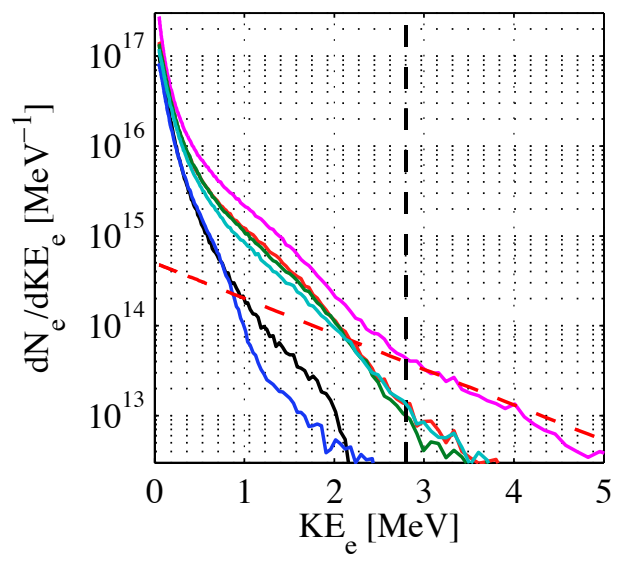

Figure 6.10: (Color) Variation in hot-electron energy spectra, $5 \mu \mathrm{m}$ inside solid density, generated from the $100 \mathrm{fs}$ pulse for the various color coded surface features in Figure 6.2. (a) Spectra from surfaces that only had large features and (b) superposition of large and small features. Also shown in both plots are the spectra generated at a perfectly flat interface (black), from the small scale features only (magenta), the standing-wave quiver energy $U_{p}^{S W}=2.8 \mathrm{MeV}$ (dashed - black) and a Boltzmann distribution with temperature equal to the traveling-wave quiver energy, $T_{e}=U_{p}^{T W}=1.1 \mathrm{MeV}($ dashed - red $)$.

significant role in the high-contrast interactions is quite strong. With a longer pulse, however, more laser energy couples into the target, further heating the target to the point where a significant under-dense plasma can form on the front of the target. At this point, coupling very quickly becomes dominated by the traveling-wave accelerated electrons originating from under-dense regions as these electrons, although fewer in number, can extract a significant fraction of energy from the laser. Shown in Figure 6.12(a) is the energy spectrum on a $\log _{10}-\log _{10}$ scale of the hot-electrons generated in the long pulse interaction for the red surface encompassing both large and small scale features. The short pulse spectrum is also shown, for reference, in black and the peak standing-wave quiver energy is indicated by the dashed-black vertical line. For the longer $500 \mathrm{fs}$ pulses characteristic of the experiment, coupling into hot-electrons jumped to $25.5 \%$ (over the $\lesssim 12 \%$ ) where $\sim 6 \%$ of the laser energy was converted into a Wilks-like hot-tail (dashed-red curve). The corresponding 
(a) Flat interface (black)

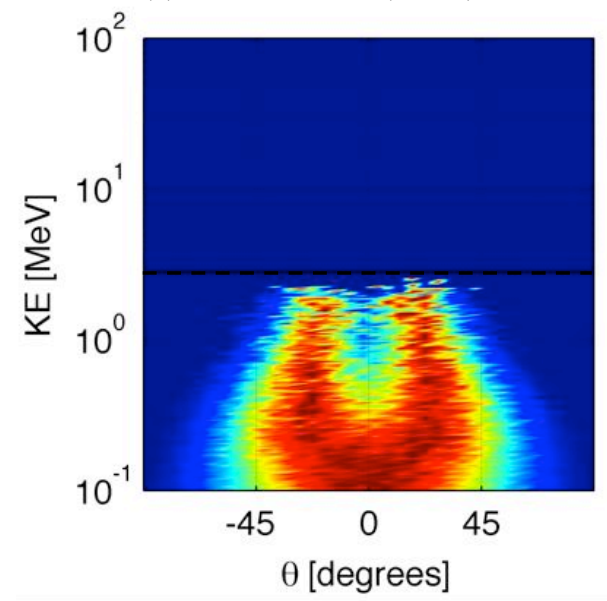

(c) Only large features (red)

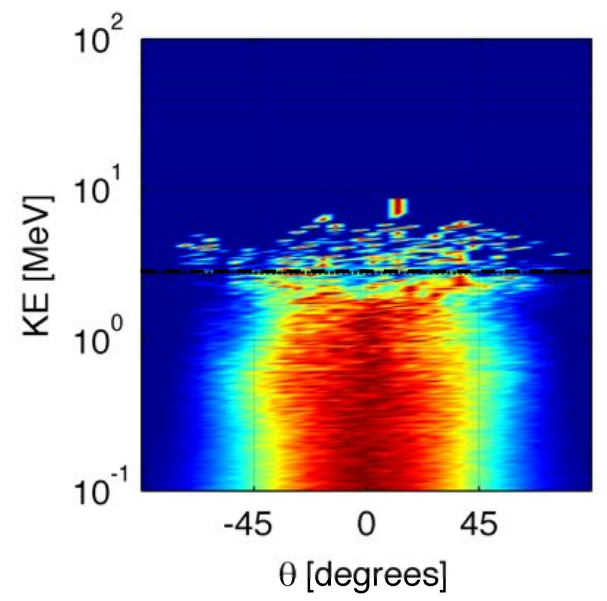

(b) Only small features (magenta)

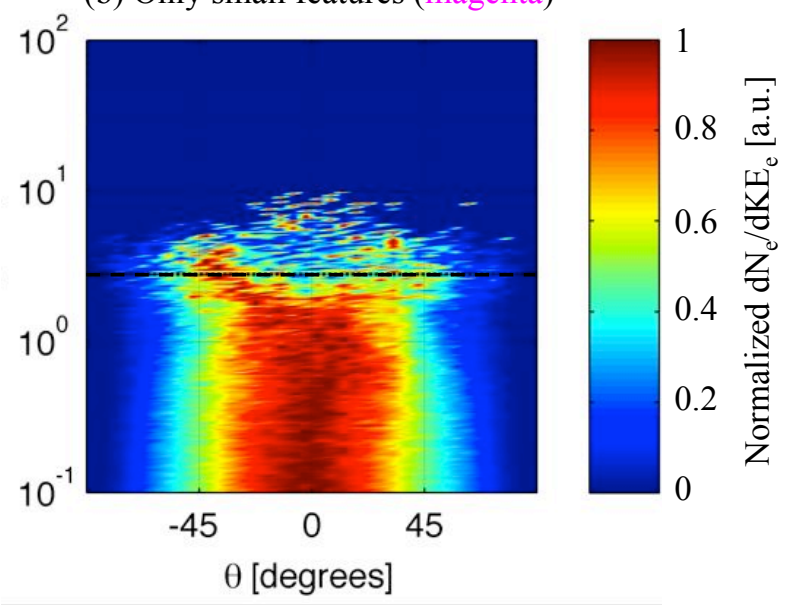

(d) Large \& small features (red)

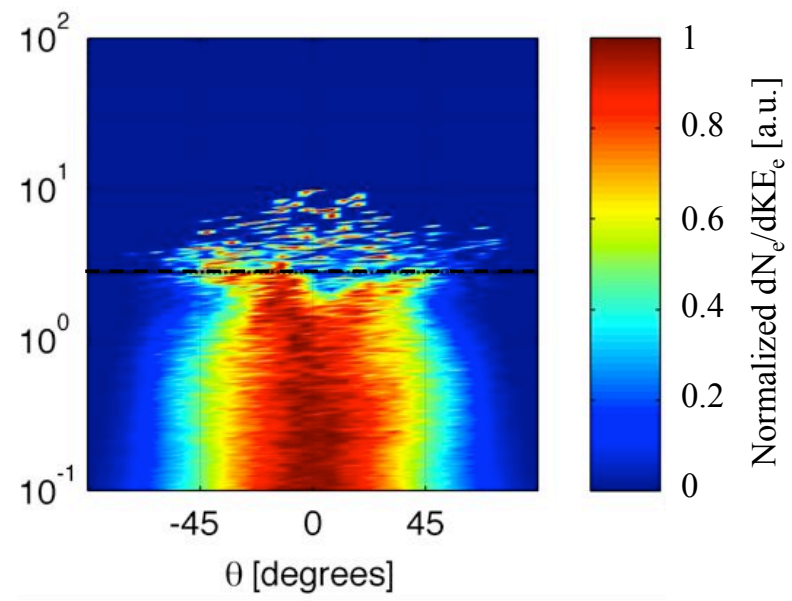

Figure 6.11: (Color) Angular distribution of hot-electrons that cross the diagnostic plane at $x=5 \mu \mathrm{m}$ inside solid density for various surfaces, resolved by energy with each energy bin individually normalized. (a) The perfectly flat interface (black), (b) only small scale features (magenta), (c) only large scale features of red surface and (d) both large and small scale features of red. The standing-wave quiver energy $U_{p}^{S W}=2.8 \mathrm{MeV}$ is also indicated by the horizontal dashed - black lines.

normalized angular distributions are shown in (b), where these supra- $U_{p}^{S W}$ electrons are reasonably characterized by the classical ejection angle (dashed-white curve), indicative of the plane-wave acceleration. 

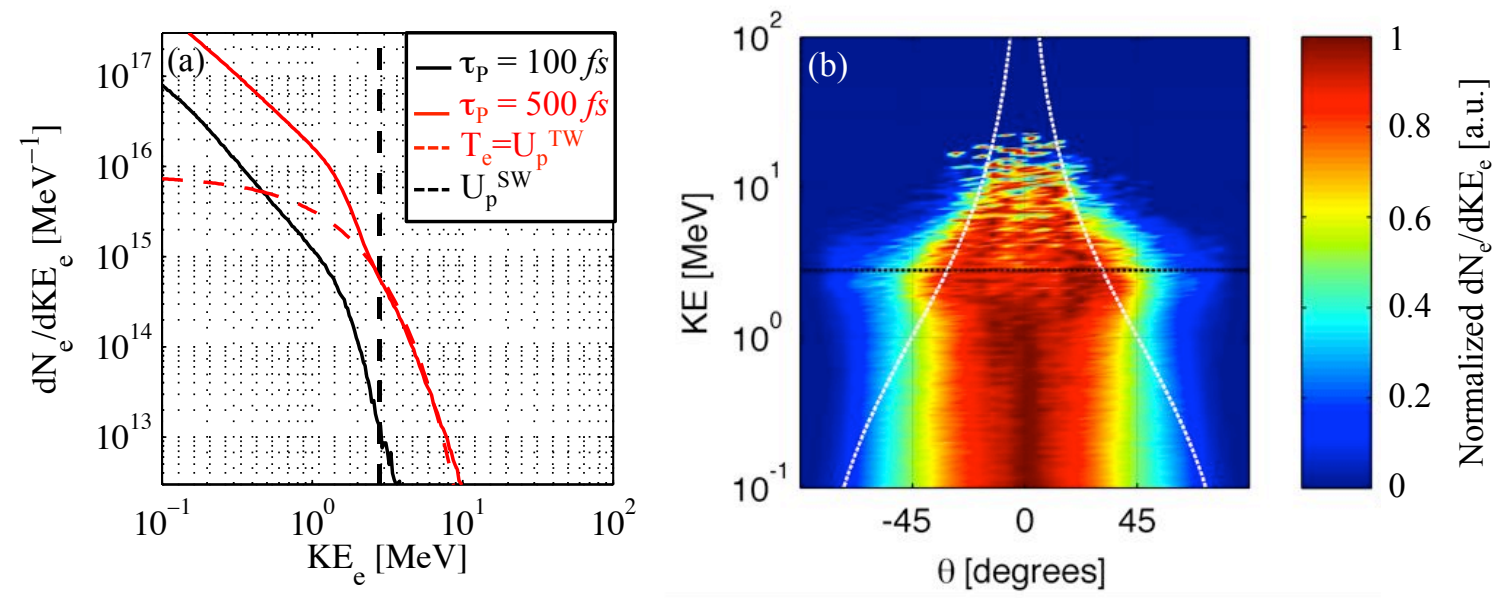

Figure 6.12: (Color) (a) Integrated energy and (b) angular distribution of hot-electrons that cross the diagnostic plane at $x=5 \mu \mathrm{m}$ inside solid density for the red surface. In (a), the short pulse distribution is given by the solid black curve, the solid red curve is the long pulse result and the dashed red curve indicate a Boltzmann distribution with temperature equal to the traveling-wave quiver energy $U_{p}^{T W}=1.1 \mathrm{MeV}$. The dashed-black lines in both figures indicate the standing-wave quiver energy $U_{p}^{S W}=2.8 \mathrm{MeV}$. In (b), the dashedwhite curves indicate the classical ejection angle for an electron accelerated by a plane wave (starting from rest).

\subsection{Summary}

High-contrast relativistic laser-plasma interactions, both in terms of hot-electron generation and specular reflectivity measurements, are strongly influenced by surface perturbations. When comparing to experimental data in Chapter 2, we find that the experimentally realistic recreated surface roughnesses produce very little spectral shifting due to the absence of significant pre-plasma. While not as physically interesting as the elaborate shifting observed in the low-contrast interactions, these measurements provide a good indicator of the extent to which the interaction remains high-contrast. Spatial uniformity in the far-field propagation indicate that even spatially sub-sampled measurements would be indicative of the near-field physics. Similar far-field diffraction patterns to the $f / 20 \mathrm{CW}$ from experiment (Figure 2.16) were observed instead of what was characteristic of the $f / 2.5$ short pulse. 
This is likely because the simulation used a $\sim f / 40$ Gaussian beam, but indicates that the surface roughnesses simulated were a reasonable approximation of what was observed in experiment.

For the short pulse, coupling of laser energy into hot-electrons is predominantly into standing-wave accelerated electrons (with energy $\lesssim U_{p}^{S W}=2.8 \mathrm{MeV}$ ) since the interface remains sharp throughout. Because of bandwidth and coupling differences, shorter pulses are insufficient to study the long pulse shifting phenomenon but they did provide a less expensive means of studying the phase space of surface perturbations shot during the experiment. The small scale features, despite only having a $25 \mathrm{~nm}$ RMS, appeared to dominate the absorption physics while the large scale predominantly created more stochastic fields and drove far-field diffraction. Most of the targets produced produced very similar hot-electron characteristics, predominantly driven by standing-wave accelerations, despite the variability in surface perturbations. For the longer pulse, like those used in the experiment, eventual under-dense plasma expansion resulted in longer acceleration distances and hot-electrons characteristic of traveling-wave accelerations, dominating the spectrum above $U_{p}^{S W}$ 


\section{Chapter 7 Conclusions}

In conclusion, I have studied low and high-contrast relativistic laser-plasma interactions using analytic descriptions, experimental observations and particle-in-cell modeling in the context of hot-electron generation. Since these hot-electrons cannot be directly observed in experiment, I utilized the often neglected specularly reflected diagnostics to constrain simulations used to infer hot-electron properties. ${ }^{42}$ In particular, all the specular reflectivity diagnostics suggest that the high-contrast interactions were particularly sensitive to initial target surface perturbations which, to some extent, survived throughout the interaction. In either case, I find these interactions to be highly sensitive to initial target conditions and spatial/temporal pulse properties. For a quantitative comparison to experiment, both the conditions must be simultaneously met in simulations (something not yet accomplished in this work). While approximations of the incident laser pulse made quantitative comparison difficult for this work, all of the experimental trends were qualitatively reproduced.

For low-contrast interactions, non-linear interactions between the laser and under-dense plasma drive pulse altering phenomena and lead to a dynamically evolving interaction region. While many of these effects can be inferred from specular measurements, diffraction of the pulse in the far-field can lead to spatially varying observations that are not always indicative of the near-field physics. The far-field analysis was performed using a novel non-paraxial field propagation technique which was found to reliably propagate spectral modes up to the laser wavelength. For these aluminum targets, dynamic ionization in the

\footnotetext{
${ }^{42}$ In ideal world, all transport and specular diagnostics would simultaneously be used to constrain simulations. This is a work in progress for these simulations.
} 
simulated pre-plasma environment was found to significantly influence the instantaneous wavelength shifts of the specularly reflected pulse, resulting in simulated shifts similar to the experimental data but with only subtle differences in the hot-electron spectra and angular distributions with the fixed ionization simulation. Similar to previous works, I find that the instantaneous wavelength shifting of the specularly reflected pulse is dominated by the Doppler shift associated with the motion of the relativistic critical surface. Coupling into hot-electrons is dominated by a super-hot component with energies well beyond the quiver energy that are characteristic of traveling-wave accelerations. However, non-linear laser-plasma instabilities and quasi-static magnetic field generation from the under-dense plasma can create less predictable interactions and significantly perturb even the relativistic electron trajectories (See Figure 7.1).

(a) High-contrast

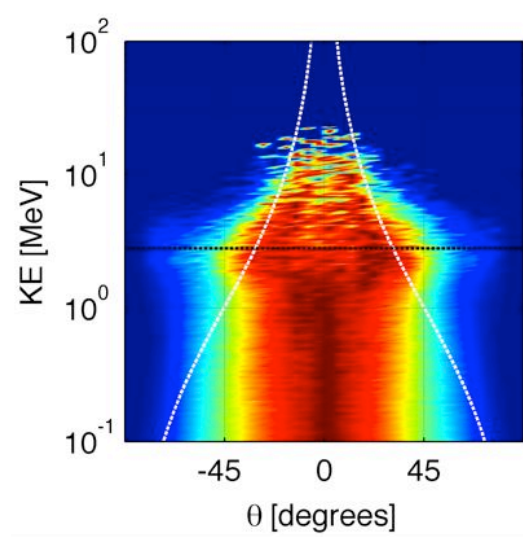

(b) Low-contrast fixed ionization

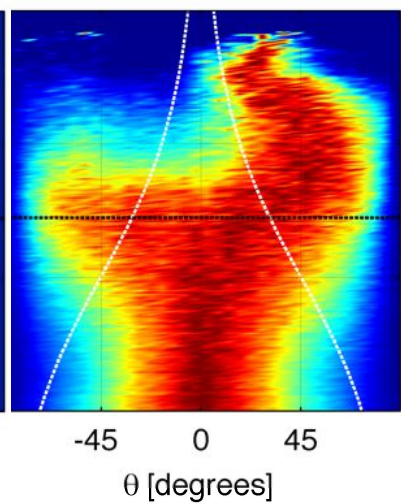

(c) Low-contrast field ionization

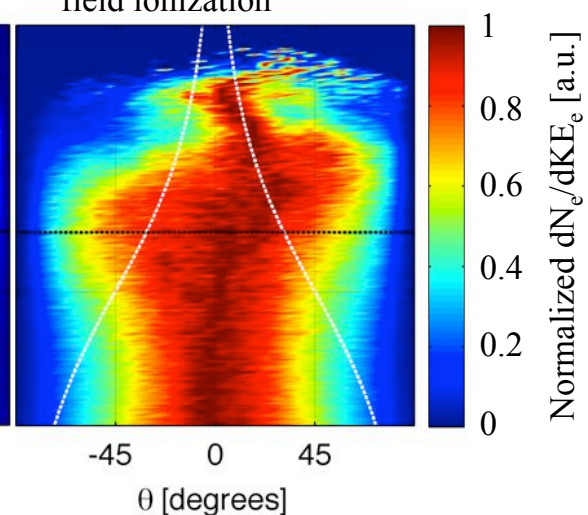

Figure 7.1: (Color) Comparison of spatially and temporally integrated hot-electron angular distributions, resolved by kinetic energy, for the (a) high-contrast simulation, (b) fixed ionization and (c) field ionization simulations.

High-contrast interactions are found to be highly sensitive to the scale of surface features in both experiment and simulation. For perfectly flat interfaces, I find that electrons generated throughout the interaction are characteristic of the standing-wave acceleration mechanism, but only when the plasma skin effect in included. This is a critical distinction 
from previous works in that it describes how ultra-intense lasers can accelerate low energy electrons to relativistic energies from initially cold targets with this mechanism. The unique bifurcation signature 'washes out' at late times and is typically attributed to growing quasi-static fields inside solid density, but I find this phenomena to be more consistent with target heating. With even the addition of 'optically flat' surface perturbations, significant enhancements in coupling are observed and these quasi-static fields appear to be beneficial in that they can recirculate low energy electrons back into the vacuum laser fields. Unlike traditional coupling mechanisms, this 'surfing' of electrons around the interface by the competing effects of magnetic field recirculation and direct laser acceleration present the electron multiple opportunities to gain a relativistic kick from the laser, at which point it escapes the trapping and propagates deep into the solid density target. For rougher targets, increased coupling results in extensive under-dense plasma expansion and electrons no longer characteristic of the standing-wave mechanism. Depending on the under-dense plasma expansion, the absorption enhancing features can be smoothed over by the motion of the relativistic critical surface. Increasingly structured interfaces can drive chaotic field patterns near the interface, resulting in more stochastic electron accelerations and broader angular distributions.

Despite having overall lower coupling compared to the low-contrast interaction, highcontrast interactions predominantly couple laser energy into electrons that are characteristic of the standing-wave acceleration mechanism. In fact, under these conditions, the high-contrast interaction showed increased coupling into sub-quiver energy electrons over the low-contrast simulations (nearly $3 \times$ ). Additionally, because of the limited under-dense plasma, quasi-static fields generated near the interaction interface and non-linear laserplasma instabilities are significantly less pronounced, resulting in more consistent and predicable hot-electron characteristics. Depending on the application, consistency and directionality may be more important than overall laser coupling which is typically dominated by higher energy, traveling-wave accelerated electrons (see Figure 7.2). 


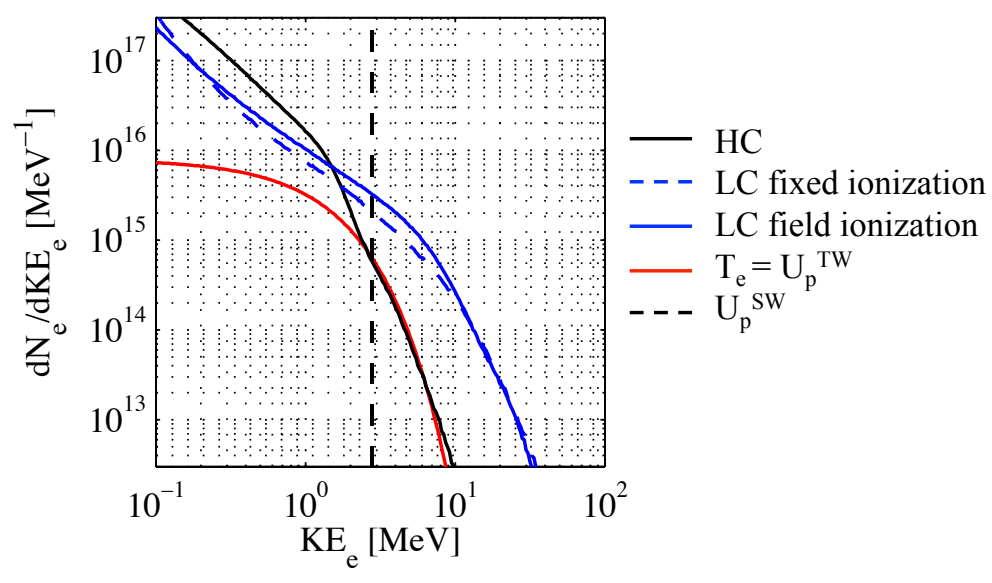

Figure 7.2: (Color) Spatially and temporally integrated hot-electron energy spectra for the fixed ionization (red) and field ionization (black) simulations. Also shown is the spectrum from the same high-contrast laser pulse interaction with the realistic surface roughness discussed in Chapter 6. 


\section{BIBLIOGRAPHY}

[1] Max Tabak, James Hammer, Michael E. Glinsky, William L. Kruer, Scott C. Wilks, John Woodworth, E. Michael Campbell, Michael D. Perry, and Rodney J. Mason. Ignition and high gain with ultrapowerful lasers. Physics of Plasmas, 1(5):1626-1634, 1994.

[2] P. K. Patel, A. J. Mackinnon, M. H. Key, T. E. Cowan, M. E. Foord, M. Allen, D. F. Price, H. Ruhl, P. T. Springer, and R. Stephens. Isochoric heating of solid-density matter with an ultrafast proton beam. Phys. Rev. Lett., 91:125004, Sep 2003.

[3] E. Brambrink, H. G. Wei, B. Barbrel, P. Audebert, A. Benuzzi-Mounaix, T. Boehly, T. Endo, C. D. Gregory, T. Kimura, R. Kodama, N. Ozaki, H.-S. Park, and M. Koenig. Direct density measurement of shock-compressed iron using hard x rays generated by a short laser pulse. Phys. Rev. E, 80:056407, Nov 2009.

[4] Donald Umstadter. Relativistic laser-plasma interactions. Journal of Physics D: Applied Physics, 36(8):R151, 2003.

[5] Madelon Pijls-Johannesma, Janneke P.C. Grutters, Frank Verhaegen, Philippe Lambin, and Dirk De Ruysscher. Do we have enough evidence to implement particle therapy as standard treatment in lung cancer? a systematic literature review. The Oncologist, 15(1):93-103, 2010.

[6] Bruce A. Remington, R. Paul Drake, and Dmitri D. Ryutov. Experimental astrophysics with high power lasers and $z$ pinches. Rev. Mod. Phys., 78:755-807, Aug 2006. 
[7] R.P. Drake, L. Davison, and Y. Horie. High-Energy-Density Physics: Fundamentals, Inertial Fusion, and Experimental Astrophysics. Shock wave and high pressure phenomena. Springer London, Limited, 2006.

[8] John Lindl. Development of the indirect-drive approach to inertial confinement fusion and the target physics basis for ignition and gain. Physics of Plasmas, 2(11):39334024, 1995.

[9] Steven W. Haan, Stephen M. Pollaine, John D. Lindl, Laurance J. Suter, Richard L. Berger, Linda V. Powers, W. Edward Alley, Peter A. Amendt, John A. Futterman, W. Kirk Levedahl, Mordecai D. Rosen, Dana P. Rowley, Richard A. Sacks, Aleksei I. Shestakov, George L. Strobel, Max Tabak, Stephen V. Weber, George B. Zimmerman, William J. Krauser, Douglas C. Wilson, Stephen V. Coggeshall, David B. Harris, Nelson M. Hoffman, and Bernhard H. Wilde. Design and modeling of ignition targets for the national ignition facility. Physics of Plasmas, 2(6):2480-2487, 1995.

[10] D. B. Sinars, M. E. Cuneo, G. R. Bennett, D. F. Wenger, L. E. Ruggles, M. F. Vargas, J. L. Porter, R. G. Adams, D. W. Johnson, K. L. Keller, P. K. Rambo, D. C. Rovang, H. Seamen, W. W. Simpson, I. C. Smith, and S. C. Speas. Monochromatic Xray backlighting of wire-array z-pinch plasmas using spherically bent quartz crystals. Review of Scientific Instruments, 74(3):2202-2205, 2003.

[11] O. Ciricosta, S. M. Vinko, H.-K. Chung, B.-I. Cho, C. R. D. Brown, T. Burian, J. Chalupský, K. Engelhorn, R. W. Falcone, C. Graves, V. Hájková, A. Higginbotham, L. Juha, J. Krzywinski, H. J. Lee, M. Messerschmidt, C. D. Murphy, Y. Ping, D. S. Rackstraw, A. Scherz, W. Schlotter, S. Toleikis, J. J. Turner, L. Vysin, T. Wang, B. Wu, U. Zastrau, D. Zhu, R. W. Lee, P. Heimann, B. Nagler, and J. S. Wark. Direct measurements of the ionization potential depression in a dense plasma. Phys. Rev. Lett., 109:065002, Aug 2012.

[12] S. N. Chen, G. Gregori, P. K. Patel, H.-K. Chung, R. G. Evans, R. R. Freeman, E. Garcia Saiz, S. H. Glenzer, S. B. Hansen, F. Y. Khattak, J. A. King, A. J. Mackin- 
non, M. M. Notley, J. R. Pasley, D. Riley, R. B. Stephens, R. L. Weber, S. C. Wilks, and F. N. Beg. Creation of hot dense matter in short-pulse laser-plasma interaction with tamped titanium foils. Physics of Plasmas, 14(10):102701, 2007.

[13] F. Perez, L. Gremillet, M. Koenig, S. D. Baton, P. Audebert, M. Chahid, C. Rousseaux, M. Drouin, E. Lefebvre, T. Vinci, J. Rassuchine, T. Cowan, S. A. Gaillard, K. A. Flippo, and R. Shepherd. Enhanced isochoric heating from fast electrons produced by high-contrast, relativistic-intensity laser pulses. Phys. Rev. Lett., 104:085001, Feb 2010.

[14] J. Nuckolls, L. Wood, A. Thiessen, and G. Zimmerman. Laser Compression of Matter to Super-High Densities: Thermonuclear (CTR) Applications. Nature, 239:139-142, sep 1972 .

[15] John Nuckolls, John Emmett, and Lowell Wood. Laser-induced thermonuclear fusion. Physics Today, 26(8):46-53, 1973.

[16] C.M. Braams and P.E. Stott. Nuclear Fusion: Half a Century of Magnetic Confinement Fusion Research. SERIES ON PLASMA PHYSICS. Taylor \& Francis, 2010.

[17] Stefano Atzeni and Jürgen Meyer-ter Vehn. The Physics of Inertial Fusion: BeamPlasma Interaction, Hydrodynamics, Hot Dense Matter: BeamPlasma Interaction, Hydrodynamics, Hot Dense Matter. Number 125. Clarendon Press, 2004.

[18] Mordecai D. Rosen. The physics issues that determine inertial confinement fusion target gain and driver requirements: A tutorial. Physics of Plasmas, 6(5):1690-1699, 1999.

[19] J. Meyer-Ter-Vehn. On energy gain of fusion targets: the model of kidder and bodner improved. Nuclear Fusion, 22(4):561, 1982.

[20] Stephen E. Bodner, Denis G. Colombant, John H. Gardner, Robert H. Lehmberg, Stephen P. Obenschain, Lee Phillips, Andrew J. Schmitt, John D. Sethian, Robert L. McCrory, Wolf Seka, Charles P. Verdon, James P. Knauer, Bedros B. Afeyan, and 
Howard T. Powell. Direct-drive laser fusion: Status and prospects. Physics of Plasmas, $5(5): 1901-1918,1998$.

[21] R.L. McCrory, R.E. Bahr, R. Betti, T.R. Boehly, T.J.B. Collins, R.S. Craxton, J.A. Delettrez, W.R. Donaldson, R. Epstein, J. Frenje, V.Yu. Glebov, V.N. Goncharov, O.V. Gotchev, R.Q. Gram, D.R. Harding, D.G. Hicks, P.A. Jaanimagi, R.L. Keck, J.H. Kelly, J.P. Knauer, C.K. Li, S.J. Loucks, L.D. Lund, F.J. Marshall, P.W. McKenty, D.D. Meyerhofer, S.F.B. Morse, R.D. Petrasso, P.B. Radha, S.P. Regan, S. Roberts, F. Sguin, W. Seka, S. Skupsky, V.A. Smalyuk, C. Sorce, J.M. Soures, C. Stoeckl, R.P.J. Town, M.D. Wittman, B. Yaakobi, and J.D. Zuegel. Omega icf experiments and preparation for direct drive ignition on nif. Nuclear Fusion, 41(10):1413, 2001.

[22] J. D. Kilkenny, S. G. Glendinning, S. W. Haan, B. A. Hammel, J. D. Lindl, D. Munro, B. A. Remington, S. V. Weber, J. P. Knauer, and C. P. Verdon. A review of the ablative stabilization of the rayleigh-taylor instability in regimes relevant to inertial confinement fusion. Physics of Plasmas, 1(5):1379-1389, 1994.

[23] V. A. Thomas and R. J. Kares. Drive asymmetry and the origin of turbulence in an icf implosion. Phys. Rev. Lett., 109:075004, Aug 2012.

[24] C. Bellei, P. A. Amendt, S. C. Wilks, M. G. Haines, D. T. Casey, C. K. Li, R. Petrasso, and D. R. Welch. Species separation in inertial confinement fusion fuels. Physics of Plasmas, 20(1):012701, 2013.

[25] R. B. Stephens, R. A. Snavely, Y. Aglitskiy, F. Amiranoff, C. Andersen, D. Batani, S. D. Baton, T. Cowan, R. R. Freeman, T. Hall, S. P. Hatchett, J. M. Hill, M. H. Key, J. A. King, J. A. Koch, M. Koenig, A. J. MacKinnon, K. L. Lancaster, E. Martinolli, P. Norreys, E. Perelli-Cippo, M. Rabec Le Gloahec, C. Rousseaux, J. J. Santos, and F. Scianitti. $k_{\alpha}$ fluorescence measurement of relativistic electron transport in the context of fast ignition. Phys. Rev. E, 69:066414, Jun 2004. 
[26] J. S. Green, V. M. Ovchinnikov, R. G. Evans, K. U. Akli, H. Azechi, F. N. Beg, C. Bellei, R. R. Freeman, H. Habara, R. Heathcote, M. H. Key, J. A. King, K. L. Lancaster, N. C. Lopes, T. Ma, A. J. MacKinnon, K. Markey, A. McPhee, Z. Najmudin, P. Nilson, R. Onofrei, R. Stephens, K. Takeda, K. A. Tanaka, W. Theobald, T. Tanimoto, J. Waugh, L. Van Woerkom, N. C. Woolsey, M. Zepf, J. R. Davies, and P. A. Norreys. Effect of laser intensity on fast-electron-beam divergence in soliddensity plasmas. Phys. Rev. Lett., 100:015003, Jan 2008.

[27] C. D. Chen, P. K. Patel, D. S. Hey, A. J. Mackinnon, M. H. Key, K. U. Akli, T. Bartal, F. N. Beg, S. Chawla, H. Chen, R. R. Freeman, D. P. Higginson, A. Link, T. Y. Ma, A. G. MacPhee, R. B. Stephens, L. D. Van Woerkom, B. Westover, and M. Porkolab. Bremsstrahlung and $\mathrm{k}$ alpha fluorescence measurements for inferring conversion efficiencies into fast ignition relevant hot electrons. Physics of Plasmas, 16(8):082705, 2009.

[28] B. Westover, C. D. Chen, P. K. Patel, M. H. Key, H. McLean, R. Stephens, and F. N. Beg. Fast electron temperature and conversion efficiency measurements in laserirradiated foil targets using a bremsstrahlung x-ray detector. Physics of Plasmas, 18(6):063101, 2011.

[29] C. Bellei, L. Divol, A. J. Kemp, M. H. Key, D. J. Larson, D. J. Strozzi, M. M. Marinak, M. Tabak, and P. K. Patel. Fast ignition: Dependence of the ignition energy on source and target parameters for particle-in-cell-modelled energy and angular distributions of the fast electrons. Physics of Plasmas, 20(5):052704, 2013.

[30] C. Ren, M. Tzoufras, F. S. Tsung, W. B. Mori, S. Amorini, R. A. Fonseca, L. O. Silva, J. C. Adam, and A. Heron. Global simulation for laser-driven mev electrons in fast ignition. Phys. Rev. Lett., 93:185004, Oct 2004.

[31] A Debayle, J J Honrubia, E d'Humires, and V T Tikhonchuk. Characterization of laser-produced fast electron sources for fast ignition. Plasma Physics and Controlled Fusion, 52(12):124024, 2010. 
[32] S Atzeni, A Schiavi, and J R Davies. Stopping and scattering of relativistic electron beams in dense plasmas and requirements for fast ignition. Plasma Physics and Controlled Fusion, 51(1):015016, 2009.

[33] A. J. Kemp, Y. Sentoku, and M. Tabak. Hot-electron energy coupling in ultraintense laser-matter interaction. Phys. Rev. E, 79:066406, Jun 2009.

[34] S. C. Wilks, W. L. Kruer, M. Tabak, and A. B. Langdon. Absorption of ultra-intense laser pulses. Phys. Rev. Lett., 69:1383-1386, Aug 1992.

[35] Y. Ping, A. J. Kemp, L. Divol, M. H. Key, P. K. Patel, K. U. Akli, F. N. Beg, S. Chawla, C. D. Chen, R. R. Freeman, D. Hey, D. P. Higginson, L. C. Jarrott, G. E. Kemp, A. Link, H. S. McLean, H. Sawada, R. B. Stephens, D. Turnbull, B. Westover, and S. C. Wilks. Dynamics of relativistic laser-plasma interaction on solid targets. Phys. Rev. Lett., 109:145006, Oct 2012.

[36] H Schwoerer, S Pfotenhauer, O Jäckel, K-U Amthor, B Liesfeld, W Ziegler, R Sauerbrey, KWD Ledingham, and T Esirkepov. Laser-plasma acceleration of quasimonoenergetic protons from microstructured targets. Nature, 439(7075):445-448, 2006.

[37] M. H. Key. Status of and prospects for the fast ignition inertial fusion concept. Physics of Plasmas, 14(5):055502, 2007.

[38] Xiaoling Yang, George H. Miley, Kirk A. Flippo, and Heinrich Hora. Energy enhancement for deuteron beam fast ignition of a precompressed inertial confinement fusion target. Physics of Plasmas, 18(3):032703, 2011.

[39] S. C. Wilks, A. B. Langdon, T. E. Cowan, M. Roth, M. Singh, S. Hatchett, M. H. Key, D. Pennington, A. MacKinnon, and R. A. Snavely. Energetic proton generation in ultra-intense laser-solid interactions. Physics of Plasmas, 8(2):542-549, 2001.

[40] Stephen P. Hatchett, Curtis G. Brown, Thomas E. Cowan, Eugene A. Henry, Joy S. Johnson, Michael H. Key, Jeffrey A. Koch, A. Bruce Langdon, Barbara F. Lasinski, 
Richard W. Lee, Andrew J. Mackinnon, Deanna M. Pennington, Michael D. Perry, Thomas W. Phillips, Markus Roth, T. Craig Sangster, Mike S. Singh, Richard A. Snavely, Mark A. Stoyer, Scott C. Wilks, and Kazuhito Yasuike. Electron, photon, and ion beams from the relativistic interaction of petawatt laser pulses with solid targets. Physics of Plasmas, 7(5):2076-2082, 2000.

[41] J. T. Morrison, M. Storm, E. Chowdhury, K. U. Akli, S. Feldman, C. Willis, R. L. Daskalova, T. Growden, P. Berger, T. Ditmire, L. Van Woerkom, and R. R. Freeman. Selective deuteron production using target normal sheath acceleration. Physics of Plasmas, 19(3):030707, 2012.

[42] I. Watts, M. Zepf, E. L. Clark, M. Tatarakis, K. Krushelnick, A. E. Dangor, R. Allott, R. J. Clarke, D. Neely, and P. A. Norreys. Measurements of relativistic self-phasemodulation in plasma. Phys. Rev. E, 66:036409, Sep 2002.

[43] A Upadhyay, V K Tripathi, and H C Pant. Pulse front sharpening of a laser beam in plasma. Physica Scripta, 63(4):326, 2001.

[44] A. Pukhov and J. Meyer-ter Vehn. Relativistic magnetic self-channeling of light in near-critical plasma: Three-dimensional particle-in-cell simulation. Phys. Rev. Lett., 76:3975-3978, May 1996.

[45] J. A. Stamper, K. Papadopoulos, R. N. Sudan, S. O. Dean, E. A. McLean, and J. M. Dawson. Spontaneous magnetic fields in laser-produced plasmas. Phys. Rev. Lett., 26:1012-1015, Apr 1971.

[46] R. N. Sudan. Mechanism for the generation of $10^{9} \mathrm{~g}$ magnetic fields in the interaction of ultraintense short laser pulse with an overdense plasma target. Phys. Rev. Lett., 70:3075-3078, May 1993.

[47] S. D. Baton, M. Koenig, J. Fuchs, A. Benuzzi-Mounaix, P. Guillou, B. Loupias, T. Vinci, L. Gremillet, C. Rousseaux, M. Drouin, E. Lefebvre, F. Dorchies, C. Fourment, J. J. Santos, D. Batani, A. Morace, R. Redaelli, M. Nakatsutsumi, R. Kodama, 
A. Nishida, N. Ozaki, T. Norimatsu, Y. Aglitskiy, S. Atzeni, and A. Schiavi. Inhibition of fast electron energy deposition due to preplasma filling of cone-attached targets. Physics of Plasmas, 15(4):042706, 2008.

[48] Sasi Palaniyappan, B. Manuel Hegelich, Hui-Chun Wu, Daniel Jung, Donald C. Gautier, Lin Yin, Brian J. Albright, Randall P. Johnson, Tsutomu Shimada, Samuel Letzring, Dustin T. Offermann, Jun Ren, Chengkun Huang, Rainer Hrlein, Brendan Dromey, Juan C. Fernandez, and Rahul C. Shah. Dynamics of relativistic transparency and optical shuttering in expanding overdense plasmas. Nat Phys, 8:763-769, Aug 2012. 10.1038/nphys1158.

[49] V. Yanovsky, V. Chvykov, G. Kalinchenko, P. Rousseau, T. Planchon, T. Matsuoka, A. Maksimchuk, J. Nees, G. Cheriaux, G. Mourou, and K. Krushelnick. Ultra-high intensity- 300-tw laser at $0.1 \mathrm{hz}$ repetition rate. Opt. Express, 16(3):2109-2114, Feb 2008.

[50] Robert Fedosejevs, DP Higginson, H Friesen, A Sorokovikova, LC Jarrott, A Link, GE Kemp, D Hey, Y Ping, I Bush, et al. Characterization of mev electron generation using $527 \mathrm{~nm}$ laser pulses for fast ignition. In Plasma Science (ICOPS), 2012 Abstracts IEEE International Conference on, pages 1P-134. IEEE, 2012.

[51] C. Dorrer. Characterization of a high-contrast front-end prototype for the omega ep laser facility. In Lasers and Electro-Optics (CLEO), 2011 Conference on, pages 1-2, 2011.

[52] DI Hillier, Colin Danson, Stuart Duffield, David Egan, Stephen Elsmere, Mark Girling, Ewan Harvey, Nicholas Hopps, Michael Norman, Stefan Parker, et al. Ultra high contrast from a frequency doubled cpa beamline. Appl. Optics,(submitted 2013).

[53] Patrick Poole, Chris Willis, Enam Chowdhury, Rebecca Daskalova, Linn Van Woerkom, and Rick Freeman. Petawatt-class ultra-high-peak-power laser design for the scarlet laser facility. Bulletin of the American Physical Society, 56, 2011. 
[54] Aurélie Jullien, Olivier Albert, Frédéric Burgy, Guy Hamoniaux, Jean Philippe Rousseau, Jean Paul Chambaret, Frédérika Augé Rochereau, Gilles Chériaux, Jean Etchepare, Nikolay Minkovski, and Solomon M. Saltiel. $10^{-10}$ temporal contrast for femtosecond ultraintense lasers by cross-polarized wave generation. Opt. Lett., 30(8):920-922, Apr 2005.

[55] B. Dromey, S. Kar, M. Zepf, and P. Foster. The plasma mirror - a subpicosecond optical switch for ultrahigh power lasers. Review of Scientific Instruments, 75(3):645$649,2004$.

[56] Anna Lévy, Tiberio Ceccotti, Pascal D’Oliveira, Fabrice Réau, Michel Perdrix, Fabien Quéré, Pascal Monot, Michel Bougeard, Hervé Lagadec, Philippe Martin, Jean-Paul Geindre, and Patrick Audebert. Double plasma mirror for ultrahigh temporal contrast ultraintense laser pulses. Opt. Lett., 32(3):310-312, Feb 2007.

[57] V. M. Ovchinnikov, D. W. Schumacher, M. McMahon, E. A. Chowdhury, C. D. Chen, A. Morace, and R. R. Freeman. Effects of preplasma scale length and laser intensity on the divergence of laser-generated hot electrons. Phys. Rev. Lett., 110:065007, Feb 2013.

[58] K. U. Akli, M. J. Storm, M. McMahon, S. Jiang, V. Ovchinnikov, D. W. Schumacher, R. R. Freeman, G. Dyer, and T. Ditmire. Time dependence of fast electron beam divergence in ultraintense laser-plasma interactions. Phys. Rev. E, 86:026404, Aug 2012 .

[59] T. Ma, H. Sawada, P. K. Patel, C. D. Chen, L. Divol, D. P. Higginson, A. J. Kemp, M. H. Key, D. J. Larson, S. Le Pape, A. Link, A. G. MacPhee, H. S. McLean, Y. Ping, R. B. Stephens, S. C. Wilks, and F. N. Beg. Hot electron temperature and coupling efficiency scaling with prepulse for cone-guided fast ignition. Phys. Rev. Lett., 108:115004, Mar 2012.

[60] A. G. MacPhee, L. Divol, A. J. Kemp, K. U. Akli, F. N. Beg, C. D. Chen, H. Chen, D. S. Hey, R. J. Fedosejevs, R. R. Freeman, M. Henesian, M. H. Key, S. Le Pape, 
A. Link, T. Ma, A. J. Mackinnon, V. M. Ovchinnikov, P. K. Patel, T. W. Phillips, R. B. Stephens, M. Tabak, R. Town, Y. Y. Tsui, L. D. Van Woerkom, M. S. Wei, and S. C. Wilks. Limitation on prepulse level for cone-guided fast-ignition inertial confinement fusion. Phys. Rev. Lett., 104:055002, Feb 2010.

[61] T. Ceccotti, A. Lévy, H. Popescu, F. Réau, P. D’Oliveira, P. Monot, J. P. Geindre, E. Lefebvre, and Ph. Martin. Proton acceleration with high-intensity ultrahighcontrast laser pulses. Phys. Rev. Lett., 99:185002, Oct 2007.

[62] T Ceccotti, A Lvy, F Rau, H Popescu, P Monot, E Lefebvre, and Ph Martin. Tnsa in the ultra-high contrast regime. Plasma Physics and Controlled Fusion, 50(12):124006, 2008.

[63] G Elijah Kemp, Anthony Link, Robert Fedosejevs, Richard R Freeman, Farhat N Beg, Hal Friesen, Drew P Higginson, Mike H Key, Harry S McLean, Prav Patel, et al. Specular reflectivity in high contrast relativistic laser-plasma interactions. Bulletin of the American Physical Society, 57, 2012.

[64] C. D. Chen, J. A. King, M. H. Key, K. U. Akli, F. N. Beg, H. Chen, R. R. Freeman, A. Link, A. J. Mackinnon, A. G. MacPhee, P. K. Patel, M. Porkolab, R. B. Stephens, and L. D. Van Woerkom. A bremsstrahlung spectrometer using k-edge and differential filters with image plate dosimeters. Review of Scientific Instruments, 79(10):10E305, 2008.

[65] K U Akli, P K Patel, R Van Maren, R B Stephens, M H Key, D P Higginson, B Westover, C D Chen, A J Mackinnon, T Bartal, F N Beg, S Chawla, R Fedosejevs, R R Freeman, D S Hey, G E Kemp, S LePape, A Link, T Ma, A G MacPhee, H S McLean, Y Ping, Y Y Tsui, L D Van Woerkom, M S Wei, T Yabuuchi, and S Yuspeh. A dual channel x-ray spectrometer for fast ignition research. Journal of Instrumentation, 5(07):P07008, 2010.

[66] H. Sawada, D. P. Higginson, A. Link, T. Ma, S. C. Wilks, H. S. McLean, F. Perez, 
P. K. Patel, and F. N. Beg. Characterizing the energy distribution of laser-generated relativistic electrons in cone-wire targets. Physics of Plasmas, 19(10):103108, 2012.

[67] S. D. Baton, J. J. Santos, F. Amiranoff, H. Popescu, L. Gremillet, M. Koenig, E. Martinolli, O. Guilbaud, C. Rousseaux, M. Rabec Le Gloahec, T. Hall, D. Batani, E. Perelli, F. Scianitti, and T. E. Cowan. Evidence of ultrashort electron bunches in laser-plasma interactions at relativistic intensities. Phys. Rev. Lett., 91:105001, Sep 2003.

[68] G. Malka and J. L. Miquel. Experimental confirmation of ponderomotive-force electrons produced by an ultrarelativistic laser pulse on a solid target. Phys. Rev. Lett., 77:75-78, Jul 1996.

[69] T. Yabuuchi, K. Adumi, H. Habara, R. Kodama, K. Kondo, T. Tanimoto, K. A. Tanaka, Y. Sentoku, T. Matsuoka, Z. L. Chen, M. Tampo, A. L. Lei, and K. Mima. On the behavior of ultraintense laser produced hot electrons in self-excited fields. Physics of Plasmas, 14(4):040706, 2007.

[70] V. M. Ovchinnikov, G. E. Kemp, D. W. Schumacher, R. R. Freeman, and L. D. Van Woerkom. How well do time-integrated $k_{\alpha}$ images represent hot electron spatial distributions? Physics of Plasmas, 18(7):072704, 2011.

[71] K. U. Akli, M. H. Key, H. K. Chung, S. B. Hansen, R. R. Freeman, M. H. Chen, G. Gregori, S. Hatchett, D. Hey, N. Izumi, J. King, J. Kuba, P. Norreys, A. J. Mackinnon, C. D. Murphy, R. Snavely, R. B. Stephens, C. Stoeckel, W. Theobald, and B. Zhang. Temperature sensitivity of $c u k_{\alpha}$ imaging efficiency using a spherical bragg reflecting crystal. Physics of Plasmas, 14(2):023102, 2007.

[72] B. Chrisman, Y. Sentoku, and A. J. Kemp. Intensity scaling of hot electron energy coupling in cone-guided fast ignition. Physics of Plasmas, 15(5):056309, 2008.

[73] S. Chawla, M. S. Wei, R. Mishra, K. U Akli, C. D. Chen, H. S. McLean, A. Morace, P. K. Patel, H. Sawada, Y. Sentoku, R. B. Stephens, and F. N. Beg. Effect of tar- 
get material on fast-electron transport and resistive collimation. Phys. Rev. Lett., 110:025001, Jan 2013.

[74] A. Link, R. R. Freeman, D. W. Schumacher, and L. D. Van Woerkom. Effects of target charging and ion emission on the energy spectrum of emitted electrons. Physics of Plasmas, 18(5):053107, 2011.

[75] Laurent Gremillet, Guy Bonnaud, and Francois Amiranoff. Filamented transport of laser-generated relativistic electrons penetrating a solid target. Physics of Plasmas, $9(3): 941-948,2002$.

[76] Anthony Link, KU Akli, F Beg, CD Chen, JR Davies, RR Freeman, GE Kemp, K Li, HS McLean, A Morace, et al. Particle-in-cell modeling of fast ignition experiments on the titan laser. Bulletin of the American Physical Society, 57, 2012.

[77] R. Lichters, J. Meyer ter Vehn, and A. Pukhov. Short-pulse laser harmonics from oscillating plasma surfaces driven at relativistic intensity. Physics of Plasmas, 3(9):3425$3437,1996$.

[78] D. W. Schumacher, G. E. Kemp, A. Link, R. R. Freeman, and L. D. Van Woerkom. The shaped critical surface in high intensity laser plasma interactions. Physics of Plasmas, 18(1):013102, 2011.

[79] M. Zepf, G. D. Tsakiris, G. Pretzler, I. Watts, D. M. Chambers, P. A. Norreys, U. Andiel, A. E. Dangor, K. Eidmann, C. Gahn, A. Machacek, J. S. Wark, and K. Witte. Role of the plasma scale length in the harmonic generation from solid targets. Phys. Rev. E, 58:R5253-R5256, Nov 1998.

[80] B. Dromey, D. Adams, R. Horlein, Y. Nomura, S. G. Rykovanov, D. C. Carroll, P. S. Foster, S. Kar, K. Markey, P. McKenna, D. Neely, M. Geissler, G. D. Tsakiris, and M. Zepf. Diffraction-limited performance and focusing of high harmonics from relativistic plasmas. Nat Phys, 5:146-152, Feb 2009. 10.1038/nphys1158. 
[81] S G Rykovanov, H Ruhl, J Meyer ter Vehn, R Hrlein, B Dromey, M Zepf, and G D Tsakiris. Plasma surface dynamics and smoothing in the relativistic few-cycle regime. New Journal of Physics, 13(2):023008, 2011.

[82] M. Tatarakis, A. Gopal, I. Watts, F. N. Beg, A. E. Dangor, K. Krushelnick, U. Wagner, P. A. Norreys, E. L. Clark, M. Zepf, and R. G. Evans. Measurements of ultrastrong magnetic fields during relativistic laser-plasma interactions. Physics of Plasmas, 9(5):2244-2250, 2002.

[83] A Gopal, S Minardi, M Burza, G Genoud, I Tzianaki, A Karmakar, P Gibbon, M Tatarakis, A Persson, and C-G Wahlstrm. Megagauss magnetic field generation by ultra-short pulses at relativistic intensities. Plasma Physics and Controlled Fusion, $55(3): 035002,2013$.

[84] John David Jackson. Classical electrodynamics. Wiley, New York, NY, 3rd ed. edition.

[85] P. Gibbon. Short Pulse Laser Interactions With Matter: An Introduction. Imperial College Press, 2005.

[86] Thomas A. Carlson, C.W. Nestor Jr., Neil Wasserman, and J.D. Mcdowell. Calculated ionization potentials for multiply charged ions. Atomic Data and Nuclear Data Tables, $2(0): 63-99,1970$.

[87] M. V. Ammosov, N. B. Delone, and V. P. Krainov. Tunnel ionization of complex atoms and of atomic ions in an alternating electromagnetic field. Soviet Physics JETP, 64(6):1191-1194, 1986.

[88] P.A. Sturrock. Plasma Physics: An Introduction to the Theory of Astrophysical, Geophysical and Laboratory Plasmas. Stanford-Cambridge Program. Cambridge University Press, 1994.

[89] J. C. Adam, A. Héron, and G. Laval. Dispersion and transport of energetic particles due to the interaction of intense laser pulses with overdense plasmas. Phys. Rev. Lett., 97:205006, Nov 2006. 
[90] Y. Ping, R. Shepherd, B. F. Lasinski, M. Tabak, H. Chen, H. K. Chung, K. B. Fournier, S. B. Hansen, A. Kemp, D. A. Liedahl, K. Widmann, S. C. Wilks, W. Rozmus, and M. Sherlock. Absorption of short laser pulses on solid targets in the ultrarelativistic regime. Phys. Rev. Lett., 100:085004, Feb 2008.

[91] W.L. Kruer. The Physics of Laser Plasma Interactions. Frontiers in Physics. Westview Press, 2003.

[92] B. M. Penetrante, J. N. Bardsley, W M. Wood, C. W Siders, and M. C. Downer. Ionization-induced frequency shifts in intense femtosecond laser pulses. J. Opt. Soc. Am. B, 9(11):2032-2040, Nov 1992.

[93] PASQUALE Mazzotta, GABRIELE Mazzitelli, S Colafrancesco, and N Vittorio. Ionization balance for optically thin plasmas: Rate coefficients for all atoms and ions of the elements h to ni. Astronomy and Astrophysics Supplement Series, 133(3):403-409, 1998.

[94] V. I. Eremin, A. V. Korzhimanov, and A. V. Kim. Relativistic self-induced transparency effect during ultraintense laser interaction with overdense plasmas: Why it occurs and its use for ultrashort electron bunch generation. Physics of Plasmas, 17(4):043102, 2010.

[95] Claire Ellen Max, Jonathan Arons, and A. Bruce Langdon. Self-modulation and self-focusing of electromagnetic waves in plasmas. Phys. Rev. Lett., 33:209-212, Jul 1974 .

[96] AG Litvak. Finite-amplitude wave beams in a magnetoactive plasma. Sov. Phys. JETP, 30(344):166, 1970.

[97] RR Freeman, D Batani, S Baton, M Key, and R Stephens. The generation and transport of large currents in dense materials: the physics of electron transport relative to fast ignition. Fusion science and technology, 49(3):297, 2006. 
[98] Yasuhiko Sentoku, Kunioki Mima, Shin ichi Kojima, and Hartmut Ruhl. Magnetic instability by the relativistic laser pulses in overdense plasmas. Physics of Plasmas, $7(2): 689-695,2000$.

[99] Erich S. Weibel. Spontaneously growing transverse waves in a plasma due to an anisotropic velocity distribution. Phys. Rev. Lett., 2:83-84, Feb 1959.

[100] F. Brunel. Not-so-resonant, resonant absorption. Phys. Rev. Lett., 59:52-55, Jul 1987.

[101] W. L. Kruer and Kent Estabrook. J x b heating by very intense laser light. Physics of Fluids, 28(1):430-432, 1985.

[102] J. May, J. Tonge, F. Fiuza, R. A. Fonseca, L. O. Silva, C. Ren, and W. B. Mori. Mechanism of generating fast electrons by an intense laser at a steep overdense interface. Phys. Rev. E, 84:025401, Aug 2011.

[103] Charles K Birdsall and A Bruce Langdon. Plasma physics via computer simulation. CRC Press, 2005.

[104] D. R. Welch, D. V. Rose, M. E. Cuneo, R. B. Campbell, and T. A. Mehlhorn. Integrated simulation of the generation and transport of proton beams from laser-target interaction. Physics of Plasmas, 13(6):063105, 2006.

[105] D.R. Welch, D.V. Rose, R.E. Clark, T.C. Genoni, and T.P. Hughes. Implementation of an non-iterative implicit electromagnetic field solver for dense plasma simulation. Computer Physics Communications, 164(13):183 - 188, 2004. ¡ce:title¿Proceedings of the 18th International Conferene on the Numerical Simulation of Plasmasi/ce:title

[106] LLNL. Jupiter laser facility: Titan laser, February 2013. http://jlf.llnl.gov/.

[107] General Atomics. General atomics, February 2013. http://www.ga.com/.

[108] Rick Trebino and Daniel J. Kane. Using phase retrieval to measure the intensity and phase of ultrashort pulses: frequency-resolved optical gating. J. Opt. Soc. Am. A, 10(5):1101-1111, May 1993. 
[109] Daniel J. Kane and Rick Trebino. Single-shot measurement of the intensity and phase of an arbitrary ultrashort pulse by using frequency-resolved optical gating. Opt. Lett., 18(10):823-825, May 1993.

[110] Henry Tiedje. private communication, 2012.

[111] Ocean Optics. Ocean optics hr4000 spectrometer, February 2013. http://www. oceanoptics. com/Products/hr4000. asp.

[112] Coherent: Molectron Detector Incorporated. J25lp-xa/j50lp-xa: Large area amplified detectors, January 2003. http://www. coherent. com/Downloads/J2550LPxA. pdf.

[113] Apogee. High performance cooled ccd camera system alta u47, January 2010. http: //WWW.ccd.com/pdf/U47.pdf.

[114] Shaun Kerr. private communication, 2011.

[115] Robert Fedosejevs. private communication, 2012.

[116] R. Ramis, J. Meyer ter Vehn, and J. Ramí?rez. Multi2d a computer code for two-dimensional radiation hydrodynamics. Computer Physics Communications, 180(6):977 - 994, 2009.

[117] M. Raynaud, J. Kupersztych, C. Riconda, J. C. Adam, and A. Heron. Strongly enhanced laser absorption and electron acceleration via resonant excitation of surface plasma waves. Physics of Plasmas, 14(9):092702, 2007.

[118] A. Macchi, F. Cornolti, F. Pegoraro, T. V. Liseikina, H. Ruhl, and V. A. Vshivkov. Surface oscillations in overdense plasmas irradiated by ultrashort laser pulses. Phys. Rev. Lett., 87:205004, Oct 2001.

[119] Hartmut Ruhl, Andrea Macchi, Peter Mulser, Fulvio Cornolti, and Steffen Hain. Collective dynamics and enhancement of absorption in deformed targets. Phys. Rev. Lett., 82:2095-2098, Mar 1999. 
[120] A. Bigongiari, M. Raynaud, C. Riconda, A. Heron, and A. Macchi. Efficient laseroverdense plasma coupling via surface plasma waves and steady magnetic field generation. Physics of Plasmas, 18(10):102701, 2011.

[121] Guang yue Hu, An le Lei, Jing wei Wang, Lin gen Huang, Wen tao Wang, Xin Wang, Yi Xu, Bai fei Shen, Jian sheng Liu, Wei Yu, Ru xin Li, and Zhi zhan Xu. Enhanced surface acceleration of fast electrons by using subwavelength grating targets. Physics of Plasmas, 17(8):083102, 2010.

[122] O Klimo, J Psikal, J Limpouch, J Proska, F Novotny, T Ceccotti, V Floquet, and S Kawata. Short pulse laser interaction with micro-structured targets: simulations of laser absorption and ion acceleration. New Journal of Physics, 13(5):053028, 2011.

[123] G. Binnig, C. F. Quate, and Ch. Gerber. Atomic force microscope. Phys. Rev. Lett., 56:930-933, Mar 1986.

[124] Goodfellow. Goodfellow, February 2013. http://www.goodfellowusa.com/.

[125] Labsphere. Labsphere, February 2013. http://www.labsphere.com/default.aspx.

[126] Labsphere. Technical guide: Reflectance materials and coatings, February 2013. http://www.labsphere.com/uploads/technical-guides/ a-guide-to-reflectance-materials-and-coatings.pdf.

[127] Wikipedia. Lambert's cosine law, June 2013. https://en.wikipedia.org/wiki/ Lambert\%27s_cosine_law.

[128] Andor. Andor dv434 ccd, February 2013. ftp://ftp.aerodyne.com/users/Jones/ NASA/DV434.pdf.

[129] Anthony J. Link. private communication, 2012.

[130] Ocean Optics. Ocean optics usb2000 mini spectrometer, February 2013. http://www. oceanoptics.com/Products/usb2000. asp. 
[131] Schott. Kg3 data sheet, February 2013. http://www.schott.com/advanced_optics/ english/download/schott_shortpass_kg3_2008_eng.pdf.

[132] L. Van Woerkom, K. U. Akli, T. Bartal, F. N. Beg, S. Chawla, C. D. Chen, E. Chowdhury, R. R. Freeman, D. Hey, M. H. Key, J. A. King, A. Link, T. Ma, A. J. MacKinnon, A. G. MacPhee, D. Offermann, V. Ovchinnikov, P. K. Patel, D. W. Schumacher, R. B. Stephens, and Y. Y. Tsui. Fast electron generation in cones with ultraintense laser pulses. Physics of Plasmas, 15(5):056304, 2008.

[133] V. M. Ovchinnikov, D. W. Schumacher, G. E. Kemp, A. G. Krygier, L. D. Van Woerkom, K. U. Akli, R. R. Freeman, R. B. Stephens, and A. Link. Using timeintegrated $k_{\alpha}$ images to study refluxing and the extent of pre-plasmas in intense laser-plasma experiment. Physics of Plasmas, 18(11):112702, 2011.

[134] Erik Lefebvre and Guy Bonnaud. Transparency/opacity of a solid target illuminated by an ultrahigh-intensity laser pulse. Phys. Rev. Lett., 74:2002-2005, Mar 1995.

[135] M. Borghesi, A. Giulietti, D. Giulietti, L. A. Gizzi, A. Macchi, and O. Willi. Characterization of laser plasmas for interaction studies: Progress in time-resolved density mapping. Phys. Rev. E, 54:6769-6773, Dec 1996.

[136] L. A. Gizzi, D. Giulietti, A. Giulietti, T. Afshar-Rad, V. Biancalana, P. Chessa, C. Danson, E. Schifano, S. M. Viana, and O. Willi. Characterization of laser plasmas for interaction studies. Phys. Rev. E, 49:5628-5643, Jun 1994.

[137] A. S. Pirozhkov, I. W. Choi, J. H. Sung, S. K. Lee, T. J. Yu, T. M. Jeong, I J. Kim, N. Hafz, C. M. Kim, K. H. Pae, Y.-C. Noh, D.-K. Ko, J. Lee, A. P. L. Robinson, P. Foster, S. Hawkes, M. Streeter, C. Spindloe, P. McKenna, D. C. Carroll, C.-G. Wahlstrom, M. Zepf, D. Adams, B. Dromey, K. Markey, S. Kar, Y. T. Li, M. H. Xu, H. Nagatomo, M. Mori, A. Yogo, H. Kiriyama, K. Ogura, A. Sagisaka, S. Orimo, M. Nishiuchi, H. Sugiyama, T. Zh. Esirkepov, H. Okada, S. Kondo, S. Kanazawa, Y. Nakai, A. Akutsu, T. Motomura, M. Tanoue, T. Shimomura, M. Ikegami, I. Daito, 
M. Kando, T. Kameshima, P. Bolton, S. V. Bulanov, H. Daido, and D. Neely. Diagnostic of laser contrast using target reflectivity. Applied Physics Letters, 94(24):241102, 2009.

[138] D.R. Welch, D.V. Rose, B.V. Oliver, and R.E. Clark. Simulation techniques for heavy ion fusion chamber transport. Nuclear Instruments and Methods in Physics Research Section A: Accelerators, Spectrometers, Detectors and Associated Equipment, 464(13):134 - 139, 2001. 13th International Symposium on Heavy Ion Inertial Fusion.

[139] Michael E. Jones, Don S. Lemons, Rodney J. Mason, Vincent A. Thomas, and Dan Winske. A grid-based coulomb collision model for $\{\mathrm{PIC}\}$ codes. Journal of Computational Physics, 123(1):169 - 181, 1996.

[140] A.E. Siegman. Lasers. University Science Books, 1986.

[141] J.C. Diels and W. Rudolph. Ultrashort Laser Pulse Phenomena. Optics and photonics. Elsevier Science, 2006.

[142] J. Meyer ter Vehn and Z. M. Sheng. On electron acceleration by intense laser pulses in the presence of a stochastic field. Physics of Plasmas, 6(3):641-644, 1999.

[143] Mitsumori Tanimoto, Susumu Kato, Eisuke Miura, Naoaki Saito, Kazuyoshi Koyama, and James K. Koga. Direct electron acceleration by stochastic laser fields in the presence of self-generated magnetic fields. Phys. Rev. E, 68:026401, Aug 2003.

[144] Tatsufumi Nakamura, Susumu Kato, Mitsumori Tamimoto, and Tomokazu Kato. Stochastic acceleration by intense laser fields. Physics of Plasmas, 9(5):1801-1805, 2002.

[145] A. G. Krygier. On the Origin of Super-Hot Electrons in Intense Laser-Plasma Interactions. PhD thesis, The Ohio State University, May 2013.

[146] G. E. Kemp, A. Link, Y. Ping, D. W. Schumacher, R. R. Freeman, and P. K. Patel. 
Coupling of laser energy into hot-electrons in high-contrast relativistic laser-plasma interactions. Physics of Plasmas, 20(3):033104, 2013.

[147] D. Margarone, O. Klimo, I. J. Kim, J. Prokůpek, J. Limpouch, T. M. Jeong, T. Mocek, J. Pšikal, H. T. Kim, J. Proška, K. H Nam, L. Štolcová, I. W. Choi, S. K. Lee, J. H. Sung, T. J. Yu, and G. Korn. Laser-driven proton acceleration enhancement by nanostructured foils. Phys. Rev. Lett., 109:234801, Dec 2012.

[148] A. J. Kemp and L. Divol. Interaction physics of multipicosecond petawatt laser pulses with overdense plasma. Phys. Rev. Lett., 109:195005, Nov 2012.

[149] A. Gopal, M. Tatarakis, F. N. Beg, E. L. Clark, A. E. Dangor, R. G. Evans, P. A. Norreys, M. S. Wei, M. Zepf, and K. Krushelnick. Temporally and spatially resolved measurements of multi-megagauss magnetic fields in high intensity laser-produced plasmas. Physics of Plasmas, 15(12):122701, 2008.

[150] R. Trebino. Frequency-Resolved Optical Gating: The Measurement of Ultrashort Laser Pulses. Kluwer Academic Pub, 2000.

[151] An-Chun Tien, Steve Kane, Jeff Squier, Bern Kohler, and Kent Wilson. Geometrical distortions and correction algorithm in single-shot pulse measurements: application to frequency-resolved optical gating. J. Opt. Soc. Am. B, 13(6):1160-1165, Jun 1996.

[152] Daniel J. Kane. Principal components generalized projections: a review. J. Opt. Soc. Am. B, 25(6):A120-A132, Jun 2008.

[153] D.J. Kane. Real-time measurement of ultrashort laser pulses using principal component generalized projections. Selected Topics in Quantum Electronics, IEEE Journal of, 4(2):278-284, 1998.

[154] K.W. DeLong, D.N. Fittinghoff, and R. Trebino. Practical issues in ultrashort-laserpulse measurement using frequency-resolved optical gating. Quantum Electronics, IEEE Journal of, 32(7):1253-1264, jul 1996. 
[155] Ziyang Wang, Erik Zeek, Rick Trebino, and Paul Kvam. Determining error bars in measurements of ultrashort laser pulses. J. Opt. Soc. Am. B, 20(11):2400-2405, Nov 2003.

[156] B.I. Cohen, A.J. Kemp, and L. Divol. Simulation of laser-plasma interactions and fast-electron transport in inhomogeneous plasma. Journal of Computational Physics, 229(12):4591 - 4612, 2010.

[157] M. M. Marinak, S. W. Haan, T. R. Dittrich, R. E. Tipton, and G. B. Zimmerman. A comparison of three-dimensional multimode hydrodynamic instability growth on various national ignition facility capsule designs with [small-caps hydra] simulations. Physics of Plasmas, 5(4):1125-1132, 1998.

[158] M.H. et al Key. Proceedings of the Seventh Conference on Inertial Fusion Sciences and Applications (IFSA 2011). 


\section{Appendix A \\ Laser Diagnostic Table Layout}

Figure A.1 contains the specific layout of the laser diagnostic table.

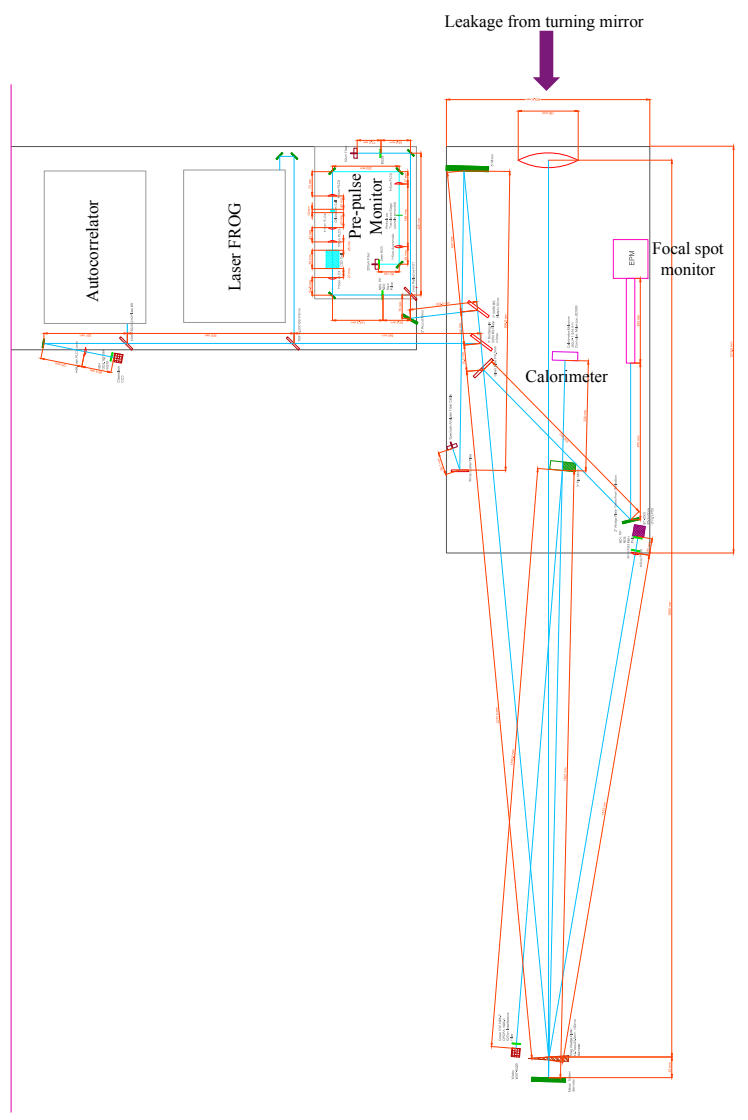

Figure A.1: (Color) Laser diagnostic table layout for the experiment, courtesy of H. Tiedje [110]. 


\section{Appendix B PRE-PUlSE MONITOR}

Figure B.1 contains the specific layout of the laser diagnostic table.

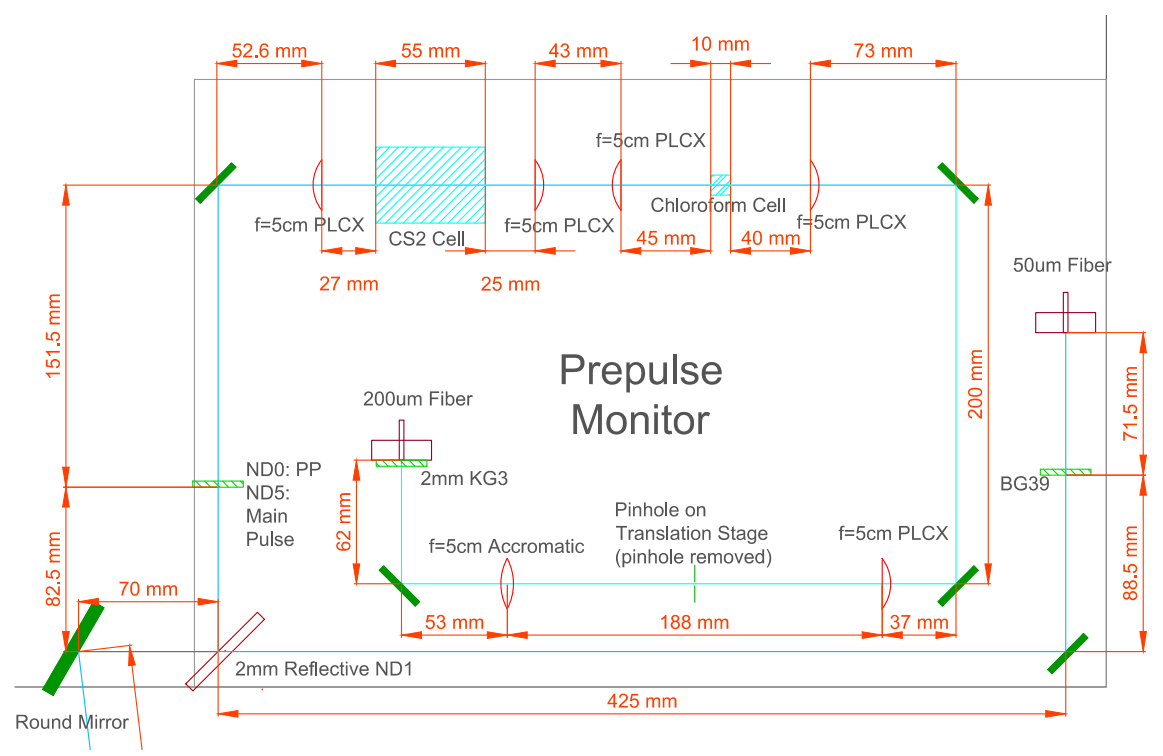

Figure B.1: (Color) Layout of the pre-pulse monitor used in the experiment, courtesy of H. Tiedje [110]. 


\section{Appendix $\mathrm{C}$ SHORT PULSE MEASUREMENT: Frequency Resolved Optical GATING}

Most of the diagnostics discussed in this thesis are fairly common or at least straightforward, perhaps with the exception of the temporal short pulse characterization. As a significant fraction of the experimental results concern this diagnostic, it is worth some time to briefly go through the theory behind the technique.

How can we measure the temporal evolution of a laser pulse that is a few mere picoseconds from beginning to end with 10 s of femtoseconds resolution? Currently, the only technique that can provide this kind of resolution requires the use of another short pulse. Using an optical gating approach, Rick Trebino developed a technique that uses another ultrashort pulse to simultaneously measure both pulses, known as Frequency Resolved Optical Gating or FROG [108]. In principle, a FROG measurement is a correlation of the two pulses that has been resolved in frequency with an imaging spectrometer. The result is a $2 \mathrm{D}$ intensity distribution that is a function of both frequency and relative temporal delay between the two pulses known as a spectrogram. Technically speaking, this is the Fourier-transform of the correlation between the electric fields of the desired incident pulse $E(t)$ and the gating pulse $G(t)$ at each relative delay value creating the spectrograph $\left(|S(\omega, \tau)|^{2}\right)$ as shown in Equation C.1. Using an iterative phase retrieval algorithm, the full temporal pulse information (both intensity and phase) of both the incident and gating pulses, in theory, can be uniquely retrieved from the real valued intensity spectrogram 
[109].

$$
I(\omega, \tau)=|S(\omega, \tau)|^{2}=\left|\int_{-\infty}^{\infty} E(t) G(t-\tau) e^{-\imath \omega t} \mathrm{~d} t\right|^{2}
$$

As you might guess, there are many configurations in which FROG traces can be generated but, in principle, they all operate under the same theory. Typically, only one pulse measurement is desired so a self-gating technique is employed. Instead of the blind FROG configuration where neither the incident or gating pulses are known, the incident pulse is split in two using a beam splitter and used to measure itself with an autocorrelation geometry. This simplifies the phase retrieval algorithm by a priori placing a constraint on the gating pulse $G(t)$ by having it as a function of the incident pulse $E(t)$. There are several popular configurations using this self-gating approach, some of which are shown in Table C.1 [150]. Each configuration has it advantages and disadvantages. If signal levels are lim-

\begin{tabular}{ccccc}
\hline \hline Geometry & Nonlinearity & Effect & $G(t)$ & Sensitivity $[\mu J]$ \\
\hline SHG & $\chi^{(2)}$ & Second harmonic generation & $E(t)$ & 0.01 \\
THG & $\chi^{(3)}$ & Third harmonic generation & $E(t)^{2}$ & 0.03 \\
PG & $\chi^{(3)}$ & Optical Kerr effect & $|E(t)|^{2}$ & 1 \\
\hline \hline
\end{tabular}

Table C.1: Gating pulses for popular FROG geometries [150].

ited, then the second harmonic generation (SHG) FROG geometry is best as the conversion efficiency of this process (using KDP or BBO) is typically the highest of all other sumfrequency generation (SFG) processes, typically requiring only $n J$ 's of energy [150]. Also, it is easy to separate out the signal from the unconverted light as the FROG signal is a different color than the incident pulses. Although it will provide the highest signal levels (as well as good signal-to-noise levels), the resultant trace is symmetric in time creating some ambiguity in the retrieval (which can be removed but only with additional measurements). Also, since efficient SHG requires phase matching, measurements of broad bandwidth pulses can be complicated. The polarization-gated (PG) FROG geometry not only removes the time directionality ambiguity (being asymmetric in time), the incident and gating pulses 
are also automatically phase matched (since it is based on induced birefringence from the optical Kerr effect and only uses one color of light) making it readily adaptable to different color pulses. However, signal-to-noise levels can be poor as the configuration relies on the extinction ratio of polarizers, often leading to some elevated background levels referred to here as 'leakage', and since it is third order the energy and intensity detection threshold is higher than lower order configurations.

In principle, a FROG trace can be constructed by scanning through the relative delay $\tau$ between the two pulses and obtaining the spectrum at each value. This multi-shot configuration is not particularly practical in for our purposes for several reasons: (1) there are so few actual full energy data shots and (2) shot-to-shot fluctuations are significant enough to produce an un-retrievable trace. Alternatively, we chose a rep-rated, single-shot configuration which is obtained by crossing the incident and gating pulses at some angle relative to each other in the non-linear medium. In particular, I will limit further discussion to only the single-shot PG FROG geometry as that was the only configuration used in these experiments.

\section{C.1 Single-shot, polarization-gated FROG}

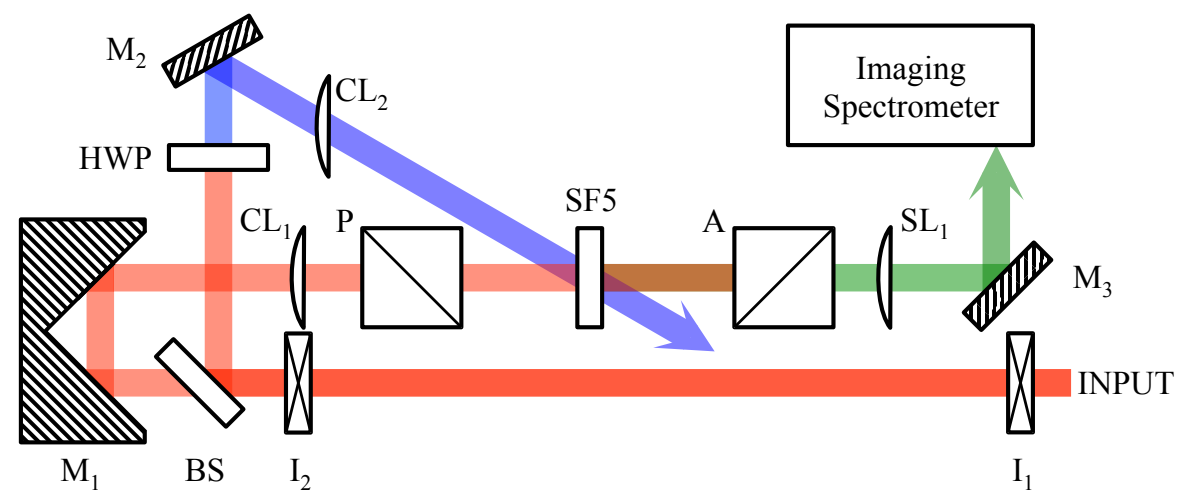

Figure C.1: (Color) Configuration for the single-shot PG FROG. $I=$ iris, $B S=$ beam splitter, $M=$ mirror, $H W P=$ half wave plate, $C L=$ cylindrical lens, $P=$ polarizer, $A=$ analyzer, $S F 5=$ nonlinear medium and $S L=$ spherical lens. $r e d=$ horizontal polarization, blue $=45$ degree polarization and green $=$ vertical polarization 
The geometry of a single-shot PG FROG is shown in Figure C.1. The incident pulse is first sent through an input polarizer (passing only the horizontally polarized light, shown in red) and then aligned to the two input irises $\left(I_{1}\right.$ and $\left.I_{2}\right)$. The pulse is then split equally using a beam splitter $(B S)$ where the transmitted light is sent to a delay arm $M_{1}$ and the polarization of the reflected light is rotated $45 \mathrm{deg}$ with respect to the incident pulse (blue). Both pulses are then focused down to a line onto the nonlinear medium $\left(N-S F 5\right.$ Schott ${ }^{\circledR}$ glass in this case, a dense flint glass with high $\chi^{(3)}$ nonlinearity) using cylindrical lenses $\left(C L_{1}\right.$ and $\left.C L_{2}\right)$ and overlapped in space. The transmitted pulse is incident normally on the medium and the gating pulse crosses at some angle. Induced birefringence (from the optical Kerr effect) in the non-linear medium created by the gating pulse rotates the polarization of the transmitted pulse but only when the gating pulse is present, creating the desired self-gated autocorrelation signal (green). Using an analyzer (simply a polarizer that passes polarization $90 \mathrm{deg}$ with respect to the transmitted pulse polarization), we can separate the autocorrelation signal with rotated polarization from the unrotated transmitted pulse. The autocorrelation signal is then sent to an imaging spectrometer, where a grating provides spectral resolution along one dimension and spatial resolution along the other. Since the pulses crossed at an angle in the medium, we can remap this spatial dimension to relative delay between the two pulses.

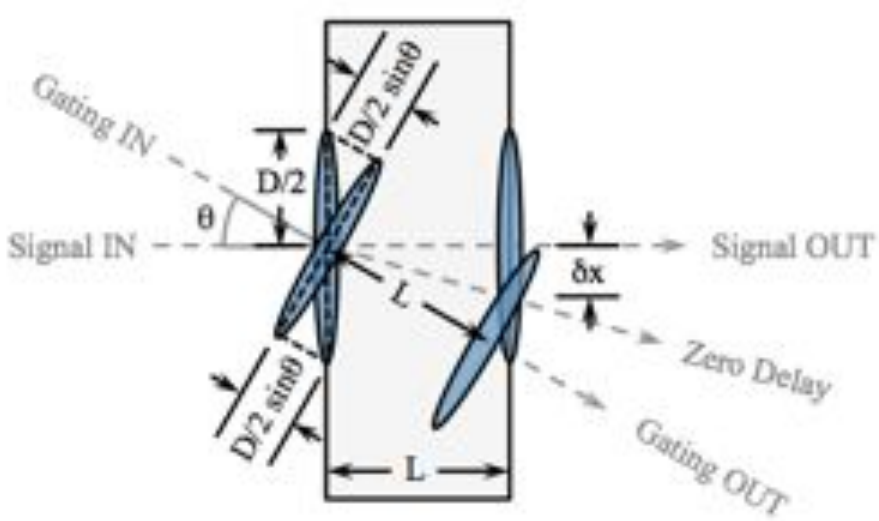

Figure C.2: Geometry for asymmetric mixing in the PG FROG 
Figure C.2 illustrates how the mixing angle in the nonlinear medium can be used to remap the spatial dimension into relative temporal delay. For this simple picture, let's assume that each pulse has a uniform spatial profile with some fixed diameter $D$, where the incident pulse is incident normally on the medium and the gating pulse crosses at an angle $\theta_{1}$ outside the medium. Since each pulse is traveling at the speed of light in the medium $c / \eta$ where $\eta$ is the refractive index, the theoretical maximum delay window in this crossedbeam configuration is $\Delta \tau=\left(\eta D / \sin \theta_{2}\right) / c$ where $\theta_{2}=\arcsin \left(\sin \left(\theta_{1}\right) / n\right)$ is the angle the two pulses cross at in the medium, due to refraction, as given by Snell's law. Clearly, the relative delay $\tau$ varies linearly as a function of overlap position $x$ is given by Equation C.2. However, some geometrical issues can arise from this configuration.

$$
\tau(x)=\frac{n x}{c} \sin \theta_{2}=\frac{x}{c} \sin \theta_{1}
$$

Since the autocorrelation signal is created parallel to the transmitted pulse and not at the bisector angle between the transmitted and gating pulses, the zero-delay axis at the entrance of the nonlinear medium has drifted in space by the time it exits the medium. The spatial drift in the zero-delay axis blurs the signal, limiting the temporal resolution of the measurement. The thickness $L$ of the non-linear medium and mixing angle determine the severity of this effect. From simple geometrical arguments, the amount of spatial drift in the zero-delay axis, $\delta x$, is $L \tan \left(\theta_{2} / 2\right)$. When mapped to delay space with Equation C.2, the PG FROG configuration has a limited theoretical temporal resolution as given by Equation C.3. As you can see, thinner nonlinear medium and more smaller angles reduce the geometrical blurring effecte and give a higher theoretical resolution. However, depending on the application, large mixing angles and thicker nonlinear media may be the only way to achieve a large enough temporal window for longer pulse measurements (see Appendix D for more information on longer pulse measurements). If found to be too severe, this effect of temporal blurring can be corrected [151].

$$
\delta \tau=\frac{n L}{c}(1-\cos \theta)
$$

Another concern for FROGs is spatial non-uniformity in the pulse intensity profile, in 
particular, after being focused down to a line with the cylindrical lenses. If the spatial profile is known, it theoretically can be deconvolved from the measurement. If there is sufficient energy in the pulse, a spatial filter can be used to filter out high frequency spatial modes and produce a much smoother line focus.

\section{C.2 Pulse retrieval}

Now that we have an experimentally obtained, real valued, FROG intensity spectrogram, an iterative phase retrieval algorithm is used to extract the complex electric field (both intensity and phase) that created the trace. The algorithm [152], known as the principle components generalized projections algorithm (PCGPA) is outlined in Figure C.3. It starts with an initial guess for the incident electric field: this is typically a gaussian temporal envelope with random phase. This initial guess is then used to generate a FROG trace as a function of angular frequency $\omega$ and delay $\tau$. Next, the intensity of the generated FROG trace is replaced by the experimental measurement while the phase from the guess is retained. This modified trace isn't an actual FROG trace, but rather a linear combination

of multiple incident and gating pulses with varying eigenvalue strengths. A new FROG trace is created using only incident/gating pulse pair with the largest eigenvalue. The current complex electric field is used as the input for the next iteration, where the procedure repeats until the algorithm converges or stagnates. The FROG error $\epsilon_{F R O G}$ is defined to be the RMS difference between the reconstructed and experimental FROG intensity spectrograms, and is given by Equation C.4.

$$
\epsilon_{F R O G}=\sqrt{\frac{1}{N^{2}} \sum_{i=1}^{N} \sum_{j=1}^{N}\left[I_{C A L C}\left(\omega_{i}, \tau_{j}\right)-\alpha I_{F R O G}\left(\omega_{i}, \tau_{j}\right)\right]^{2}}
$$

The only piece of the puzzle that's missing is how to extract the principle eigenvectors from a given complex FROG trace. Fortunately, there exists a convenient and fast algorithm for converting a incident and gating pulse into a FROG trace and, most importantly, it is a reversible procedure [152]. Let's start with a discrete $N \times N$ FROG image in $\omega / \tau$ space. The incident $E$ and gating $G$ pulses that created that trace are discrete functions of time 


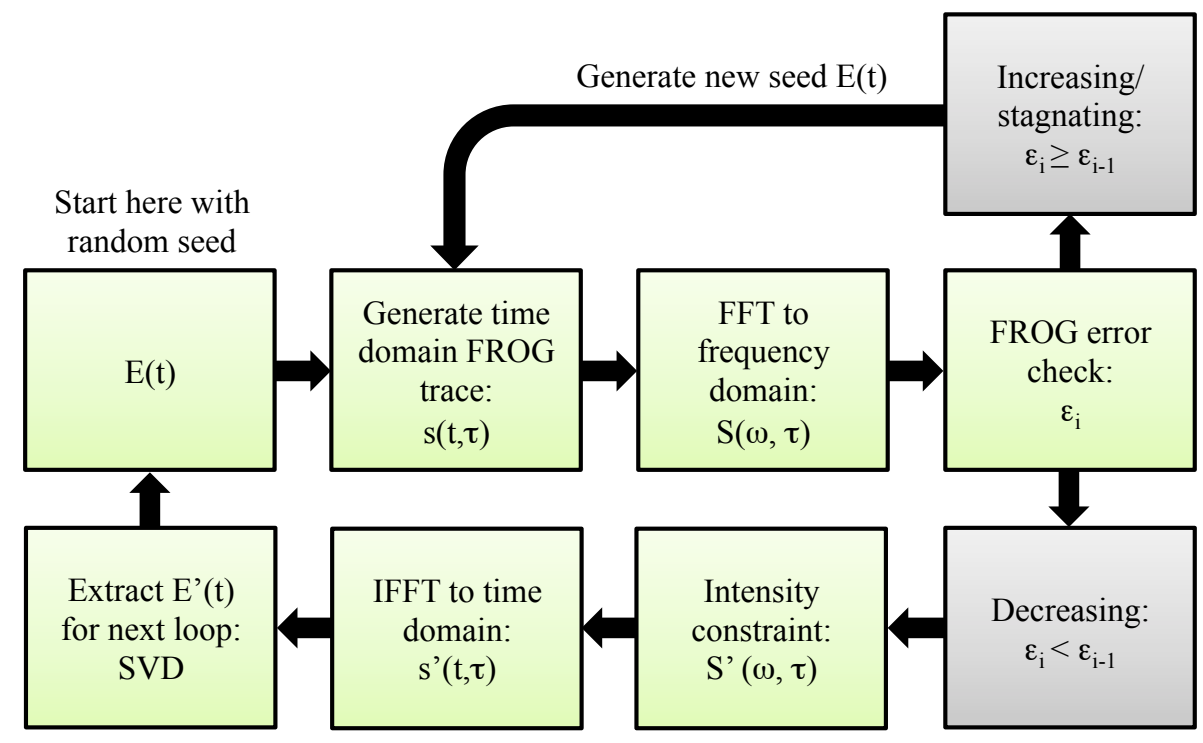

Figure C.3: PCGP algorithm for FROG measurement pulse retrieval

given by Equation C.5 where $E_{i} / G_{i}$ are the incident/gating field values at time $t_{i}$. We first generate the square outer product form $O$ of these two vectors, defined by $\left(E_{\text {probe }}\right)^{T}\left(G_{\text {gate }}\right)$ and shown in Equation C.6. By a simple row manipulation (circularly shifting each row $m \mid m \in 1: N$ by $m-1$ columns to the left) we can obtain a form that looks like Equation C.7.

$$
\begin{gathered}
E=\left[E_{1}, E_{2}, E_{3}, \ldots, E_{N}\right] \\
G=\left[G_{1}, G_{2} G_{3}, \ldots, G_{N}\right] \\
O=(E)^{T}(G)=\left[\begin{array}{cccccc}
E_{1} G_{1} & E_{1} G_{2} & E_{1} G_{3} & E_{1} G_{4} & \ldots & E_{1} G_{N} \\
E_{2} G_{1} & E_{2} G_{2} & E_{2} G_{3} & E_{2} G_{4} & \ldots & E_{2} G_{N} \\
E_{3} G_{1} & E_{3} G_{2} & E_{3} G_{3} & E_{3} G_{4} & \ldots & E_{3} G_{N} \\
E_{4} G_{1} & E_{4} G_{2} & E_{4} G_{3} & E_{4} G_{4} & \ldots & E_{4} G_{N} \\
\cdot & . & . & . & \ldots & \cdot \\
E_{N} G_{1} & E_{N} G_{2} & E_{N} G_{3} & E_{N} G_{4} & \ldots & E_{N} G_{N}
\end{array}\right]
\end{gathered}
$$




$$
\begin{gathered}
{\left[\begin{array}{cccccc}
E_{1} G_{1} & E_{1} G_{2} & E_{1} G_{3} & E_{1} G_{4} & \ldots & E_{1} G_{N} \\
E_{2} G_{2} & E_{2} G_{3} & E_{2} G_{4} & E_{2} G_{5} & \ldots & E_{2} G_{1} \\
E_{3} G_{3} & E_{3} G_{4} & E_{3} G_{5} & E_{3} G_{6} & \ldots & E_{3} G_{2} \\
E_{4} G_{4} & E_{4} G_{5} & E_{4} G_{6} & E_{4} G_{7} & \ldots & E_{4} G_{3} \\
\cdot & \cdot & . & . & \ldots & . \\
E_{N} G_{N} & E_{N} G_{N+1} & E_{N} G_{N+2} & E_{N} G_{N+3} & \ldots & E_{N} G_{N-2}
\end{array}\right]} \\
{\left[\begin{array}{cccccc}
\tau=0 & \tau=-\delta t & \tau=-2 \delta t & \tau=-3 \delta t & \ldots & \tau=\delta t
\end{array}\right]}
\end{gathered}
$$

In this form (Equation C.7) each column represents a unique delay between the signal and gating pulses. Using column manipulation, we can re-arrange these columns to be increasing in delay from left to right. After taking a Fourier transform along the columns, we obtain the complex FROG trace where the real valued spectrogram is obtained by the square of the modulus of this trace. Now, we have a one-to-one mapping from a FROG trace to its outer product from, which has a unique eigenvector pair solution.

With the application of the intensity constraint, however, we create a trace that no longer has a unique eigenvalue i.e. in general there are several incident/gating pulse eigenvectors that contribute to the newly created trace. Many methods for obtaining the predominantly contributing pair to the trace exist such as the vanilla algorithm, power method or singular value decomposition (SVD). The vanilla algorithm, as the name implies, is the simplest technique for extracting the electric field from a given FROG trace. It simply obtains a guess for the electric field by integrating the outer product form along the delay axis. Although this algorithm is often insufficient for convergence [108], it provides a good initial guess for the algorithm. The power method closely approximates the principle eigenvector by multiplying the current guess by $\left(O^{T} O\right)^{p}$ where $p$ is a large integer [152]. SVD algorithms are typically much more computationally expensive than either the vanilla or power algorithms, but all of the eigenvalue/eigenvector pairs can be extracted and, in theory, be used for error analysis [152].

The SVD algorithm massages the outer product form of the trace (Equation C.6) into 
the product of three matrices as $O=U \times W \times V^{T}$ where the columns of $U$ are the incident pulse eigenvectors, the rows of $V^{T}$ are the gating pulse eigenvectors and the diagonal values of $W$ (the only non-zero values) are the eigenvalues for each eigenvector pair. By choosing the eigenvalue pair of signal and gating pulses that dominate the outer product form of the trace in each iteration, we ensure that we minimize the FROG error in each iteration.

With any of these algorithms, a new guess for the intensity and phase is obtained and the iterations stop whenever the FROG error is sufficiently small to constrain the problem. However, any given algorithm is prone to stagnate at a local FROG error minima (can be better or worse for some geometries/algorithms) before reaching an acceptable FROG error so it is often beneficial to alternate between algorithms when stagnation occurs to reach the global minimum FROG error. For the PG FROG in particular, more robust algorithms only apply the intensity constraint on every other iteration [153]. Another useful option is to modify the current stagnated solution and restarting the SVD retrieval algorithm. Some of the more useful techniques I have found involve slight temporal smoothing of the intensity/phase or adding some small random noise to the intensity/phase.

\section{C.3 Preparing the data for retrieval}

The PG FROGs used in this experiment used N-SF5 Schott glass as the nonlinear medium. The Sellmeier equation for the index of refraction of N-SF5 is given by Equation C.8 where the index of refraction for the $527 \mathrm{~nm}$ pulses we used was 1.68 . The pulses had a $1 \mathrm{~cm}$ diameter, crossed at $30 \mathrm{deg}$ outside the medium (17.3 deg inside) resulting in a temporal window of $\Delta \tau=16.7 \mathrm{ps}$. The Schott Glass ${ }^{\circledR}$ was $500 \mu \mathrm{m}$ thick, resulting in a temporal blurring of $\delta \tau=127 \mathrm{fs}$. The detection threshold for a $500 \mathrm{fs}$ pulse was found to be about $100 \mu J$.

$$
n_{N-S F 5}(\lambda[\mu m])=\sqrt{1+\frac{1.52481889 \lambda^{2}}{\lambda^{2}-0.011254756}+\frac{0.187085527 \lambda^{2}}{\lambda^{2}-0.0588995392}+\frac{1.42729015 \lambda^{2}}{\lambda^{2}-129.141675}}
$$

As with any other experimental measurement, FROG traces obtained in the lab will also have deviations from the idealized FROG measurement described previously. These 
deviations arise from poor signal-to-noise levels, geometrical smearing, non-uniform spatial intensity distribution, leakage from the polarizer/analyzer setup, quantum fluctuations and non-linear response in the CCD detector, (etc...). Depending on the severity of each of these issues, there is a strong chance that create an physically un-retrievable FROG trace according to the description above. Great care must be taken in obtaining these traces to retrieve a meaningful pulse profile.

Two types of noise can arise: (1) multiplicative noise which is independent of intensity and (2) pixel-to-pixel gain variation, both of which can lead to noisy looking retrieved pulses [150]. Through post-processing, these sources can be removed by a mean subtraction of the background levels along with a high frequency filtering of the $2 D$ trace.

FROG traces here were obtained using a grating based imaging spectrometer, which creates a spectrogram as a linear function of wavelength. Since the PCGP algorithm requires that there be a one-to-one mapping between the delay axis and frequency axis, we must convert wavelength to frequency and (for broad spectrum in particular) apply the nonlinear conversion factor $\left(I_{\omega}(\omega)=\left.\left(\lambda^{2} / 2 \pi c\right) I_{\lambda}(\lambda)\right|_{\lambda=2 \pi c / \omega}\right.$ to each wavelength/frequency signal value [154]. For a given delay range $\tau=-\Delta \tau / 2: \delta \tau: \Delta \tau / 2$, we must interpolate and crop to corresponding frequency range $\omega=\omega_{o}+(-\pi / \delta \tau: 2 \pi / \Delta \tau: \pi / \delta \tau)$ (where $\omega_{o}$ is the carrier frequency) for the PCGP algorithm to work.

\section{C.4 Error analysis}

Uncertainty in the FROG retrieval algorithm is typically approached using what is known as a 'bootstrapping' method, in the sense that it would appear that you get something for nothing('pulling oneself by one's own bootstraps') [155]. Current techniques approach this problem by randomly only using about $50 \%$ of the data, resampling and running the algorithm many times until sufficient statistics are accumulated to calculate a meaningful mean and standard deviation (used for the error bars) of all the retrieved pulses. Simply setting these values to zero and rerunning the algorithm [48] isn't sufficient since the algorithm still attempts to fit these points: simply ignoring these data points in the intensity constraint 
portion of the algorithm appears to be the only viable approach for calculating a FROG error.

The discrete Fourier transforms needed for the PCGP algorithm, however, require the signal to be zero at the boundary of the outer product otherwise 'ringing' occurs because of boundary artifacts. To minimize this ringing, large grids were used to 'pad' the borders of the data with zeros, making the actual signal a smaller portion of the total trace. As a result, simply using only $\sim 50 \%$ of the trace in the intensity constraint resulted in unrealistic pulse retrievals and large errors. Alternatively, we run the algorithm on multiple occasions with different initial seeds which results in similar, but not identical, reconstructions, all with equally low FROG errors. Given enough reconstructions, careful calculation of the mean and standard deviation of all the traces can indicate the consistency of the reconstruction.

Since the phase retrieval doesn't return an absolute value (i.e. $\phi$ and $\phi+2 \pi$ are equivalent), ambiguities in the phase can develop and lead to large standard deviations in phase. Trivial ambiguities can be removed by subtracting out constant value in phase, but due to the structured nature of the pulse, non-trivial relative phase ambiguities creep up in different parts of the pulse. There doesn't exist a non-trivial method to remove this between different retrievals, making traditional error bar analysis difficult. Taking the derivative of the phase with respect to time, however, removes these ambiguities. This is great news since we are only interested in the instantaneous frequency $\omega_{\text {inst }}=\omega_{o}-d \phi / d t$. 


\section{Appendix D SPECFROG MEASUREMENTS ON OMEGA EP}

This chapter discusses some of the details associated with a long-pulse specular FROG measurement at truly fast-ignition relevant conditions (originally submitted as a research grant proposal).

\section{D.1 Principal objectives of the proposed research}

1. Use novel time-resolved measurements of specularly reflected light to study the temporal evolution of ultra-intense laser plasma interactions (LPI)

(a) (Primary) Study the long-term evolution of hole boring in front of solid targets at truly fast ignition (FI) relevant conditions with the full operational power of OMEGA EP at the Laboratory for Laser Energetics (LLE), $1.5 k J$ in $10 p s$ with a peak focal intensity of $5 \times 10^{19} \mathrm{~W} / \mathrm{cm}^{2}$

(b) (Secondary) Continue analysis of previously obtained data on Titan at the Jupiter Laser Facility

i. Ionization dynamics in LPI with different atomic number interfaces

ii. Pulse splitting phenomenon with ultra-thin (sub-wavelength) targets

2. Benchmark 2D cartesian particle-in-cell (PIC) laser-plasma simulations using the commercially available code LSP [104] with specular reflectivity measurements to gain further physical insight on all these phenomena 


\section{D.2 Background information}

Ultra-intense $\left(>10^{19} \mathrm{~W} / \mathrm{cm}^{2}\right)$ laser-plasma interactions are capable of producing relativistic electron beams with a variety of applications including the fast-ignitor approach to inertial confinement fusion [1], creating ultra-short x-ray sources [3], isochorically heating matter to warm-dense states [2] and accelerating ions via Target Normal Sheath Acceleration (TNSA) [61, 147]. Each application has specific requirements on energy spectrum, angular distribution and conversion efficiency where control of various aspects of these relativistic electron distributions is essential to their effectiveness.

Unfortunatley, there is no way to directly measure the hot-electron source generated in relativistic laser-plasma interaction experiments. However, previous studies of ultraintense laser interactions with extensive under-dense plasma environments have suggested that far-field measurements of the specularly reflected pulse, with the help of particle-in-cell simulations, can provide a great deal of information about the interaction. For example, changes in divergence of the specular beam (relative to the input) from the shape of the relativistic critical surface has been shown to be a strong indicator of the under-dense plasma density profile near the critical surface [78]. Spectral broadening and shifting of the specular pulse via relativistic self-phase modulation have also been found to be sensitive to pre-plasma environment [42] and polarimetry measurements of harmonics generated near the relativistic critical surface have been used to infer self generated megagauss magnetic fields near the interaction surface [82, 149].

Novel, time-resolved measurements of the specularly reflected pulse, made recently by our group on Titan at the Jupiter Laser Facility at Lawrence Livermore National Laboratory, have opened up a new regime for studying laser-plasma interactions with temporal resolution never before seen for this type of experiment. Using a technique known as FROG (frequency resolved optical gating [108]), we have been able to observe differences in dynamic ionization using different $Z$ materials and study pulse splitting phenomenon from sub-wavelength thick $S i_{3} N_{4}$ targets used for proton acceleration (still active research areas I am hoping to continue with support from this proposal). We have also shown how shifts in the instantaneous 
wavelength of the specularly reflected pulse, as measured with a FROG, can be used to study electron density profile steepening [35]. This study revealed that the competition between electron density profile steepening from the laser and heated plasma expansion is not always negligible in this regime $\left(150 \mathrm{~J}, 1.4 \mathrm{ps}, 1 \times 10^{20} \mathrm{~W} / \mathrm{cm}^{2}\right)$. However, the reversion of inward to outward motion of the reflection point of the light occurs near the peak intensity of the 1.4ps laser pulse so that the reversion could be due to either increase in plasma pressure or decrease in laser intensity. Therefore, it remains uncertain that with longer pulses relevant to the full-scale fast ignition, whether the light pressure or the plasma pressure will win the competition on $10 s$ of picoseconds time scale at these intensities. A final answer to this question will critically affect laser absorption [90] and conversion efficiency into hot electrons [27] as well as the required driver energy for fast-ignition. A FROG measurement in this regime will provide a novel insight into the long term behavior of electron density profile steepening and is the primary focus of this proposal.

\section{D.3 Experimental design and approach}

By measuring the instantaneous wavelength shifts of the specularly reflected pulse throughout the interaction with an experiment on OMEGA EP, we hope to understand the dynamics of the laser-plasma interaction at truly fast-ignition relevant scales. This will be done using a polarization gated (PG) FROG, dubbed SPECFROG, and is the primary focus of this proposal.

\section{D.3.1 PG FROG background}

In principle, a PG FROG measurement is an autocorrelation of the pulse that has been resolved in frequency with an imaging spectrometer. The result is a real valued, $2 D$ intensity distribution $I_{P G}$ as a function of relative delay $\tau$ and frequency $\omega$ (also known as a

spectrogram) given by Equation C.1 (where $\widetilde{E}(t)$ is the complex electric field of the pulse). A sample FROG spectrogram from the Titan campaign [35] is shown in Figure D.1(a).

The basic geometry of a PG FROG is shown in Figure C.1. After aligned to the irises, 

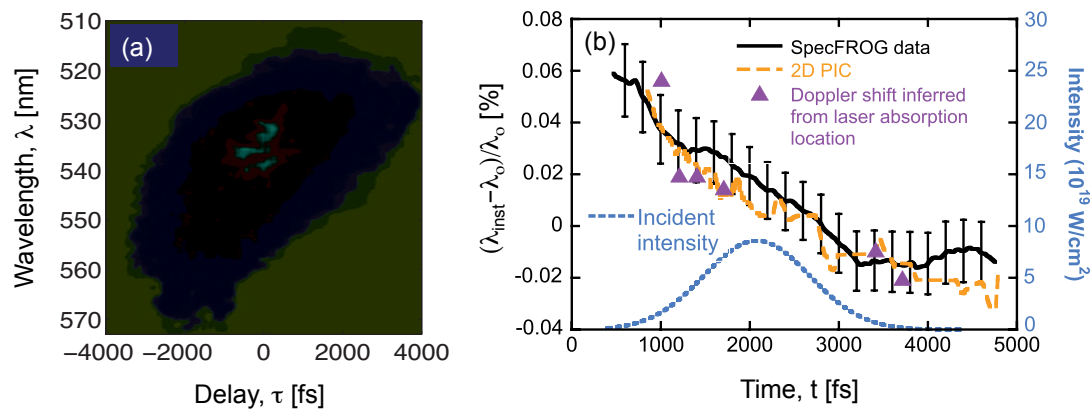

Figure D.1: (Color) (a) Experimental FROG trace obtained on Titan and (b) reconstructed pulse.

the pulse in question is split in two using a beamsplitter (BS): the transmitted pulse (dubbed 'signal') and the reflected pulse (dubbed 'gating'). The polarization of the gating pulse is then rotated 45 deg with respect to the signal pulse (blue) and both are focused down to a line and spatially overlapped on the nonlinear medium (SF5 Schott glass) using cylindrical lenses $\left(L_{1}\right.$ and $\left.L_{2}\right)$. In this single-shot configuration, the two paths cross at an angle such that the spatial dimension can be mapped to a relative delay between the two pulses. The gating pulse induces a nonlinear birefringence in the medium via the optical Kerr effect and an autocorrelation of the signal and gating pulses is obtained (green). The polarization of the autocorrelated pulse is rotated with respect the the signal pulse such that part of it can pass through the analyzer (polarizer orientated 90 deg with respect to the signal pulse polarization) which is used to block the unmixed light. The signal is then imaged and resolved in frequency with an imaging spectrometer.

Using an iterative phase retrieval algorithm, the full temporal pulse information (i.e. instantaneous intensity and phase) can be uniquely retrieved with sub pulse duration resolution using the real valued spectrogram [109]. The instantaneous wavelength, useful for the study of electron density profile steepening, is then simply calculated with a derivative of the phase (shown in black in Figure D.1(b)). Also shown are the full scale $2 D$ PIC simulations done with PSC-HYBRID [148, 156] with pre-plasma environment modeled by HYDRA [157]. The instantaneous wavelength shift $\left(\Delta \lambda / \lambda_{o}=\left(\lambda_{\text {inst }}-\lambda_{o}\right) / \lambda_{o}\right)$ of the specularly reflected 
light from the simulation is shown in yellow and found to be in very good agreement with the data. While there are many ways to get spectral shifts, such as from relativistic phase modulation or ionization, the shifts observed in the experiment have very good agreement with the Doppler shift caused by the motion of the laser absorption point $\left(\Delta \lambda / \lambda_{o}=2 v / c\right.$ here $v$ is the velocity of the reflection point and $c$ is the speed of light).

The PG FROG design is particularly advantageous, as compared to other geometries, as it contains no ambiguity in time directionality and offers very broad bandwidth measurements since phase-matching conditions are automatically satisfied [150]. Given the past success on Titan, we are confident that this approach will produce high quality data on OMEGA EP.

\section{D.3.2 Requirements}

A variety of requirements on the facility, laser, target configuration and diagnostic must be fulfilled for a successful campaign. We will use the OMEGA EP backlighter laser as the main pulse with $1.5 \mathrm{~kJ}$ of energy delivered in a $10 \mathrm{ps}$ pulse duration at the fundamental wavelength $1054 \mathrm{~nm}$. It will be incident on the target (planar aluminum targets, $\sim 1 \times 1 \times$ $0.1 \mathrm{~mm}^{3}$ ) with approximately a $15 \mathrm{deg}$ incident angle. From the facility, we require a fixed port window located along the specular direction. It will have an optical grade window and a mechanical shutter to protect the window when the SPECFROG diagnostic is not in use. We require that the transport optics used to relay and down collimate the specular light will have a pointing stability of less than $\sim 0.2 \mathrm{mrad}$ and deliver a collimated $2 \mathrm{~cm}$ diameter beam at the entrance to the SPECFROG diagnostic (exclusively located outside the target chamber). The SPECFROG will be located on the floor of the target bay, almost directly below the chamber, and secured with kinematic mounts. The ccd camera (Apogee Alta ${ }^{\circledR}$ U8300) and spectrometer (Ocean Optics ${ }^{\circledR}$ U2000) used in the diagnostic require a nearby power strip and BNC trigger $50 \mathrm{~ms}$ in advance of the interaction (TTL, High-Z). The data from these electronics will be sent to an LLE controlled computer with a fiber coupled USB range extender and the acquisition software will be integrated into the LLE systems.

The SPECFROG itself must be able to accommodate the measurement of the $10 \mathrm{ps}$ 
OMEGA EP pulse. It will look not at the fundamental laser wavelength $(1054 \mathrm{~nm})$ : rather, the second harmonic generation created near the critical surface at $527 \mathrm{~nm}$ will be measured. The temporal window of the SPECFROG will be at least $30 \mathrm{ps}$ with better than $500 \mathrm{fs}$ resolution. The spectral window will be at least $100 \mathrm{~nm}(527 \pm 50 \mathrm{~nm})$ with $\sim 0.1 \mathrm{~nm}$ resolution to accommodate up to $10 \%$ shifts in the spectrum. An independent measurement of the spectrum will be obtained using a fiber-coupled spectrometer and will serve as an absolute reference for the phase retrieval algorithm. Shielding from hard hits and electromagnetic interference will also be necessary. The SPEcFROG will contain an LLE approved green diode laser which will be used for internal diagnostic alignment as well as external alignment to the transport optics and target inside the chamber.

\section{D.3.3 Design}

Transport optics will be used to collect, relay, down collimate and direct the specularly reflected light into the SPECFROG. The schematic layout of transport optics is shown in Figure D.2. After passing through a short-wave pass filter (block $1 \omega$, transmit $2 \omega$ ), the specular light will be collected by a $f=1 \mathrm{~m}$, 4" diameter positive lens approximately $2 m$ from the target chamber center (TCC). If second harmonic generation on OMEGA EP experiment scales similarly to previous Trident and Titan results, we will collect approximately $10 \mathrm{~mJ}$ of $2 \omega$ light in an $f / 20$ cone. The light is then collimated using a $f=-50 \mathrm{~cm}$, 2" diameter negative lens (approximately $1.7 \mathrm{~m}$ away from the first positive lens) into the $2 \mathrm{~cm}$ diameter spot size required by the SPECFROG. Because the FROG will be located on the floor below the chamber, several mirrors will be used to direct the light to the FROG entrance irises.

A new FROG had to be designed for the experiment to accommodate the longer pulse durations at OMEGA EP (as our current setup used on Titan are only capable of measuring $\sim$ picosecond pulses). The current SpecFROG model (designed by myself, Yuan Ping and Anthony Link) proposed for the OMEGA EP measurement is shown in Figure D.3. The pulses mix in the SF5 nonlinear medium at an angle of 60 deg (outside the medium), resulting in a theoretical delay window 73.4 ps. The geometric temporal blurring [151] 


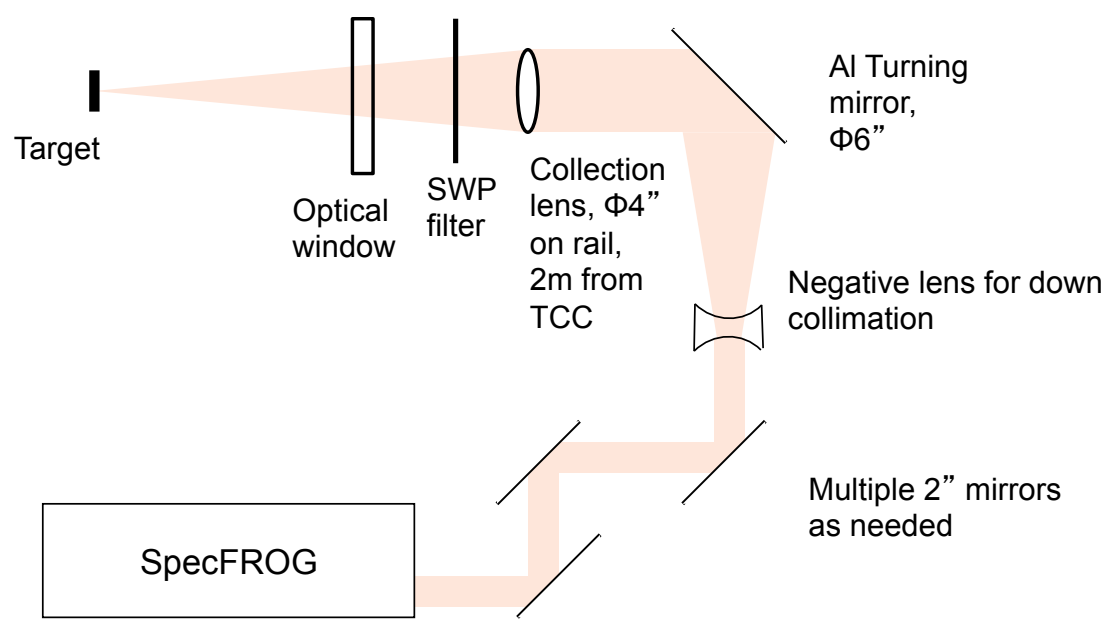

Figure D.2: Schematic of specular pulse transport and down-collimation into the SPECFROG diagnostic.

(likely the limiting effect on temporal resolution at these mixing angles) should be limited to around $0.4 \mathrm{ps}$ so long as the SF5 is no thicker than $500 \mu \mathrm{m}$ thick. Hard hits on the ccd from high energy x-rays produced in the interaction (less than a few hundred $\mathrm{keV}$ ) will be blocked using 1/4" thick tungsten shielding. Alignment protocols, both internal and external to the diagnostic, have already been drafted (although not addressed here).

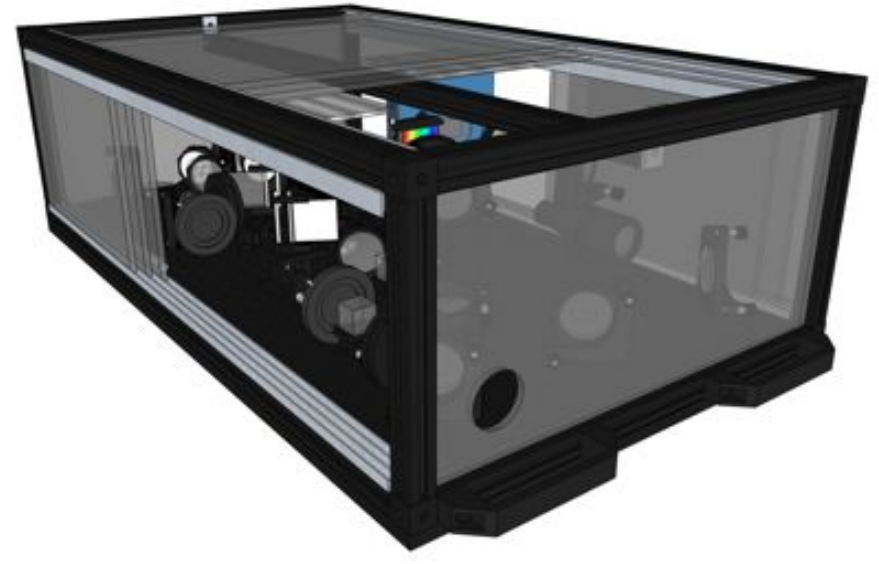

Figure D.3: (Color) Sketch of proposed OMEGA EP SpecFROG by G. Elijah Kemp (2013). 


\section{D.3.4 Campaign, support and simulations}

The proposed experiment, submitted as a Laboratory Basic Science (LBS) experiment in March 2012, has already been accepted by the University of Rochester Laboratory for Laser Energetics for the 2013 Fiscal Year on the OMEGA EP Facility. A total of 5 shots on August 29, 2013 have been awarded (along with two weeks of alignment time on MTW in April 2013 and ride-along opportunities on other experiments on April 30 and May 6, 2013 ). A preliminary design review with LLE has already been completed and the budget for the SPECFROG parts needed for this novel measurement on the OMEGA EP laser has already been approved.

This laboratory basic science experiment directly supports a major program funded by the DOE Office of Fusion Energy Science (OFES) in the fields of relativistic laser-plasma interaction physics and fast ignition: the Fast Ignition High-Energy-Density Science Program (P.I., P. K. Patel) is developing a state-of-art simulation capability for integrated modeling and design of all aspects of a fast ignition target. The simulation tools combine PIC, hybridPIC transport, and radiation-hydrodynamics ICF codes (specifically LsP, PSC- HYBRID, ZUMA and HYDRA). A major requirement of the program is high quality experimental data at OMEGA EP and NIF ARC scale with which to benchmark and validate the codes.

However, I will be graduating shortly (expected April-May 2013). Without support from the FES Postdoctoral Research Program, I will be forced to find employment elsewhere and the SpecFROG project will become dangerously undermanned. Not only would the SPECFROG project benefit from my previous experimental experience with FROG measurements on Titan and Trident, it will also receive continued support in design, experimental data acquisition and analysis. In addition to experimental experience, I also have extensive experience with large scale PIC simulations of laser-plasma interactions with LSP $[70,78,146]$ and could continue to provide simulation support with the help of the the first class computational infrastructure at LLNL. 


\section{D.4 Expected results; significance and/or application of ex- pected results}

Hole boring is the original scheme proposed for fast ignition [1]. The process of hole boring is extremely time-dependent as the plasma density gradient, the temporal pulse shape and the on-target laser intensity, which can be different from vacuum focus intensity due to filamentation, are all varied in time. The complex physics of ultra-intense laser-plasma interaction is sensitive to the detailed balance between these parameters and thus it is essential to perform the fundamental time-resolved measurements we describe on the OMEGA EP laser which is operating in uncharted parameter space of peak laser power, intensity and pulse length. Even for the cone-guided FI scheme, knowing the location of relativistic critical density (and hence the standoff-distance between electron source generation and the fuel core) is also crucial for determining the required FI driver energy since the ignitor energy scales as standoff-distance ${ }^{4}$ [158]. Assuming the same electron divergence, a small $10 \%$ decrease in the distance by hole boring could lead to a $40 \%$ drop in the driver energy, which will be significant for the FI point design. We have already published high quality data on Titan and expect that our results on OMEGA EP will make a major contribution to the fields of fundamental relativistic laser-plasma physics and fast ignition in an experimentally uncharted regime.

\section{D.5 Relevance of proposed research to facilitys programs and goals}

By working jointly with LLE and LLNL, we simultaneously can fulfill aspects of each facilities mission statement. In addition to enhancing the nation's defense and reducing global threats from terrorism and weapons of mass destruction, Lawrence Livermore National Lab-

oratory has a mission to "strengthen the application of world-class science and technology and respond with vision, quality, integrity and technical excellence to scientific issues of national importance." The Laboratory for Laser Energetics at the University of Rochester has a five-fold mission statement: (1) To conduct implosion experiments and basic physics 
experiments in support of the National Inertial Confinement Fusion (ICF) program, (2) To

develop new laser and materials technologies, (3) To provide graduate and undergraduate education in electro-optics, high-power lasers, high-energy-density physics, plasma physics, and nuclear fusion technology, (4) To operate the National Laser Users' Facility and (5) To conduct research and development in advanced technology related to high-energy-density phenomena.

Not only does this project meet every one of these needs, the SPECFROG will be extremely beneficial for all subsequent users of OMEGA EP for the design and interpretation of experiments related to x-ray source generation, high energy density physics, and fast ignition. It will also be invaluable for addressing the fundamental physics of relativistic lasermatter interaction through validation of integrated short-pulse modeling tools including PIC and hybrid-PIC codes.

\section{D.6 Aspects of the facility which provide unique opportuni- ties for conducting the proposed research}

At present, OMEGA EP is the only laser in the world to operate in a regime truly relevant for fast ignition: a laser intensity of $5 \times 10^{19} \mathrm{~W} / \mathrm{cm}^{2}$ in a $10 \mathrm{ps}$ duration pulse. Hence an experimental campaign on EP will provide the unique opportunity of studying the long term dynamics of hole boring and the ensuing effect on hot electron generation. In addition to the FROG technique expertise, LLNL provides the computation resources necessary for the interpretation of the data with large-scale PIC and radiation hydrodynamics codes. 